\title{
Functionalisation of Lead Halide Perovskites with Anthracene
}

By

Jake James Hardy

\begin{abstract}
A Thesis
Submitted to the Victoria University of Wellington

In fulfilment of the requirements for the degree of

Master's by Thesis
\end{abstract}

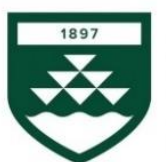

VICTORIA UNIVERSITY OF

WELLINGTON

TE HERENGA WAKA

Victoria University of Wellington

(2020) 


\begin{abstract}
After only a decade's worth of research and development, lead halide perovskites are set to become the basis of a new generation of cheap and highly efficient photovoltaic devices. However, traditional lead halide perovskites such as methylammonium lead triiodide or formamidinium lead triiodide suffer from an intolerance to moisture and high temperatures. 2D Ruddlesden-Popper lead halide perovskites and all inorganic caesium lead halide perovskites have gained attention in recent years due to their improved stability with respect to these environmental conditions.

Like all solar cell technologies, simple lead halide perovskite solar cells have a theoretical maximum efficiency limit of around 30\%. Coupling organic fluorophores to semiconducting nanomaterials is a potential route to circumventing and exceeding this efficiency limit. In this work attempts are made to couple anthracene functional groups to 2D Ruddlesden-Popper lead halides and caesium lead trihalide perovskites. The results of this study show that energy transfer does occur between anthracene and caesium lead trihalide nanocrystals, resulting in increased perovskite emission. These results show that organic fluorophores can be utilised in conjunction with perovskite semiconductors, opening up the possibility of circumventing efficiency limits for perovskite photovoltaic technologies.
\end{abstract}




\section{Acknowledgements}

This work was carried out under the supervision of Dr Nathaniel J. L. K. Davis.

I also want to acknowledge the entire Advanced functional luminescence group for their assistance, guidance, and friendship during the course of this research, as well as the School of Chemical and

Physical Sciences, Victoria University of Wellington, Wellington, New Zealand. 
$\begin{array}{ll}\text { Abstract } & 2\end{array}$

Acknowledgements 3

1. Introduction and Literature Review

- 1a. General Background on Solar Cells and the Shockley-Queisser Limit 7

2. Methylammonium and Formamidinium Lead Trihalide Perovskites 11

- 2a. The Rise of Methylammonium Lead Trihalide Perovskite based Photovoltaics 11 $(1958-2011)$

- 2b. Formamidinium versus Methylammonium Lead Trihalide Perovskites (2013- 15 2015)

- 2c. Addressing Perovskite Thin-film Quality (2015-2020) 16

3. Caesium Lead Trihalide Perovskites 20

4. General Properties and Characteristics of Lead Halide Perovskites 24

- 4a. Optical Absorption Spectrum and Generation of Free Charges 24

- 4b. Defect Tolerance and Rates of Recombination 26

5. Moisture susceptibility and 2D Ruddlesden-Popper Perovskites 28

- 5a. Water Induced Degradation 28

- $\quad$ 5b. Introducing 2D Ruddlesden-Popper Perovskites 29

- 5c. From Three to Two Dimensions, How the Electronic Properties Change 31

- 5d. 2D/3D hybrid Perovskites, The Best of Both Worlds 32

6. Utilising Organic Molecules to Overcome the Shockley-Queisser Limit 35

- 6a. Energy Transfer between Organic Molecules and Inorganic Semiconductors 35

- 6b. Anthracene 39 
- 8a. Synthesis of $N$-(Aminoalkyl)Anthracene-9-Carboxamides

- $\quad 8$ b. Results and Discussion on the Synthesis of $N$-(aminoalkyl)anthracene-9carboxamide

- 8 c. Concluding Remarks on the Synthesis of $N$-(aminoalkylanthracene-9Carboxamides

9. (Anthracene-9-Carboxamido)Alkylaminium Lead Halide ([ACAA] $]_{2} \mathrm{PbX}_{4}$ )

\section{Thin-Films}

- 9a. Synthesis of (ACAA $)_{2} \mathrm{PbX}_{4}$ Thin-Films

- 9b. (ACAA) ${ }_{2} \mathrm{PbX}_{4}$ Thin-Film Results and Discussion

- 9c. Concluding Remarks on (Anthracene-9-Carboxamido)Alkylaminium Lead Halide $\left([\mathrm{ACAA}]_{2} \mathrm{PbX}_{4}\right)$ Thin-Films

10. Intercalation of (Anthracene-9-Carboxamido)Alkylaminium Halides (ACAAX) into Lead Halide Nanocrystals

- $\quad$ 10a. Synthesis of Lead (II) Halide Nanocrystals

- $\quad$ 10b. Results and Discussion of Lead (II) Halide Nanocrystal Synthesis

- $\quad$ 10c. Intercalation of (Anthracene-9-Carboxamido)Alkylaminium Halide Salts into Lead Halide Nanocrystals

- $\quad$ 10d. Intercalation of (Anthracene-9-Carboxamido)Alkylaminium Halide Salts Results and Discussion

- 10e. Concluding Remarks on the Attempts to Intercalate (Anthracene-9-carboxamido) Halide Salts into Lead Halide Nanocrystals 
- 11a. Open-Air Synthesis of $\mathrm{CsPbX}_{3}$ and $\mathrm{CsPbX}_{3}$-AL Nanocrystals 116

- 11b. Results and Discussion of Open-Air Synthesised $\mathrm{CsPbBr}_{3}-\mathrm{AHpAC} \quad 120$

- 11c. Concluding Remarks on Open-Air Synthesised $\mathrm{CsPbBr}_{3}-\mathrm{AHpAC} \quad 131$

12. Coordination of Anthracene Based Ligands to Hot-Injection Synthesised 131 $\mathrm{CsPbX}_{3}$ Nanocrystals

- 12a. Hot-Injection Synthesis of $\mathrm{CsPbX}_{3}$ Nanocrystals 133

- $\quad$ 12b. Results of Hot-Injection Synthesised $\mathrm{CsPbX}_{3}$ and $\mathrm{CsPbX}_{3}$-AL Nanocrystals 137

- 12c. Concluding Remarks on Hot-Injection Synthesised $\mathrm{CsPbX}_{3}$ and $\mathrm{CsPbX}_{3}-\mathrm{AL} 158$ Nanocrystals

13. Concluding Remarks on the Functionalisation of Lead Halide Perovskites 159 with Anthracene

14. References 


\section{Introduction and Literature Review}

\section{1a. General Background on Solar Cells and the Shockley-Queisser Limit}

As the world accelerates its transition away from fossil fuel dependency, maximising the potential of renewable energy sources has become a significant field of research. One of the many alternatives to fossil fuels is solar power which generates electrical energy by either concentrating sunlight to drive a heat engine or by directly converting photons emitted from the sun into electrical current via the photoelectric effect. Solar has significant potential due to the abundance of energy that the sun provides. At the equator on a clear day, every square meter can receive as much as $1361 \mathrm{~W} \mathrm{~m}^{-2} .{ }^{1,2}$ However, while the energy supply is theoretically more than enough to meet the worlds energy demands, harvesting this energy in reality involves overcoming significant obstacles. With regards to the direct conversion of the suns light into electricity, there is a limit on the conversion efficiency of simple photovoltaic devices.

This limit is referred to as the Shockley-Queisser Limit and was first described by William Shockley and Hans J. Queisser in $1961 .{ }^{3}$ Shockley and Queisser determined that for a single p-n junction photovoltaic cell the maximum efficiency of that device can be no more than $33.7 \%$. For clarity, a single p-n junction photovoltaic cell refers to a device composed of two semiconductor crystals. One of the crystals is doped with an electron donating element to provide extra electrons while the other is doped with an electron accepting element to provide electron holes to the crystal. These crystals are referred to as n-type and p-type semiconductors respectively and are placed into contact with each other, forming a p-n junction (see Figure 1a). When in contact, an exchange of additional charges is able to take place between the semiconducting crystals, this is referred to as the drift current. For the n-type semiconductor the loss of electrons and gain of electron holes results in a net positive charge developing near the junction while the loss of electron holes and gain of electrons in the p-type semiconductor produces a negative charge. This region of charge between the two crystals becomes depleted of mobile carriers and is fittingly called the depletion region. The electrical charge of this depletion region produces an electrical field that influences the remaining mobile carriers outside the charged region to retreat away from the junction. This counter flow increases until it becomes equal to the drift current, resulting in an equilibrium of charges travelling to and from the depletion region. At this point, the fermi energy levels of the two crystals are equal, and the overall system is in thermal equilibrium. 


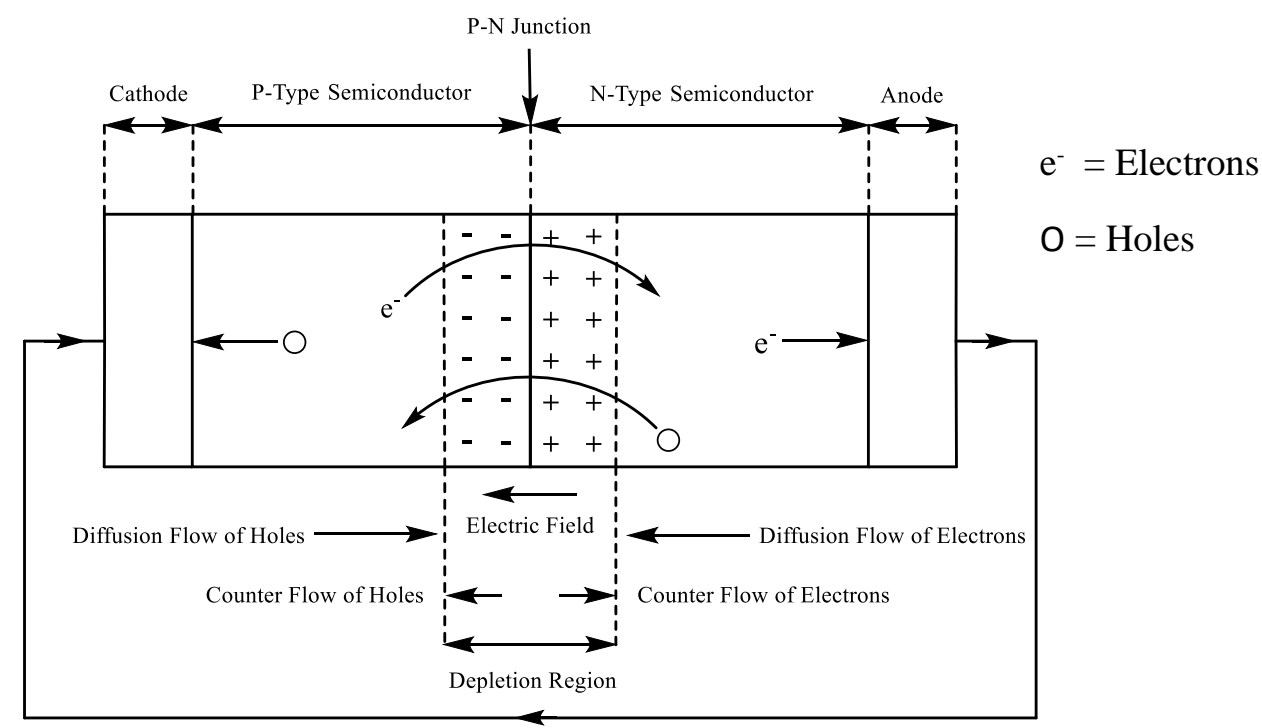

Figure 1a. A simple p-n junction demonstrating the flow of charge carriers within the two semiconductors.

This equilibrium of charge flow acts as an energetic barrier preventing a further net change in charge exchange between the two crystals. When the cell is illuminated with sufficiently high energy light, electrons are promoted into the conduction band of the semiconductor that they were created in and leave a hole in the valence band. If an excitation event occurs within the depletion region, the electric field drives the excited electron into the n-type semiconductor and the hole into the p-type. If an electron hole pair is created within the n-type semiconductor, the excited electron can flow to an electrode and through an external circuit. The hole propagates through the material until it reaches the depletion zone, where the electrical field sweeps it across the junction and into the p-type semiconductor. If an excitation event occurs in the p-type semiconductor, the holes diffuse to the counter electrode where they combine with electrons that have travelled through the external circuit while the excited electrons propagate and cross the junction into the n-type semiconductor.

The low efficiency of such a device becomes apparent when considering several major factors that influences its operation.

1) All objects emit blackbody radiation which is proportional to their temperature. Any inefficiencies in a solar cell will result in heat production, increasing the temperature of the cell and therefore increasing the blackbody radiation. This conversion of absorbed light into radiated heat continues until the temperature of the cell is in equilibrium with its surroundings. Assuming an operating temperature of 300 Kelvin $\left(\sim 26.85^{\circ} \mathrm{C}\right)$, blackbody radiation represents a $7 \%$ decrease in total power conversion efficiency. ${ }^{4}$ 
2) Radiative recombination of an electron/hole pair. Recombination is an inevitable process and ultimately determines the lifetime of photogenerated electrons and holes.

3) Impedance matching. Impedance refers to a systems opposition to the flow of electrical current. When an electrical energy source is delivering power to a circuit (load), the maximum power (measured in Watts) that is possible occurs when the impedance of the energy source (output impedance) matches that of the load (input impedance).

4) Spectrum losses represent the most significant limiting factor on a solar cells efficiency. Sunlight is not composed of a single wavelength of light but is a continuous spectrum of many different wavelengths ranging from $100 \mathrm{~nm}$ in the UV to $1 \mathrm{~mm}$ in the far-infrared. However, a single junction semiconductor will have a single band gap value. Consequently, only one wavelength of the suns light will be optimal for photoexcitation. Any photon with more than the minimum required energy will generate a photoexcitation event but the additional energy will be converted into waste heat. On the other hand, a lower energy photon will not result in a photoexcitation event and is completely useless. Decreasing the band gap will increase the range of the spectrum that can be utilised, but it will also increase the production of waste heat. Conversely, increasing the band gap will reduce waste heat but cut off a greater range of the usable spectrum. As determined by Shockley and Queisser, the optimal band gap that balances these two energy losses has a value of $1.1 \mathrm{eV}$, however, even with such a band gap, the spectrum losses alone reduce the maximum efficiency to $44 \%$.

It is important to note that the Shockley Queisser limit does not consider non-radiative recombination processes nor the mobility of charges through a material. Taking these factors into account further reduces the maximum efficiency.

\begin{tabular}{|c|c|c|c|c|c|c|}
\hline Silicon & Silicon & Silicon & Gallium & Gallium & Cadmium & Perovskite \\
\hline Single & Multi- & Thin-film & Arsenide & Arsenide & Telluride & Cell \\
\hline \multirow[t]{2}{*}{ Crystal } & Crystalline & Crystal & Single & Thin-Film & Thin-Film & \\
\hline & & & Crystal & Crystal & & \\
\hline $26.7 \%$ & $23.3 \%$ & $21.2 \%$ & $27.8 \%$ & $29.1 \%$ & $22.1 \%$ & $25.2 \%$ \\
\hline $\begin{array}{l}\text { Figure } 1 b \\
\text { technologi }\end{array}$ & table of & current & ncy re & for the $m$ & t prominen & photovoltaic \\
\hline
\end{tabular}

Crystallised silicon is by far the most commonly utilised material for photovoltaic devices due to its non-toxicity, ideal band gap of $1.1 \mathrm{eV}$, long term high efficiency, and well developed manufacturing methods. However, crystalline silicon has a theoretical maximum efficiency of $\sim 29 \%$, and the current efficiency record for a silicon based solar cells is $26.7 \% .^{3,5,6-7}$ While there is still room for improvement, 
new technologies are currently in development that hold the promise of greater efficiencies for lower production costs (See Figure $\mathbf{1 b}$ ). One of these emerging technologies are perovskite based solar cells. 


\section{Methylammonium and Formamidinium Lead Trihalide Perovskites}

\section{2a. The Rise of Methylammonium Lead Trihalide Perovskite based Photovoltaics (1958-} 2011)

Perovskites are a class of materials defined by their characteristic crystal structure. The basic perovskite chemical formula can be generalised as $\mathrm{ABX}_{3}$, with a cubic crystal structure consisting of the large $\mathrm{A}$ cation of $n+$ charge in the centre of the unit cell, the smaller B cation of $2 n+$ charge located at each vertex of the unit cell, and the $\mathrm{X}$ anions of $n$ - charge at the centre of each edge of the cell, see Figure $2 a$.
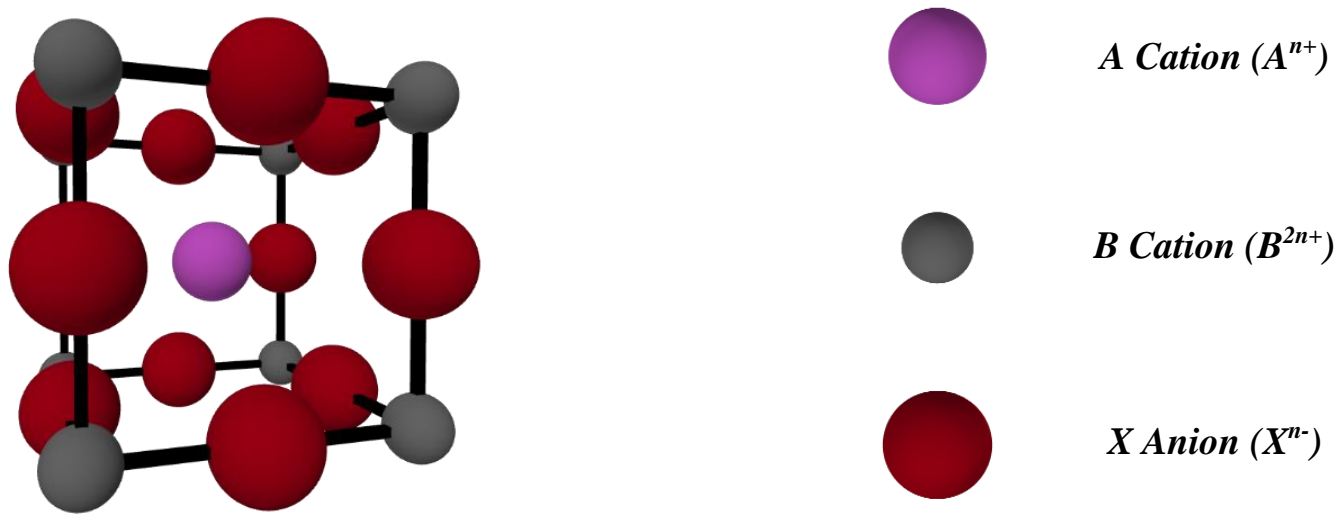

B Cation $\left(B^{2 n+}\right)$

$X$ Anion $\left(X^{n-}\right)$

Figure 2a. The Perovskite Unit Cell, consisting of a lattice of $X$ anions, with $B$ cations occupying the octahedral sites and a central A cation.

Most compounds that possess the perovskite structure are oxides. In fact, the parent compound of this class of materials is calcium titanium oxide $\left(\mathrm{CaTiO}_{3}\right)$ (mineral name is 'Perovskite'). However, 1958 saw the discovery that caesium plumbohalides $\left(\mathrm{CsPbX}_{3}\right)$ also have the perovskite structure. ${ }^{8}$ In these compounds, caesium $\left(\mathrm{Cs}^{+}\right)$is the A cation, lead $\left(\mathrm{Pb}^{2+}\right)$ is the $\mathrm{B}$ cation, and $\mathrm{X}$ can be chloride $\left(\mathrm{Cl}^{-}\right)$, bromide $\left(\mathrm{Br}^{-}\right)$, or iodide $\left(\mathrm{I}^{-}\right)$anions. Significant attention has been drawn to lead halide perovskites since Miyasaka et al (2009) published their investigations into the use of a similar compound, the organicinorganic perovskite methylammonium lead tribromide $\left(\mathrm{CH}_{3} \mathrm{NH}_{3} \mathrm{PbBr}_{3}\right.$ or $\left.\mathrm{MAPbBr}_{3}\right)$ as a sensitizer for mesoporous (mp) titanium dioxide $\left(\mathrm{TiO}_{2}\right)$ in photovoltaic devices. ${ }^{9}$ For $\mathrm{MAPbBr}_{3}$, the methylammonium $\left(\mathrm{MA}^{+}\right)$ion replaces caesium as the A cation. The overall design of the device was based upon the dye-sensitized solar cell (DSSC) architecture (see Figure $2 \boldsymbol{b}$ ). In this example, the (mp) $\mathrm{TiO}_{2}$ was coated with the perovskite film. On top of the coated $\mathrm{TiO}_{2}$ is a transparent electrode composed of fluorine-doped tin oxide (FTO), while underneath is a redox electrolyte solution of lithium iodide/iodine and a platinum $(\mathrm{Pt})$ counter electrode $\left(\mathrm{FTO} /(\mathrm{mp}) \mathrm{TiO}_{2} / \mathrm{MAPbBr}_{3} / \mathrm{LiI} / \mathrm{I}_{2} / \mathrm{Pt}\right)$. Despite this early device demonstrating a low efficiency of only $3.8 \%$ and a stability of only a few minutes, the 
optoelectronic properties displayed by the perovskite sensitizer garnered enough interest to further research and development. ${ }^{9}$

It would not be until 2011 that the first major breakthroughs in perovskite based photovoltaic devices would be made. Park et al (2011) developed a similar solar cell to that developed by Miyasaka's group, but instead utilised methylammonium lead triiodide $\left.\left(\mathrm{CH}_{3} \mathrm{NH}_{3} \mathrm{PbI}_{3} \text { or } \mathrm{MAPbI}\right)_{3}\right)$ as their sensitizer as opposed to $\mathrm{MAPbBr}_{3} .{ }^{10}$ Despite the similarity, this device obtained a maximum power conversion efficiency of $6.5 \%$, almost doubling the previous record. Such an improvement can be attributed to the more ideal band gap of $1.55 \mathrm{eV}$ for $\mathrm{MAPbI}_{3}$, as opposed to $2.3 \mathrm{eV}$ for $\mathrm{MAPbBr}_{3}$, reducing spectrum losses. ${ }^{10}$

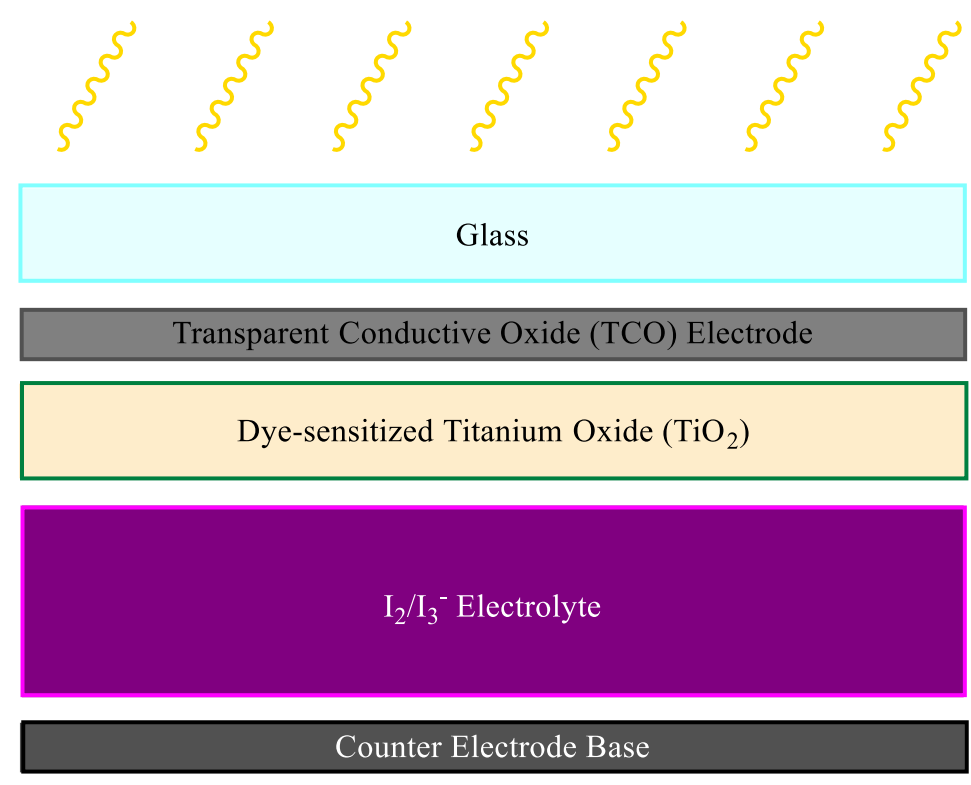

Figure 2b. The essential components of a dye-sensitised solar cell (DSSC).

The change in band gap between $\mathrm{MAPbBr}_{3}$ and $\mathrm{MAPbI}_{3}$ is a direct result of the substitution of bromine for iodine and is a demonstration of how the elemental composition of the material influences the electronic properties and allows for fine tuning. For methylammonium lead trihalide perovskites, the band gap can range from a minimum value of $1.55 \mathrm{eV}$ for $\mathrm{MAPbI}_{3}$ all the way to nearly $3.0 \mathrm{eV}$ for $\mathrm{MAPbCl}_{3}{ }^{11}{ }^{11}{ }^{12}$ This trend arises from the specific combination of orbitals that contribute to the valence and conduction bands. The valence band is composed primarily of in-plane halogen p orbitals that hybridise out of phase with the lead 6 s orbitals. The degree of orbital overlap directly influences the stability and therefore energy of the valence band maximum. Specifically, chloride based perovskites possess low energy valence band maximums as the small ionic radius of the chloride anion results in minimal orbital overlap and therefore a relatively small destabilising effect. For iodide based perovskites, the large $5 \mathrm{p}$ orbital significantly overlaps with the lead 6 s orbital, resulting in a high energy 
valence band maximum. As a result, the valence band maximum is very sensitive with regards to the halogen composition. ${ }^{13,14}$ On the other hand, the conduction band is composed solely of the unoccupied lead $6 \mathrm{p}$ orbitals and therefore is relatively unaffected by the present halogens. Therefore, altering the halogen composition directly affects the band gap by altering the valence band maximum and leaving the conduction band unaffected.

The year 2012 saw Lee et al (2012) publish the results of their investigations into solution processable solar cells. ${ }^{15}$ While the previously discussed devices utilised the common DSSC architecture, the electrolyte of those devices significantly contributed to the rapid degradation of the perovskite film. To overcome this, this study utilised a different solar cell architecture that eliminates the need of an electrolyte, the p-n junction solar cell. Solar cells of this design require a light absorbing material, an electron transporting n-type semiconductor, a hole transporting p-type semiconductor, and electrodes to which a circuit can be connected. In their study, Lee et al (2009) utilised the perovskite methylammonium lead diiodide monochloride $\left(\mathrm{MAPbI}_{2} \mathrm{Cl}\right)$ as the light absorbing sensitizer for mesoporous (mp) $\mathrm{TiO}_{2}$, which would act as the n-type semiconductor. Above the sensitised $\mathrm{TiO}_{2}$ was a transparent conductive glass (TCO) electrode composed of fluorine-doped tin oxide (FTO) and underneath was the hole transporting material 2,2',7,7'-tetrakis-( $N, N$-di- $p$-methoxyphenylamine) 9,9'spirobifluorene (spiro-OMeTAD or spiro-MeOTAD) as the p-type conductor with a silver (Ag) counter electrode $\left(\mathrm{FTO} /(\mathrm{mp}) \mathrm{TiO}_{2} / \mathrm{MAPbI}_{2} \mathrm{Cl} /\right.$ spiro-OMeTAD/Ag) (see Figure $2 \boldsymbol{c}$ ). The boundary between the $\mathrm{TiO}_{2}$ and spiro-OMeTAD acts as the p-n junction of the device. In addition to this architecture, the group also produced a second device which replaced the mesoporous $\mathrm{TiO}_{2}$ with non-conductive $\mathrm{Al}_{2} \mathrm{O}_{3}$, but was otherwise identical. The $\mathrm{TiO}_{2}$ based device was not only more stable than previously reported devices, but also exhibited a power conversion efficiency of $8 \%$, a significant improvement in of itself. However, the more interesting result came from the alumina based device which demonstrated a power conversion efficiency of $10.9 \%$, a faster rate of charge transport, and an open circuit voltage of $1.1 \mathrm{~V}$, more than 200 millivolts greater than the $\mathrm{TiO}_{2}$ device. ${ }^{15}$ 


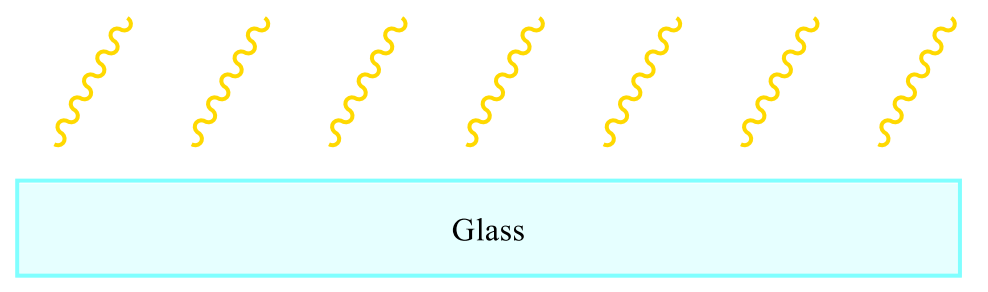

Flourine-doped Tin Oxide (FTO)

$\mathrm{MAPbI}_{2} \mathrm{Cl}$ Sensitised Titanium Oxide $\left(\mathrm{TiO}_{2}\right)$

Spiro-OMeTAD

Silver (Ag) Counter Electrode

Figure 2c. Diagram of the p-n junction solar cell constructed by Lee et al (2012).

The authors attributed the faster rate of charge transport and increased open-circuit voltage to the perovskite sensitizer. In the standard $\mathrm{TiO}_{2}$ device the perovskite acts simply to absorb light and generate free charges. Excited electrons are then transferred to the $\mathrm{TiO}_{2}$ layer where they can be transported to the circuit while the holes travel to and through the spiro-OMeTAD. However, replacing the $\mathrm{TiO}_{2}$ with insulating $\mathrm{Al}_{2} \mathrm{O}_{3}$ confines the excited electrons to the perovskite. Therefore, it must be concluded that the observed increase in charge transportation rate is a feature of the $\mathrm{CH}_{3} \mathrm{NH}_{3} \mathrm{PbI}_{2} \mathrm{Cl}$ sensitizer. A faster rate of charge transport reduces the window of time that processes such as radiative and non-radiative recombination can occur before charge carriers exit the cell, reducing the negative effect on the power conversion efficiency. With regards to the open circuit voltage $\left(V_{O C}\right)$, which is the difference in electrical potential between the two electrodes of the device at zero current, the authors explain that the perovskite must possess fewer surface and sub-band gap states than mesoporous $\mathrm{TiO}_{2}$ and therefore has less capacity per unit volume for storing charge carriers. In other words, the perovskite has a lower chemical capacitance. As Bisquert (2003) found, a lower chemical capacitance raises the quasi-fermi level of the electrons with respect to the conduction band which in turn raises the voltage. ${ }^{16}$ Therefore, confining the electric charges to the perovskite results in a greater splitting of the fermi-levels between the n-type and p-type semiconductors and therefore a higher $V_{O C}$. Results such as this demonstrated that lead trihalide perovskites were more than just another potential sensitizer, and that they possess many remarkable properties that if properly tuned and utilised could result in highly efficient photovoltaic devices. 


\section{2b. Formamidinium versus Methylammonium Lead Trihalide Perovskites (2013-2015)}

The next notable increase in efficiency occurred in 2013 when Noh et al (2013) reported the development of a hybrid heterojunction solar cell with an efficiency of $12.3 \% .{ }^{17}$ The device was of the p-n junction architecture and consisted of a FTO electrode, under which was methylammonium lead tribromide/iodide $\quad\left(\mathrm{MAPb}\left(\mathrm{Br}_{\mathrm{x}} \mathrm{I}_{1-\mathrm{x}}\right)_{3}\right) \quad$ sensitised $\quad(\mathrm{mp}) \quad \mathrm{TiO}_{2}, \quad$ poly[bis(4-phenyl)(2,4,6trimethylphenyl)amine] (PTAA) as a hole transporting material doped with Libis(trifluoromethanesulfonyl)imide (Li-TFSI)/acetonitrile and 4-tert-butylpyridine (tBP), and a gold (Au) counter electrode base (FTO/(mp)TiO $/ \mathrm{MAPb}\left(\mathrm{Br}_{\mathrm{x}} \mathrm{I}_{1-\mathrm{x}}\right)_{3} / \mathrm{PTAA}$ - Li-TFSI - tBP/Au).

The authors explained that the increased performance of this device was due to the tuning of the perovskite composition by substituting iodine with bromine, with the degree of substitution ranging from $0 \%$ to $25 \%$ bromine. Beyond $25 \%$ bromine the gain in performance becomes overshadowed by the increasing band and spectral losses. Within this range it was shown that as the proportion of bromine increased from 0 to $25 \%$, the current density decreased from $18 \mathrm{~mA} \mathrm{~cm}^{-2}$ to $5 \mathrm{~mA} \mathrm{~cm}^{-2}$ while the opencircuit voltage increased from 0.87 to $1.13 \mathrm{~V}$ along with the fill factor $(F F)$ from 0.66 to $0.74 \%$. The fill factor $(F F)$ refers to the quality of a solar cell by comparing the power that is achieved at the maximum power point for the device and the theoretical value based upon the open circuit voltage and short circuit current. All of these observations were attributed to the increasing band gap that results from the substitution of iodine for bromine. Within the ranges of 0 to $25 \%$ bromine, all devices produced an efficiency of over $10 \% .^{17}$

2014 saw Yang et al (2014) surpassing the previous record by optimising the carrier transport pathways found within perovskite based solar cells. ${ }^{18}$ In their work, the authors fabricated solar cells based upon the commonly used p-n junction architecture of doped $\mathrm{SnO}_{2}$, $\mathrm{TiO}_{2}$ with $\mathrm{CH}_{3} \mathrm{NH}_{3} \mathrm{~Pb}\left(\mathrm{I}_{0.25} \mathrm{Cl}_{0.75}\right)_{3}$, spiroOMeTAD, and $\mathrm{Au}$, however, with several important modifications. Firstly, the tin oxide substrate was not the commonly used fluorine doped tin oxide (FTO) but instead was indium doped tin oxide (ITO) and had been additionally modified with a polymer containing aliphatic amines to reduce the work function of the substrate from $4.6 \mathrm{eV}$ to $4.0 \mathrm{eV}$. This increased the electron affinity of the substrate and facilitated the transport of electrons from the perovskite coated $\mathrm{TiO}_{2}$. Secondly, the mesoporous $\mathrm{TiO}_{2}$ was doped with yttrium as it has been demonstrated that the addition of yttrium reduces the recombination rate within the semiconductor. These modifications favourably adjusted the carrier pathway of electrons and culminated in a device with a power conversion efficiency of $19.3 \% .^{18}$

The vast majority of publications up until this point had focused on the methylammonium lead halide perovskites, specifically methylammonium lead triiodide due to its low band gap of $1.55 \mathrm{eV}$. Previously discussed advancements in solar cell performance were achieved via a combination of altering the halogen composition in an attempt to achieve more ideal band gaps and open circuit voltages, or looking 
into optimised solar cell design and construction. However, Eperon et al (2014) published their investigations into substituting methylammonium as the A cation within the crystal structure. It had been proposed that the A cation has little to do with the determination of the band gap and is simply required to neutralise and therefore stabilise the overall perovskite structure. However, this investigation determined that this is not the case. ${ }^{19}$

Eperon et al (2014) synthesised the perovskites caesium lead triiodide $\left(\mathrm{CsPbI}_{3}\right)$ and formamidinium lead triiodide $\left(\mathrm{HC}\left(\mathrm{NH}_{2}\right)_{2} \mathrm{PbI}_{3}\right.$ or $\left.\mathrm{FAPbI}_{3}\right) .{ }^{19}$ Though both of these materials had been grown in their crystalline form this was, as far as the authors were aware of at the time, the first example of a spincoat synthesis for these materials. Interestingly, the results showed that the slightly larger formamidinium produced a series of band gap values between $1.48 \mathrm{eV}$ for $\mathrm{FAPbI}_{3}$ and $2.23 \mathrm{eV}$ and $\mathrm{FAPbBr}_{3}$. The substitution of methylammonium with formamidinium brings the band gap closer to the ideal $1.1 \mathrm{eV}$ and therefore would allow for a greater range of the solar spectrum to be effectively utilised. As such, the authors constructed a p-n junction cell consisting of (in descending order); FTO, mesoporous $\mathrm{TiO}_{2}, \mathrm{FAPbI}_{3}$, spiro-OMeTAD and finally an Au counter electrode. As expected, the best performing device demonstrated an improved efficiency of $14.2 \%$ which was the highest reported value at the time of publication. Besides the benefit of the lower energy band gap and therefore reduced spectrum loss, the efficiency also benefited from the higher short circuit values. ${ }^{19}$

It did not take long for subsequent studies to increase the efficiencies of formamidinium lead trihalide based solar devices. By the end of 2013 such devices had achieved power conversion efficiencies of over $16 \% .{ }^{20}$ However, one major drawback to formamidinium based perovskites is the greater difficulty involved in their stabilisation compared to methylammonium based perovskites. ${ }^{21}$ The increased size of the formamidinium cation results in a destabilisation of the perovskite crystal structure, facilitating thermal and UV induced degradation. Therefore, research on both perovskite materials has continued to move forward due to their respective strengths and weaknesses.

\section{2c. Addressing Perovskite Thin-film Quality (2015-2020)}

One of the first devices to achieve a power conversion efficiency beyond $20 \%$ was a formamidinium lead triiodide based device made in 2015 by Yang et al (2015). ${ }^{21}$ Their approach focused on addressing the common crystallographic defects that arise from commonly used synthetic methods such as sequential deposition, vapor-assisted deposition, solvent engineering, and additive assisted deposition. To achieve improved film quality, the researchers developed an intramolecular exchange process to synthesis the perovskite film.

While perovskites do display a remarkable ability to retain their electronic properties despite the significant presence of crystallographic defects, they are not immune. Common defects that are 
encountered when synthesising perovskite films are the incomplete conversion of the precursor reagents into the perovskite phase, missing ions, and the exchange of $\mathrm{A}$ and $\mathrm{B}$ cations in the crystal structure. Specifically addressing the first issue of incomplete conversion of reagents, the authors employed the use of a source of lead iodide possessing intercalated dimethyl sulfoxide molecules ( $\mathrm{PbI}_{2}(\mathrm{DMSO})$ ). Such a reagent had previously been shown to be effectively converted into the $\mathrm{MAPbI}_{3}$ perovskite via dissolving the $\mathrm{PbI}_{2}$ (DMSO) in $\gamma$-butyrolactone with methylammonium iodide (MAI), an example of the solvent engineering process. ${ }^{22}$ Using a similar method to synthesis the formamidinium equivalent, however, does not produce the same high quality results. Instead the film that is formed possesses morphological features such pin-holes and a rough surface that hinder charge transport and charge transfer. Instead of a solvent engineering process, Yang et al (2015) took advantage of the easily displaced DMSO molecules and directly spin-coated the precursor $\mathrm{PbI}_{2}(\mathrm{DMSO})$ onto their substrate and annealed it to form a high quality thin-film of $\mathrm{PbI}_{2}$ (DMSO). ${ }^{21}$ Afterwards, a solution of formamidinium iodide (FAI) and methylammonium bromide (MABr) in isopropyl alcohol (IPA) was deposited onto the lead iodide film. The intercalated DMSO molecules were easily displaced by the organic salts which have a higher affinity for $\mathrm{PbI}_{2}$ due to their ionic character versus the purely van der Waals interactions of neutral DMSO. This allowed for the effective conversion of the $\mathrm{PbI}_{2}(\mathrm{DMSO})$ precursor into the perovskite phase. In addition, the incorporation of $\mathrm{MABr}$ results in a synergistic effect that stabilises the perovskite phase, a phenomenon that the authors demonstrated in one of their previous publications. ${ }^{23}$ This synergistic effect grants the resulting hybrid perovskite the more ideal band gap of $\mathrm{FAPbI}_{3}$ and the improved stability of $\mathrm{MAPbBr}_{3}$.

The Yang et al (2015) utilised their new method to construct two devices. ${ }^{21}$ The first device consisted of the standard p-n junction architecture consisting of $\mathrm{FTO} /(\mathrm{mp}) \mathrm{TiO}_{2} / \mathrm{FAPbI}_{2} / \mathrm{MAPbI}_{2} \mathrm{Br} / \mathrm{PTAA}$ - LiTFSI - tBP/Au. The best performing device contained $15 \%$ methylammonium bromide by weight and produced a power conversion efficiency of $19.2 \%$. The second device was based upon a n-i-p junction architecture, consisting of a FTO anode, p-type semiconducting nickel oxide (NiO), pure $\mathrm{FAPbI}_{3}, \mathrm{n}$ type phenyl- $\mathrm{C}_{61}$-butyric acid methyl ester $(\mathrm{PCBM})$, lithium fluoride ( $\left.\mathrm{LiF}\right)$, and an aluminium ( $\mathrm{Al}$ ) counter electrode $\left(\mathrm{FTO} / \mathrm{NiO} / \mathrm{FAPBI}_{3} / \mathrm{PCBM} / \mathrm{LiF} / \mathrm{Al}\right)$. This device reached a power conversion efficiency of $20.2 \%$. However, this observation only occurred when then voltage across the device was swept from the open-circuit voltage $\left(\mathrm{V}_{\mathrm{oc}}\right)$ down to zero voltage $\left(\mathrm{V}_{0}\right)$. In the reverse direction, the power conversion efficiency was lower. This phenomenon is referred to as current-density-voltage $(J-V)$ hysteresis and represents a currently unresolved problem in photovoltaics. However, despite this, this publication does represent a major step forward in terms of power conversion efficiency and perovskite thin-film synthesis.

The next significant increase in power conversion efficiency for perovskite solar cells occurred in 2017. Cho et al (2017) sought to decrease the rate of charge recombination at the heterojunction between the 
perovskite and the mesoporous titanium oxide, a significant source of performance loss for solar cells, by passivating the surface of the perovskite film and thereby reduce detrimental morphological features. ${ }^{24}$ Such a technique that has been successfully utilised in various solar cell technologies to increase their performance. The authors synthesised the perovskite $\left(\left(\mathrm{CH}_{2}\left(\mathrm{NH}_{2}\right)_{2} \mathrm{PbI}_{3}\right)_{0.85}\left(\mathrm{CH}_{3} \mathrm{NH}_{3} \mathrm{PbBr}_{3}\right)_{0.15}\right.$ with an excess of lead (II) iodide $\left(\mathrm{PbI}_{2}\right)$. They utilised this excess $\mathrm{PbI}_{2}$ to form a second, bromine enriched perovskite that would share the heterojunction with the $\mathrm{TiO}_{2}$. The authors state that the wider band gap of this secondary perovskite acts as a barrier for charge recombination at the heterojunction and therefore improved the open-circuit voltage of the overall device. ${ }^{24}$

Improving the quality of synthesised perovskite films led to another efficiency increase in March 2016, with the results published in June 2017. As mentioned previously, a common factor that hinders the quality of perovskite thin-films are defects within the crystal structure of the film. Low crystallinity hinders the rate of exciton dissociation, charge transport, and charge diffusion, all of which results in an increase in the recombination rate. One cause for such defects is halide deficiency. In the previously mentioned solvent engineering process developed by Yang et al (2015), the iodine deficient $\left[\left(\mathrm{Pb}_{3} \mathrm{I}_{8}\right)_{n}\right]^{2 n-}$ anionic complex can form and inevitably produce defects within the perovskite film. ${ }^{22}$ To address this detrimental effect, the same team modified the two step intramolecular exchange process by adding additional elemental iodine into the isopropanol (IPA) solution containing the organic salts Yang et al (2017). The iodine oxidises the secondary alcohol, producing acetone and two equivalents of hydrogen iodide. The hydrogen iodide further reacts with remaining iodine to produce the triiodide anion $\left(\mathrm{I}_{3}^{-}\right)$ (see Figure $2 c)^{25}$

$$
\begin{gathered}
\mathrm{CH}_{3} \mathrm{CH}(\mathrm{OH}) \mathrm{CH}_{3}+\mathrm{I}_{2} \rightarrow \mathrm{CH}_{3} \mathrm{C}(\mathrm{O}) \mathrm{CH}_{3}+2 \mathrm{HI} \\
\mathrm{HI}+\mathrm{I}_{2} \rightleftharpoons \mathrm{HI}_{3}
\end{gathered}
$$

\section{Figure 2c. The two step triiodide formation reaction between elemental iodine and acetone.}

Initially an equilibrium is formed, but after seven days of stirring all elemental iodine is converted into $\mathrm{I}_{3}$. The organic salts are then dissolved within an IPA solution containing 1 to $5 \mathrm{mmol}$ of dissolved $\mathrm{I}_{3}$. The solution is then deposited onto the $\mathrm{PbI}_{2}$ (DMSO) substrate to produce the final perovskite layer as in the original method. The authors constructed a series of devices containing a perovskite film synthesised via the modified synthetic method with varying amounts of $\mathrm{I}_{3}^{-}$, and a control device containing perovskite synthesised via the unmodified method. The results showed a positive correlation between increased solar cell performance and the amount $\mathrm{I}_{3}{ }^{-}$with a peak performance correlated with 3 mmol of $\mathrm{I}_{3}^{-}$. Their best device displayed an open-circuit voltage $\left(V_{o c}\right)$ of $1.1 \mathrm{~V}$, a short-circuit current $\left(J_{s c}\right)$ of $24.1 \mathrm{~mA} \mathrm{~cm}^{-2}$, fill factor $(F F)$ of $81.9 \%$, and power conversion efficiency (PCE) of $21.6 \%$, compared to the control device's $V_{o c}$ of $1.07 \mathrm{~V}, J_{s c}$ of $23.5 \mathrm{~mA} \mathrm{~cm}{ }^{-2}$, a $F F$ of $80.8 \%$, and a PCE of 
20.3\%. The authors argue that the increase in the short-circuit current must be a result of an improved light-harvesting capability of the film that results from the improved quality. The improvement in $V_{O C}$ and $F F$ are a consequence of the decrease in crystal defects that facilitate nonradiative recombination, specifically trap-assisted recombination. Further optimising their fabrication procedure resulted in a device with a PCE of $22.1 \%$, with no appreciable hysteresis. ${ }^{22}$

At the time of writing, January 2020, the current world record for a single junction solar cell, according to the national renewable energy laboratory (NREL) is 23.3\% According to the same resource, in 2018 the Oxford University spin-off company Oxford Photovoltaics announced a perovskite/silicon tandem solar cell with a certified power conversion efficiency of $28.0 \%$. Such an efficiency exceeds that of both perovskite and silicon single junction solar devices. As such, within the span of a decade, the power conversion efficiencies of perovskite based solar cells have increased from $3.8 \%$ to $23.0 \%$ in a single junction device and to $28.0 \%$ for a tandem device incorporating silicon. Such a dramatic rate of refinement, coupled with the abundancy of the raw materials, namely hydrogen, carbon, nitrogen, lead, bromine, and iodine, and the solution based synthetic methods lends credibility to their potential as alternatives/replacements for existing photovoltaic devices. 


\section{Caesium Lead Trihalide Perovskites}

The significant rise of organic-inorganic perovskite solar cells has since their inception been hampered by the susceptibility of these materials to moisture, high temperatures, and UV light. This arises due to the relatively weak interactions that hold the organic A cations to the inorganic components of the crystal lattice, allowing for the facile destabilisation of the perovskite phase. Inspired by the stability of purely inorganic semiconducting materials such as $\mathrm{GaAs}, \mathrm{Cu}\left(\mathrm{In}_{1-\mathrm{x}}\right) \mathrm{Ga}_{x} \mathrm{Se}_{2}$, and $\mathrm{CdTe}$, researchers have turned to inorganic monovalent cations such as $\mathrm{Cs}^{+}$and even $\mathrm{Rb}^{+}$to address these stability issues. Studies have shown that doping organic/inorganic perovskites with caesium improves the thermal stability of both methylammonium and formamidinium based perovskites. ${ }^{26,27,28,29}$ Such results into caesium doped perovskites have led to investigations into purely inorganic caesium lead halide perovskites and their potential in photovoltaic devices.

In 2015, Kulbak et al (2015) sought to determine whether or not an organic A cation is essential for photovoltaic applications by measuring and comparing the electronic properties of the purely inorganic perovskite $\mathrm{CsPbBr}_{3}$ to the organic-inorganic $\mathrm{MAPbBr}_{3}{ }^{30}{ }^{3} \mathrm{Via}$ the use of transmission measurements of $\mathrm{CsPbr}_{3}$ on mesoporous titanium oxide $\left(\mathrm{TiO}_{2}\right)$ and fluorine doped tin oxide (FTO) and appropriate analysis of Tauc Plots, the authors approximated the band gap of $\mathrm{CsPbr}_{3}$ to be identical to that of $\mathrm{MAPbBr}_{3}$ at $2.32 \mathrm{eV}$. In addition, ultraviolet photoelectron spectroscopy (UPS) showed that the valence band maximum correlated extremely well with $\mathrm{MAPbBr}_{3}$ at $5.97 \mathrm{eV}$ versus $5.9 \mathrm{eV}$. Constructing several devices based upon the p-n junction architecture; FTO/(mp)TiO $/$ Perovskite/HTM/Au (the authors constructed multiple devices utilising different hole transporting materials (HTM)). The authors report that devices that utilise all inorganic $\mathrm{CsPbBr}_{3}$ perovskites work just as effectively as $\mathrm{MAPbBr}_{3}$ perovskite devices. The best device utilised poly[bis(4-phenyl)(2,4,6-trimethylphenyl)amine] (PTAA) as the HTM and displayed a power conversion efficiency of 5.92\% and 5.72\% on forward and reverse scan respectively. ${ }^{30}$ In addition, Protesescu et al (2015) estimated the excitonic binding energies of $\mathrm{CsPbCl}_{3}, \mathrm{CsPbrr}_{3}$, and $\mathrm{CsPbI}_{3}$ nanocrystals and found these values to be comparable to the equivalent methylammonium based perovskites. ${ }^{31}$ Studies such as these show that the organic component of a lead trihalide perovskite is not necessary for the desired electronic properties of a perovskite to arise, justifying further research in purely inorganic perovskite solar cell research.

The following year, 2016, saw efficiencies rise to $6.2 \%$ for $\mathrm{CsPbBr}_{3}$ based solar cells. ${ }^{32}$ The study behind this development also compared the thermal tolerance of $\mathrm{CsPbr}_{3}$ to $\mathrm{MAPbBr}_{3}$ and showed that while the later will start to degrade at a relatively low temperature of $\sim 220^{\circ} \mathrm{C}$, the inorganic perovskite would remain stable up to $\sim 580^{\circ} \mathrm{C}$. However, like their organic counterparts, the ideal caesium lead halide perovskite for solar cell applications would not be caesium lead tribromide but caesium lead 
triiodide $\left(\mathrm{CsPb}_{3}\right)$. While studies have shown that the thermal stability of $\mathrm{CsPbI}_{3}$ is superior to the equivalent organic-inorganic perovskite, $\mathrm{CsPbI}_{3}$ has its own unique stability issue.

$\mathrm{CsPbI}_{3}$ can possess two crystal structures, a non-perovskite orthorhombic phase $\left(\delta-\mathrm{CsPb}_{3}\right)$ and the traditional perovskite structure, referred to as the cubic phase ( $\left.\alpha-\mathrm{CsPb}_{3}\right)$. While $\alpha-\mathrm{CsPbI}_{3}$ displays all of the desired electronic properties of a lead trihalide perovskite, it requires temperatures above $320^{\circ} \mathrm{C}$ to form and will readily degrade into the orthorhombic phase once cooled to below this formation temperature. Constructing solar devices using $\delta-\mathrm{CsPb}_{3}$ results in poor power conversion efficiencies of only $0.09 \% .{ }^{27}$ Therefore, it is necessary to stabilise the cubic phase of $\mathrm{CsPbI}_{3}$ to allow for its incorporation and encapsulation into a solar cell. In addition, a synthetic method that doesn't require high temperatures would also be a benefital step towards possible commercialisation. Luo et al (2016) developed a low temperature solvent engineering method to convert $\delta-\mathrm{CsPb}_{3}$ in to $\alpha-\mathrm{CsPb}_{3}{ }^{33}{ }^{3} \mathrm{This}$ is done by treating precursor solutions of caesium iodide $(\mathrm{CsI})$ and lead iodide $\left(\mathrm{PbI}_{2}\right)$ in dimethyl formamide (DMF) with $66 \mu \mathrm{L} / \mathrm{mL}$ solution of hydroiodic acid (HI) in water. HI had previously been observed to stabilise the cubic phase of $\mathrm{CsPbI}_{3}$ by inducing the formation of smaller grains ${ }^{34}$ When the treated precursor solution is deposited to form the perovskite film, the HI facilitates the formation of a third crystal structure, a hexagonal perovskite phase $\mathrm{Cs}_{4} \mathrm{PbI}_{6}$. This film is then treated with isopropyl alcohol (IPA) at $100^{\circ} \mathrm{C}$ which converts the $\mathrm{Cs}_{4} \mathrm{PbI}_{6}$ film into $\alpha-\mathrm{CsPb}_{3}$. Constructing solar cells utilising these films produced PCEs up to $4.13 \%$. In addition to the low temperatures required for synthesis, the method also produces films of $\alpha-\mathrm{CsPb}_{3}$ that remain stable for up to 72 hours. ${ }^{33}$

Extraordinarily, the PCEs of $\mathrm{CsPb}_{3}$ based solar cells exceeded $10 \%$ by the end of 2016 . Swarnkar et al (2016) showed that the cubic phase of $\mathrm{CsPbI}_{3}$ can be stabilised far below the normal transition temperature of $320^{\circ} \mathrm{C}$ by minimising the growth of $\alpha-\mathrm{CsPbI}_{3}$ crystals to the nanoscale. ${ }^{35}$ Previous studies had shown that nanocrystals of $\alpha-\mathrm{CsPbI}_{3}$ have superior stability compared to the bulk material. ${ }^{31,36}$ The authors synthesised the perovskite nanocrystals via a hot-injection method that was developed by Protesecu et al (2015), in which a source of $\mathrm{Cs}^{+}$in the form of caesium oleate is injected into a solution of lead (II) iodide $\left(\mathrm{PbI}_{2}\right)$ at $200^{\circ} \mathrm{C}$, resulting in the rapid saturation and precipitation of $\alpha-\mathrm{CsPbI}_{3}$ nanocrystals ${ }^{(31)}$. The nanocrystals were then spin-coated onto mesoporous titanium oxide and incorporated in a solar device using a p-i-n architecture composed of $\mathrm{FTO} /(\mathrm{mp}) \mathrm{TiO}_{2} / \mathrm{CsPbI}_{3} / \mathrm{Spiro}$ $\mathrm{OMeTAD} / \mathrm{MoO}_{\mathrm{x}} / \mathrm{Al}$. The authors reported that their device under reverse scan conditions demonstrated an open-circuit voltage $\left(V_{o c}\right)$ of $1.23 \mathrm{~V}$, a short circuit current $\left(J_{s c}\right)$ of $13.47 \mathrm{~mA} \mathrm{~cm}^{-2}$, a fill factor $(F F)$ of 0.65 , and a PCE of $10.77 \%$. Additionally, the device remained stable for up to 60 days under dry but ambient conditions, demonstrating that the nanoparticles remain stable below the observed phase transition temperature of $\sim 300^{\circ} \mathrm{C}$ for bulk $\mathrm{CsPbI}_{3}$.

By 2018, the PCEs of all inorganic perovskite solar cells had reached 15.07\%. ${ }^{37}$ Wang et al (2018) achieved this result by stabilising $\alpha-\mathrm{CsPb}_{3}$ via the use of $\mathrm{HI}$ and phenylethylammonium iodide 
(PEAI) ${ }^{37}$ As previously discussed, $\mathrm{HI}$ has been observed to reduce the formation energy that is required to achieve the perovskite structure. The PEAI has two roles, to act as a nucleation site for nanocrystal growth and secondly to stabilise the cubic phase and prevent the transition to the orthorhombic phase via the steric bulk of the phenylethyl group. The authors attempted to synthesis their film without the use of $\mathrm{HI}$, but such a method does not result in the desired perovskite phase, demonstrating that $\mathrm{HI}$ is a crucial requirement for lower temperature synthesis of $\alpha-\mathrm{CsPb}_{3}$. Deposition of this HI/PEAI stabilised perovskite film onto mesoporous $\mathrm{TiO}_{2}$ and incorporation into a solar cell with a final architecture of $\mathrm{FTO} / \mathrm{TiO}_{2} / \mathrm{CsPbI}_{3} / \mathrm{HTM} / \mathrm{Au}(\mathrm{HTM}=\mathrm{PTAA})$ resulted in devices that reached an average $\mathrm{PCE}$ of $15.07 \%$ in addition to a $V_{o c}$ of $0.991 \mathrm{~V}, J_{s c}$ of $16.94 \mathrm{~mA} \mathrm{~cm}^{-2}$, and a $F F$ of $68.6 \%$. In addition, the device was exposed to atmospheric conditions at room temperature with humidity levels in the range of $20-30 \%$ and the device maintained $92 \%$ of the initial PCE value even after 60 days of exposure. Also, under a nitrogen atmosphere the perovskite film can be heated up to $225^{\circ} \mathrm{C}$ before mass loss starts to occur.

At the time of writing in 2020, the highest report efficiency of an all inorganic perovskite solar cell is $16.1 \%{ }^{38}$ This value was achieved by improving the quality of the perovskite film and thereby reducing efficiency losses. As with organic-inorganic perovskites, crystallographic defects provide energy levels that can trap charge carriers and facilitate non-radiative recombination in lead trihalide perovskites. Wang et al (2020) aimed to passivate surface defects using the 6TIC-4F molecule (See Figure 3a). ${ }^{38}$

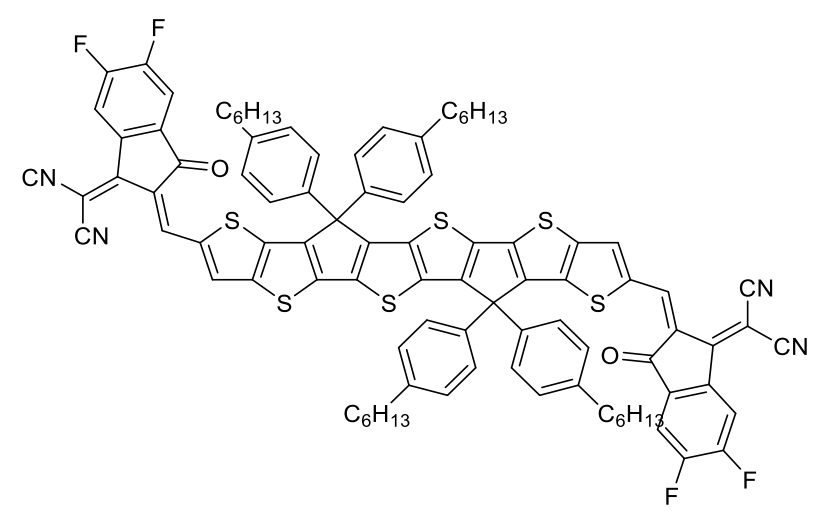

Figure 3a. 6TIC-4F. The cyanide nitrogen atoms can coordinate to and passivate exposed $\mathrm{Pb}^{2+}$ ions on perovskite surfaces.

The 6TIC-4F molecule possess four sp hybridized nitrogen atoms that had been previously theorised to be able to directly coordinate to the $\mathrm{Pb}^{2+}$ cation and therefore coordinate to the exposed surface of a perovskite crystal. In addition, 6TIC-4F possesses electron donating terthieno[3,2-b]thiophene (6 T) groups and two electron withdrawing 2-(5,6-difluoro-3-oxo-2,3-dihydro-1H-inden-1ylidene)malononitrile (IC-2 F). These alternating electron donating and electron withdrawing groups 
facilitate the extraction of electrons from the perovskite and contributed to the performance of the solar device. Not only does this study represent an increase in all inorganic perovskite PCE, but it also demonstrates that the organic components that are used to stabilise the perovskite phase can serve a secondary purpose and directly contribute to device performance.

The significant increase in performance for all inorganic perovskite solar cells suggest that it will not be long till the efficiencies of these devices rival those of their organic-inorganic counterparts. This coupled with their superior thermal stability makes caesium lead halide perovskites an attractive option for the basis of new solar devices. 


\section{General Properties and Characteristics of Lead Halide Perovskites}

The exceptional photovoltaic properties of lead trihalide perovskites include the previously mentioned charge transportation rate, a wide absorption spectrum, exceptional generation of free charge carriers, low rates of radiative and non-radiate recombination, and a high tolerance to crystallographic defects compared to alternative materials such as silicon. As the potential of these materials have become more obvious, researchers have sought to investigate the underlying principles behind their properties so as to inform further developments. Probing the electronic transitions that occur within these materials have produced numerous observations that have helped to inform the current understanding of how charges are generated within a perovskite and why these charges can be transported so effectively even when defects are present within the material.

\section{4a. Optical Absorption Spectrum and Generation of Free Charges}

One factor that contributes to the effectiveness of a lead trihalide perovskite as a light harvesting material is the strong optical absorption of wavelengths across the infrared, visible, and ultra-violet spectrum. This includes wavelengths of light that possess energies right up to and even below the energy value of the band gap. A typical absorption spectrum for a perovskite will include a sharp peak at the absorption onset, which will drop slightly in intensity before steadily and continuously increasing in intensity again. Hetterish et al (2018) investigated the electro absorption spectrum of solutionprocessed perovskite solar cells and analysis of the data via the use of Kramers-Kronig relations and Elliott fits determined that such a spectrum agrees best with excitonic features as opposed to the direct production of free charges. ${ }^{39}$ In essence, the authors determined that the electronic transitions that occur within methylammonium lead trihalide perovskites produce bound electrons and holes that interact with each other to a significant degree as opposed to completely and independent free charges. Specifically, the initial sharp peak is a result of exciton formation, while the gradual increase in absorption intensity is a result of the excitonic continuum, a state in which a single electron is bound to a continuum of holes and vice versa. ${ }^{39}$

The electrostatic interaction between the electron and hole stabilises the pair. Without this stabilising effect, the energy difference between the electron and the hole would be at minimum equal to the band gap between the valence band maximum and conduction band minimum. However, this stabilising effect reduces the energy difference between the pair, and the degree of stabilisation is equal to the strength of the interaction. This brings the energy required to generate the exciton below that of the band gap, explaining why the absorption onset occurs at photon energies just below that of the band gap.

However, for current to be produced from a solar cell, the electrons and holes must accumulate at opposing electrodes. In the case of a typical perovskite solar cell, this requires overcoming the 
electrostatic interaction holding the pair together and separating them before they can recombine. Once sufficiently separated, the excited electrons can travel through an appropriate n-type semiconductor and into an electrode from which they can exit the device and into the external circuit. The holes on the other hand must travel to and through a suitable hole transport material (HTM), such as the commonly used 2, 2', 7, 7'-tetrakis[N, N-di(4-methosyphenyl)amino]-9, 9'-spirofluorene (referred to as either Spiro-OMeTAD or Spiro-MeOTAD), to a counter electrode where they can combine with electrons that are entering the device, completing the circuit. ${ }^{40}$

The exceptional performance of perovskite based solar cells raises the question as to how excitons within the material are so effectively converted into free charges before the processes of recombination can neutralise them. To answer this question required reliably determining the exciton binding energies of a variety of perovskites, as this is the energetic barrier that must be overcome to convert excitons to free charges.

As of the year 2020, multiple studies have estimated the exciton binding energies of a variety of lead trihalide perovskites including methylammonium based perovskites, formamidinium based perovskites, and all inorganic caesium based perovskites. A summary of these binding energies can be viewed in Table $4 a$. What these studies have consistently found is that for these perovskites, the exciton binding energies are comparable to the thermal energy of $25.7 \mathrm{meV}$ that is present at room temperature. This has led to the hypothesis that excitons that are generated within perovskites are able to be ionised by absorbing thermal energy from their surroundings.

\section{Perovskite}

$\mathrm{MAPbBr}_{3}$

$\mathrm{MAPbI}_{3}$

$\mathrm{FAPbBr}_{3}$

$\mathrm{FAPbI}_{3}$

$\mathrm{CsPbCl}_{3}$

$\mathrm{CsPbBr}_{3}$

$\mathrm{CsPbI}_{3}$

\section{Exciton Binding Energy (meV)}

$25^{[5]}-40^{[6]}$

$10^{[6]}-16^{[7]}$

$\sim 22^{[5]}$

$8.1^{[8]}-14^{[5]}$

$\sim 70^{[9]}$

$\sim 40^{[9]}$

$\sim 10^{[9]}$

Table 4a. Commonly discussed lead halide perovskites and the corresponding estimations for the exciton binding energy. $M A=$ Methylammonium, $F A=$ Formamidinium, $C s=$ Caesium.

These conclusions were made based upon studies such as those done by D'Innocenzo et al (2014) in which they modelled the thermodynamic equilibrium between free charges and excitons within methylammonium lead trihalides. These thermodynamic models showed at room temperature 
conditions free charges are overwhelmingly favoured because ambient thermal energy is sufficient to overcome the relatively small binding energy, converting excitons into independent free charges. ${ }^{41}$ This demonstrates that perovskites are more than capable of generating free charges from the bound excitonic states that dominate their absorption spectrum at ambient temperatures. The fact that perovskites can generate bound excitons from low energy photons and readily convert them into free charges is a useful property as it allows for wavelengths of light right up to the band edge to be absorbed and converted into free charges.

\section{4b. Defect Tolerance and Rates of Recombination}

Perovskites demonstrate remarkably low rates of recombination despite the relative simplicity of their synthesis and the defects that result from such methods. For other semiconducting materials, such as silicon, cadmium telluride (CdTe), and copper-indium-gallium selenide ( $\left.\mathrm{CuIn}_{(1-\mathrm{x})} \mathrm{Ga}_{\mathrm{x}} \mathrm{Se}_{2}\right)$, to achieve similar levels of performance requires specific processing methods to maximize grain-size and reduce grain-boundary activity and crystal structure defects. However, lead trihalide perovskites tend to exhibit a tolerance to such defects. As reported by Steirer et al (2016), up to one sixth of the crystal sites within methylammonium lead triiodide $\left(\mathrm{MAPbI}_{3}\right)$ can be vacant while still preserving the significant optoelectronic properties of the material. ${ }^{42}$ This observation results from the energy levels associated with common vacancies falling within the conduction and valence band of the perovskite, preserving the band gap. Hawash et al (2018) used first-principle density-functional theory (DFT) to study the energy levels associated with the various defects that can develop within pure $\mathrm{MAPbI}_{3}$. Their results showed that defects with low formation energies produce energy levels that are less than 0.05 $\mathrm{eV}$ above and below the valence and conduction band respectively. On the other hand, any defects that would produce energy levels deep within the band gap have high formation energies and therefore are hardly present within the material. Based upon their calculations, the dominant electron accepting states result from the vacancy of $\mathrm{Pb}^{2+}$ within the octahedral sites and substitution of $\mathrm{Pb}^{2+}$ with methylammonium $\left(\mathrm{MA}^{+}\right)$within the valence band. The authors explain that the out of phase orbital overlap between the lead $6 \mathrm{~s}$ orbital and the iodide $5 \mathrm{p}$ orbitals raises the valence band maximum above the energy levels of these dominant defects. In addition, the methylammonium cation is simply present to stabilise the overall perovskite phase and does not form any covalent interactions with the lead iodide component, resulting in no additional energy states that may have fallen within the band gap. ${ }^{43}$ This is in stark contrast with semiconductors such as silicon, $\mathrm{CdTe}$, and $\mathrm{CuZnSnS}_{4}$ as similar defects within these materials have associated energy levels that fall within their respective band gaps, adding new localised electronic transitions that do not contribute to the performance of the device.

Though the previously discussed study presented a favourable argument for $\mathrm{MAPbI}_{3}$, their study was based upon a pure perovskite and only investigated the theoretical impact of intrinsic defects, defects that are present within the crystal structure of the material. The incorporation of perovskite films into 
solar devices will inevitably allow contaminates from the other components of the cell into the perovskite film. In addition, the heterojunctions between the perovskite and the charge collecting materials will produce extrinsic defects. Such defects accumulate at the grain boundaries of perovskite crystals and the terminating surfaces, producing energy levels that, depending on their energy level with respect to the valence and conduction bands, can trap free charge carriers. ${ }^{44}$ When in these energy states, the energy of these charge carriers can be lost to the crystal lattice by inducing lattice vibrations, allowing the charges to thermally relax and recombine. This non-radiative recombination process is referred to as Shockley-Read-Hall recombination or trap-assisted recombination and has been observed to be the dominant recombination process that occurs in perovskites solar cells at low charge carrier densities. So, while relatively simple methods of synthesis do allow for the production of respectable solar cells, to maximise the potential of these materials requires minimising the presence of extrinsic defects that allow for trap-assisted recombination to occur. As discussed in earlier chapters, the passivation and reduction of crystallographic defects have resulted in significant leaps in power conversion efficiency for perovskite solar cells, demonstrating why it is crucial to understand the factors that negatively affect lead trihalide perovskites so the research community can address them.

As previously mentioned, at low charge carrier densities, the non-radiative process of trap-assisted recombination dominates over other recombination processes such as the radiative processes of bimolecular recombination and stimulated emission. Since lead trihalide perovskites are direct band gap semiconductors, no significant change in momentum is required to allow for the direct transition of an electron between the conduction and valence bands. This allows for an excited electron to return to the valence band via the emission of a single photon. While such a property can be detrimental to the efficiency of a solar cell, it allows for lead trihalide perovskites to be utilised as light emitting diodes (LEDs). Despite the potential for direct band-to-band radiative recombination, various studies including one by Wehrenfennig et al (2013) have observed remarkably low rates of bimolecular recombination in methylammonium lead trihalide perovskite solar cells. ${ }^{45}$ The authors propose that a possible preferential localisation of electrons and electron holes within the unit cell reduces the spatial overlap of the respective wavefunctions and in turn the rate of recombination. Such an effect would increase the lifetime of charge carriers within the material, providing a large window of time for charge carriers to travel to the appropriate charge carrying material and provide current. However, as of 2020, there is no thorough explanation as to why the rate of radiative recombination is so low at low charge carrier densities, but it is neither the less a useful property. Davies et al (2018) demonstrated that there is a clear correlation between the rate of bimolecular recombination and temperature-dependent changes in the absorption spectra. ${ }^{46}$ This relationship mirrors what is observed in gallium arsenide and silicon and shows that bimolecular recombination in lead trihalide perovskites can be understood as the inverse of absorption. 


\section{Moisture susceptibility and 2D Ruddlesden-Popper Perovskites}

\section{5a. Water Induced Degradation}

Such a rapid increase in performance is a testament to the great potential that perovskite based photovoltaic devices hold. However, this potential is hindered by several factors, one of which is the rapid rate at which these devices degrade when exposed to moisture, heat, and ultra-violet radiation.

Atmospheric moisture is a significant source of lead trihalide perovskite degradation. In essence, a lead trihalide perovskite is a combination of either an organic or inorganic monohalide salt and a lead dihalide salt. Exposure to moisture allows for the development of an equilibrium between the perovskite phase and the two precursor salts because water molecules are able to overcome the ionic forces that hold the monovalent cation and the lead halide octahedra together.

A plausible two step degradation pathway for methylammonium triiodide was suggested by Frost $e t$ al (2014), shown below in Figure $5 \boldsymbol{a ~} .^{47}$ In this process, it is proposed that a single water molecule catalyses the deprotonation of methylammonium and the extraction of iodine. Excess water dissolves the volatile and water soluble methylamine $\left(\mathrm{CH}_{3} \mathrm{NH}_{3}\right)$ and hydrogen iodide (HI) leaving just the remaining lead iodide $\left(\mathrm{PbI}_{2}\right)$. The water molecule is released and is capable of degrading more perovskite. The authors state that this degradation process would continue until the excess water is saturated in HI or the vapour pressure of $\mathrm{CH}_{3} \mathrm{NH}_{2}$ is at equilibrium. Otherwise, the process will continue until the whole perovskite has been destroyed. ${ }^{47}$

However, it has since been observed that the exposure of a lead trihalide perovskite results in the formation of several different hydrated species. Such hydrates have been known of since at least 1987, when Vincent et al (1987) discovered that the black methylammonium lead triiodide $\left(\mathrm{CH}_{3} \mathrm{NH}_{3} \mathrm{PbI}_{3}\right)$ would convert to a yellow compound which they later identified as $\left(\mathrm{CH}_{3} \mathrm{NH}_{3}\right)_{4} \mathrm{PbI}_{6} \cdot 2 \mathrm{H}_{2} \mathrm{O}$. ${ }^{48}$ The compound possesses a distorted $\mathrm{NaCl}$-type lattice consisting of individual $\mathrm{PbI}_{6}{ }^{4-}$ octahedral units separated from each other by methylammonium water dimers $\left(\mathrm{CH}_{3} \mathrm{NH}_{3} \cdots \mathrm{H}_{2} \mathrm{O} \cdots \mathrm{CH}_{3} \mathrm{NH}_{3}\right)_{2}{ }^{4+}{ }^{48}$ It had been proposed that the initial exposure to moisture would result in the conversion of methylammonium lead triiodide into this dehydrate (see Figure $5 \boldsymbol{a}$.), followed by further degradation. However, Leguy et al (2015) disproved this hypothesis and found that a mono-hydrate forms first, followed by further degradation into the dihydrate (see Figure 5a.). ${ }^{49}$ While each step in this degradation process is technically reversible, the presence of excess water forces the forward reaction to be favoured and result in the complete degradation of the perovskite. ${ }^{49}$ 
(A)

(B)

(D)

$$
\left[\left(\mathrm{CH}_{3} \mathrm{NH}_{3}\right) \mathrm{PbI}_{3}\right]_{n}+\mathrm{H}_{2} \mathrm{O} \rightleftharpoons\left[\left(\mathrm{CH}_{3} \mathrm{NH}_{3}\right)_{n-1}\left(\mathrm{PbI}_{3}\right)\right]_{n}\left[\mathrm{H}_{3} \mathrm{O}^{+}\right]+\mathrm{CH}_{3} \mathrm{NH}_{2}
$$

$$
\left[\left(\mathrm{CH}_{3} \mathrm{NH}_{3}\right)_{n-1}\left(\mathrm{PbI}_{3}\right)\right]_{n}\left[\mathrm{H}_{3} \mathrm{O}^{+}\right] \rightleftharpoons \mathrm{HI}+\mathrm{PbI}_{2}+\left[\left(\mathrm{CH}_{3} \mathrm{NH}_{3}\right) \mathrm{PbI}_{3}\right]_{n-1}+\mathrm{H}_{2} \mathrm{O}
$$

$$
4 \mathrm{CH}_{3} \mathrm{NH}_{3} \mathrm{PbI}_{3}+2 \mathrm{H}_{2} \mathrm{O} \rightleftharpoons\left(\mathrm{CH}_{3} \mathrm{NH}_{3}\right)_{4} \mathrm{PbI}_{6} \cdot 2 \mathrm{H}_{2} \mathrm{O}+3 \mathrm{PbI}_{2}
$$

$$
4 \mathrm{CH}_{3} \mathrm{NH}_{3} \mathrm{PbI}_{3}+4 \mathrm{H}_{2} \mathrm{O} \rightleftharpoons 4\left[\mathrm{CH}_{3} \mathrm{NH}_{3} \mathrm{PbI}_{3} \cdot \mathrm{H}_{2} \mathrm{O}\right] \rightleftharpoons\left(\mathrm{CH}_{3} \mathrm{NH}_{3}\right)_{4} \mathrm{PbI}_{6} \cdot 2 \mathrm{H}_{2} \mathrm{O}+3 \mathrm{PbI}_{2}+2 \mathrm{H}_{2} \mathrm{O}
$$

Figure 5a. (A and B) The two step acid-base degradation process proposed by Frost et al. (C) The initial proposal for the formation of $\left(\mathrm{CH}_{3} \mathrm{NH}_{3}\right)_{4} \mathrm{PbI}_{6} \cdot 2 \mathrm{H}_{2} \mathrm{O}$ from $\mathrm{CH}_{3} \mathrm{NH}_{3} \mathrm{PbI}_{3}$. (D) The final pathway for the formation of $\left(\mathrm{CH}_{3} \mathrm{NH}_{3}\right)_{4} \mathrm{PbI}_{6} \cdot 2 \mathrm{H}_{2} \mathrm{O}$ from $\mathrm{CH}_{3} \mathrm{NH}_{3} \mathrm{PbI}_{3}$.

Moisture induced degradation represents one of the major hurdles to the commercialisation of lead trihalide perovskite solar cells. Currently the technology demands anhydrous conditions for not only the fabrication of these devices but also for their operation. This significant draw back has led to researchers to investigate the structurally related 2D Ruddlesden-Popper perovskites as it has been observed that these materials display a greater tolerance to the presence of moisture.

\section{5b. Introducing 2D Ruddlesden-Popper Perovskites}

2D Ruddlesden-Popper perovskites are characterised by their layered structure of alternating inorganic and organic phases. Such a structure is stabilised by incorporating long chain organic molecules with protonated amine groups into some or all of the A sites within the perovskite crystal structure. The long organic chains separate the $\mathrm{PbX}_{6}{ }^{4-}$ octahedra in one dimension, isolating them to a two dimensional sheet. When all the A sites are replaced with large organic molecules, the lead halide octahedra are completely isolated in the third dimension and are only a few atoms thick. The dimensionality of the inorganic phase can be increased by re-incorporating small monovalent cations into the structure, allowing multiple layers of $\mathrm{PbX}_{6}{ }^{4-}$ to come into direct contact (see Figure 5b). The hydrophobic nature of these large organic cations acts to hinder moisture from reaching the inorganic layers and therefore reduce the rate of degradation. 

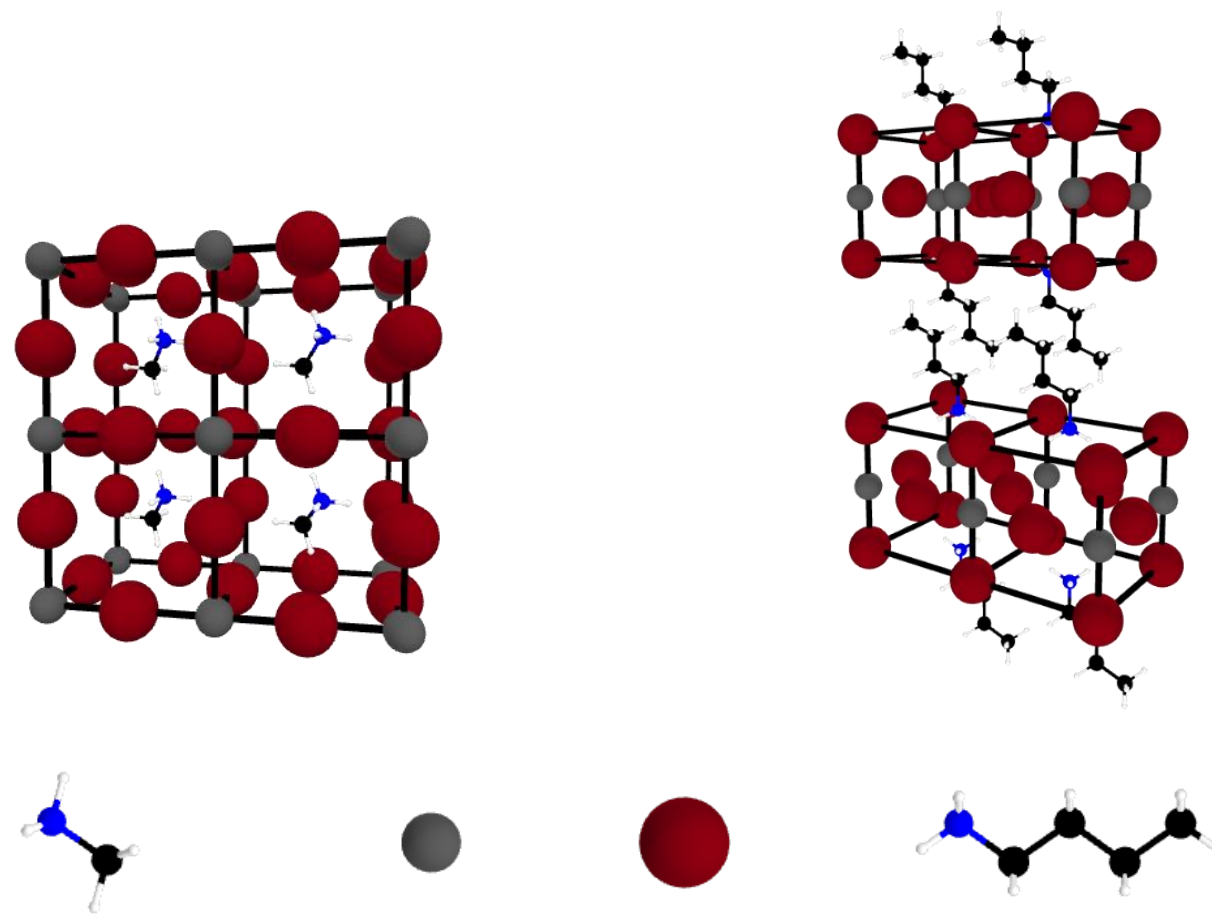

Methylammonium $\left(\mathrm{MA}^{+}\right)$

Lead $\left(\boldsymbol{P b}^{2+}\right)$

Iodide (I)

Butylammonium $\left(\mathrm{BA}^{+}\right)$

Figure 5b. Left: A diagram of a three dimensional perovskite. Right: Diagram of a pure two dimensional Ruddlesden-Popper perovskite.

The first application of such a material in the field of photovoltaics was done by Karunadasa et al (2014), utilising the layered perovskite $\left(\mathrm{C}_{6} \mathrm{H}_{5}\left(\mathrm{CH}_{2}\right)_{2} \mathrm{NH}_{3}\right)_{2}\left(\mathrm{CH}_{3} \mathrm{NH}_{3}\right)_{2} \mathrm{~Pb}_{3} \mathrm{I}_{10}$ (or $\left[(\mathrm{PEA})_{2}(\mathrm{MA})_{2} \mathrm{~Pb}_{3} \mathrm{I}_{10}\right]$ ) in a p-n junction device using fluorine doped tin oxide (FTO), titanium dioxide $\left(\mathrm{TiO}_{2}\right), 2,2^{\prime}, 7,7^{\prime}$ tetrakis-( $N, N$-di-p-methoxyphenylamine) $9,9^{\prime}$-spirobifluorene (spire-OMeTAD), and gold. ${ }^{50}$ The authors reported that devices based upon this architecture produced open circuit voltages $\left(V_{O C}\right)$ above $1.0 \mathrm{~V}$ and up to a max of $1.18 \mathrm{~V}$, a short-circuit current of $6.72 \mathrm{~mA} \mathrm{~cm}^{2}$, a fill factor $(F F)$ of 0.60 , and a power conversion efficiency (PCE) of $4.73 \%$. While these initial efficiency values were low compared to the values produced by equivalent devices utilising standard three-dimensional perovskites, the device did display a greater resistance to moisture, remaining stable when exposed to a relative humidity level of $52 \%$ for 46 days, confirming the influence of the large organic cations on the materials moisture susceptibility. ${ }^{50}$

Like their three dimensional counterparts, the power conversion efficiencies of these devices have sharply increased, reaching $12.52 \%$ in 2016 and as high as $18.06 \%$ in $2020 .{ }^{51-52}$ Moisture resistance has also increased, with operational lifetimes on the order of thousands of hours when exposed to high humidity and constant illumination conditions with little degradation (retaining $85 \%$ of their initial power conversion efficiency) being reported. Such progress creates a strong argument for 2D 
Ruddlesden-Popper perovskites as the basis of the next generation of perovskite based solar cell devices.

\section{5c. From Three to Two Dimensions, How the Electronic Properties Change}

Besides increasing their resistance to moisture, the introduction of large organic cations and the reduction in the dimensionality of the semiconducting inorganic phase has significant consequences with regards to the photovoltaic properties of the material. The reduction in dimensionality produces a quantum confinement effect in the semiconducting $\mathrm{PbX}_{6}{ }^{4-}$ layers. As the thickness of the inorganic phases is reduced, there eventually comes a point where the thickness is comparable to the de Broglie wavelength of the electron. At this point, the inorganic phase acts as a quantum well with discrete energy levels that charge carriers can occupy. As the size of the well decreases the energy of these excited states along with the energy separation between them increases. This increased separation between energy states also applies to the valance maximum and conduction band minimum, resulting in a larger band gap. Therefore, to induce a photoexcitation event in low dimensional semiconductors requires higher energy wavelengths. As a consequence, the band gap of $2 \mathrm{D}$ ruddlesden-popper perovskites tend to be less ideal than their three dimensional counterparts.

Confining the inorganic phases between the organic layers in one direction has also been found to significantly increase the exciton binging energies within these materials. Excitons that are generated within the inorganic phase will be confined to that specific layer due to the significantly lower dielectric constant of the organic phase coupled with the previously discussed quantum confinement effect. This keeps the electrons and holes together and therefore increases the energy barrier that must be overcome to separate them. For example, Yaffe et al (2015) fabricated solar cells utilising the perovskite $\left(\mathrm{C}_{4} \mathrm{H}_{9} \mathrm{NH}_{3}\right)_{2} \mathrm{PbI}_{4}\left(\mathrm{BA}_{2} \mathrm{PbI}_{4}\right)$ and measured the band gap to be $2.4 \mathrm{eV}$ and the exciton binding energy to be $490 \mathrm{meV} .{ }^{53}$ Such a high binding energy far exceeds the thermal energy found at room temperature of around $25.7 \mathrm{meV}$ and therefore prevents the effective conversion of excitons into free charge carriers. The result of this is that the bimolecular recombination rate within 2D ruddlesden-popper perovskites can be significantly greater than in $3 \mathrm{D}$ perovskites. While this is a detrimental characteristic for utilisation in solar cells, a high rate of radiant recombination is a positive characteristic for applications in light emitting diodes. For example, Ying et al (2017) utilised the 2D ruddlesden-popper perovskite naphthylmethylammonium formamidinium lead iodide $\left(\mathrm{NMA}_{2} \mathrm{FAPb}_{2} \mathrm{I}_{7}\right)$ and found that the rate of bimolecular recombination is greater, allowing for more efficient lead halide perovskite based electroluminescent devices. ${ }^{54}$

Another consideration is charge transport. As mentioned previously, the low dielectric constant of the organic cations confine charge carriers within the inorganic layer they were generated and cannot easily pass from one semiconducting layer, through the insulting organic layer, into another inorganic layer. 
Therefore, if a 2D ruddlesden-popper perovskite was incorporated into a solar device in such a way that the perovskites layers were orientated in parallel with respect to the other layers of the device, then charges generated deep within the perovskite film would be unable to travel vertically through the film to reach those substrates. Therefore, to maximise the efficiency of a solar cell that utilises a 2D ruddlesden-popper perovskite requires orientating the layers of the perovskite to be perpendicular to the substrate so as to act as charge transport channels. Such orientation is critical to device operation as has been demonstrated in several publications. ${ }^{51-52}$
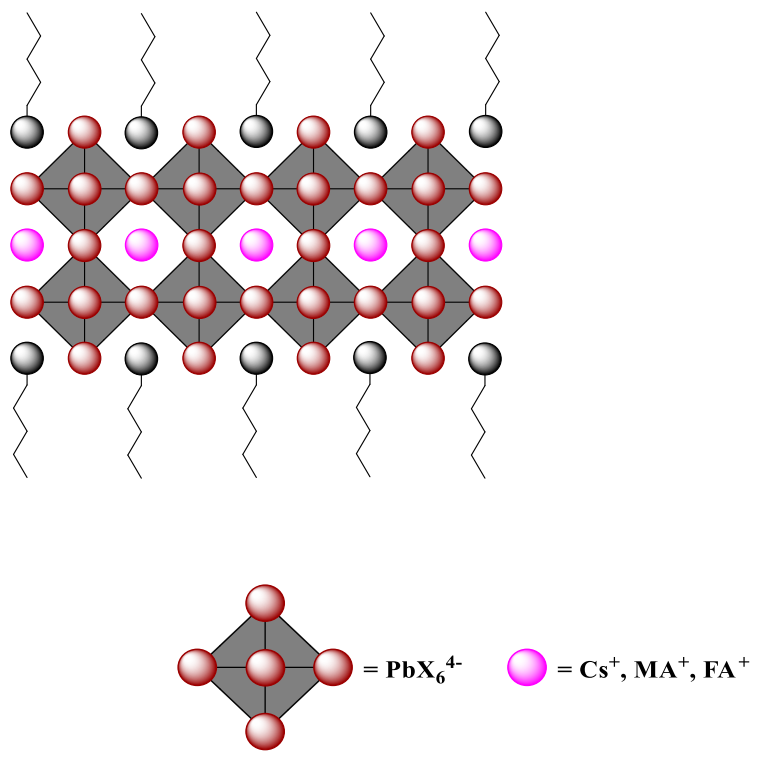
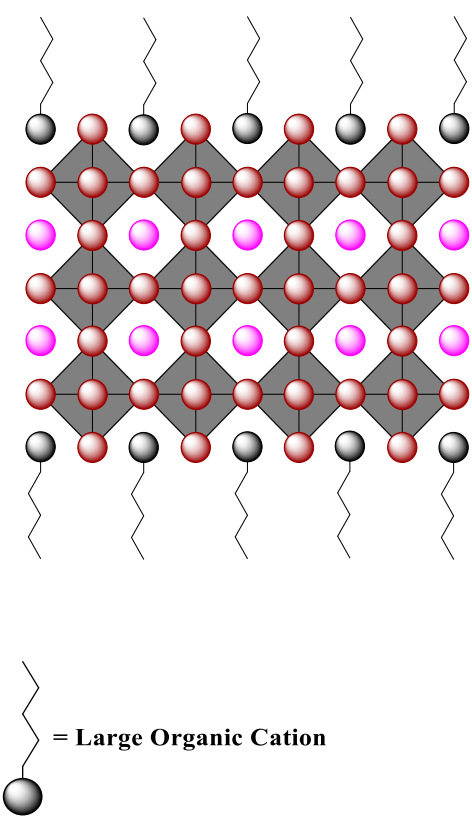

Figure 5c. left: A 2D/3D Hybrid Ruddlesden-Popper Perovskite with $n=2$ lead halide layers. Right: A 2D/3D Hybrid Ruddlesden-Popper Perovskite with $n=3$ lead halide layers.

\section{5d. 2D/3D hybrid Perovskites, The Best of Both Worlds}

As is with other characteristics of a lead halide perovskite, the thickness of the inorganic phase of a perovskite can be tuned to produce a 2D/3D hybrid (Figure $5 \boldsymbol{c}$.). This is done by altering the chemical composition with respect to the A cations. In fact, as previously discussed, the first solar device that utilised a low dimensional perovskite by Karunadasa et al (2014) utilised a 2D/3D perovskite. ${ }^{50}$ The perovskite, $(\mathrm{PEA})_{2}(\mathrm{MA})_{2} \mathrm{~Pb}_{3} \mathrm{I}_{10}$, possesses inorganic layers that are three $\mathrm{PbI}_{6}{ }^{4-}$ octahedra thick $(n=3)$. Such a structure is stabilised by adding both the large phenethylammonium cations and the small methylammonium cations, allowing for the stabilisation of a traditional three dimensional perovskite phase but confining it's thickness to only three layers. The authors chose this thickness as estimations showed this would be sufficient to reduce the quantum confinement effect and bring the band gap down to around $2.1 \mathrm{eV}$, as opposed to a band gap of $2.35 \mathrm{eV}$ for the pure two dimensional perovskite 
(PEA) $)_{2} \mathrm{PbI}_{4}(n=1)$. In addition, absorption measurements showed an exciton peak at $2.06 \mathrm{eV}$, only 0.04 $\mathrm{eV}$ below the estimated band gap. ${ }^{50}$ This result provided evidence that a mild increase in dimensionality is enough to reduce the exciton binding energy to values that are comparable to conventional three dimensional perovskites.

Other theoretical studies have also produced results that suggest that a relatively minor increase in dimensionality will reduce the excitonic binding energy and therefore facilitate the effective conversion of excitons to free carriers by room temperature thermal energy. Tsai et al (2016) stated that based on their own DFT calculations modelling the electronic properties of the perovskites $(B A)_{2}(\mathrm{MA})_{2} \mathrm{~Pb}_{3} \mathrm{I}_{10}$ $(n=3)$ and $(\mathrm{BA})_{2}(\mathrm{MA})_{3} \mathrm{~Pb}_{4} \mathrm{I}_{13}(n=4)$ that the exciton binding energies would be comparable to three dimensional perovskites. ${ }^{51}$ Experimental optical absorption data of $(\mathrm{BA})_{2}(\mathrm{MA})_{3} \mathrm{~Pb}_{4} \mathrm{I}_{13}(n=4)$ showed no excitonic features, indicating that free charges were dominant within the material. ${ }^{51}$ Koutselas et al (1996) also calculated the exciton binding energies for several low dimensionality perovskites with different $n$ values. The results of their work also show that an increase in dimensionality results in a decreasing exciton binding energy (see Table 5a). ${ }^{55}$. The previously discussed theoretical studies demonstrate that through appropriate compositional engineering, the high photovoltaic performance traditional 3D perovskites can be exhibited in low dimensional perovskites.

\section{Compound}

$\mathrm{MAPbI}_{3}$

(NA) $)_{2}(\mathbf{M A})_{3} \mathbf{P b}_{4} \mathbf{I}_{13}$

$(\mathrm{NA})_{2}(\mathrm{MA})_{2} \mathbf{P b}_{3} \mathbf{I}_{10}$

$(\mathrm{NA})_{2}(\mathrm{MA})_{1} \mathrm{~Pb}_{2} \mathbf{I}_{7}$

$(\mathrm{NA})_{2} \mathrm{PbI}_{4}$
Dimensionality

$3 \mathrm{D}$

2D (Four Layers)

2D (Three Layers)

2D (Two Layers)

2D (One Layer)
Band Gap

(eV)

1.70

2.03

2.17

2.38

$\geq 2.82$
Exciton Binding Energy

(meV)

30

60

96

181

$\geq 388$

Table 5a. Summary of band gaps and exciton binding energies for several organic/inorganic lead halide perovskites. Values were estimated by Koutselas et al (1996) based upon optical absorption measurements. ${ }^{55} \mathrm{MA}=$ Methylammonium $\left(\mathrm{CH}_{3} \mathrm{NH}_{3}{ }^{+}\right.$and $\mathrm{NA}=$ Nonylammonium $\left(\mathrm{CH}_{3}\left(\mathrm{CH}_{2}\right)_{8} \mathrm{NH}_{3}{ }^{+}\right)$.

2D Ruddlesden-Popper perovskites offer the potential of a more durable and commercially viable perovskite based solar cells. While pure 2D perovskites possess unfavourably high band gaps and excitonic binding energies, compositional engineering allows for the production of hybrid perovskites that possess both the desired physical properties of three dimensional perovskites but also the stability of two dimensional perovskites. Further research and optimisation will be needed to allow the 
technology to catch up to equivalent three dimensional perovskite solar cells and already established photovoltaic technology. 


\section{Utilising Organic Molecules to Overcome the Shockley-Queisser Limit}

The successful application of 2D Ruddlesden-Popper perovskites opens new avenues for innovation that would not be possible with traditional three dimensional perovskites. Currently, most 2D and 2D/3D hybrid perovskites utilise the large organic spacer cations simply to increase the stability of the perovskite with regards to moisture and temperature. As such, the organic spacer cations do not contribute to the light harvesting potential of the bulk material. Through the addition of fluorescent functional groups, these organic phases could be utilised to enhance the fluorescent properties of lead halide perovskites.

\section{6a. Energy Transfer between Organic Molecules and Inorganic Semiconductors}

Semiconducting nanomaterials have quickly developed into an important class of materials for a variety of applications such as photovoltaics, photochemistry, and biomedicine. However, these materials have their limitations. To overcome these limitations, researchers have turned to coordinating semiconducting nanomaterials to certain organic molecules in attempts to funnel energy into or out of those nanomaterials.

There are multiple processes that allow for the transfer of energy from organic fluorophores to inorganic semiconductors. One method of energy transfer is via the process of förster resonance energy transfer (FRET). FRET requires two light sensitive species, whether they be chromophores or nanoparticles. One of the pair acts as a donor, the species that absorbs the initial incident light, while the other molecule is the acceptor which receives that harvested energy from the donor and performs the final re-emission. For the donor to transfer energy to the acceptor, the emission from the donor must emit a significant range of wavelengths that can be absorbed by the acceptor. In other words, there must be significant overlap between the emission spectrum of the donor and the absorption spectrum of the acceptor. If this is the case, then the dipole moments of the two species can couple and facilitate the transfer of energy without the emission or absorption of photons. This process returns the donor to its electronic ground state while acceptor is excited to a higher electronic energy state. Another method of energy transfer is Dexter Energy Transfer, which is a process that involves the exchange of electrons between a chemical species within an electronic excited state and another chemical species in its ground state. For the electron exchange to take place, their needs to be not only a spectral overlap between the emission of the donor and the absorption of the acceptor, but also an overlap of wavefunctions. Such a requirement requires that the donor and acceptor are separated by no more than 10 angstroms.

The coupling of different light sensitive materials offers potential avenues to circumventing the Shockley-Queisser limit. The limit, as previously mentioned, is based upon a simple single junction solar cell that utilises a single light absorbing material with a single band gap. The result of such an architecture are spectrum losses which represents the largest source of energy loss. There are two 
processes that when coupled with Dexter Energy Transfer are capable of reducing spectral losses. These process are triplet-triplet annihilation and singlet fission.

Triplet-triplet annihilation (TTA) is the process in which the energy of two molecules in triplet excited states are combined to generate a single higher energy state in one of those two molecules (see Figure $\mathbf{6 a}$ ). Such a process allows two photons to be utilised in conjunction to produce a single energy state that can emit one high energy photon. Therefore up-conversion offers a way of utilising wavelengths of light that possess energies below than what is required and convert them into usable high energy photons. This process has been utilised in conjunction with various inorganic semiconducting materials. For example, Huang et al (2015) coupled lead selenide ( $\mathrm{PbSe}$ ) nanocrystals with rubrene based ligands and showed via time resolved fluorescence spectroscopy that the illumination of the PbSe-Rubrene system with $980 \mathrm{~nm}$ infrared light resulted in a delayed fluorescence signal at $550 \mathrm{~nm} .{ }^{56}$ The authors attributed this delay to the annihilation of triplet excited states within the rubrene ligands. These excited states were induced by the transfer of energy from the inorganic nanoparticles. These nanoparticles absorbed the initial $980 \mathrm{~nm}$ light, which generated charge carriers within the nanoparticle that could couple with the dipole moment of the organic ligand, allowing energy transfer. ${ }^{56}$ Similarly, Mongin et al (2016) demonstrated the transfer of energy from cadmium selenide nanoparticles to anthracene and pyrene based ligands. ${ }^{57}$ Illumination of the modified nanoparticles with $505 \mathrm{~nm}$ light produced an identifiable quenching of

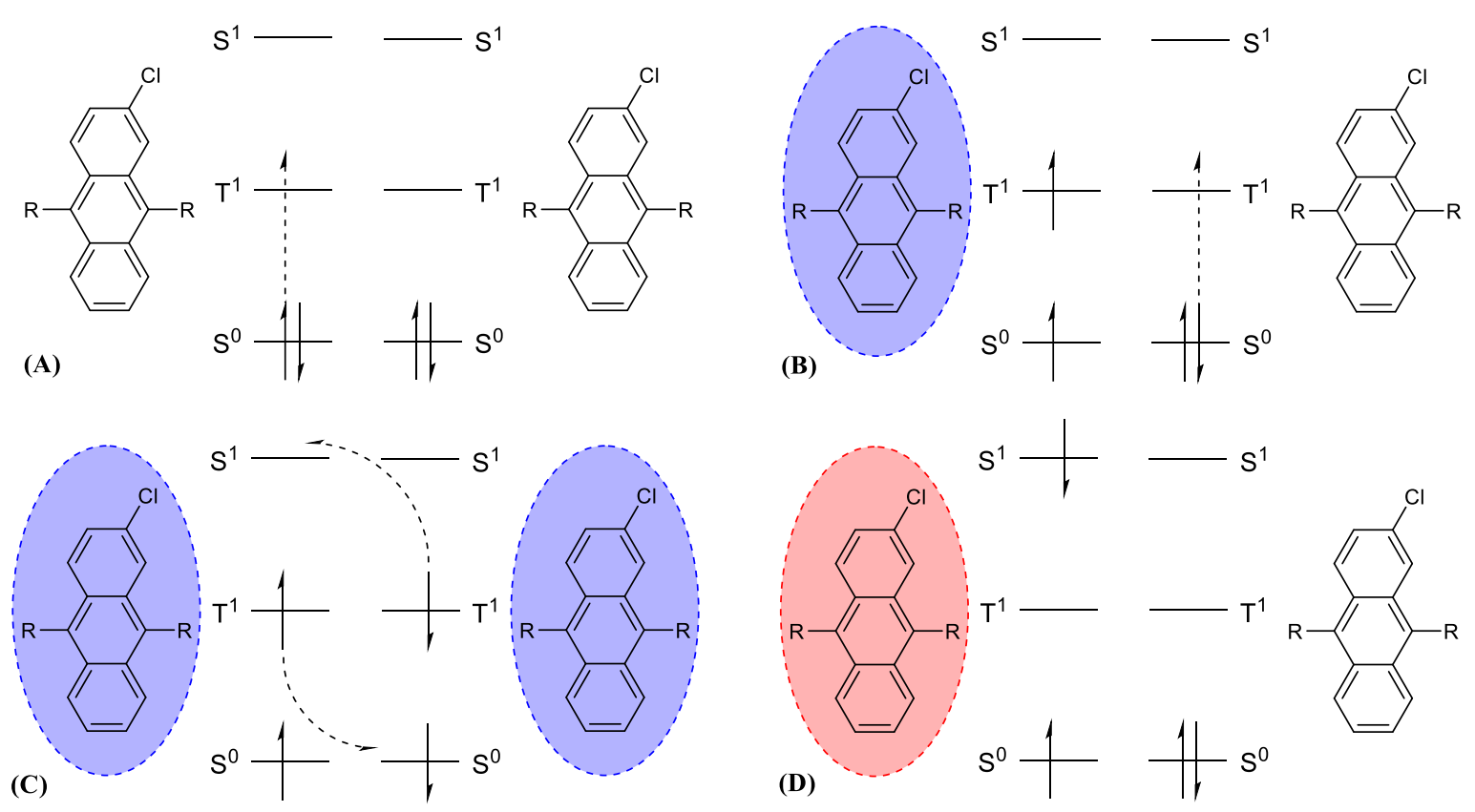

Figure 6a. Triplet-triplet annihilation (TTA) in 2-chloro-9,10-bis(phenylethynyl)anthracene (CBPEA). (A) and (B) Sequential generation of two excited triplet states $\left(T^{I}\right)$. (C) and (D) TTA and the generation of one singlet excited state. $R=$ Phenylethynyl $\left(-C \equiv C-C_{6} H_{5}\right)$. 
nanoparticle emission by the organic chromophores. Unlike the previous example, the surface bound chromophores did not perform the triplet-triplet annihilation, but instead further transferred the energy to another chromophore, 2-chloro-9,10-bisphenylethynylanthracene (CBPEA) to perform triplet-triplet annihilation. ${ }^{57}$

The second process, singlet fission (SF), can occur in a molecule that possesses a triplet excited state that, in terms of energy level, is comparable to half that of the same molecules singlet excited state. When such a molecule is promoted into a singlet excited state, the energy of that single state can be shared equally between two molecules, inducing triplet excited states within each molecule (see Figure $\boldsymbol{6 b})$. While the conversion of a singlet state into a triplet state is spin forbidden due to the change in overall spin, singlet fission is spin allowed as the two molecules are initially spin coupled and will possess countering spins, resulting in no overall change in spin for the system before and after the process has occurred. Singlet fission offers a potential method of reducing thermalisation losses by taking photons that, without any intervention, would only be capable of generating a single excitation event and splitting them into two photons that are each capable of excitation.

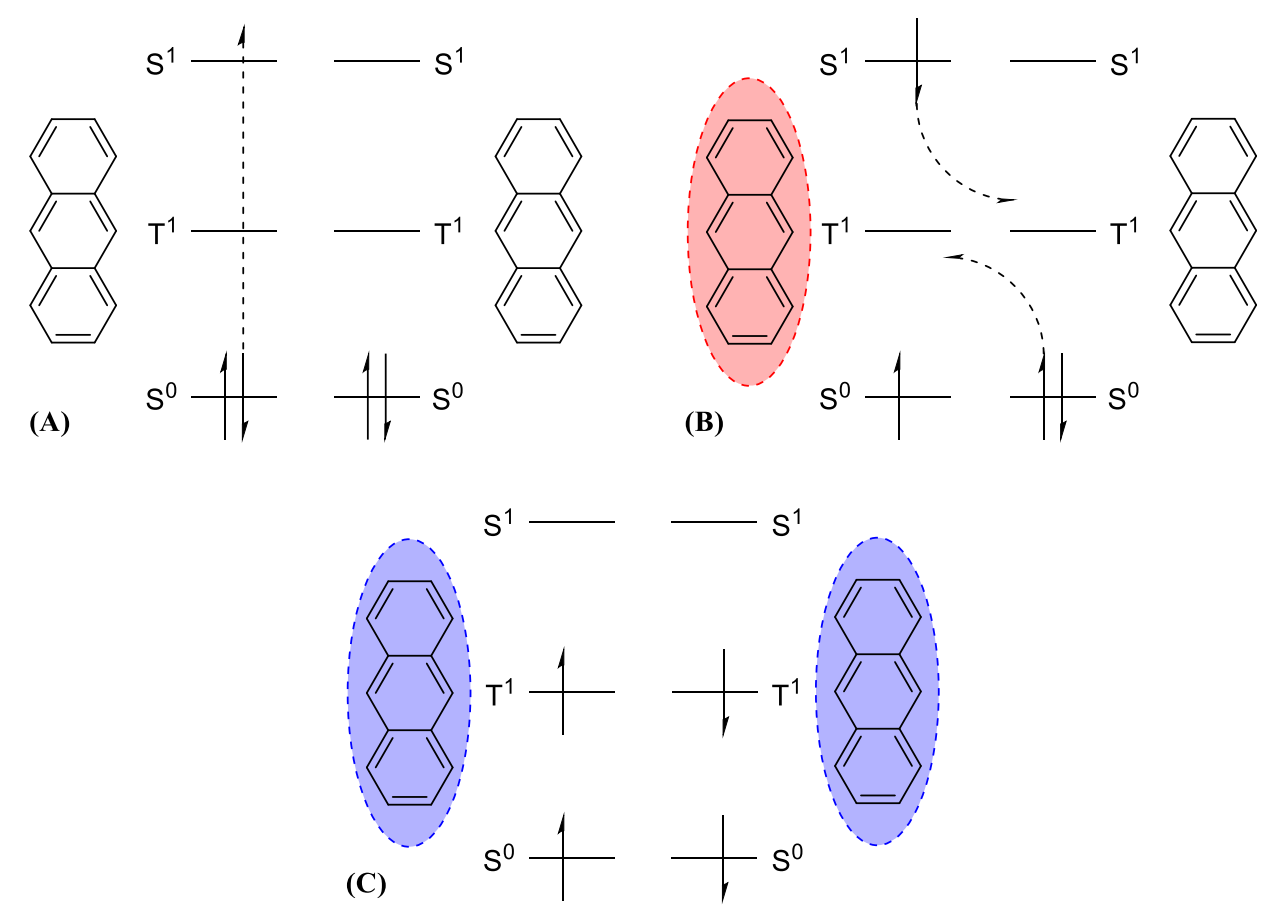

\footnotetext{
Figure 6b. Singlet Fission (SF) in Anthracene. (A) Singlet state generation. (B) and (C) SF and the generation of two triplet excited states.
}

There are two types of singlet fission, exothermic and endothermic. Exothermic singlet fission is a rapid process with a high conversion efficiency while endothermic singlet fission is a less effective process as it has to compete with intersystem crossing and recombination processes. However, endothermic singlet fission is an energetically uphill process and produces triplet states with a greater 
combined energy than the original singlet state. In addition, most molecules that are capable of exothermic singlet fission result in triplet excited states that are low in energy and cannot be coupled with existing semiconducting technologies. Molecules that are capable of endothermic singlet fission include anthracene and tetracene. More importantly, the triplet excited states of these molecules are around 1.78 and $1.2 \mathrm{eV}$ respectively. Such values are within the range of band gap values for semiconducting materials such as silicon $(1.12 \mathrm{eV})$ and certain lead halide perovskites.

Examples of down conversion being utilised include work by Ehrler et al (2012), who demonstrated the utilisation of singlet fission to generate two excitons from individual high energy photons ${ }^{58}$. The group utilised lead sulfide $(\mathrm{PbS})$ nanocrystals as an infrared absorbing semiconductor which they size tuned to produce a band gap of $0.7 \mathrm{eV}$. Such an energy value is comparable to the triplet state of the fluorophore pentacene, an acene molecule capable of rapid exothermic singlet fission. Depositing the pentacene onto an indium tin oxide (ITO) glass slide, followed by the lead sulfide nanocrystals and an aluminium electrode. An external quantum efficiency spectrum showed that photocurrent was being generated from both the pentacene and the lead sulfide nanocrystals. Internal quantum efficiency measurements showed a small increase in efficiency when absorbing in the pentacene absorption region, indicating that the presence of pentacene allowed for a greater number of charges to be generated from $\mathrm{PbS}$ nanocrystals when absorbing light in this region. However, the power conversion efficiency of these devices only reached a maximum PCE value of $0.85 \% .{ }^{58}$

While the process of down-converting light into multiple excitation events is possible and efficient, harvesting of triplet excitons has proven to be an inefficient process. To circumvent this issue, an alternative method of utilising triplet energy states is to convert them into emitted photons. These photons could then be reabsorbed by another material to generate singlet excitons that can be more efficiently converted into free charges. This is the basic concept behind a singlet fission photon multiplier. Davis et al (2018) sought to test this concept by coupling carboxylic acid-functionalised, singlet fission capable, 5,12-bis((triisopropylsilyl)ethynyl)tetracene (TIPs-tetracene) molecules to $\mathrm{PbS}$ quantum dots ${ }^{59}$. Analysis of this system showed that a photon multiplier such as this does allow for multiple emission events to be generated from individual high energy photons. Targeted excitation of the tetracene molecules resulted in quantum dot emission at $1250 \mathrm{~nm}$ with a photoluminescence quantum yield (PLQE) of 17\%. Targeted excitation of the quantum dots outside the absorption range of the ligands also resulted in emission but with a PLQE of only $9 \% .{ }^{59}$ Such a result confirms that for each photon absorbed by the tetracene ligands, almost two excitation events are transferred to and emitted by the quantum dots.

Experiments such as these prove that organic molecules can be utilised in conjunction with inorganic semiconductors to result in enhanced photoluminescent and photovoltaic properties. However, there has been little work done with regards to the coupling of organic chromophores and perovskites. 


\section{6b. Anthracene}

Anthracene is a polycyclic aromatic hydrocarbon, consisting of three fused benzene ring units (see Figure 6c). Anthracene is also a semiconductor with a wide band gap of $3.72 \pm 0.03 \mathrm{eV}$, which corresponds to a wavelength of 330 to $336 \mathrm{~nm} .{ }^{60}$ With a first excited triplet state energy of $1.78 \mathrm{eV}$, singlet fission that occurs within anthracene is an endothermic process as the total energy of the product triplet states is greater than that of the original singlet excited state. Investigations into delayed fluorescence in anthracene lead to the initial development of singlet fission as a concept. While the rates of singlet fission that occurs within anthracene is extremely low at around 3\% efficiency, appropriate functionalisation of the molecule can influence the electronic properties of the polyaromatic system and increase this rate. ${ }^{61}$ Bae et al (2018) added phenylethynyl groups to the 9 and 10 positions of the molecule and found this reduced the triplet excited state to $\sim 1.2 \mathrm{eV}$ while leaving the singlet excited state unaltered. ${ }^{62}$ In addition, such an energy state correlates well with the ideal band gap of $1.1 \mathrm{eV}$.

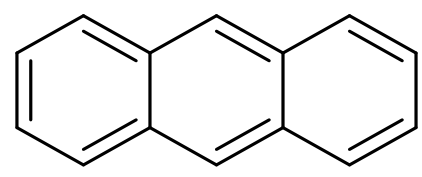

Figure 6c. The skeletal structure of the Anthracene molecule, $\mathrm{C}_{14} \mathrm{H}_{10}$.

However, while the triplet excited state for unaltered anthracene is not optimised for semiconducting technologies such as for silicon, it is currently appropriate for perovskites. At the time of writing, the lowest band gap value for a lead halide perovskite is $1.48 \mathrm{eV}$ for formamidinium lead triiodide, and 2D Ruddlesden-Popper perovskites possess band gaps that are even higher in energy. Therefore, anthracene is an appropriate fluorophore to test whether energy transfer can occur between organic fluorophores and lead halide perovskites, especially with regards to the process of singlet fission.

To couple anthracene to lead halide perovskites requires functionalising the molecule to enable it to coordinate to the inorganic material. The carboxylic acid functional group is useful for multiple purposes. Firstly, deprotonated carboxylic acids are capable of coordinating to the surface of nanoparticles, allowing for the coordination of molecules to stabilise and even functionalise the surface of the particle. Secondly, carboxylic acids are good targets for further modification. For example, the carboxylic acid functionalised anthracene molecule can be reacted with a diamine, resulting in a derivative that possesses a terminal amine group (see Figure 4c). Amines, like carboxylic acids, are utilised in the stabilisation and functionalisation of nanoparticles. Additionally, protonated amines can be incorporated into the crystal structure of lead halide perovskites. Therefore, a carboxylic acid functionalised anthracene derivative offers many possible ways to couple anthracene to a lead halide perovskite. 


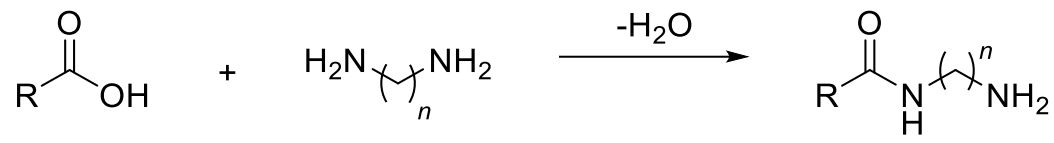

\section{Figure 6d. The conversion of a carboxylic acid to an amide.}

If a polyaromatic such as anthracene can be coupled to lead halide perovskites that possess comparable bandgap energies to the triplet energy state of anthracene, it would open up avenues for circumventing the Shockley-Queisser limit and further increasing the performance of perovskite based photovoltaic devices. 


\section{Aim}

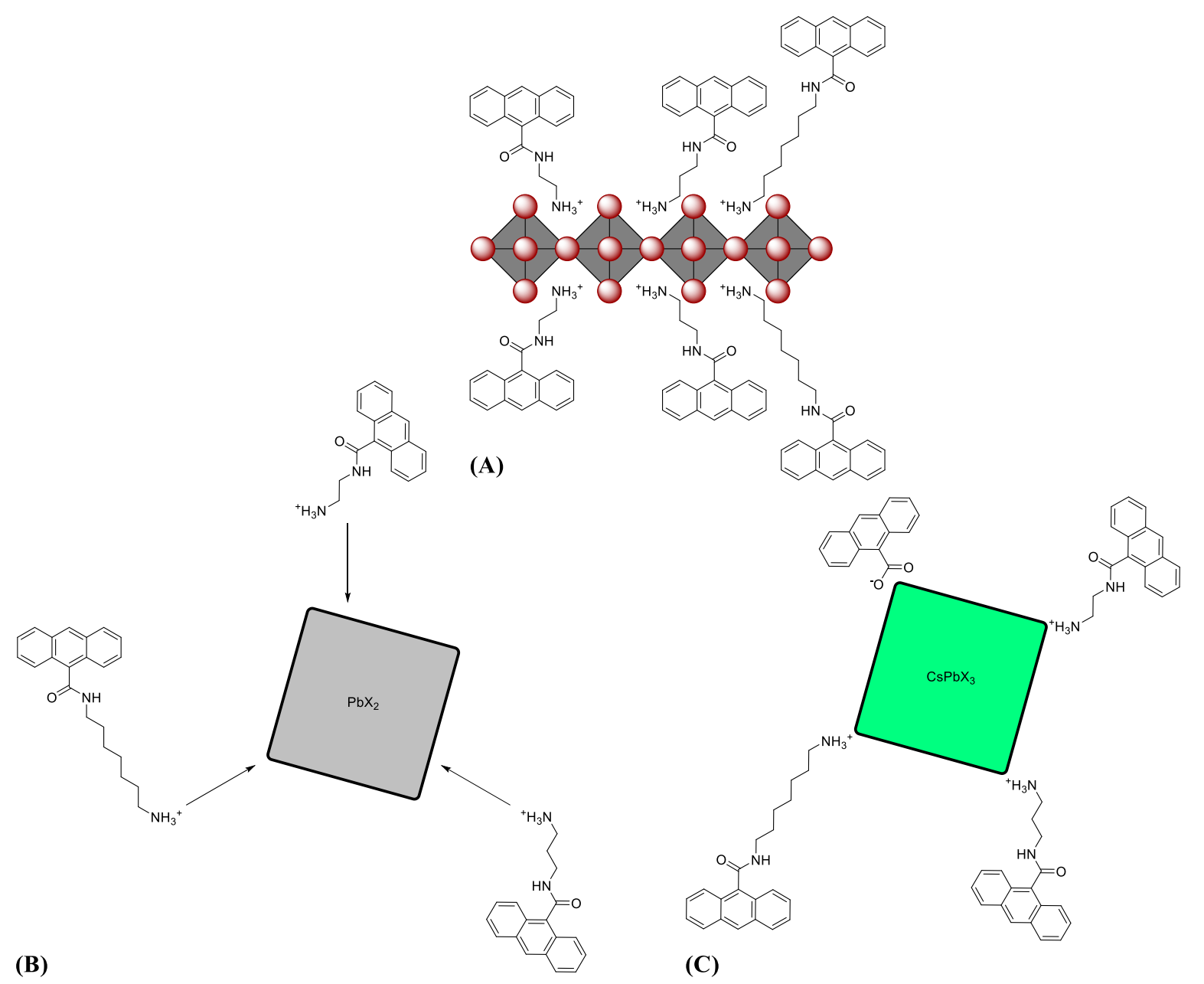

Figure 7a. (A) Schematic summarising a 2D Ruddlesden-Popper Perovskite possess anthracene functionalised A cations. (B) Schematic summarising the intercalation method of converting $P b X_{2}$ nanocrystals into perovskite nanocrystals. (C) Stabilisation of $\mathrm{Cs} P b X_{3}$ nanocrystals with anthracene functionalised ligands.

This master's thesis focused on coupling anthracene derivatives to lead halide perovskites and observing the physical and electronic properties of the resulting materials (see Figure 7a).

Analysis focused on testing whether the anthracene derivatives had been successfully incorporated into the structure of a lead halide perovskite, and determining whether or not there was any form of energy transfer between the organic and inorganic components.

To determine the structure of the perovskites, the following analytical technique was employed:

- Transition electron microscopy 
These methods allowed for the determination of whether the anthracene derivatives had been incorporated into the structure of a perovskite as either a cation within the crystal structure or as a ligand.

The following analytical techniques were employed to determine if there is was energy transfer between the anthracene derivative and the inorganic component of the prepared samples:

- UV-Vis Absorption Spectroscopy

- Emission and Excitation Analysis

- $\quad$ Lifetime analysis

- Photoluminescent Quantum Efficiency (PLQE) 


\section{8. $N$-(Aminoalkyl)Anthracene-9-Carboxamides (AAAC)}

\section{8a. Synthesis of $N$-(Aminoalkyl)Anthracene-9-Carboxamides}

There are three distinct methods that will be attempted to synthesis anthracene functionalised perovskites.

1) Direct thin-film deposition

2) Intercalation of anthracene halide salts into lead halide nanocrystals

3) The stabilisation of caesium lead halide nanocrystals

The anthracene derivatives that were used for perovskite synthesis is a series of $\mathrm{N}$ (aminoalkyl)anthracene-9-carboxamides (AAAC) which possess an amine group at the end of an alkyl chain that extends from a secondary amide functional group. This series is composed of three derivatives, each defined by the length of their alkyl chain. Figure $8 \boldsymbol{a}$ provides a summary of these anthracene derivatives.<smiles>NCCNC(=O)c1c2ccccc2cc2ccccc12</smiles><smiles>NCCCNC(=O)c1c2ccccc2cc2ccccc12</smiles><smiles>CCNC(=O)c1c2ccccc2cc2ccccc12</smiles>

Figure 8a. Series of $\mathrm{N}$-(aminoalkyl)anthracene-9-carboxamides (AAAC). a. $\mathrm{N}$-(2aminoethyl)anthracene-9-carboxamide (AEAC), b. N-(3-aminopropyl)anthracene-9carboxamide (APAC), c. N-(7-aminoheptyl)anthracene-9-carboxamide (AHpAC).

The name of these three derivatives are $N$-(2-aminoethyl)anthracene-9-carboxamide, $N$-(3aminopropyl)anthracene-9-carboxamide, and $N$-(7-aminoheptyl)anthracene-9-carboxamide. Throughout the course of this study the derivatives will commonly be referred to simply as AEAC, APAC, and AHpAC respectively.

Each anthracene derivative was synthesised from the precursor reagent anthracene-9-carboxylic acid (AC) using a two-step methodology utilising an acid chloride intermediate.

\section{Step 1: Synthesis of Anthracene-9-Carbonyl Chloride}

$1 \mathrm{~g}$ of anthracene-9-carboxylic acid was placed into a $25 \mathrm{~mL}$ single neck round bottom flask fitted with a stir bar. $10 \mathrm{~mL}$ of thionyl chloride $\left(\mathrm{SOCl}_{2}\right)$ was then added. A reflux condenser was fitted to the rection vessel and the reaction was stirred at room temperature for two hours. After two hours, the solvent was completely removed via a rotary evaporator fitted with a liquid nitrogen cooled 
condenser. The solid product was then immediately used for the next reaction step (see Figure $8 \boldsymbol{b}$ for reagent details).

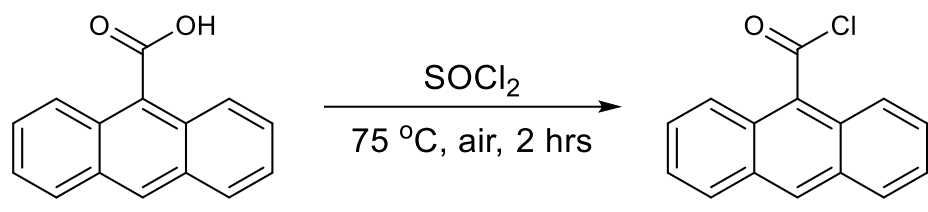

\begin{tabular}{cccccc}
\hline Compound & $\begin{array}{c}\text { Molar } \\
\text { Equivalents }\end{array}$ & mmol & Mass/Volume & $\begin{array}{c}\text { Density } \\
\left(\mathbf{g ~ m L}^{-1}\right)\end{array}$ & $\begin{array}{c}\text { Molar Mass } \\
\left(\mathbf{g ~ m o l}^{-1}\right)\end{array}$ \\
$\begin{array}{c}\text { Anthracene-9- } \\
\text { Carbonyl Chloride } \\
\begin{array}{c}\text { SOCl } \\
\text { SOC }\end{array}\end{array}$ & 1 & 4.49 & $1 \mathrm{~g}$ & - & 222.24 \\
& 137.85 & 137.85 & $10 \mathrm{~mL}$ & 1.64 & 118.97 \\
\hline
\end{tabular}

Figure 8b. Conversion of anthracene-9-carboxylic acid to anthracene-9-carbonyl chloride

\section{Step 2: Formation of (aminoalkyl)anthracene-9-carboxamide}

For a $1 \mathrm{~g}$ scale reaction, $83.25 \mathrm{mmol}$ of diamine $(5.6 \mathrm{~mL}$ of 1,2-diaminoethane, $7.0 \mathrm{~mL}$ of 1,3diaminopropane, or $11.54 \mathrm{~mL}$ of 1,7-diaminoheptane) was added to a $150 \mathrm{~mL}$ round bottom flask and dissolved in $83.25 \mathrm{~mL}$ of dichloromethane (DCM) and cooled to $0{ }^{\circ} \mathrm{C}$ using an ice bath. The synthesised anthracene-9-carbonyl chloride was dissolved within $41.67 \mathrm{~mL}$ (DCM) and then added dropwise to the cool diamine solution. After an hour of stirring, the ice bath was removed, and the reaction allowed to stir overnight.

After a night of stirring, the solvent was removed via rotary evaporation. $10 \mathrm{~mL}$ of water was added to the round bottom. The mixture was sonicated for one hour, after which the solid product was filtered from solvent and dried (see Figure $8 \boldsymbol{c}$ for reagent details) 


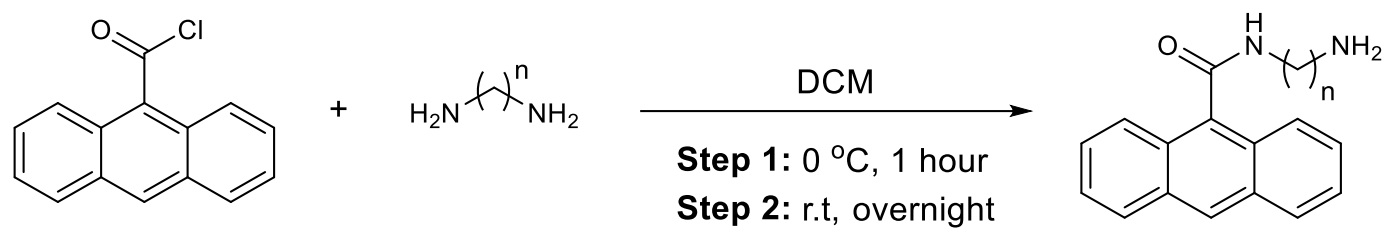

Step 2: r.t, overnight

Compound

Molar

mmol

Mass/Volume

Density

Molar Mass

Equivalents

(g mL $\left.\mathbf{~ L}^{-1}\right)$

(g mol${ }^{-1}$ )

Anthracene-9-

1

4.49

$1.08 \mathrm{~g}$

240.03

Carbonyl Chloride

1,2-diaminoethane

18.5

83.23

$5.56 \mathrm{~mL}$

0.90

60.10

1,3-diaminopropane

18.5

83.23

$7.01 \mathrm{~mL}$

0.88

74.12

1,7-aiaminoheptane

18.5

83.23

$12.61 \mathrm{~mL}$

0.86

130.29

DCM

416.8

375.1

$24.5 \mathrm{~mL}$

1.33

84.93

Figure 8c. Amide formation between anthracene-9-carbonyl chloride and diamine.

\section{8b. Results and Discussion on the Synthesis of $N$-(aminoalkyl)anthracene-9- carboxamide}

\section{Observations}

The addition of anthracene-9-carboxylic acid (AC) to thionyl chloride $\left(\mathrm{SOCl}_{2}\right)$ resulted in a thick, golden suspension. Heating the mixture to $75{ }^{\circ} \mathrm{C}$ resulted in the dissolution of the suspension and the formation of a clear, dark brown solution. After two hours of refluxing the appearance of the solution had not changed. Removal of the solvent left a dark yellow precipitate covering the walls of the flask.

The addition of any diamine to dichloromethane (DCM) produced a clear and colourless solution. The addition of anthracene-9-carbonyl chloride to DCM produced a clear yellow solution. Drop-wise addition of the acid chloride solution to the diamine solution resulted in the development of a white vapour. After the addition of acid chloride was complete, the vapour dissipated and ceased being produced.

For the synthesis of $\mathrm{N}$-(2-aminoethyl)anthracene-9-carboxamide (AEAC) and $\mathrm{N}$-(3aminopropyl)anthracene-9-carboxamide (APAC) overnight stirring of the reaction would produce a white precipitate. Removal of the solvents left a brown oil and a white precipitate. For the synthesis of $\mathrm{N}$-(7-aminoheptyl)anthracene-9-carboxamide, no white precipitate was observed during the course of 
the synthesis and only an oil was collected. The addition of water dissolved any white precipitate present but not the oil. Sonicating or vigorously shaking the mixture resulted in the immediate precipitation of a very pale yellow solid. The isolated solid was pale yellow for all three $\mathrm{N}$ (aminoalkyl)anthracene-9-carboxamides. Reducing and or cooling the water supernatant collected from the synthesis of AEAC and APAC resulted in the re-crystallisation of the white precipitate.

\section{Nuclear Magnetic Resonance Results}

Nuclear magnetic resonance (NMR) analysis of the proton environments present within the filtered products that resulted from the reactions between anthracene-9-carbonyl chloride and the three diamines reveal that the reactions were successful in producing the $N$-aminoalkylanthracene-9carboxamides (see Figures $8 d, 8 e, 8 f, 8 g, 8 h$, and $8 i$ ). 
Anthracene-9-carboxylic acid (AC): ${ }^{1} \mathrm{H} \mathrm{NMR}\left(\mathrm{CDCl}_{3}, 500 \mathrm{MHz}\right): \delta_{\mathrm{H}}, 7.54(2 \mathrm{H}, \mathrm{dd}, J=0.1 \mathrm{~Hz}), 7.61$ $(2 \mathrm{H}, \mathrm{dd}, J=0.1), 8.06(2 \mathrm{H}, \mathrm{d}, J=0.01 \mathrm{~Hz}), 8.33(2 \mathrm{H}, \mathrm{d}, J=0.2 \mathrm{~Hz}), 8.60(1 \mathrm{H}, \mathrm{s})$.

Note: Carboxylic acid protons $(\mathrm{COOH})$ tend to show up as broad singlets a between 13 to $10 \mathrm{ppm}$. No such signal was observed.

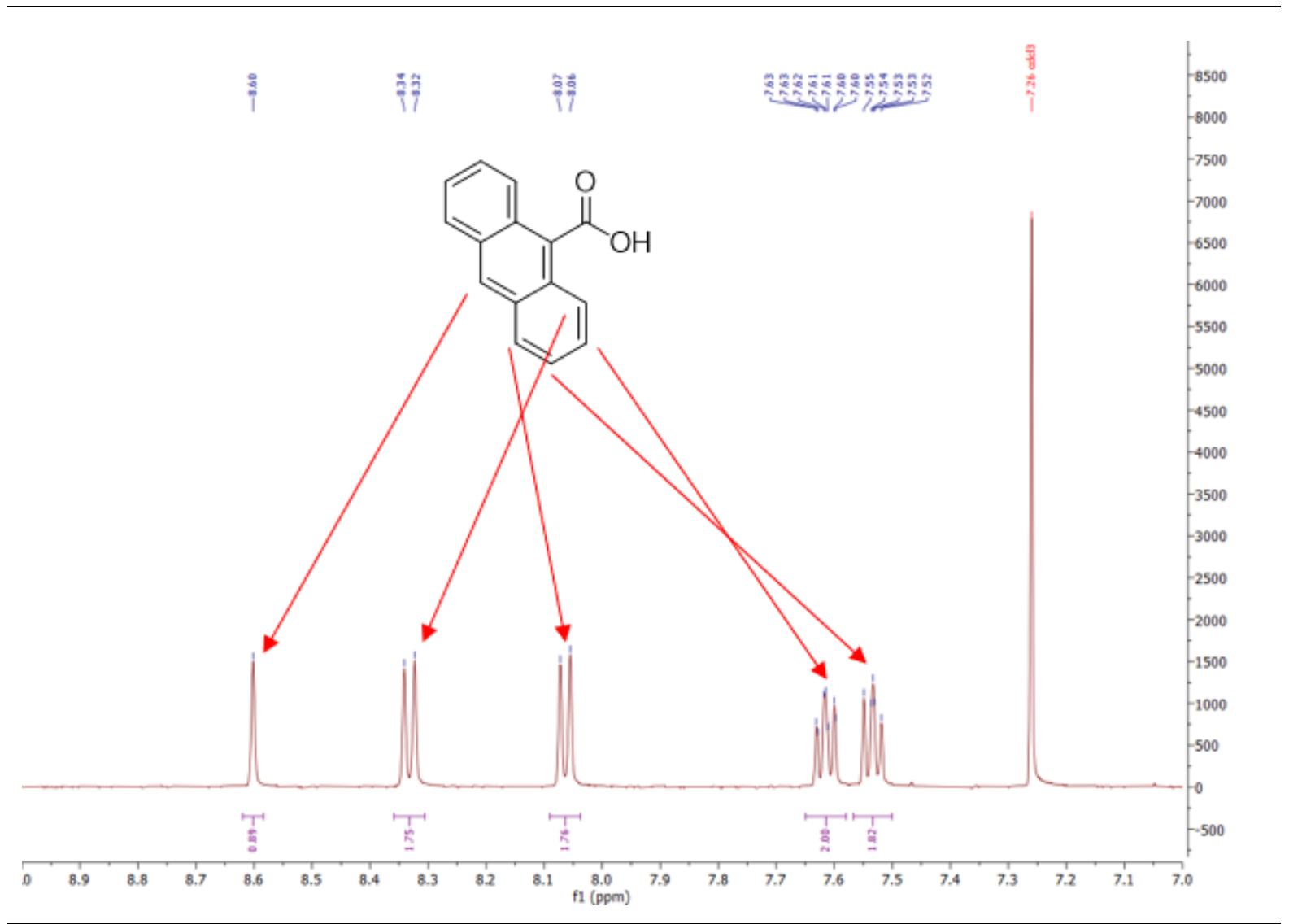

Figure $8 d .{ }^{1} \mathrm{H}$ NMR spectrum of AC, the precursor reagent. 
$N$-(2-aminoethyl)anthracene-9-carboxaimde (AEAC): ${ }^{1} \mathrm{H}$ NMR $\left(\mathrm{CDCl}_{3}, 500 \mathrm{MHz}\right): \delta_{\mathrm{H}}, 3.12(2 \mathrm{H}, \mathrm{p}, J$ $=0.2 \mathrm{~Hz}), 3.80(2 \mathrm{H}, \mathrm{q}, J=0.2), 7.54(4 \mathrm{H}, \mathrm{m}), 8.05(2 \mathrm{H}, \mathrm{d}, J=0.02 \mathrm{~Hz}), 8.12(2 \mathrm{H}, \mathrm{d}, J=0.3 \mathrm{~Hz})$, $8.52(1 \mathrm{H}, \mathrm{s})$.

Note: There are two singlet peaks one at $1.29 \mathrm{ppm}$ and the other at $6.55 \mathrm{ppm}$. These peaks could correspond to the amide proton and terminal amine protons respectively.

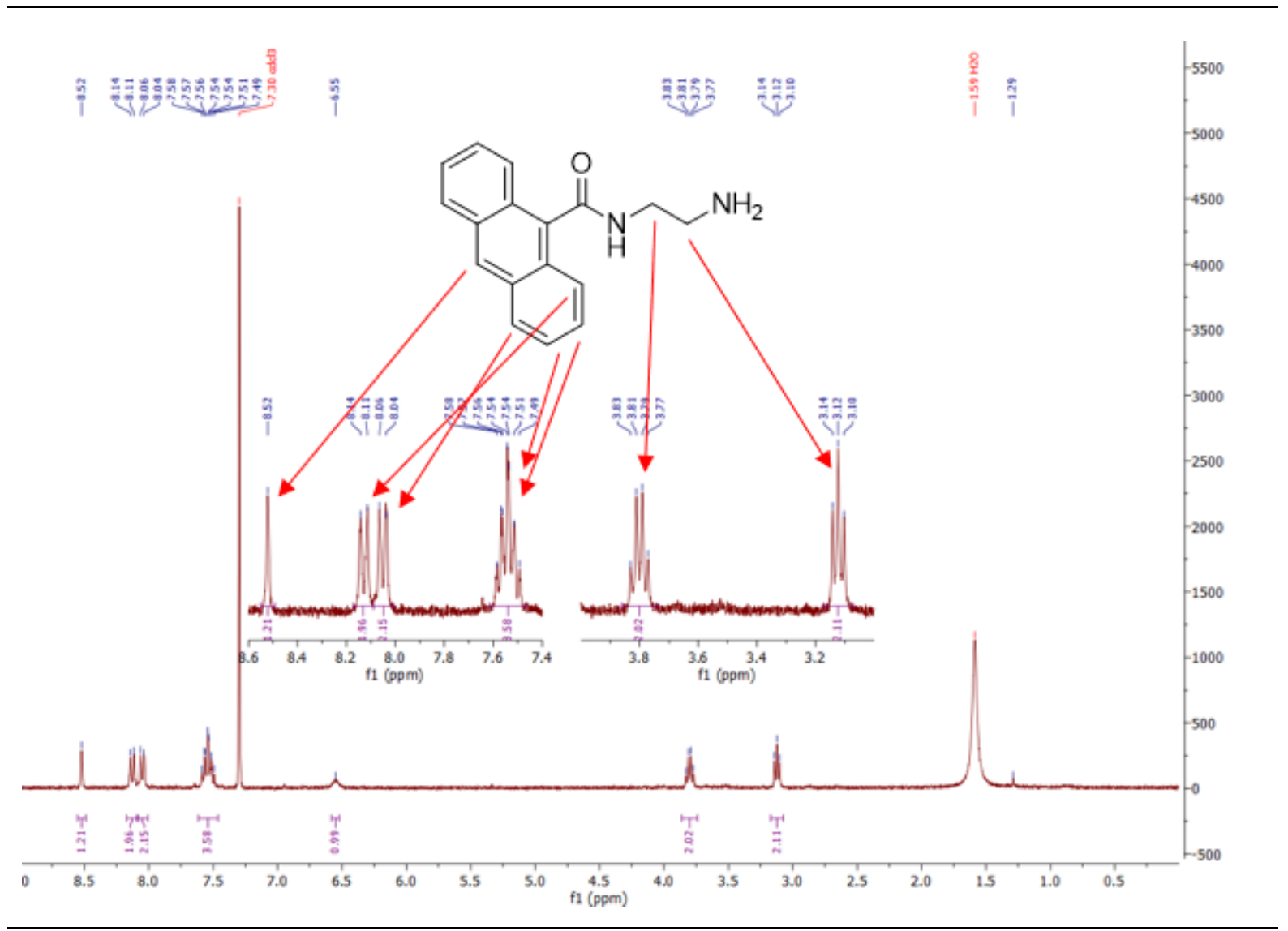

Figure 8e. ${ }^{1} \mathrm{H}$ NMR spectrum of AEAC. 
$N$-(3-aminopropyl)anthracene-9-carboxaimde (APAC): ${ }^{1} \mathrm{H}$ NMR $\left(\mathrm{CDCl}_{3}, 500 \mathrm{MHz}\right): \delta_{\mathrm{H}}, 1.88(2 \mathrm{H}$, m), $2.95(2 \mathrm{H}, \mathrm{m}), 3.69(2 \mathrm{H}, \mathrm{m}), 7.50(4 \mathrm{H}, \mathrm{m}), 8.01(2 \mathrm{H}, \mathrm{d}, J=0.2 \mathrm{~Hz}), 8.07(2 \mathrm{H}, \mathrm{d}, J=0.1 \mathrm{~Hz}), 8.48$ $(1 \mathrm{H}, \mathrm{s})$.

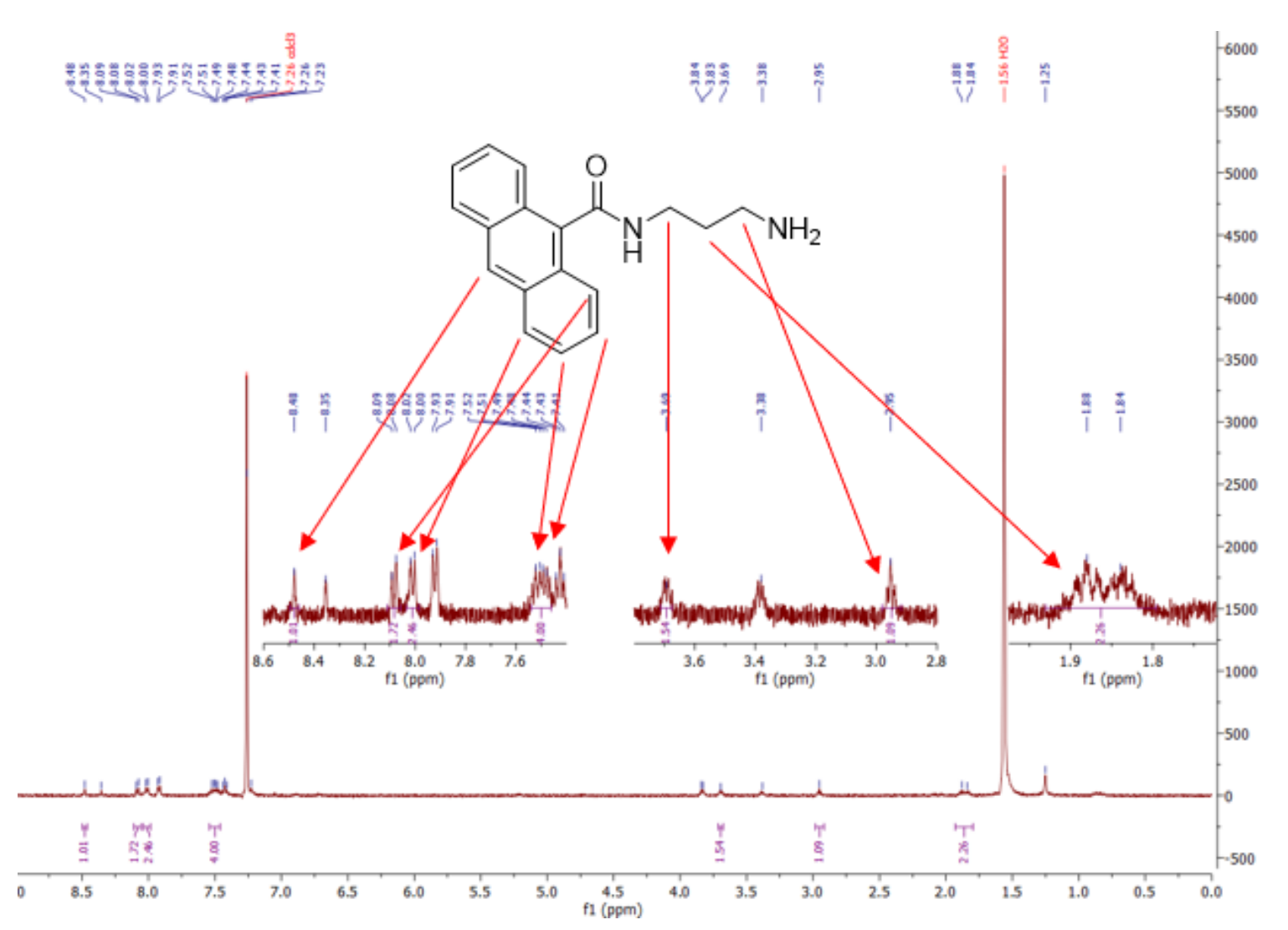

Figure 8f. ${ }^{1} \mathrm{H}$ NMR spectrum of APAC. 
-(7-aminoheptyl)anthracene-9-carboxaimde (AHpAC): ${ }^{1} \mathrm{H}$ NMR $\left(\mathrm{CDCl}_{3}, 500 \mathrm{MHz}\right): \delta_{\mathrm{H}}, 1.64(2 \mathrm{H}$, m), $2.71(2 \mathrm{H}, \mathrm{t}, J=0.2), 3.73(2 \mathrm{H}, \mathrm{q}, J=0.2), 7.54(4 \mathrm{H}, \mathrm{m}), 8.07(4 \mathrm{H}, \mathrm{m}), 8.50(2 \mathrm{H}, \mathrm{s})$.

Note: There are two singlet peaks one at $1.25 \mathrm{ppm}$ and the other at $6.55 \mathrm{ppm}$. These peaks could correspond to the amide proton and terminal amine protons respectively.

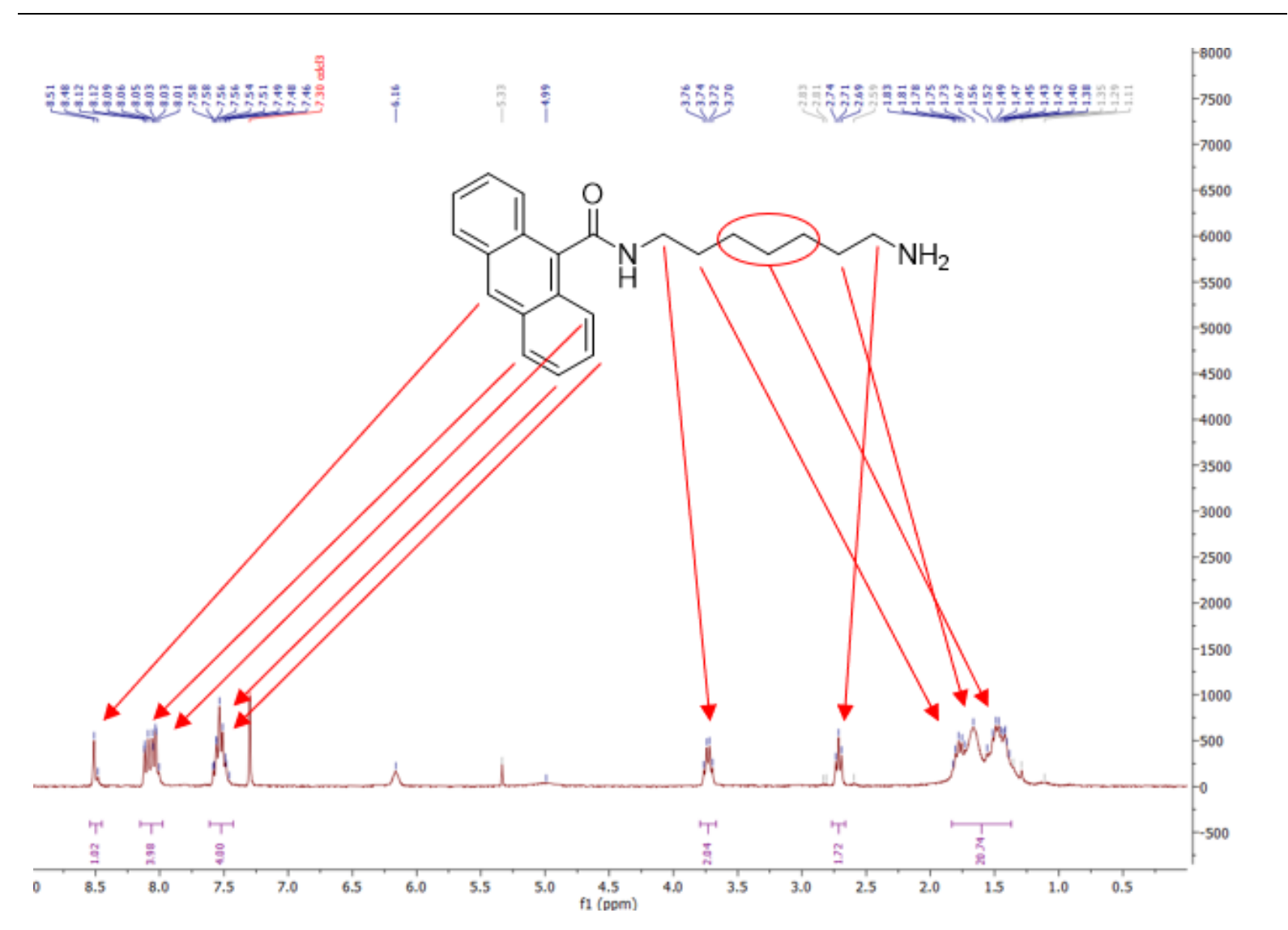

Figure 8g. ${ }^{1} \mathrm{H}$ NMR spectrum of AHpAC. 
The synthesis of both AEAC and APAC tend to produce a second product in the form of a white precipitate. This precipitate is water soluble and can be re-crystallised from the aqueous supernatant after sonicating and filtering the major product. ${ }^{1} \mathrm{H}$ NMR analysis was performed, and the spectrum contains similar peaks to the insoluble product (see Figure $8 d, 8 \boldsymbol{e}$, and $8 f$ ).

2-(anthracene-9-carboxamido)ethan-1-aminium chloride (ACEACl): ${ }^{1} \mathrm{H}$ NMR $\left(\mathrm{D}_{2} \mathrm{O}, 500 \mathrm{MHz}\right): \delta_{\mathrm{H}}$, $3.32(2 \mathrm{H}, \mathrm{t}, J=0.2 \mathrm{~Hz}), 3.83(2 \mathrm{H}, \mathrm{t}, J=0.2), 7.46(2 \mathrm{H}, \mathrm{dd}, J=0.01), 7.50(2 \mathrm{H}, \mathrm{dd}, J=0.01), 7.54$ $(2 \mathrm{H}, \mathrm{d}, J=0.01 \mathrm{~Hz}), 8.03(2 \mathrm{H}, \mathrm{d}, J=0.2 \mathrm{~Hz}), 8.58(1 \mathrm{H}, \mathrm{s})$.

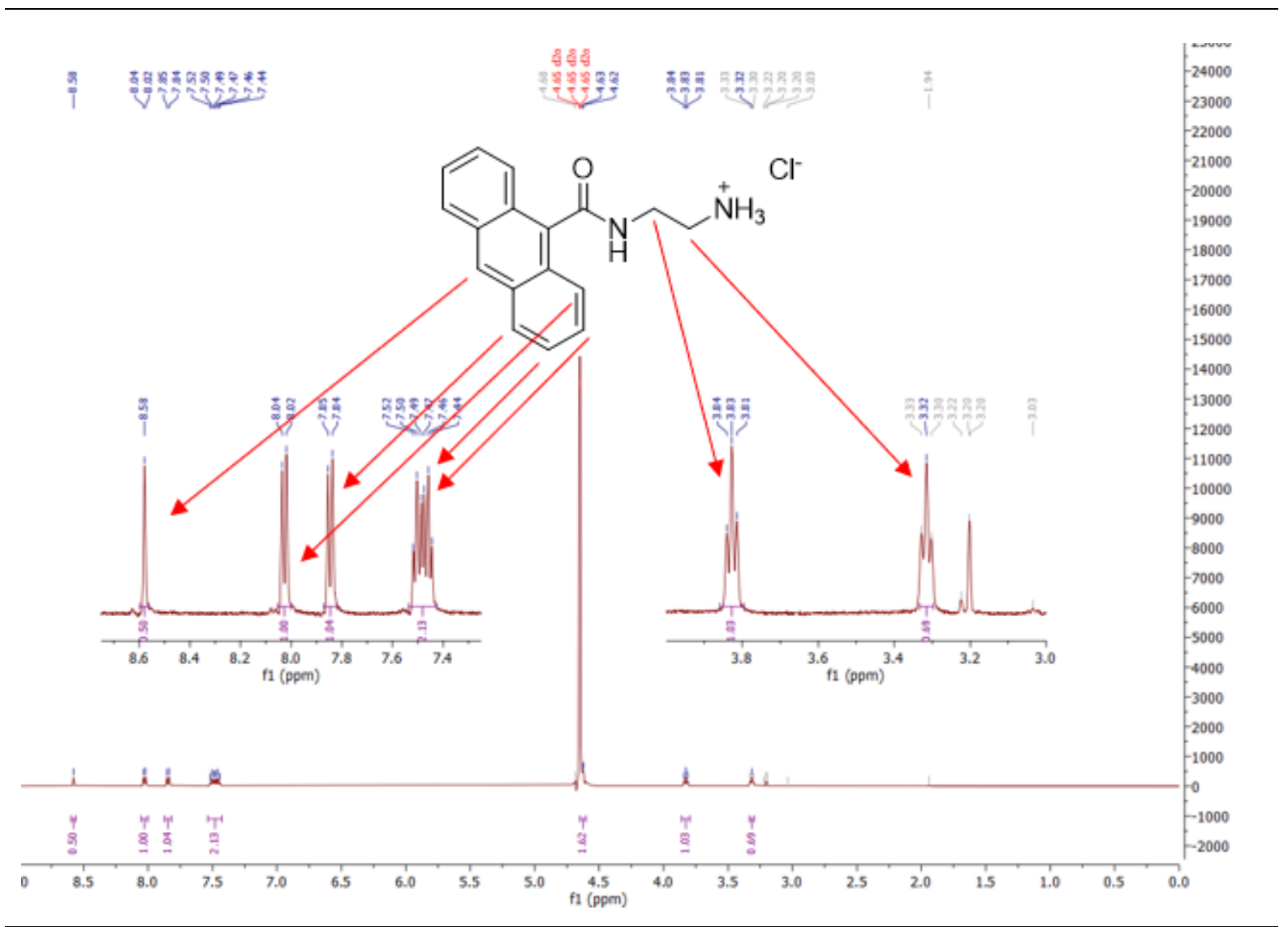

Figure 8h. ${ }^{1} \mathrm{H}$ NMR spectrum of ACEACl. 
3-(anthracene-9-carboxamido)propan-1-aminium chloride (ACPACl): ${ }^{1} \mathrm{H}$ NMR $\left(\mathrm{D}_{2} \mathrm{O}, 500 \mathrm{MHz}\right)$ : $\delta_{\mathrm{H}}$, $1.82(2 \mathrm{H}, \mathrm{p}, J=0.01), 2.75(2 \mathrm{H}, \mathrm{t}, J=0.02), 3.56(2 \mathrm{H}, \mathrm{t}, J=0.01), 7.45(2 \mathrm{H}, \mathrm{dd}, J=0.01), 7.50(2 \mathrm{H}$, $\mathrm{dd}, J=0.01), 8.02(2 \mathrm{H}, \mathrm{d}, J=0.2 \mathrm{~Hz}), 8.07(2 \mathrm{H}, \mathrm{d}, J=0.1 \mathrm{~Hz}), 8.56(1 \mathrm{H}, \mathrm{s})$.

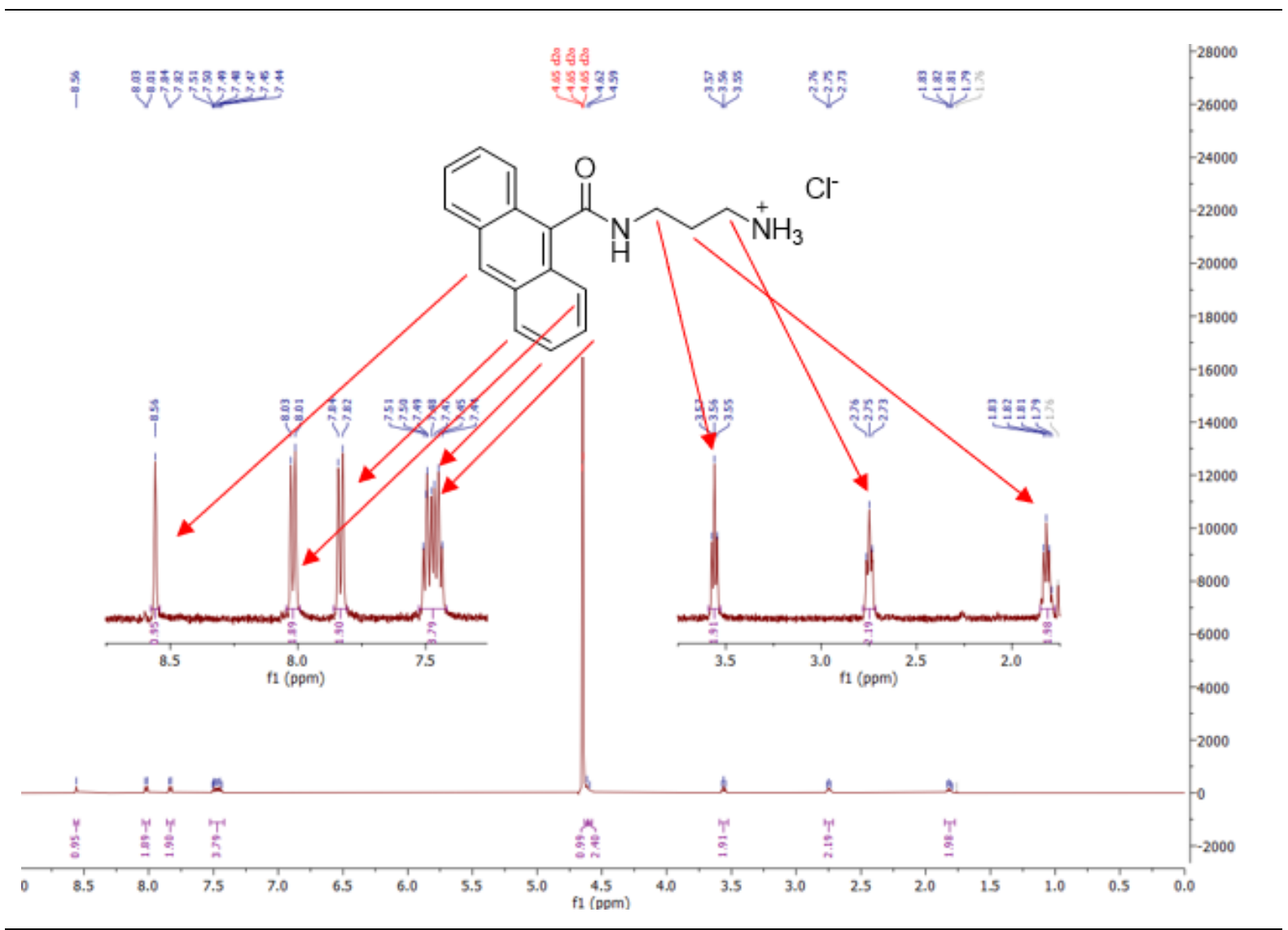

Figure 8i. ${ }^{1} \mathrm{H}$ NMR spectrum of ACPACl.

The water soluble nature of this product coupled with the ${ }^{1} \mathrm{H}$ NMR data suggests that it the protonated form of the neutral amines AEAC and APAC. A by-product of the condensation reaction is an acidic proton. The terminal amines of the neutral products AEAC and APAC can be protonated by this byproduct, resulting in a highly polar, water soluble product that can crystallise out of solution with the other by-product, chloride anions (see Figure $8 j$ ).<smiles>NC(=O)NC(=O)c1c2ccccc2cc2ccccc12</smiles> 


\section{Absorption and Photoluminescence Analysis of Anthracene-9-Carboxylic Acid and $N$ - (Aminoalkyl)Anthracene-9-Carboxamides}

The absorption and photoluminescence properties of solution based anthracene-9-carboxylic acid (AC) was measured. The absorption spectrum displays the characteristic absorption peaks of anthracene, with an absorption onset at $\sim 400 \mathrm{~nm}$ and a rapid decay in absorption intensity at wavelengths shorter than $350 \mathrm{~nm}$. The photoluminescence of the sample indicates excimer formation, as the peak emission wavelength is at $\sim 460 \mathrm{~nm}$, while anthracene monomer emission tends to display a peak intensity of $\sim 410 \mathrm{~nm}$. The excitation spectrum of the sample correlates perfectly with the absorption spectrum, indicating low levels of self-quenching (see Figure $8 \boldsymbol{k}$ ).

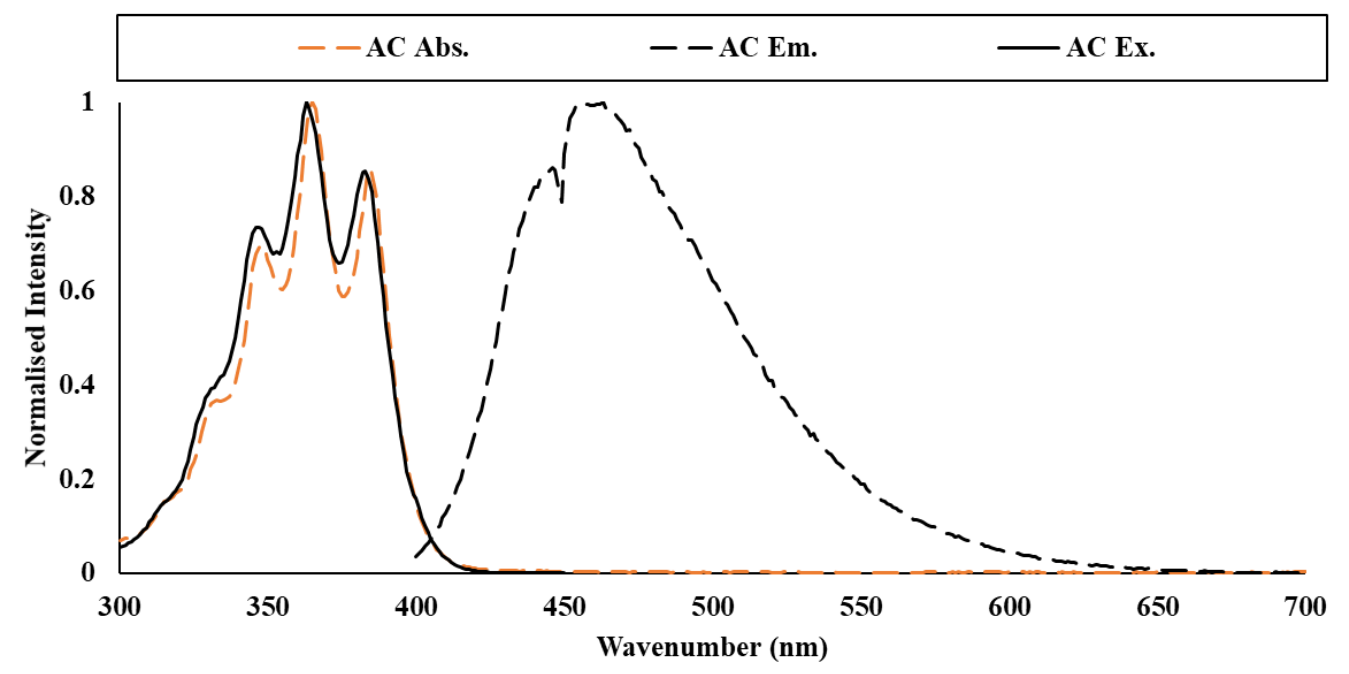

Figure 8k. (A) Overlay of the absorption and photoluminescence spectra of anthracene-9carboxylic acid. (B) Emission lifetime of anthracene-9-carboxylic acid.

The absorption and photoluminescence of the three $N$-(aminoalkyl)anthracene-9-carboxamides (AAAC) was analysed for both thin-film and solution based samples (see Figure $\mathbf{8} \boldsymbol{m}$ and $8 \boldsymbol{n}$ ). All three thin-film based samples demonstrate practically the same absorption spectrum, with an absorption onset at $400 \mathrm{~nm}$, four prominent peaks and one shoulder peak. A thin-film of pure AC was not measured as no experiment was performed requiring a thin-film AC standard to compare to.

While the absorption spectra are highly similar, there is significant variation among the emission and excitation spectra. Low concentration samples of anthracene display peak emission at 400 to $410 \mathrm{~nm}$. However, all three AAAC thin-films display strongly redshifted and broadened emission signals, indicative of excimer formation and emission. There is a clear relationship between excimer formation and the length of the alkyl chain, with the emission spectrum of $\mathrm{N}$-(2aminoethyl)anthracene-9-carboxamide (AEAC) dominated by excimer formation and $\mathrm{N}$-(7aminoheptyl)anthracene-9-carboxamide (AHpAC) displaying both monomer emission at $414 \mathrm{~nm}$ and 
excimer emission at $520 \mathrm{~nm}$. From these results it is clear that increasing the length of the alkyl chain acts to force apart the anthracene functional groups and reduce/hinder excimer formation.

The excitation spectra of the thin-films also display variance. AEAC displays a narrow excitation range of between 400 and $300 \mathrm{~nm}$. This range displays three small peaks that correlate positively with the absorption spectrum, indicating that the concentration of anthracene functional groups within the sample is not high enough to prevent peak absorption wavelengths from travelling through and exciting the bulk of the sample. However, both $N$-(3-aminopropyl)anthracene-9-carboxamide (APAC) and $\mathrm{AHpAC}$ display a negative correlation between there electronic excitation and absorption spectra, indicating that the concentration of these samples was high enough to prevent bulk excitation of the sample by the peak absorption wavelengths. Additionally, as the length of the alkyl chain increases, the intensity of excitation that results from wavelengths between 350 and $300 \mathrm{~nm}$ increases significantly. Therefore, increased excitation due to high energy wavelengths is associated with increased monomer emission.

Absorption measurements of the AAAC in toluene solutions show some results which mirror those of the thin-films, and others that are quite unique (see Figure $8 \boldsymbol{n}$ ). The absorption spectra of APAC and $\mathrm{AHpAC}$ is almost identical to that of the thin-film equivalents. Solution based AEAC demonstrates a unique absorption spectrum, namely an additional absorption peak at $\sim 405 \mathrm{~nm}$, and a high intensity, dual peaked absorption band between 300 and $350 \mathrm{~nm}$. Though the additional peak at $\sim 405 \mathrm{~nm}$ does correlate with the excitation spectrum, the high energy absorption band does not result in heightened excitation. Therefore, this increased absorption feature must be attributed to a process that does not result in fluorescence. One possibility is the photodimerization of anthracene, which is a [4+4] cycloaddition reaction that is induced by irradiation of wavelengths less than $350 \mathrm{~nm}$ (see Figure $8 \boldsymbol{l}$ ). This explanation works for two reasons. Firstly, the intensity of this absorption band drops significantly after $350 \mathrm{~nm}$ and according to Laurent et al, anthracene photodimers do not absorb at wavelengths less than $300 \mathrm{~nm}$ due to the reduced conjugation of the system. ${ }^{63}$ Secondly, the photo dimer does not fluoresce, explaining the negative correlation between absorption and excitation in this region of the spectrum.

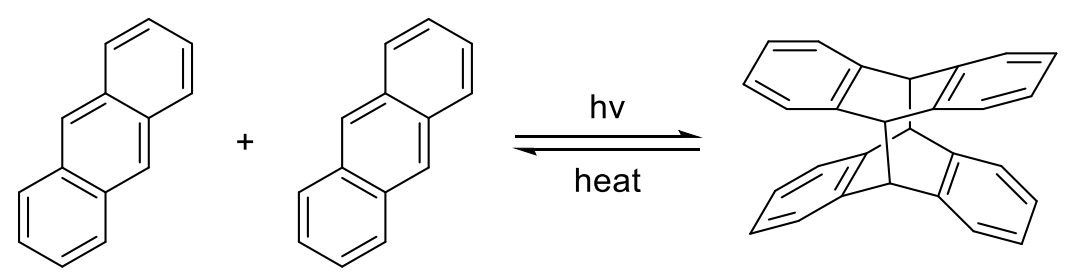

Figure 8l. [4+4] Cycloaddition of two anthracene monomers to form an anthracene dimer. 
The emission of all three samples is dominated by monomer emission, indicating that excimer emission was not favourable in the measured samples, likely to be a result of the low concentration of the sample which prevents anthracene functional groups from coming into close enough proximity for excimer formation. Another result of the samples low concentrations is the positive correlation between electronic excitation and emission and therefore lack of self-quenching. 

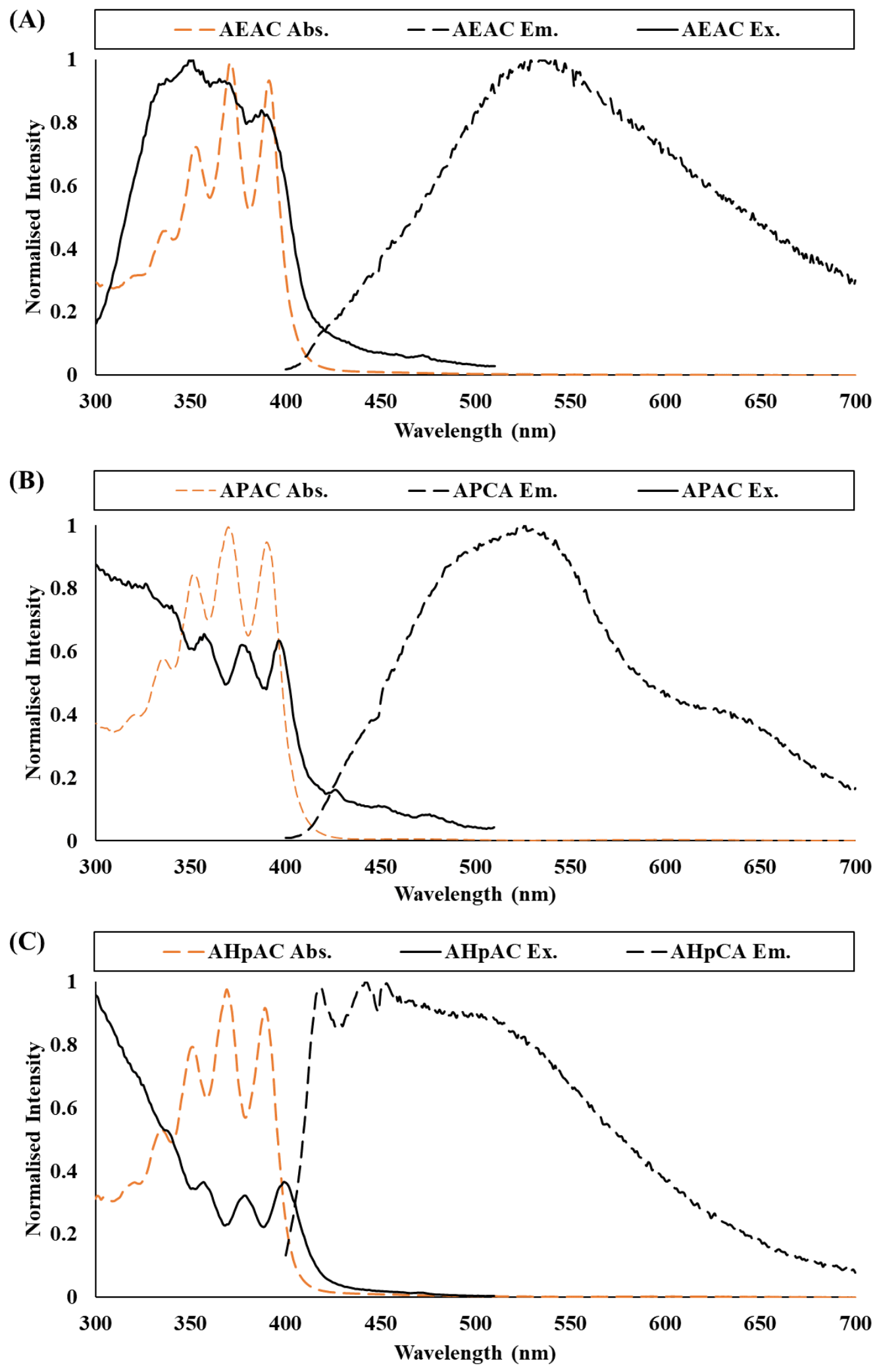

Figure 8m. (A) Overlay of the absorption, electronic emission and excitation of pure thin-films of $A E A C(A), A P A C(B)$, and $A H p A C(C)$. 
(A)

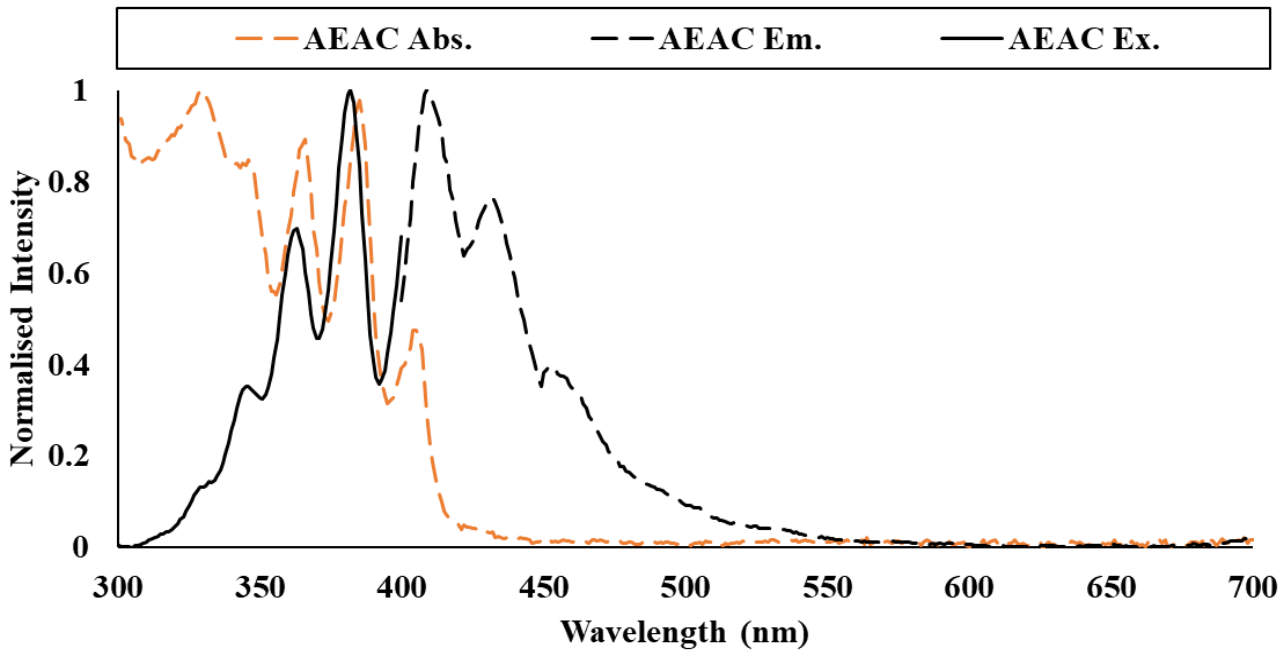

(B)

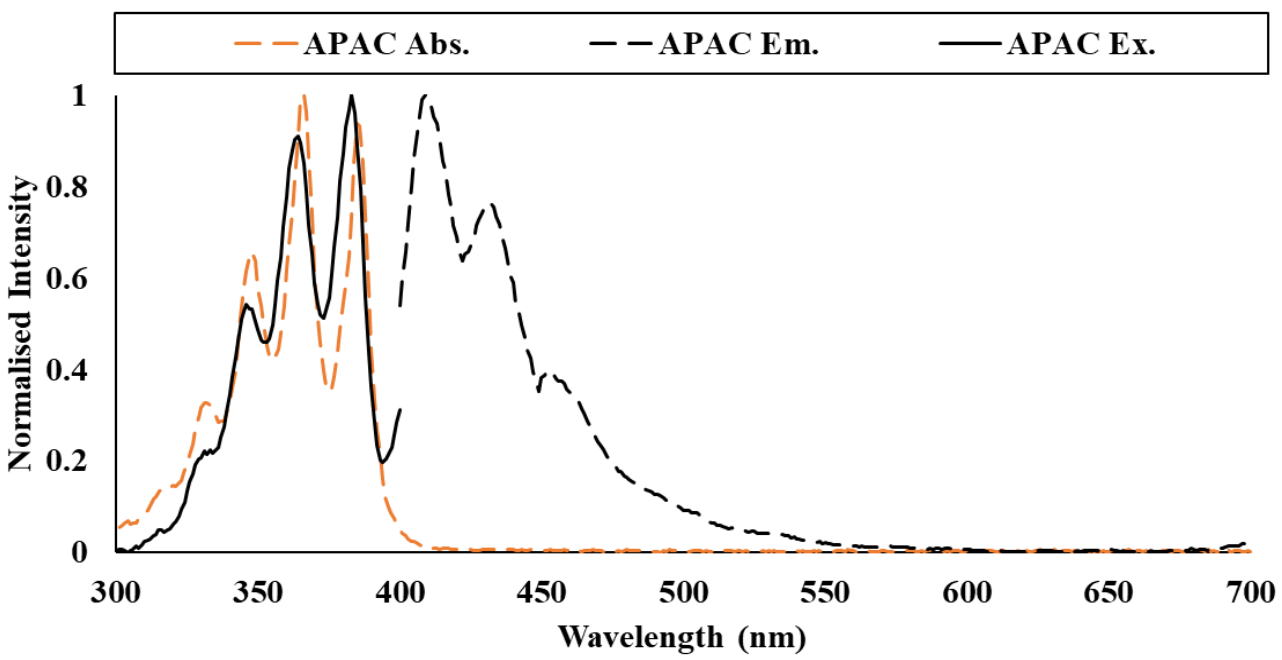

(C)

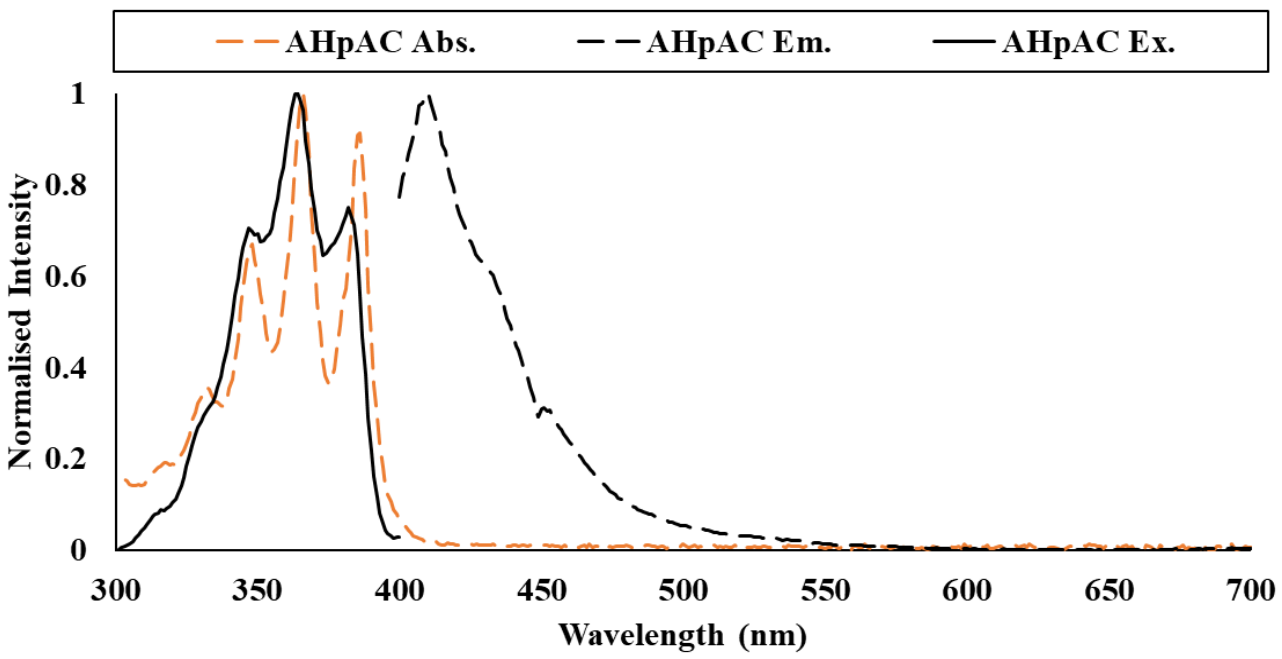

Figure 8n. (A) Overlay of the absorption, electronic emission and excitation of pure toluene solutions of $A E A C(A)$, pure $A P A C(B)$, and pure $A H p A C(C)$. 
Emission lifetime measurements were performed on AC and all six AAAC samples. The solution based samples demonstrate a clear trend between the size of the anthracene substituent and the rate of emission decay. Specifically, AC possess the smallest substituent which is a carboxylic acid functional group and demonstrates the longest lifetime while AHpAC possesses the most spatially demanding substituent and demonstrates the shortest emission lifetime.

While all of the solution based samples demonstrated comparable emission lifetimes, the thin-film sample AEAC displays a dramatically shorter emission lifetime when compared to the other thin-film samples. This result may be coupled with the excimer dominant emission of the signal, and the longer emission lifetimes of the other two thin-film samples is due to monomer emission (see Figure 8o).
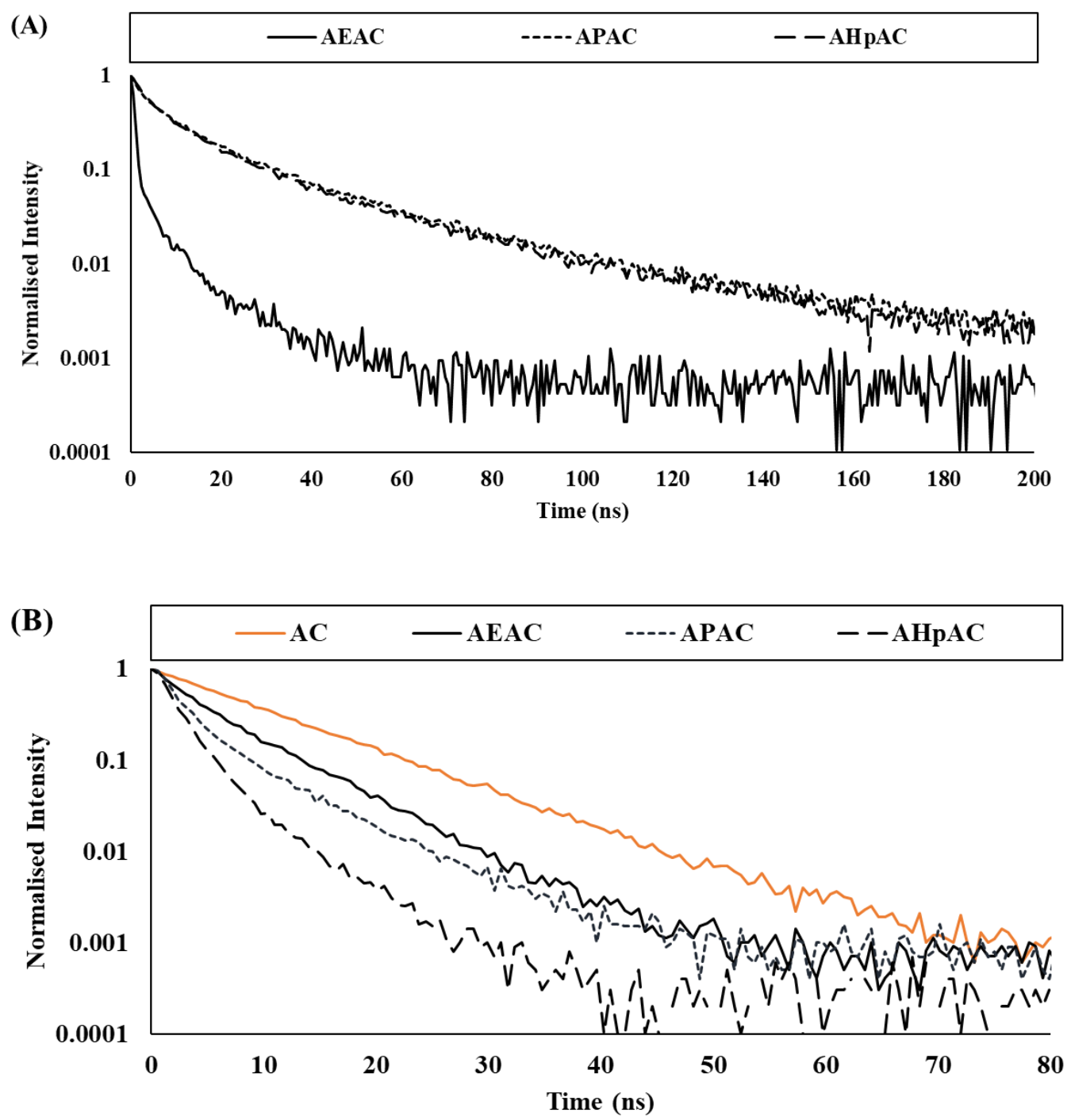

Figure 8o. Comparison between the emission lifetimes of AEAC, APAC, and AHpAC thin-films (A) and toluene solutions (B). 


\section{8c. Concluding Remarks on the Synthesis of $N$-(aminoalkylanthracene-9-Carboxamides}

The synthesis of all three $N$-(aminoalkyl)anthracene-9-carboxamides was successful as suggested by the ${ }^{1} \mathrm{H}$ NMR data collected. Despite the simplicity of the synthesis, the NMR data indicated surprisingly clean samples for 4 out 5 of the collected products. While the NMR spectrum of $\mathrm{N}$-(3aminopropyl)anthracene-9-carboxamide (APAC) indicted a highly impure product, the isolated salt 3(anthracene-9-carboxamido)propan-1-aminium chloride (ACPACl) was highly pure. Since APAC samples will need to be converted into a series of 3-(anthracene-9-carboxamido)propan-1-aminium halide (ACPAX) salts, this will provide an opportunity to purify the product.

The absorption and photoluminescence data of the products were analysed, indicating that in solution, the presence of an alkyl chain does not greatly influence the fluorescence properties of the anthracene functional group. However, while in the solid state, the length of the alkyl chain does influence the degree of excimer formation and emission. 


\section{9. (Anthracene-9-Carboxamido)Alkylaminium Lead Halide}

\section{([ACAA $\left.]_{2} \mathbf{P b X}_{4}\right)$ Thin-Films}

\section{9a. Synthesis of (ACAA) $)_{2} \mathrm{PbX}_{4}$ Thin-Films}

The aim of this project was to attempt to synthesis a 2D Ruddlesden-Popper perovskite thin-film which possesses one of the three protonated $N$-(alkylaminium)anthracene-9-carboxamides (AAAC) as an A cation for the perovskite. The synthesis method required the neutral AAAC to be converted into an (anthracene-9-carboxamido)alkylaminium halide (ACAAX) salt via protonation by a hydrogen halide acid solution (see Figure 9a). Fortunately, the synthesis of $N$-(2-aminoethyl)anthracene-9carboxamide (AEAC) and $N$-(3-aminopropyl)anthracene-9-carboxamide (APAC) produces hydrogen chloride $(\mathrm{HCl})$ as a by-product which acts to partially convert some of the major product into the equivalent chloride salts $\mathrm{ACEACl}$ and $\mathrm{ACPACl}$. While the synthesis of $N$-(7-aminoheptyl)anthracene9-carboxamide also produces $\mathrm{HCl}$, no significant quantity of $\mathrm{ACHpACl}$ could be extracted and therefore has to be made via a separate step.

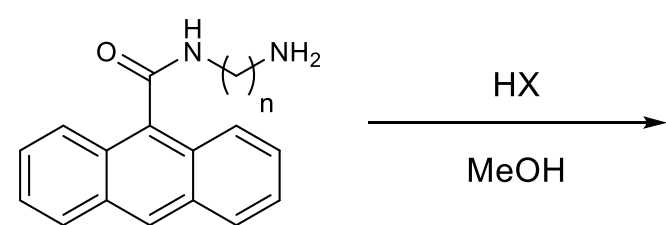

AAAC<smiles>NC(N)C(=O)c1c2ccccc2cc2ccccc12</smiles>

ACAAX

Figure 9a. The protonation of an (aminoalkyl)anthracene-9-carboxamide (AAAC) by a hydrogen halide (HX) to form an (anthracene-9-carboxamido)alkylaminium halogen salt (ACAAX).

The conversion of a neutral AAAC into an ACAAX salt was done by dissolving the neutral species in the minimum amount of hot methanol, followed by the addition of one to two molar equivalents of hydrogen halide and leaving the mixture to stir for 1 hour. For AEAC and APAC, cooling the reaction mixture is sufficient to induce crystallisation of product. For AHpAC, the product salt would not crystallise out from solution and therefore the solvent had to be removed via rotary evaporation and the crude product re-crystallised using a minimum amount of hot methanol.

The ACAAX salt was then combined with a lead (II) halide in a 2.5:1 ratio to give a total mass of 26 $\mathrm{mg}$ of solid reagents. ${ }^{64}$ This solid mixture was dissolved in $2.5 \mathrm{~mL}$ dimethylformaldehyde (DMF) and stirred at $120{ }^{\circ} \mathrm{C}$ for 1 hour (see Figure $9 \boldsymbol{b}$ ). A drop of this solution was then deposited onto a heated glass substrate to allow the DMF to vaporise and the reagents to precipitate to form a solid film. 


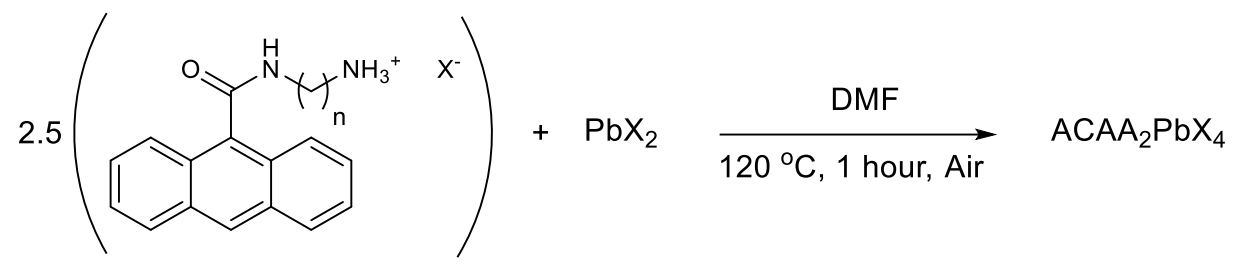

Figure 9b. The chemical equation for the formation of the 2D Ruddlesden-Popper perovskite $(\mathrm{ACAA})_{2} \mathrm{PbX}_{4}$.

\section{$\underline{\text { Analysis }}$}

All samples had their absorption spectrum measured on a Varian Cary 50 Scan UV/Vis Spectrophotometer, their photoluminescence properties including emission, excitation, photoluminescence quantum yield, and emission lifetimes were measured on an Edinburgh Instruments FLS980 photoluminescence spectrometer. Note: While X-Ray Diffraction data was desired, the necessary equipment was unavailable during the duration of this project.

\section{9b. (ACAA) $)_{2} \mathrm{PbX}_{4}$ Thin-Film Results and Discussion}

\section{Observations}

Dissolution of the neutral AAAC in hot methanol results in a clear and pale yellow solution. AEAC and APAC readily dissolve, while AHpAC requires significantly more solvent. The addition of an HX acid solution results in the production of a small amount of white vapour. For AEAC and APAC, as long as a high temperature is maintained, no precipitation will occur. As soon as the mixture is allowed to cool, precipitation commences. For AHpAC, even cooling to below $0{ }^{\circ} \mathrm{C}$ does not induce precipitation. Removal of the solvent via evaporation results in a solid remnant. Redissolution of this solid in a minimum amount of hot methanol and subsequent cooling induces crystallisation.

All solutions of ACAAX and lead halide $\left(\mathrm{PbX}_{2}\right)$, where $\mathrm{X}=\mathrm{Cl}$ or $\mathrm{Br}$, are slightly yellow in colour. Stirring at $120^{\circ} \mathrm{C}$ for one hour does not change the appearance of the solution. Depositing of this solution via either drop casting or spin-coating results in an almost colourless, clear, glassy solution. All iodide based solutions were strongly coloured yellow and heating the solution for an hour does not change the appearance. Deposition of this solution onto a glass slide forms a yellow film that is clear and glassy in appearance.

All three chloride samples demonstrated observable fluorescence, $\mathrm{PbBr}_{2}+\mathrm{ACEABr}$ and $\mathrm{PbBr}_{2}+\mathrm{ACPABr}$ both noticeably fluoresced while $\mathrm{PbBr}_{2}+\mathrm{ACHpABr}$ did not display obvious fluorescence. Both $\mathrm{PbI}_{2}+\mathrm{ACEAI}$ and $\mathrm{PbI}_{2}+\mathrm{ACPAI}$ weakly fluoresced while $\mathrm{PbI}_{2}+\mathrm{ACHpAI}$ did not 
fluoresce (Figures $\mathbf{9 f}$, $9 \boldsymbol{g}$, and $\mathbf{9 h}$ provide a more quantitative description of these samples fluorescent properties.)

\section{Absorption Measurements of (Anthracene-9-Carboxamido)Alkylaminium Lead Halide Thin- Films}

The absorption of the mixed $\mathrm{PbX}_{2}+\mathrm{ACAAX}$ films strongly matches that of the pure $\mathrm{N}$ (alkylaminium)anthracene-9-carboxamides (AAACs).

For the chloride samples, the lack of absorption by pure lead chloride $\left(\mathrm{PbCl}_{2}\right)$ is reflected in the samples $\mathrm{ACPA}_{2} \mathrm{PbCl}_{4}$ and $\mathrm{ACHpA}_{2} \mathrm{PbCl}_{4}$, in which the absorption spectra of these samples strongly correlate with the respective AAAC sample. Overlaying the absorption spectra of the pure AAAC films and that of the pure $\mathrm{PbCl}_{2}$ films results in an absorption pattern that strongly matches that of the mixed films (see Figure 9c).

However, for the sample $\mathrm{PbCl}_{2}+\mathrm{ACEACl}$, between 300 and $350 \mathrm{~nm}$, the samples absorption spectrum deviates significantly from both $\mathrm{AEAC}$ and $\mathrm{PbCl}_{2}$, suggesting an entirely new feature that results from an interaction between $\mathrm{PbCl}_{2}$ and $\mathrm{ACEACl}$. The absorption feature is a sharp peak at 332 $\mathrm{nm}$. In addition, the absorption of this sample between 300 and $330 \mathrm{~nm}$ is significantly greater than that of either the pure $\mathrm{AEAC}$ film and $\mathrm{PbCl}_{2}$. One explanation behind this absorption feature is the presence of a perovskite phase. According to Papvasiliou et al the excitonic absorption peak for a two dimensional lead chloride perovskite arises at around $332 \mathrm{~nm}$ and at wavelengths that are shorter than this the absorption steadily increases. While the scanning range for this sample only extends to 300 $\mathrm{nm}$ and therefore no definitive increase in absorption can be observed, the correlation of the peak plus the absorption at range suggests a new electronic transition.

However, an alternative explanation is the formation of an excimer or a dimer between adjacent anthracene functional groups. Such an interaction could be induced by the presence of the lead halide, distorting how the ACEA molecules would pack under normal conditions, and therefore allow for new interactions between neighbouring anthracene functional groups. In addition, the increased absorption between 300 and $350 \mathrm{~nm}$ could simply be a result of light scattering and not due to a perovskite phase.

Besides this low wavelength region, the absorption spectrum is practically identical between the lead chloride containing films and the lead free, pure AAAC films. 

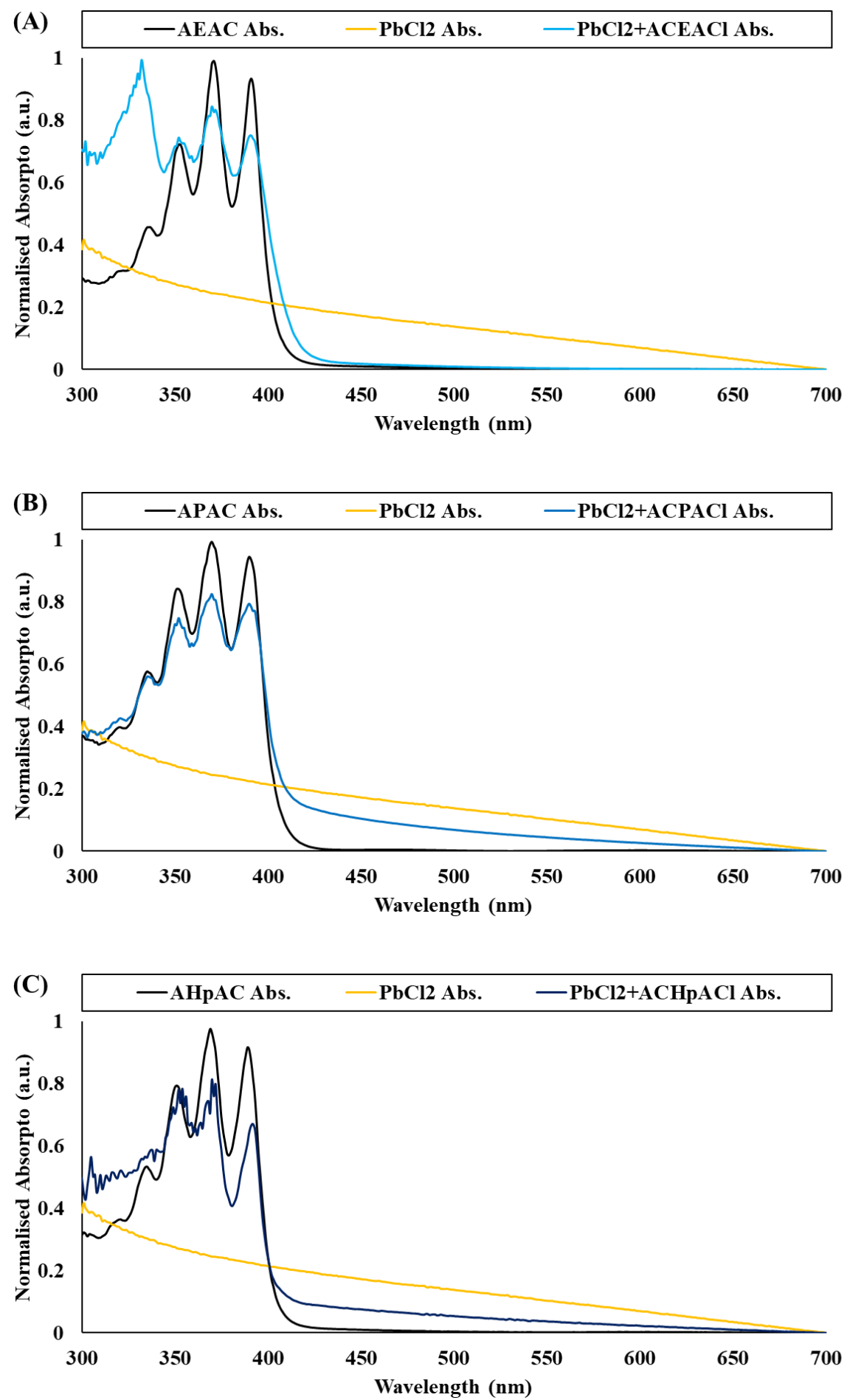

Figure 9c: (A) Comparison between the absorption of pure films of $\mathrm{AEAC}$ and $\mathrm{PbCl}_{2}$ with that of a film of $\mathrm{PbCl}_{2}+\mathrm{ACEACl}$. (B) Comparison between the absorption of pure films of APAC and $\mathrm{PbCl}_{2}$ with that of a film of $\mathrm{PbCl}_{2}+\mathrm{ACPACl}$. (B) Comparison between the absorption of pure films of $\mathrm{AHpAC}$ and $\mathrm{PbCl}_{2}$ with that of a film of $\mathrm{PbCl}_{2}+\mathrm{ACHpACl}$.

Like the previously discussed chloride series, the bromide series of samples display absorption spectra that is dominated by anthracene (see Figure 9d). However, both $\mathrm{PbBr}_{2}+\mathrm{ACEABr}$ and 
$\mathrm{PbBr}_{2}+\mathrm{ACPABr}$ display a large, broad absorption feature between 345 and $300 \mathrm{~nm}$. As discussed previously, the chloride sample $\mathrm{PbCl}_{2}+\mathrm{ACEACl}$ also demonstrates an absorption band in a similar region of the spectrum. However, the shapes of the absorption bands demonstrated by these two bromide samples is distinct from that of the chloride. While the feature displayed by $\mathrm{PbCl}_{2}+\mathrm{ACEACl}$ is a single band with a sharp peak, the short wavelength absorption band displayed by both bromide samples is broad and has two peaks that align well with the two higher energy absorption peaks of the AAAC films at $\sim 320 \mathrm{~nm}$ and $\sim 335 \mathrm{~nm}$, however the relative intensity of these peaks is much greater in these two lead bromide containing samples. One explanation for this increase in absorption is the presence of lead bromide $\left(\mathrm{PbBr}_{2}\right)$. Thin-films of $\mathrm{PbBr}_{2}$ significantly absorbs at wavelengths shorter than $360 \mathrm{~nm}$. Such an absorption pattern correlates well with the broad absorption features of $\mathrm{PbBr}_{2}+\mathrm{ACEABr}$, suggesting that this feature is a combination of the absorptions of the AAAC molecule and $\mathrm{PbBr}_{2}$.

An issue with this hypothesis includes the sudden drop in absorption at wavelengths shorter than 320 $\mathrm{nm}$, since $\mathrm{PbBr}_{2}$ films consistently absorbs beyond this wavelength down to $300 \mathrm{~nm}$. In addition, $\mathrm{PbBr}_{2}+\mathrm{ACHpABr}$ does not possess a broad increase in absorption and instead displays a constant absorption rate between $300 \mathrm{~nm}$ and $\sim 330 \mathrm{~nm}$. Such an absorption spectrum correlates extremely well with an overlay of the absorption of $\mathrm{AHpAC}$ and $\mathrm{PbBr}_{2}$, suggesting that while the absorption of $\mathrm{PbBr}_{2}$ cannot explain the absorption spectra of $\mathrm{PbBr}_{2}+\mathrm{ACEABr}$ and $\mathrm{PbBr}_{2}+\mathrm{ACPABr}$, it does suggest that the sample $\mathrm{PbBr}_{2}+\mathrm{ACHpABr}$ is simply composed of separate phases of $\mathrm{ACHpABr}$ and $\mathrm{PbBr}_{2}$ that absorb independently of each other.

Referring back to the high energy absorption feature of $\mathrm{PbBr}_{2}+\mathrm{ACEABr}$ and $\mathrm{PbBr}_{2}+\mathrm{ACPABr}$, this interaction cannot be attributed to a perovskite phase, as it has been reported that the typical absorption range of two-dimensional lead bromide perovskites is around $400 \mathrm{~nm} .{ }^{65,66,67} \mathrm{In}$ addition, the absorption spectra of layered perovskites tends to increase with decreasing wavelength right up to $200 \mathrm{~nm}$. Remembering again that the broad absorption band of $\mathrm{PbBr}_{2}+\mathrm{ACEABr}$ and $\mathrm{PbBr}_{2}+\mathrm{ACPABr}$ drops off at wavelengths shorter than $320 \mathrm{~nm}$, further reducing the likelihood that these absorption features are the result of a semiconducting perovskite phase. In addition, if a perovskite phase was present, the first absorption peak of anthracene at $400 \mathrm{~nm}$ would be distorted due to the presence of the excitonic absorption peak. The lack of this distortion also supports that there is no perovskite phase.

An alternative explanation for the broad absorption band is that it is a result of the ACAABr itself. The broad absorption band at 320 to $360 \mathrm{~nm}$ strongly resembles a short wavelength absorption feature displayed by solution based AEAC. That feature shared a negative correlation with the electronic excitation spectrum of that sample, and the intensity of the peak drop significantly after $350 \mathrm{~nm}$. For 

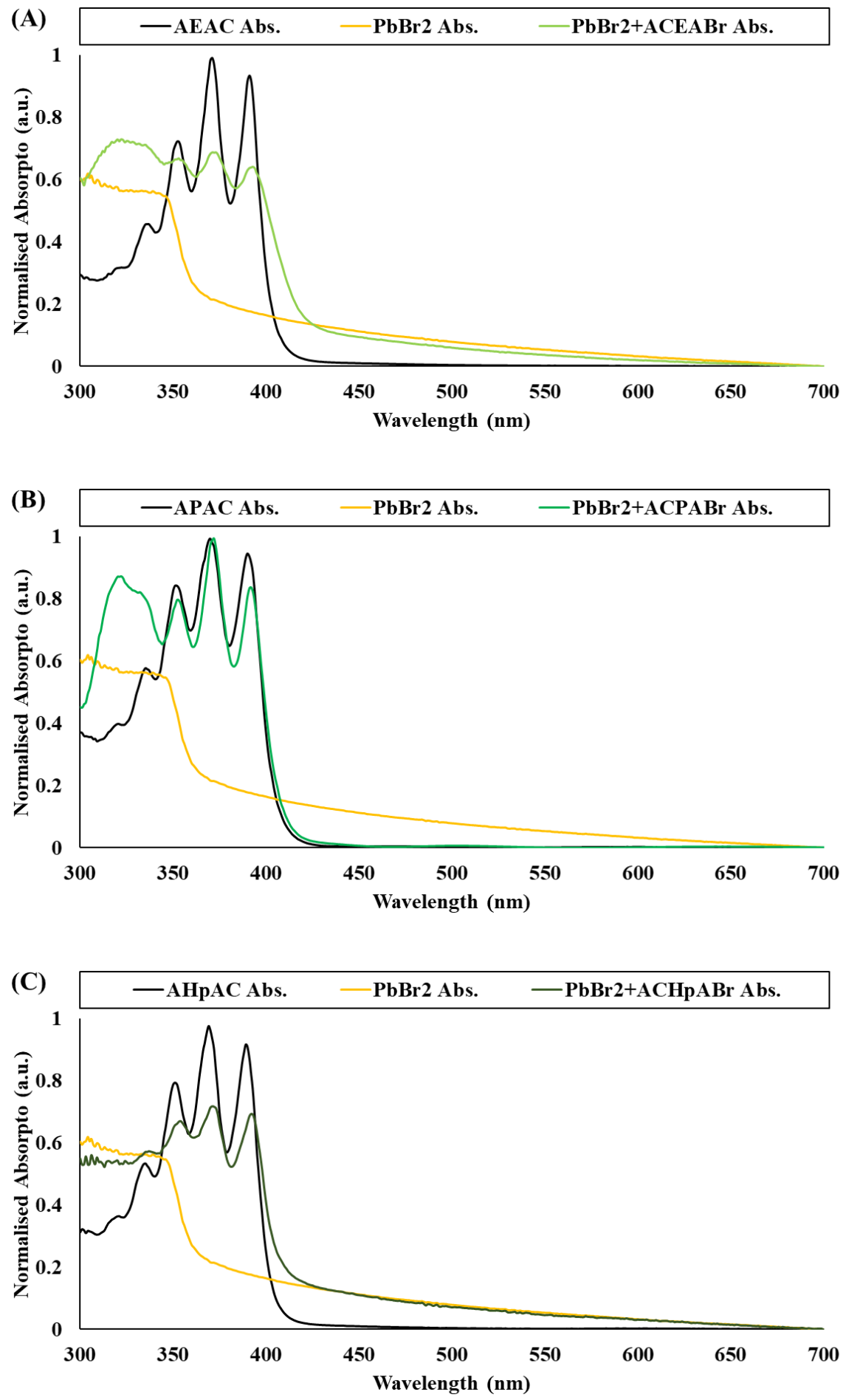

Figure 9d: (A) Comparison between the absorption of pure films of AEAC and $\mathrm{PbBr}_{2}$ with that of a film of $\mathrm{PbBr}_{2}+\mathrm{ACEABr}$. (B) Comparison between the absorption of pure films of APAC and $\mathrm{PbBr}_{2}$ with that of a film of $\mathrm{PbBr}_{2}+\mathrm{ACPABr}$. (B) Comparison between the absorption of pure films of AHpAC and $\mathrm{PbBr}_{2}$ with that of a film of $\mathrm{PbBr}_{2}+\mathrm{ACHpABr}$.

these reasons, and based upon existing literature, that absorption feature was attributed to photodimerization of anthracene. The similarity of these structures suggests that the presence of $\mathrm{PbBr}_{2}$ 
could facilitate the dimerization of the AEAC and APAC molecules in their film form. This could also explain the sharp absorption peak for $\mathrm{PbCl}_{2}+\mathrm{ACEACl}$ and suggest that $\mathrm{PbCl}_{2}$ provides a similar effect, though only in one of the three chloride samples. In this hypothesis, the lack of similar absorption band in the sample $\mathrm{PbBr}_{2}+\mathrm{ACHpABr}$ is due to the presence of the longer heptyl chain, producing a steric effect that forces apart anthracene functional groups and prevents dimerization Beyond $360 \mathrm{~nm}$, the absorption spectrum of all three bromide samples perfectly matches that of the pure AAAC films, once again supporting the conclusion that there is no perovskite phase for the bromide films.

The $\mathrm{PbI}_{2}+$ ACAAI films are unique with regards to the previously discussed chloride and bromide series of films. The characteristic anthracene absorption peaks are less well defined in the iodide series, especially for $\mathrm{PbI}_{2}+\mathrm{ACHpAI}$ in which none of these characteristic peaks are observable for this series of samples, it appears that the presence of lead iodide $\left(\mathrm{PbI}_{2}\right)$ interferes with and distorts the absorption signature of anthracene. This may be due to the similar range of wavelengths that pure $\mathrm{PbI}_{2}$ absorbs when compared to anthracene (see Figure 9e). There are a few discrepancies between the pure $\mathrm{PbI}_{2}$ absorption and the mixed samples. Pure $\mathrm{PbI}_{2}$ displays a broad absorption band between 400 and $450 \mathrm{~nm}$ and a shoulder peak at $500 \mathrm{~nm}$. None of the $\mathrm{PbI}_{2}+\mathrm{ACAAI}$ samples possess these features. Instead the absorption steadily increases from $700 \mathrm{~nm}$ to $\sim 440 \mathrm{~nm}$ and is featureless. It is unlikely that simple scattering of the source light by the sample could account for this lack absorption features as the signal to noise ratio of the sample is low. Alternative explanations include differing morphology and particle sizes of the lead iodide in the mixed phase, resulting in a change in absorption behaviour. 

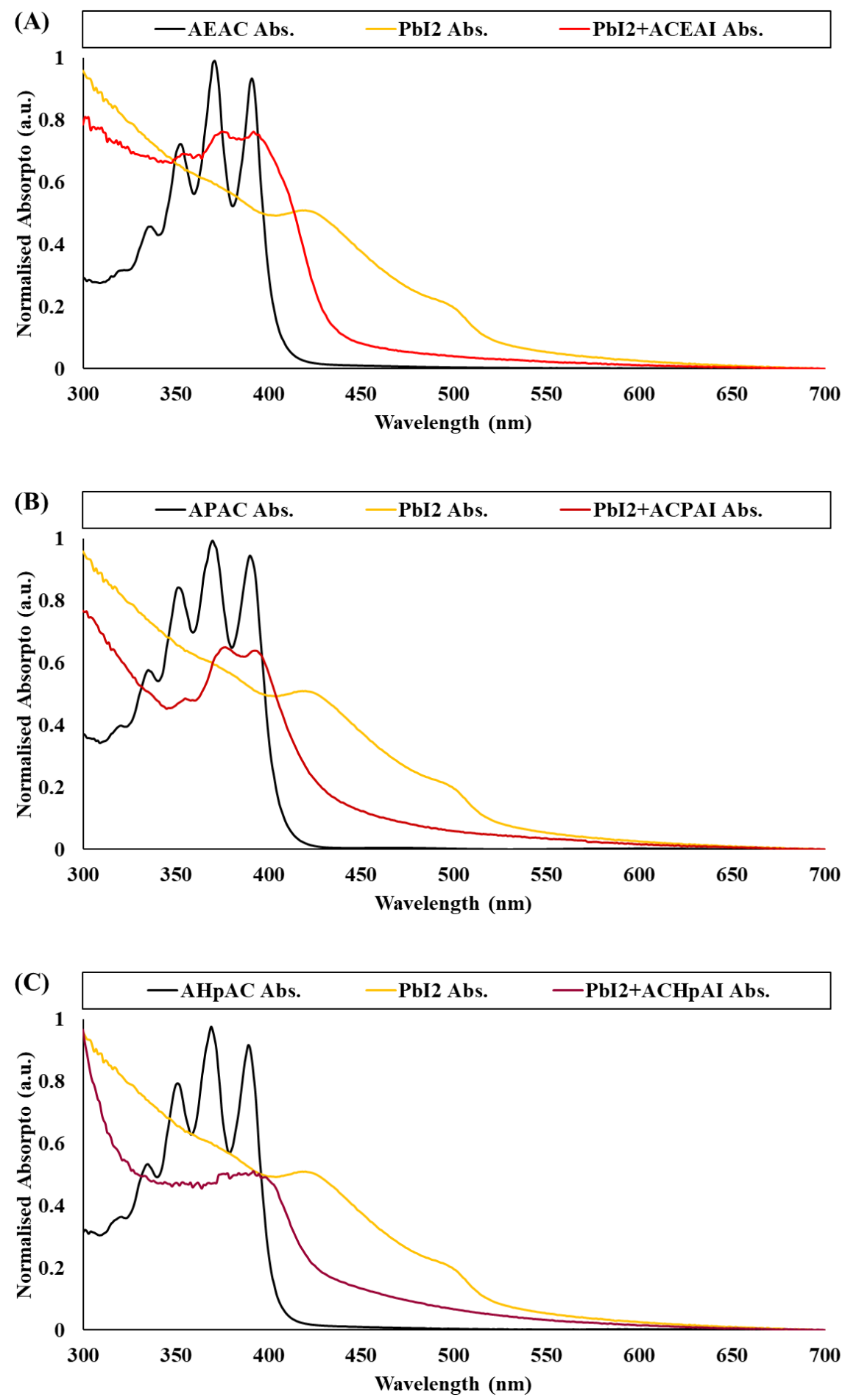

Figure 9e: (A) Comparison between the absorption of pure films of $A E A C$ and $\mathrm{PbI}_{2}$ with that of a film of $\mathrm{PbI}_{2}+$ ACEAI. (B) Comparison between the absorption of pure films of APAC and $\mathrm{PbI}_{2}$ with that of a film of $\mathrm{PbI}_{2}+$ ACPAI. (B) Comparison between the absorption of pure films of AHpAC and $\mathrm{PbI}_{2}$ with that of a film of $\mathrm{PbI}_{2}+\mathrm{ACHpAI}$. 


\section{Photoluminescence of (Anthracene-9-Carboxamido)Alkylaminium Lead Halide Thin-Films}

The emission spectrum of the nine thin-film samples show an interesting series of trends with relation to the length of the alkyl chain in the $N$-(aminoalkyl)anthracene-9-carboxamide (AAAC) molecule and the halogen present. For the chlorides, the $\mathrm{PbCl}_{2}+\mathrm{ACEACl}$ film is unique in that the emission of this sample shows a similar broadened and ill-defined shape as the pure AEAC film. For the other films, the emission mirrors that of solution based AAAC molecules, though slightly red shifted by around $6 \mathrm{~nm}$. This indicates that in the $\mathrm{PbCl}_{2}+\mathrm{ACEACl}$ film, the anthracene functional groups are close enough to allow for the formation of excimers that produce the deeply redshifted and featureless emission spectrum. However, the peak emission wavelength for this sample is $498 \mathrm{~nm}$ vs $534 \mathrm{~nm}$ for the pure AEAC film, and the emission spectrum is less broadened. This is indicative that the incorporation of lead chloride $\left(\mathrm{PbCl}_{2}\right)$ into the film has reduced the orbital overlap between the anthracene functional groups that allow for excimer formation. For the samples $\mathrm{PbCl}_{2}+\mathrm{ACPACl}$ and $\mathrm{PbCl}_{2}+\mathrm{ACHpACl}$ the anthracene functional groups in these films are almost completely unable to form an excimer. As a result, the molecules fluoresce as monomers, producing an emission spectrum reminiscent of the solution based measurements (see Figure $\mathbf{9 g}$ ).

Comparing $\mathrm{PbCl}_{2}+\mathrm{ACHpACl}$ to $\mathrm{PbCl}_{2}+\mathrm{ACPACl}$ shows that compared to the later, the former's emission is less well defined and slightly redshifted. Specifically, the emission for $\mathrm{ACPA}_{2} \mathrm{PbCl}_{4}$ is completely lacking the $\sim 410 \mathrm{~nm}$ emission peak, indicating that the shorter length of the propyl chain is allowing for weak excimer formation. Such a trend is mirrored in the pure AAAC films, in which the formation of excimers becomes less favoured as the alkyl chain increases in length (see Figure 9f). Therefore, it can be concluded that the incorporation of $\mathrm{PbCl}_{2}$ into these films disfavours excimer formation and increases monomer fluorescence.<smiles>NCCNC(=O)c1c2ccccc2cc2ccccc12</smiles><smiles>NCCCNC(=O)c1c2ccccc2cc2ccccc12</smiles><smiles>CC(=O)NCCCCCCCN</smiles>

Figure 9f. Series of $\mathrm{N}$-(aminoalkyl)anthracene-9-carboxamides (AAAC). a. $\mathrm{N}$-(2aminoethyl)anthracene-9-carboxamide (AEAC), b. N-(3-aminopropyl)anthracene-9carboxamide (APAC), c. N-(7-aminoheptyl)anthracene-9-carboxamide (AHpAC). 

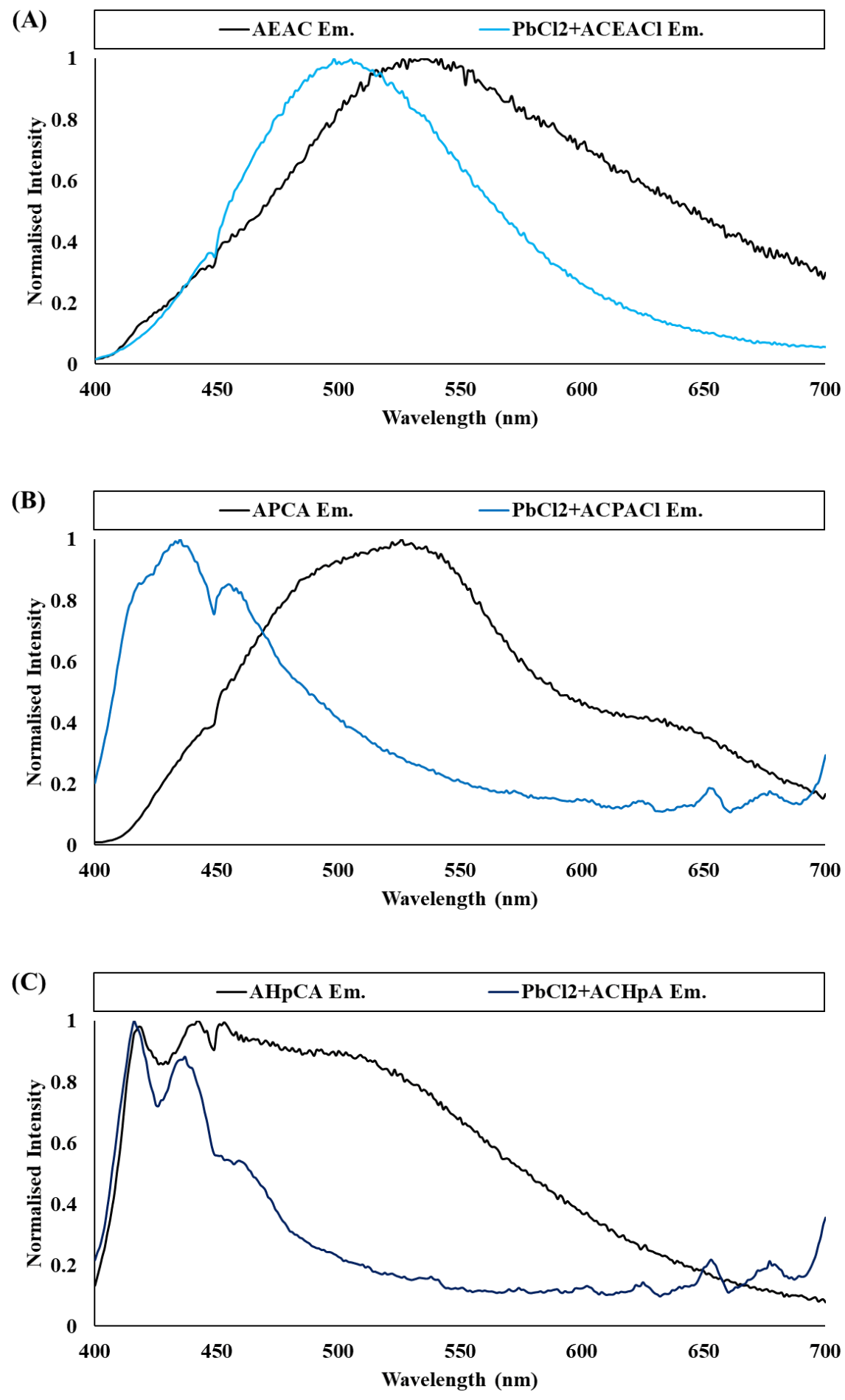

Figure 9g: (A) Comparision between the electronic emission spectra of a pure film of pure $A E A C$ and $\mathrm{PbCl}_{2}+A C E A C l$. (B) Comparision between the electronic emission spectra of a pure film of pure APAC and $\mathrm{PbCl}_{2}+\mathrm{ACPACl}$. (C) Comparision between the electronic emission spectra of a pure film of pure $\mathrm{APAC}$ and $\mathrm{PbCl}_{2}+\mathrm{ACPACl}$. 
The bromide series shows a similar separation between samples that demonstrate excimer emission and monomer emission (see Figure $9 \boldsymbol{h}$ ). However, while the chloride series only demonstrated excimer emission in the $\mathrm{PbCl}_{2}+\mathrm{ACEACl}$ film, both $\mathrm{PbBr}_{2}+\mathrm{ACEABr}$ and $\mathrm{PbBr}_{2}+\mathrm{ACPABr}$ demonstrate redshifted emission. While the presence of $\mathrm{PbCl}_{2}$ is sufficient to disrupt interactions between neighbouring anthracene functional groups in APAC containing films, lead bromide $\left(\mathrm{PbBr}_{2}\right)$ appears to be less effective at doing the same. Comparing the fluorescence of $\mathrm{PbBr}_{2}+\mathrm{ACPABr}$ with that of pure APAC shows that the peak absorbance for the former is blue shifted by $20 \mathrm{~nm}$ relative to the latter. This blue-shift shows that $\mathrm{PbBr}_{2}$ does have a negative influence on excimer formation, but not to the extent of lead chloride. The emission spectrum for $\mathrm{PbBr}_{2}+\mathrm{ACHpABr}$ looks almost identical to that of $\mathrm{PbCl}_{2}+\mathrm{ACHpACl}$, indicating that the combination of the long heptyl chain and the presence of either $\mathrm{PbCl}_{2}$ or $\mathrm{PbBr}_{2}$ is sufficient to completely prevent excimer formation.

For the iodides, the emission from all three samples is dominated by excimer fluorescence (see Figure 9i). The peak fluorescence for all three films matches that of pure AECA, indicating that the negative influence on excimer formation due to the presence of long alkyl chains has been overcome by the presence of $\mathrm{PbI}_{2}$. Therefore, it must be concluded that the presence of lead iodide facilitates excimer formation, as opposed to hindering it in the case of $\mathrm{PbCl}_{2}$ and $\mathrm{PbBr}_{2}$. Such a trend must be related to how the lead halides co-precipitate with the ACAAX salts from the precursor solutions. For the chlorides and to a lesser extent the bromides as well, $\mathrm{PbCl}_{2}$ and $\mathrm{PbBr}_{2}$ must act to isolate the larger AAAC derivatives by crystallising in between the molecules. At the other end of series, the larger iodide anions may be too large to allow for the crystallisation of $\mathrm{PbI}_{2}$ within a molecular matrix, and therefore possibly grow to exclude and concentrate the AAACs together. 

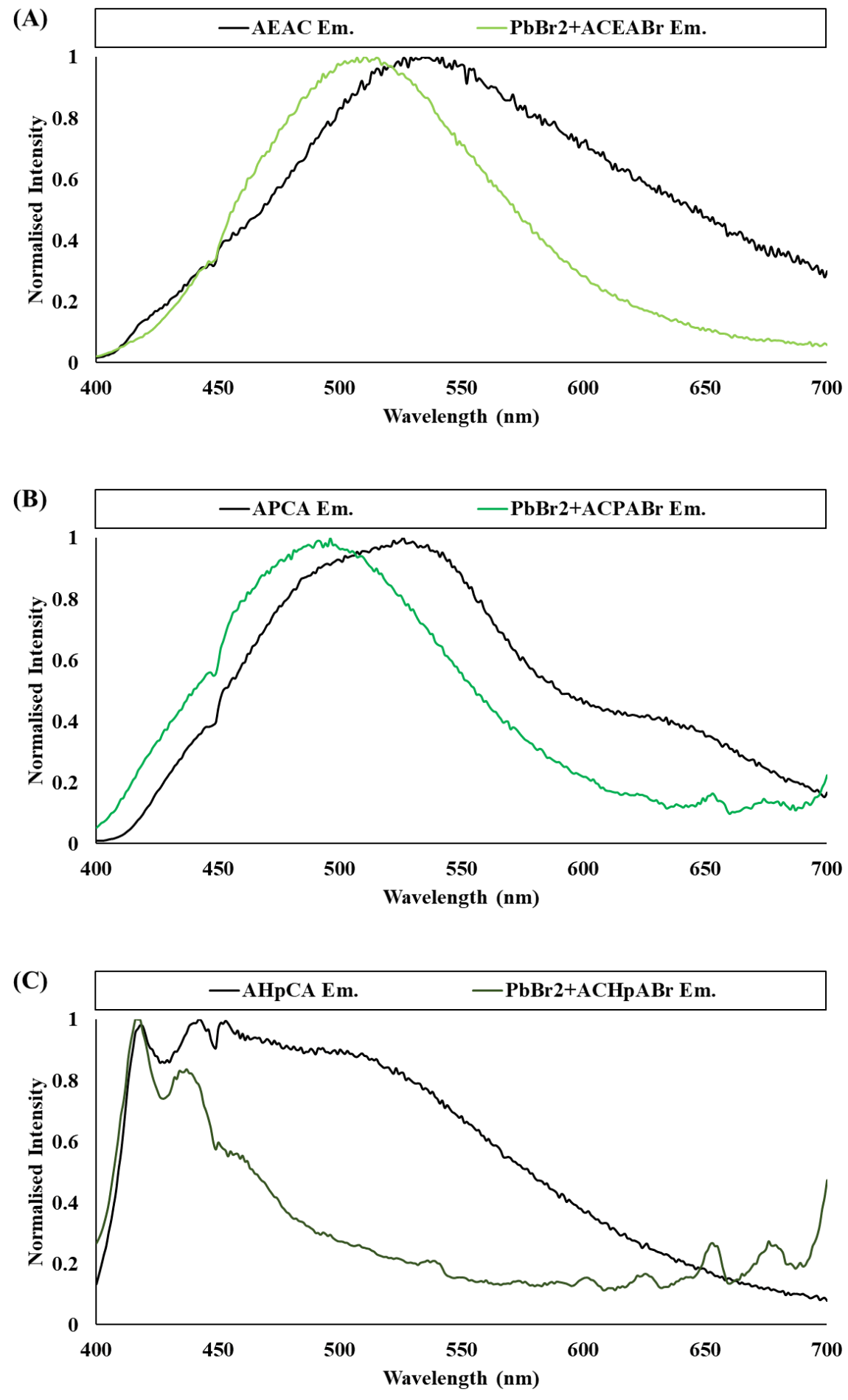

Figure 9h: (A) Comparision between the electronic emission spectra of a pure film of pure $A E A C$ and $\mathrm{PbBr}_{2}+A C E A B r$. (B) Comparision between the electronic emission spectra of a pure film of pure APAC and $\mathrm{PbBr}_{2}+A C P A B r$. (C) Comparision between the electronic emission spectra of a pure film of pure $\mathrm{APAC}$ and $\mathrm{PbBr}_{2}+\mathrm{ACPABr}$. 

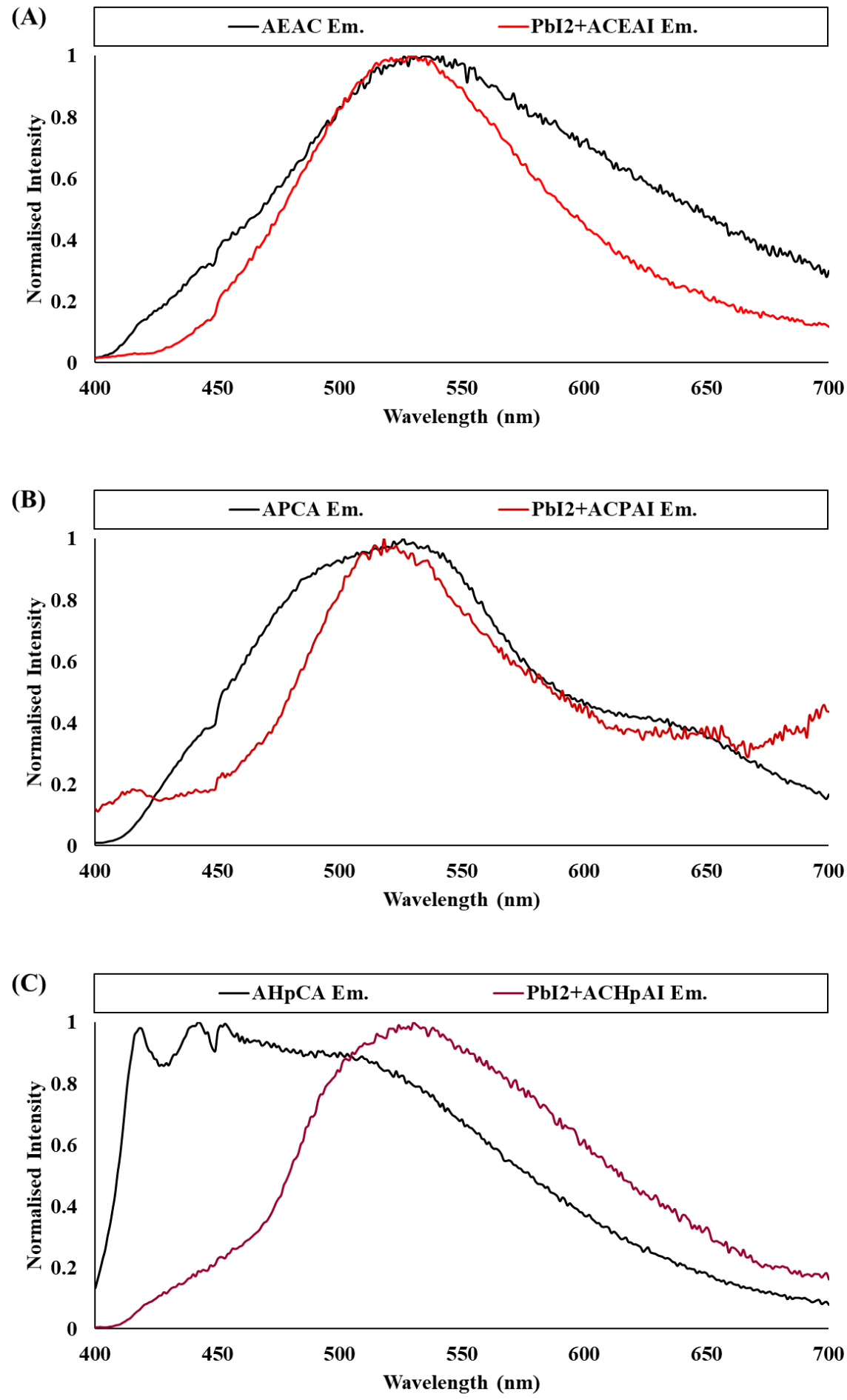

Figure 9i: (A) Comparision between the electronic emission spectra of a pure film of pure AEAC and $\mathrm{PbI}_{2}+$ ACEAI. (B) Comparision between the electronic emission spectra of a pure film of pure APAC and PbI ${ }_{2}+A C P A I$. (C) Comparision between the electronic emission spectra of a pure film of pure APAC and $\mathrm{PbI}_{2}+A C P A I$. 


\section{Electronic Excitation of (Anthracene-9-Carboxamido)Alkylaminium Lead Halide Thin-Films}

The excitation spectra across the complete series of perovskites is highly variable, even when comparing based upon halogen or alkyl chain length. All six chloride and bromide samples produce excitation spectra that share negative correlations with both the corresponding absorption spectrum and the excitation spectrum of the pure AAAC film (see Figures $\mathbf{9 j}, \mathbf{9 k}$, and $9 \boldsymbol{l}$ ). Such a result indicates the presence of dark states excimers, interactions between anthracene functional groups that do not result in fluorescence.

A negative correlation between excitation and absorption holds true for the short wavelength absorption bands displayed by $\mathrm{PbCl}_{2}+\mathrm{ACEACl}, \mathrm{PbBr}_{2}+\mathrm{ACEABr}$, and $\mathrm{PbBr}_{2}+\mathrm{ACPABr}$. In addition, the $420 \mathrm{~nm}$ excitation peak displayed by $\mathrm{PbCl}_{2}+\mathrm{ACEACl}$ is indicative of an excimer. Such results support the hypothesis that these absorption bands are associated with interactions between neighbouring anthracene functional groups. In addition to the lack of excimer emission, these samples also demonstrate unique excitation spectra. While all excimer emitting samples display low excitation intensity between 300 and $~ 325 \mathrm{~nm}$, all three monomer emitting samples show relatively high excitation intensities at this region. Additionally, while these samples display the overall negative correlation in excitation spectral peaks with the pure AAAC films between 350 and $400 \mathrm{~nm}$, at the shorter wavelength region there is a positive correlation. This suggests that the lead halide present within these samples reduces excimer formation rates, but also increases the transparency of the sample to high energy wavelengths, facilitating increased excitation intensity in that spectral region.

A unique excitation feature demonstrated by the bromide samples $\mathrm{PbBr}_{2}+\mathrm{ACEABr}$ and $\mathrm{PbBr}_{2}+\mathrm{ACPABr}$ is that the intensity of the excitation spectrum increases $\sim 310 \mathrm{~nm}$ to $300 \mathrm{~nm}$. Subsequent excitation scans with an extended range into the ultra-violet range showed that this was part of a larger feature that peaks at $295 \mathrm{~nm} . \mathrm{PbBr}_{2}+\mathrm{ACPABr}$ demonstrates a less significant shoulder peak at $\sim 292 \mathrm{~nm}$. Initially, these features were considered to be excitation peaks, however, the negative correlation observed between excitation and absorption in these samples suggest that this 'peak' is actually a broad band that is cut off due anthracene absorption and subsequent selfquenching.

The excitation measurements on the iodide samples display a high signal to noise ratio, a result of the low fluorescence of these samples. Two of the iodide samples, $\mathrm{PbI}_{2}+\mathrm{ACEAI}$ and $\mathrm{PbI}_{2}+\mathrm{ACPAI}$, display a positive correlation between the spectral peaks of their excitation spectra and that of the pure AAAC film. One explanation for this is the significant overlap between the absorption spectra of $\mathrm{PbI}_{2}$ and the AAACs. $\mathrm{PbI}_{2}$ strongly absorbs in a similar region of the spectrum to the anthracene functional group and therefore could be preventing significant excitation of chromophore. Therefore, the wavelengths of light that are more likely to induce anthracene excitation within the film are those that 
are principally absorbed by anthracene. This explanation would also explain the lack of fluorescence by these samples.

However, peak excitation of these samples still correlates with reduced absorption at $\sim 350 \mathrm{~nm}$. This suggests that the self-quenching that results from high concentrations of anthracene is still having an influence on the excitation of the samples.

Unlike the other two iodide samples, $\mathrm{PbI}_{2}+\mathrm{ACHpAI}$ shares a negative correlation between its excitation spectrum and that of pure AHpAC. Why this particular sample breaks this trend is unclear, but it may be related to the large alkyl chains of the AHpAC molecule, as this is the only noncommon factor between this sample and the others. It may be that the long alkyl chain prevents the organic and inorganic components of the film from sufficiently mixing, resulting in high concentrations of ACHpAI that is relatively lead free. This may be sufficient to increase the selfquenching effect of anthracene and produce the negative correlation.

Overall, the excitation measurements of the nine samples does not suggest the presence of a perovskite phase. However, these results do provide useful lessons for the synthesis of anthracene possessing 2D Ruddlesden-Popper perovskite films, namely, that high concentrations of anthracene functional groups can prove to be detrimental to emission, and the full conversion of $\mathrm{PbI}_{2}$ into a perovskite will be necessary to reduce parasitic absorption of light. 

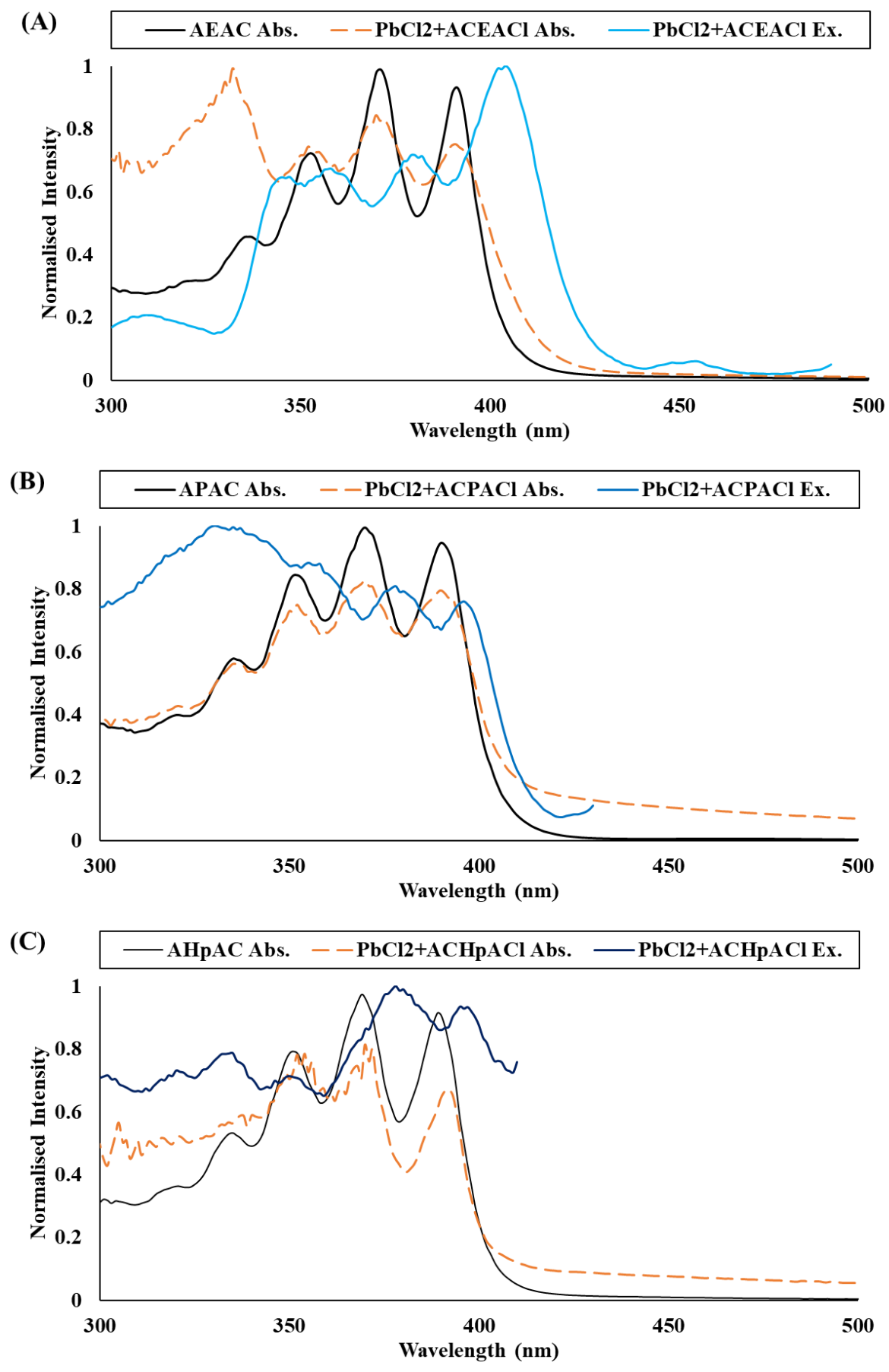

Figure 9j: (A) Comparison between the electronic excitation and absorption spectrum of $\mathrm{PbCl}_{2}+\mathrm{ACEACl}$ with the excitation spectrum of a pure AEAC film. (B) Comparison between the electronic excitation and absorption spectrum of $\mathrm{PbCl}_{2}+\mathrm{ACPACl}$ with the excitation spectrum of a pure APAC film. (C) Comparison between the electronic excitation and absorption spectrum of $\mathrm{PbCl}_{2}+\mathrm{ACHpACl}$ with the excitation spectrum of a pure AHpAC film. 

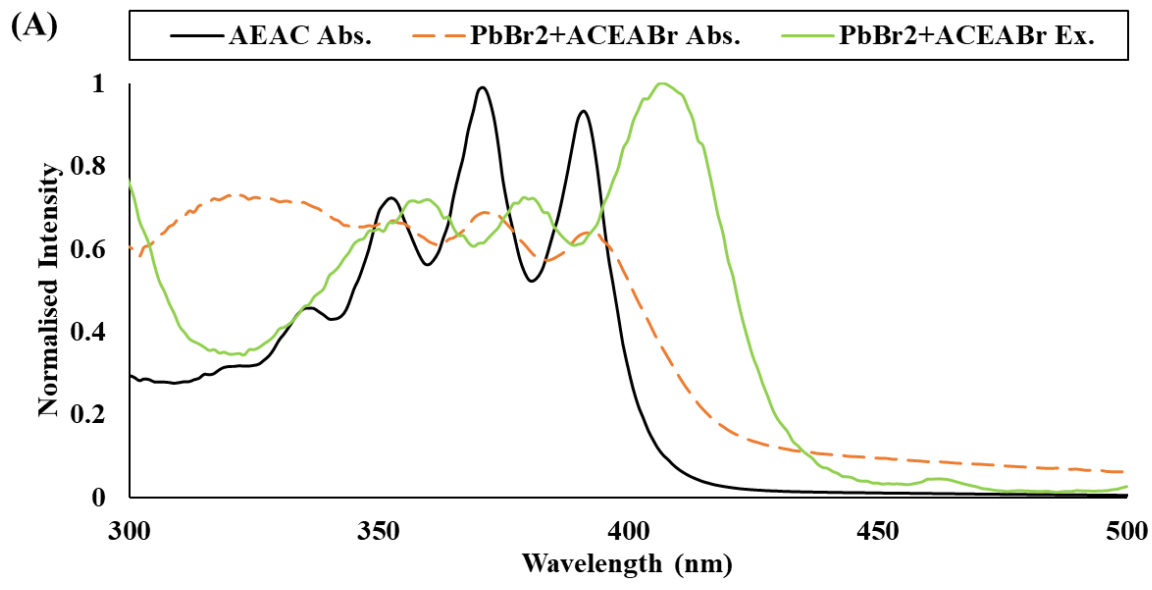

(B) - APAC Abs. - -PbBr2+ACPABr Abs. - PbBr2+ACPABr Ex.

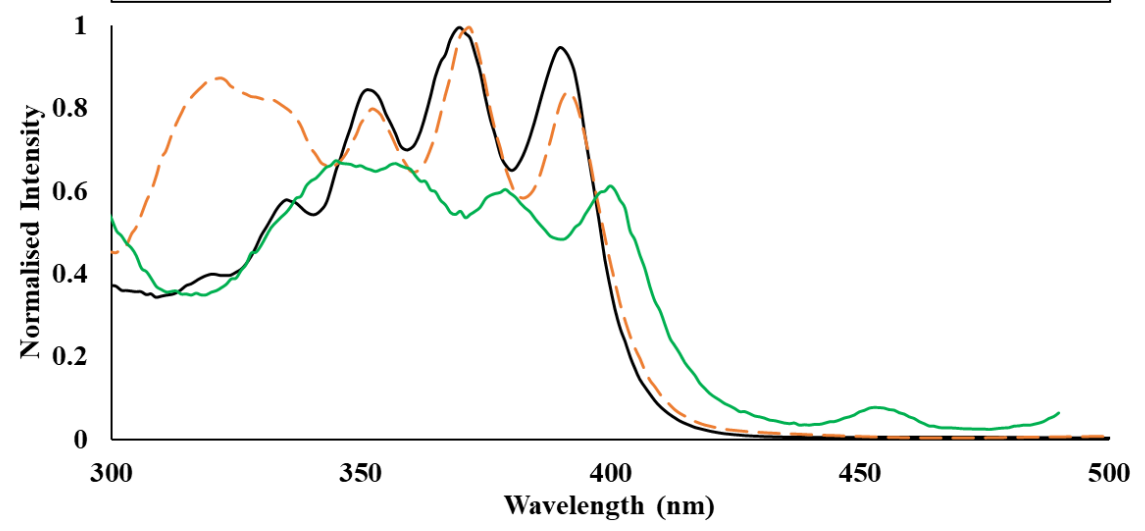

(C) - AHpAC Abs. - - PbBr2+ACHpABr Abs. - PbBr2+ACHpABr Ex.

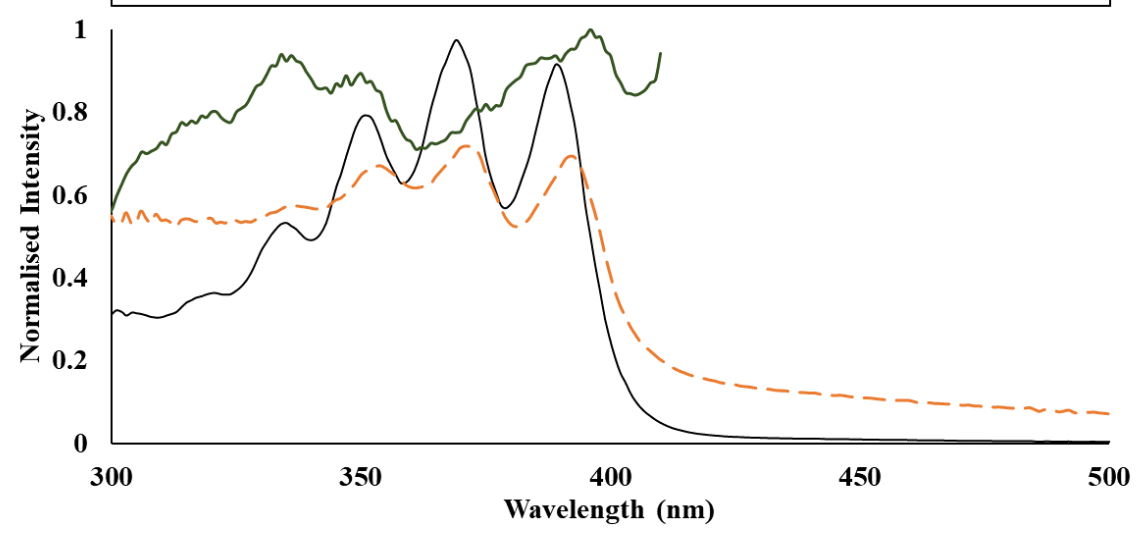

Figure 9k: (A) Comparison between the electronic excitation and absorption spectrum of $\mathrm{PbBr}_{2}+\mathrm{ACEABr}$ with the excitation spectrum of a pure AEAC film. (B) Comparison between the electronic excitation and absorption spectrum of $\mathrm{PbBr}_{2}+\mathrm{ACPABr}$ with the excitation spectrum of a pure APAC film. (C) Comparison between the electronic excitation and absorption spectrum of $\mathrm{PbBr}_{2}+A C H p A B r$ with the excitation spectrum of a pure AHpAC film. 

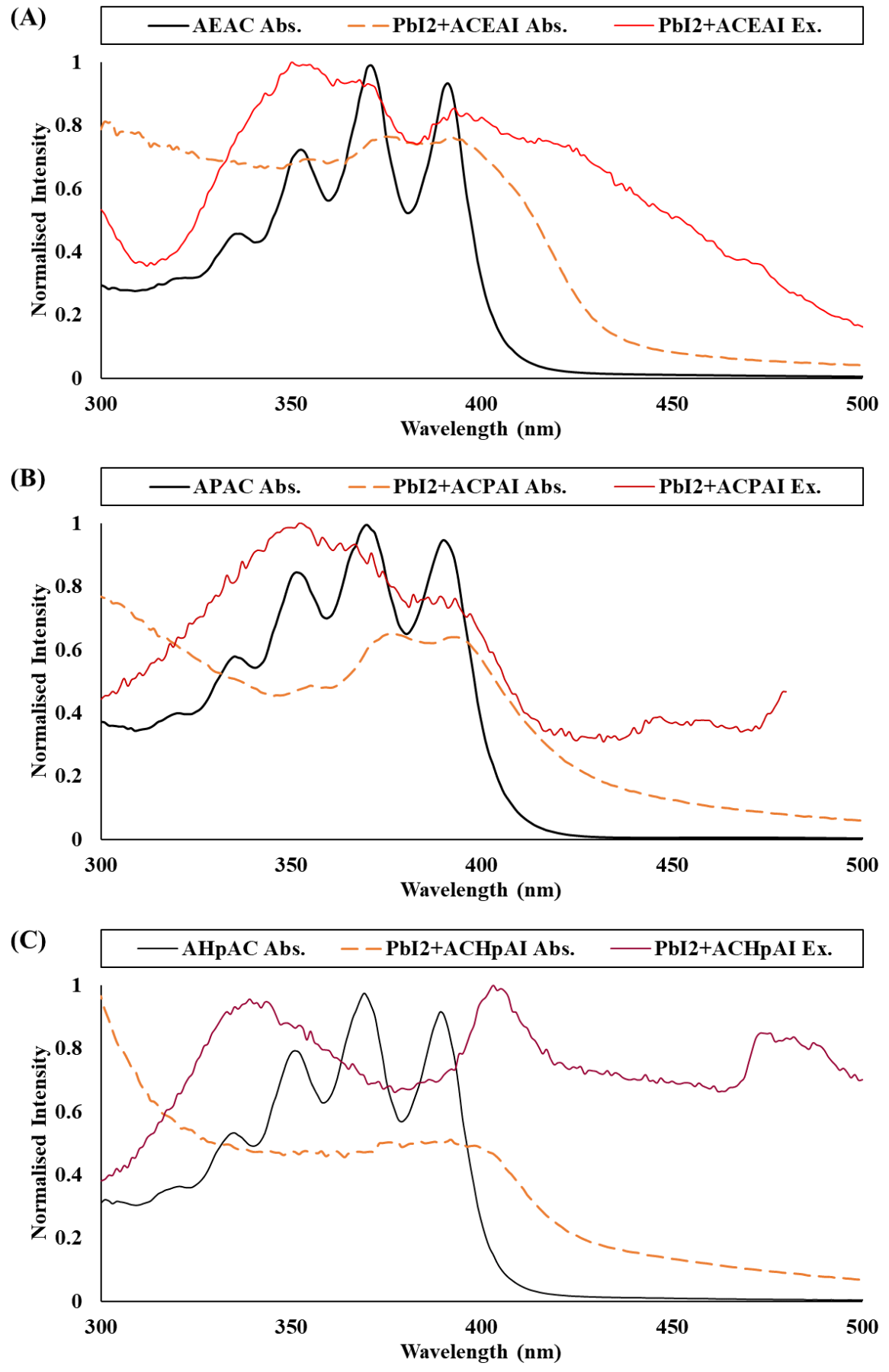

Figure 9l: (A) Comparison between the electronic excitation and absorption spectrum of $\mathrm{PbI}_{2}+A C E A I$ with the excitation spectrum of a pure AEAC film. (B) Comparison between the electronic excitation and absorption spectrum of $\mathrm{PbI}_{2}+\mathrm{ACPAI}$ with the excitation spectrum of a pure APAC film. (C) Comparison between the electronic excitation and absorption spectrum of $\mathrm{PbI}_{2}+\mathrm{ACH} \mathrm{PAI}$ with the excitation spectrum of a pure AHpAC film. 


\section{Photoluminescence Lifetimes of (Anthracene-9-Carboxamido)Alkylaminium Lead Halide Thin-} Films

As can be seen in Figure 9m, the emission decay of the nine samples display several trends. Most notable is the significant difference in emission decay between samples that demonstrates excimer emission versus monomer emission. All three samples possessing the AEAC species demonstrates excimer emission with a comparably slow rate of decay. Both the chloride and bromide samples demonstrate comparable decay rates while the iodides rate is substantially lower.

The series of samples possessing APCA molecules demonstrates greater emission variability, with the chloride sample $\mathrm{PbCl}_{2}+\mathrm{ACPACl}$ exhibiting monomer emission and the other two samples demonstrating excimer emission. There is a clear difference in emission decay between the chloride sample and both the bromide and iodide samples. The chloride sample possess a significantly faster rate of emission decay compared to the other two samples and the pure APAC film. The rate of decay for an excimer species is highly dependent on the relative orientation of the individual anthracene functional groups that form the excimer. Long lifetime excimer emission tends to be the result of a 'sandwich' orientation, in which the pair of anthracene functional groups are parallel to each other . The short lifetime of $\mathrm{PbCl}_{2}+\mathrm{ACPACl}$ when compared to the pure APAC film lends further credence to the hypothesis that the incorporation of lead chloride disrupts the crystallisation of the anthracene molecules and prevents excimer formation.

Referring back to the possible orientation of the AAAC molecules, if indeed the anthracene functional groups are orientated in a 'sandwich', that would facilitate photodimerization, which has been shown to correlate well with the short wavelength absorption bands demonstrated by the pure solution of $\mathrm{AEAC}$, and the thin-film samples $\mathrm{PbCl}_{2}+\mathrm{ACEACl}, \mathrm{PbBr}_{2}+\mathrm{ACEABr}$, and $\mathrm{PbBr}_{2}+\mathrm{ACPABr}$.

The samples containing AHpAC molecules demonstrates the same pattern as seen in the APAC series. All samples that demonstrate monomer emission display short fluorescence lifetimes while those that display excimer emission produce long decay times. Once again, this result supports the role that lead chloride $\left(\mathrm{PbCl}_{2}\right)$ and lead bromide $\left(\mathrm{PbBr}_{2}\right)$ have in preventing excimer formation between neighbouring APAC and AHpAC molecules.

In both the chloride and bromide samples, there is a clear negative correlation between increasing alkyl chain length and emission decay. For the iodides, all three samples demonstrate similar rates of emission decay, with the exception being $\mathrm{PbI}_{2}+\mathrm{ACPAI}$ which narrowly exceeds the others. However, the background noise of these samples is significant, a consequence of the dimness of their fluorescence. 

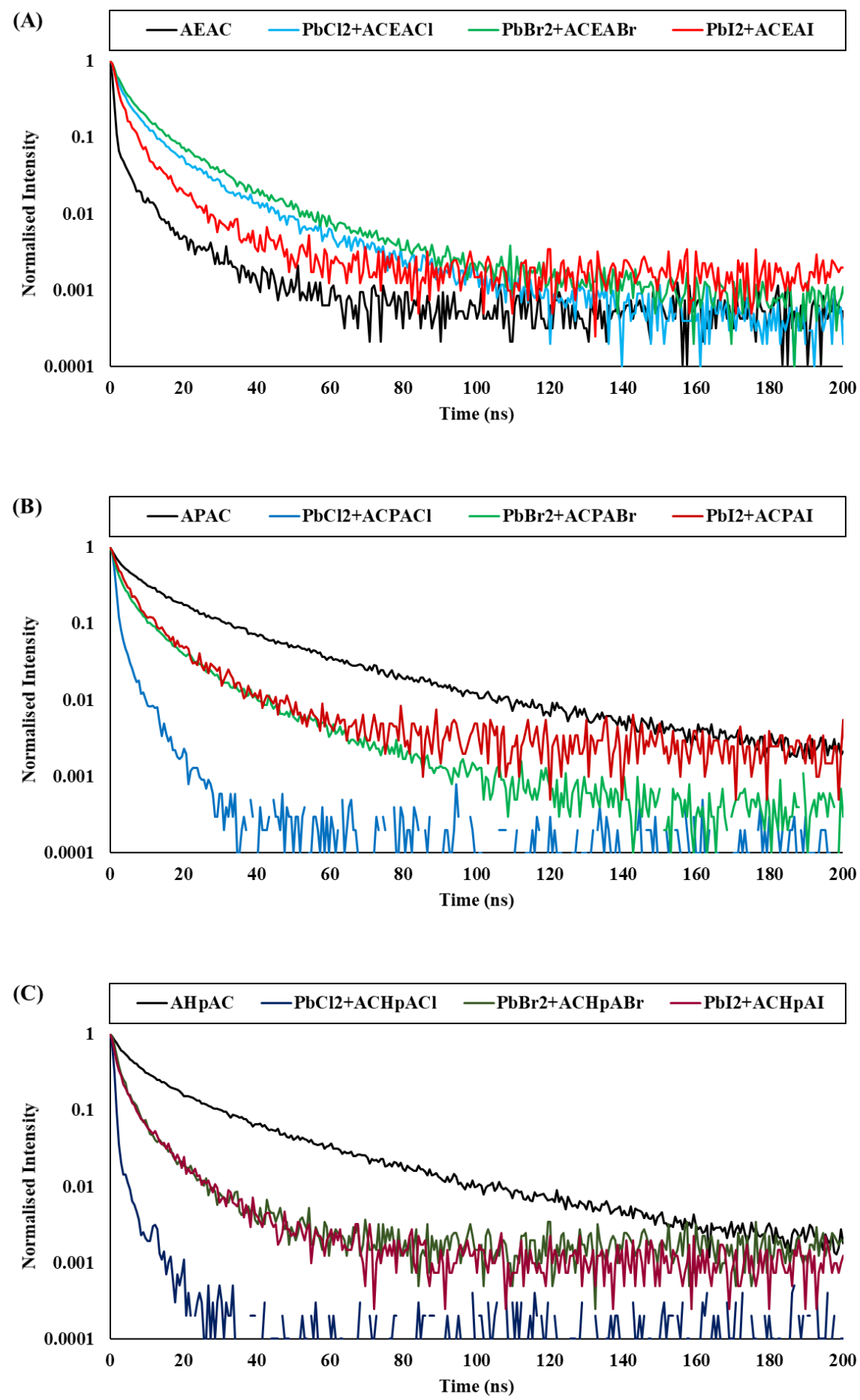

Figure 9m. (A) Comparison between the decay rates of all three PbX $X_{2}+A C E A X$ samples with that of a pure AEAC film. (B) Comparison between the decay rates of all three $P b X_{2}+A C P A X$ samples with that of a pure APAC film. (C) Comparison between the decay rates of all three $P b X_{2}+A C H p A X$ samples with that of a pure AHpAC film. 


\section{Photoluminescence Quantum Yield of (Anthracene-9-Carboxamido)Alkylaminium Lead Halide} Thin-Films

Photoluminescent quantum yield (PLQE) was measured for all samples. The wavelengths of light used to excite the samples were based upon the peak excitation wavelengths of those samples. For the iodides, two measurements were performed, one using $400 \mathrm{~nm}$ light and the other utilising $300 \mathrm{~nm}$ light.

All iodide samples produced PLQEs of $0 \%$ for both wavelengths, showing that these samples suffer from many non-radiative loss mechanisms. Such a result would be a consequence of the fluorescence quenching due to the high concentration of AAAC in the sample, the strong absorbance by lead iodide $\left(\mathrm{PbI}_{2}\right)$, and conditions that are conducive to dark state excimer formation, and the possible presence of trap-states.

All samples containing AHpAC produced PLQEs of $0 \%$.

Illuminating the chloride samples $\mathrm{PbCl}_{2}+\mathrm{ACEACl}$ and $\mathrm{PbCl}_{2}+\mathrm{ACPACl}$ with $400 \mathrm{~nm}$ light produced photoluminescent quantum yields of 0.94 and $1.07 \%$. Illuminating $\mathrm{PbCl}_{2}+\mathrm{ACPACl}$ with $330 \mathrm{~nm}$ light to target the $~ 330 \mathrm{~nm}$ excitation band produced a PLQE of $4.67 \%$.

Exciting the bromide samples $\mathrm{PbBr}_{2}+\mathrm{ACEABr}$ and $\mathrm{PbBr}_{2}+\mathrm{ACPABr}$ with $400 \mathrm{~nm}$ light produces similar levels of performance as the chlorides, with PLQEs of $1.3 \%$ and $0.68 \%$ respectively. However, illuminating the samples to target the high energy excitation band at $300 \mathrm{~nm}$ produces PLQEs of $29.39 \%$ for $\mathrm{PbBr}_{2}+\mathrm{ACEABr}$ and $13.46 \%$ for $\mathrm{PbBr}_{2}+\mathrm{ACPABr}$. The dramatic difference in PLQE between exciting the samples at $300 \mathrm{~nm}$ and $400 \mathrm{~nm}$ is likely to be a consequence of the respective degrees of excimer formation and resulting emission versus monomer emission. Exciting the samples at $400 \mathrm{~nm}$ results in low efficiency due to the significant absorbance by anthracene and the resulting quenching. Exciting the sample with $300 \mathrm{~nm}$ allows the light to propagate further through the film and induce more excitation and emission events.

\section{9c. Concluding Remarks on (Anthracene-9-Carboxamido)Alkylaminium Lead Halide ([ACAA $\left.]_{2} \mathbf{P b X}_{4}\right)$ Thin-Films}

From the absorption and photoluminescence data, it is clear that these materials are not perovskites as the material does not demonstrate any of the characteristic photoluminescent properties of $2 \mathrm{D}$ Ruddlesden-Popper perovskites; such as a strong absorption peak and corresponding excitation features at $300 \mathrm{~nm}$ for chlorides, $400 \mathrm{~nm}$ for bromides, and $550 \mathrm{~nm}$ for iodides. ${ }^{65,66,67}$

Though these are not perovskites, lessons can be learned from these samples for future research. For example, synthesising a perovskite with a high concentration of anthracene runs the risk of selfquenching and reducing the efficiency of the material. However, one benefit to these high 
concentrations is the formation of excimer states. Interactions between excited and ground state anthracene molecules is required for singlet fission to occur. From these results, it can be seen that the shorter chain length of $N$-(2-aminoethyl)anthracene-9-carboxamide supports excimer formation, and increasing the alkyl chain length disfavours it. Therefore, by altering the alkyl chain length, rates of excimer formation can be tuned.

Additionally, the method that was utilised to synthesis the two dimensional perovskites did not work and therefore alternative methods need to be tested. For example, Oswald et al published results on what they refer to as the general synthesis principles for Ruddlesden-Popper perovskites. ${ }^{68}$ In their work, they utilised a $57 \mathrm{wt} \%$ hydroiodic acid (HI) solution to convert lead oxide ( $\mathrm{PbO})$ and several different primary amines into the perovskite phase. Using their method, the authors were able to produce a series of both pure two-dimensional and 2D/3D hybrids. One of the amines they worked with was benzylamine, an aromatic compound. The demonstrated versatility of this method suggests that it may be capable of producing a perovskite phase that includes $N$-(aminoalkyl)anthracene-9carboxamides within the crystal lattice. 


\section{Intercalation of (Anthracene-9-Carboxamido)Alkylaminium Halides}

\section{(ACAAX) into Lead Halide Nanocrystals}

\section{0a. Synthesis of Lead (II) Halide Nanocrystals}

The aim of this method was to produce 2D Ruddlesden-Popper perovskites as colloidal suspensions of nanocrystals. Due to the insolubility of the anthracene derivative in many solvents, including 1octadecene (ODE) and oleic acid (OA), a straightforward hot injection method could not be employed. However, it has been demonstrated that organic halide salts can intercalate into lead (II) halide $\left(\mathrm{PbX}_{2}\right)$ nanocrystals to produce $2 \mathrm{D}$-Ruddlesden halide perovskites. ${ }^{69}$ Therefore, attempts were made to mimic this process by intercalating the anthracene derivatives into lead halide nanocrystals (see Figure 10a).

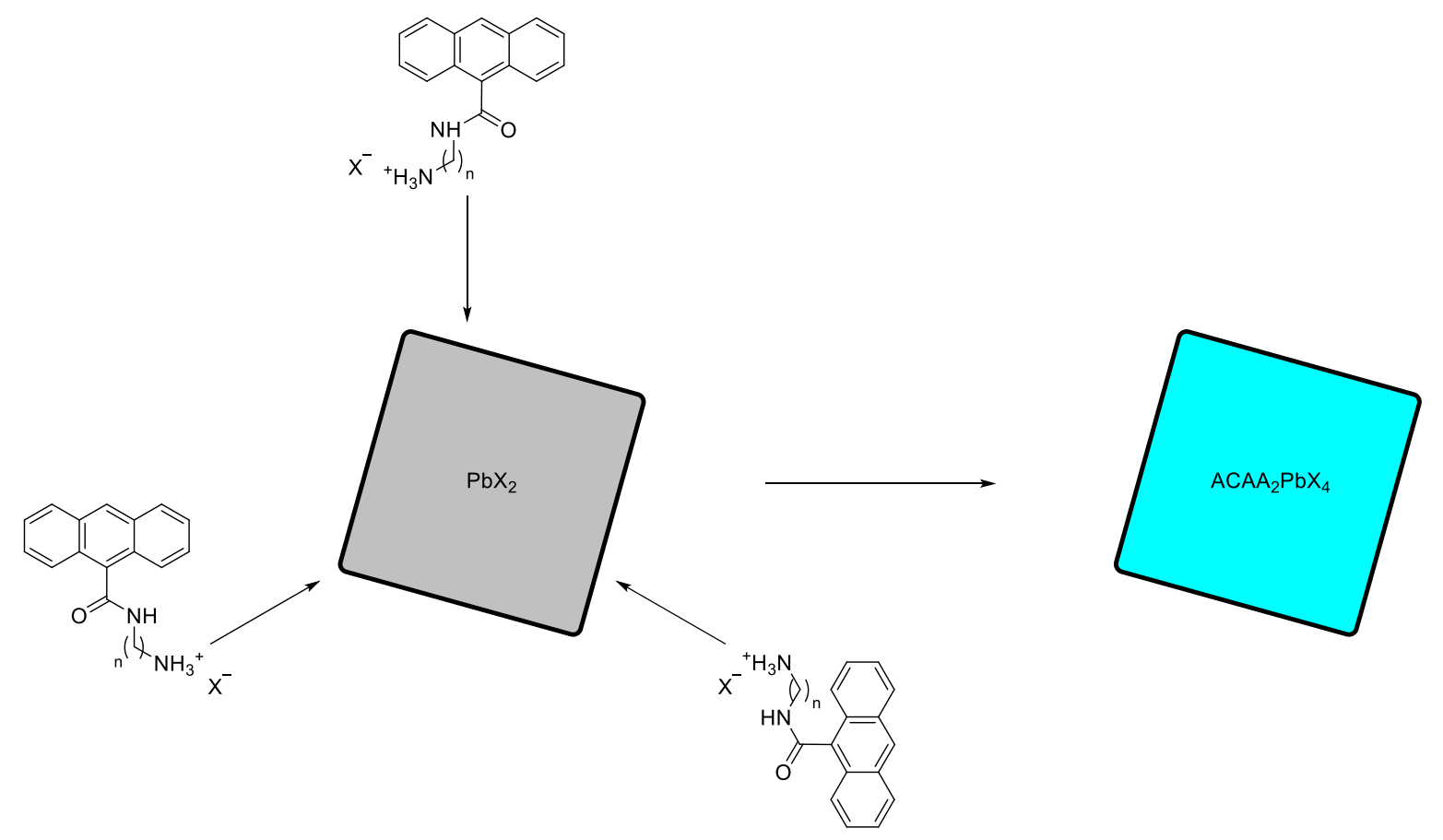

Figure 10a. A graphical scheme depicting the essence of this particular strategy for coupling anthracene functionalised molecules into a perovskite phase. 


\section{Step 1: Lead (II) Oleate Preparation}

To produce the precursor nanocrystals, a solution of lead (II) oleate was produced by reacting $379 \mathrm{mg}$ $(1 \mathrm{mmol})$ of lead (II) acetate $\left(\mathrm{PbOAc}_{2}\right)$ with $3 \mathrm{~mL}$ of OA dissolved within $45 \mathrm{~mL}$ of octadecene (ODE) in a $50 \mathrm{~mL}$ three neck round bottom flask. The reaction vessel was connected to a vacuum line and placed under vacuum and heated to $100^{\circ} \mathrm{C}$ for 1 hour and 30 minutes. Afterwards the reaction was cooled to $80^{\circ} \mathrm{C}$ and placed under a dry nitrogen $\left(\mathrm{N}_{2}\right)$ atmosphere for 1 hour (see Figure $10 \mathrm{~b}$ ).

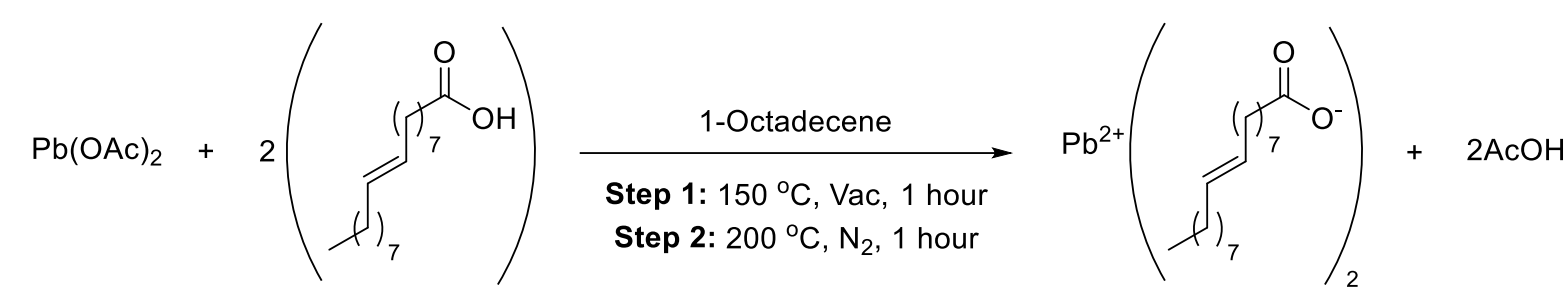

\begin{tabular}{|c|c|c|c|c|c|}
\hline Compound & $\begin{array}{c}\text { Molar } \\
\text { Equivalents }\end{array}$ & mmol & Mass/Volume & $\begin{array}{l}\text { Density } \\
\left(\mathrm{g} \mathrm{mL}^{-1}\right)\end{array}$ & $\begin{array}{c}\text { Molar } \\
\text { Mass } \\
\left(\mathrm{g} \mathrm{mol}^{-1}\right)\end{array}$ \\
\hline $\mathrm{Pb}(\mathrm{OAc})_{2} \cdot 3 \mathrm{H}_{2} \mathrm{O}$ & 1 & 1 & $379.33 \mathrm{mg}$ & - & 379.33 \\
\hline Oleic Acid & 9.5 & 9.5 & $3 \mathrm{~mL}$ & 0.895 & 282.47 \\
\hline 1-Octadecene & 140.6 & 140.6 & $45 \mathrm{~mL}$ & 0.789 & 252.49 \\
\hline
\end{tabular}




\section{Step 2: Preparation of the Halogen Source}

In another vessel, 2 mmol of tetrabutylammonium bromide (TBABr) or tetrabutylammonium iodide (TBAI) along with $3 \mathrm{~mL}$ of ODE and $7 \mathrm{~mL}$ of OAm was placed into a $25 \mathrm{~mL}$ three neck round bottom flask. When synthesising lead chloride nanocrystals, $3 \mathrm{mmol}$ of tetrabutylammonium chloride (TBACl), $4.5 \mathrm{~mL}$ of $\mathrm{ODE}$, and $10.5 \mathrm{~mL}$ of $\mathrm{OAm}$ are required. The reaction vessel was connected to a vacuum line and the mixture was placed under vacuum and heated to $150^{\circ} \mathrm{C}$ for 1 hour and 30 minutes to degas it. Afterwards the mixture was placed under a dry $\mathrm{N}_{2}$ atmosphere and the temperature raised to $200^{\circ} \mathrm{C}$. These conditions were maintained for 1 hour to allow for the halide source to react with the OAm. The reaction was then cooled to no less than $50^{\circ} \mathrm{C}$ to prevent solidification of the product (see Figure 10c).

Molar
Equivalents




\section{Step 3: Lead Halide Nanocrystal Synthesis}

The lead oleate solution was heated to $160-200^{\circ} \mathrm{C}$ (to adjust the synthesised nanocrystal size). The entirety of the halogen source was then injected into the lead oleate solution with vigorous stirring after which the temperature was readjusted to $120-200^{\circ} \mathrm{C}$ (see Figure 10d) and allowed to stir for 30 minutes.

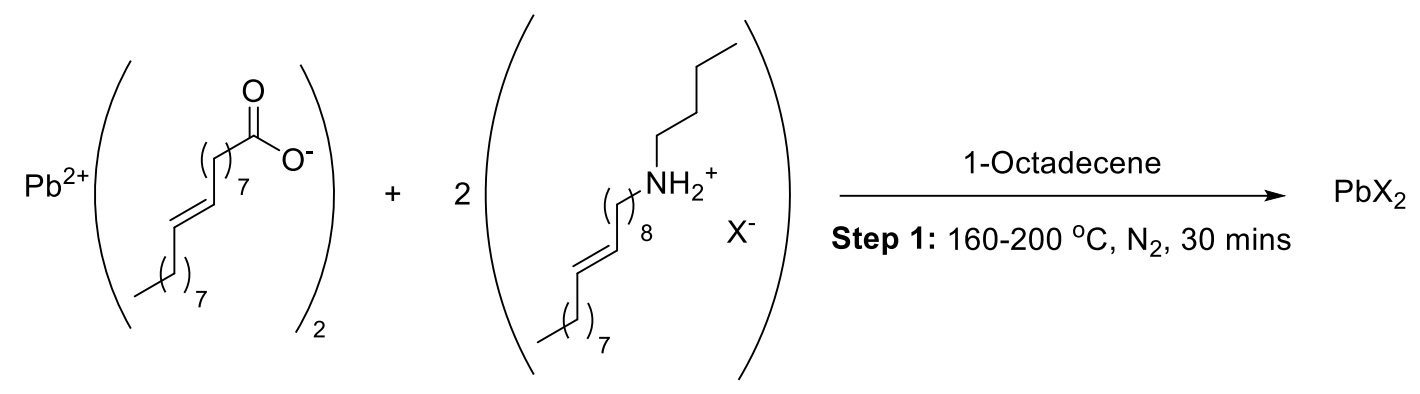

Figure 10d. Formation of lead (II) halide nanoparticles. Note that for the formation of $\mathrm{PbCl}_{2}$ NCs three molar equivalents of the halogen source is required.

\section{Step 4: Washing Procedure}

The reaction mixture was placed within a centrifuge tube and spun at $10000 \mathrm{rpm}$ for 10 minutes. The decant was discarded and the solid was collected and resuspended in toluene. The resulting suspension was then centrifuged at $4000 \mathrm{rpm}$ for 2 minutes. The solid was composed of large noncolloidal particles and so discarded while the decant containing the still suspended nanocrystals was collected. Precipitation of the nanoparticles was then induced by the addition of $n$-butanol $\left({ }^{n} \mathrm{BuOH}\right)$ and centrifuged at $10000 \mathrm{rpm}$ for 5 minutes. The decant was discarded and the solid resuspended in toluene. This washing procedure was repeated at least two more times. A sample of the final nanoparticle solution was deposited onto a pre-weighed glass slide and allowed to dry. This slide was then re-weighed to determine the mass of the resulting precipitate. The mass of the solid divided by the volume of solution deposited provides an approximate concentration of the nanoparticles.

\section{$\underline{\text { Analysis }}$}

All samples had their absorption spectrum measured on a Varian Cary 50 Scan UV/Vis Spectrophotometer, their photoluminescence properties including emission, excitation, photoluminescence quantum yield, and emission lifetime measured on an Edinburgh Instruments FLS980 photoluminescence spectrometer, and their particle morphology analysed by either a Joel 2010 transmission electron microscope or a Joel 2100 transmission electron microscope. Note: While $\mathrm{X}$-Ray Diffraction data was desired, the necessary equipment was unavailable during the duration of this project. 


\section{0b. Results and Discussion of Lead (II) Halide Nanocrystal Synthesis}

\section{Observations}

During the course of the degassing process, the lead acetate was consumed by the reaction with oleic acid (OA), producing a clear and colourless solution that was free of any remaining solid reagent. The reaction mixture mildly bubbled and developed condensation on the walls of the flask. This condensation was most likely acetic acid as this is an expected by-product of the reaction and would easily boil at the reduced pressure and high temperature.

Heating and degassing the oleylamine (OAm) and 1-octadecene (ODE) reaction mixture containing either tetrabutylammonium bromide (TBABr) or tetrabutylammonium iodide (TBAI) does not result in the consumption of either reagent. Only once the reaction mixture is placed under an inert atmosphere of dry nitrogen and heated to at least $190{ }^{\circ} \mathrm{C}$ does the reaction commence. Once at temperature, the solid TBAX is rapidly consumed, resulting in a clear and pale yellow solution with no solid present. $\mathrm{TBACl}$ reacts readily during the degassing process and forms a clear solution with a slight green tinge.

The injection of the iodide source into the lead oleate solution produces an immediate colour change from colourless to golden yellow. Leaving the reaction to stir results in the slow development of a yellow precipitate. Addition of $n$-butanol $(n-\mathrm{BuOH})$ to the reaction mixture and centrifugation isolates a bright yellow solid from a golden yellow supernatant. Suspending the solid in toluene and centrifugation without antisolvent condenses the solid and leaves a clear, yellow, precipitate free solution.

The injection of the bromide source into the lead oleate solution does not produce an immediate colour change. However, the reaction mixture will slowly produce a white precipitate. Resuspension of this solid in toluene and removal of the large particles via centrifugation produces a clear and colourless solution. Evaporating a drop of this solution produces a white film, suggesting that some solid species has been extracted.

The injection of only two molar equivalents of the chloride source does not induce the formation of lead (II) chloride nanocrystals. Three molar equivalents result in the development of a clear solution with a dark grey colour. This solution slowly produces a grey precipitate. The final toluene solution is clear and dark grey. 


\section{Absorption Measurements of Lead Halide Nanocrystals}

All three lead (II) halide nanocrystal solutions display an absorption spectrum that shows increasing absorption with decreasing wavelength. The lead (II) chloride nanocrystal solution $\left(\mathrm{PbCl}_{2} \mathrm{NCs}\right)$ displays a gradual increase in absorption with decreasing wavelength up until $\sim 425 \mathrm{~nm}$ after which the rate of change increases more significantly. Overall, the degree of absorption by $\mathrm{PbCl}_{2} \mathrm{NCs}$ across the visible spectrum accounts for the grey colour of the sample. The solution of lead (II) bromide nanocrystals $\left(\mathrm{PbBr}_{2} \mathrm{NCs}\right)$ is a sample that does not significantly absorb. What absorption spectrum that could be gained shows an almost constant degree of low absorption between 700 and $400 \mathrm{~nm}$, accounting for the complete lack of colour demonstrated by the solution. Beyond $400 \mathrm{~nm}$, the absorption intensity of the sample increases dramatically. Lead (II) iodide nanocrystals do not absorb visible light except at wavelengths shorter than $450 \mathrm{~nm}$. The significant absorption of violet and blue light accounts for the yellow appearance of the sample (see Figure 10e)

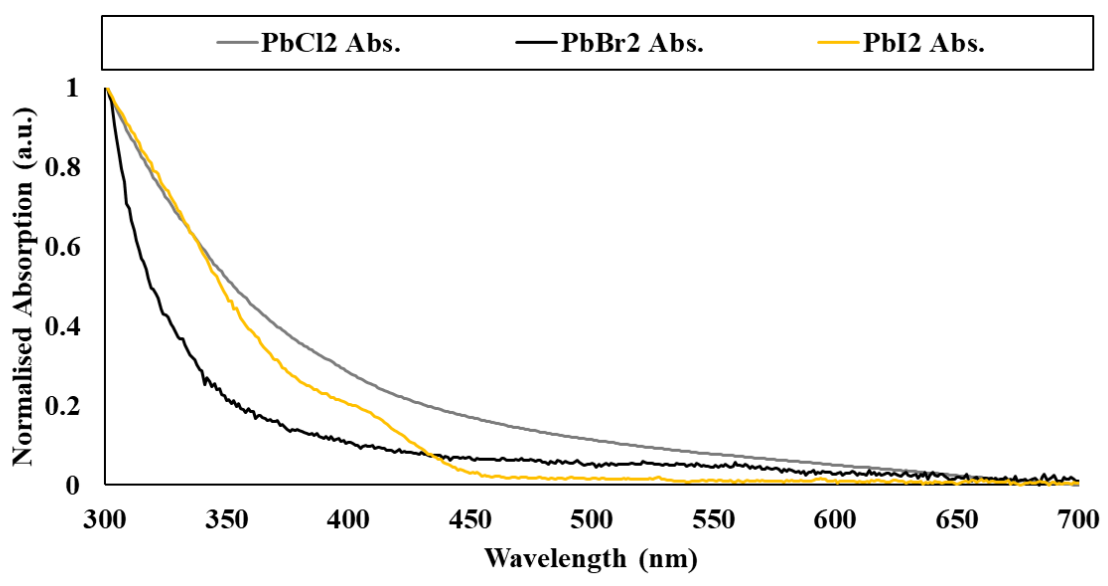

Figure 10e. Overlay of the normalised absorption spectrum of lead chloride, lead bromide, and lead iodide nanocrystals.

Overall, the absorption measurements suggest the successful synthesis of all three lead (II) halide nanocrystals. 


\section{Transition Electron Microscope Images of Lead Halide Nanocrystals}

Images of the solution of $\mathrm{PbCl}_{2}$ nanocrystals shows the solution is composed of cubic particles on the order of 20 to $50 \mathrm{~nm}$ in size. These cubic particles are crystalline in nature as they display detailed lattice fringing (see Figure 10f). Energy-dispersive X-ray spectroscopy (EDS) shows the significant presence of both lead and chlorine, supporting the identification of the product as a solution of lead (II) chloride nanocrystals.

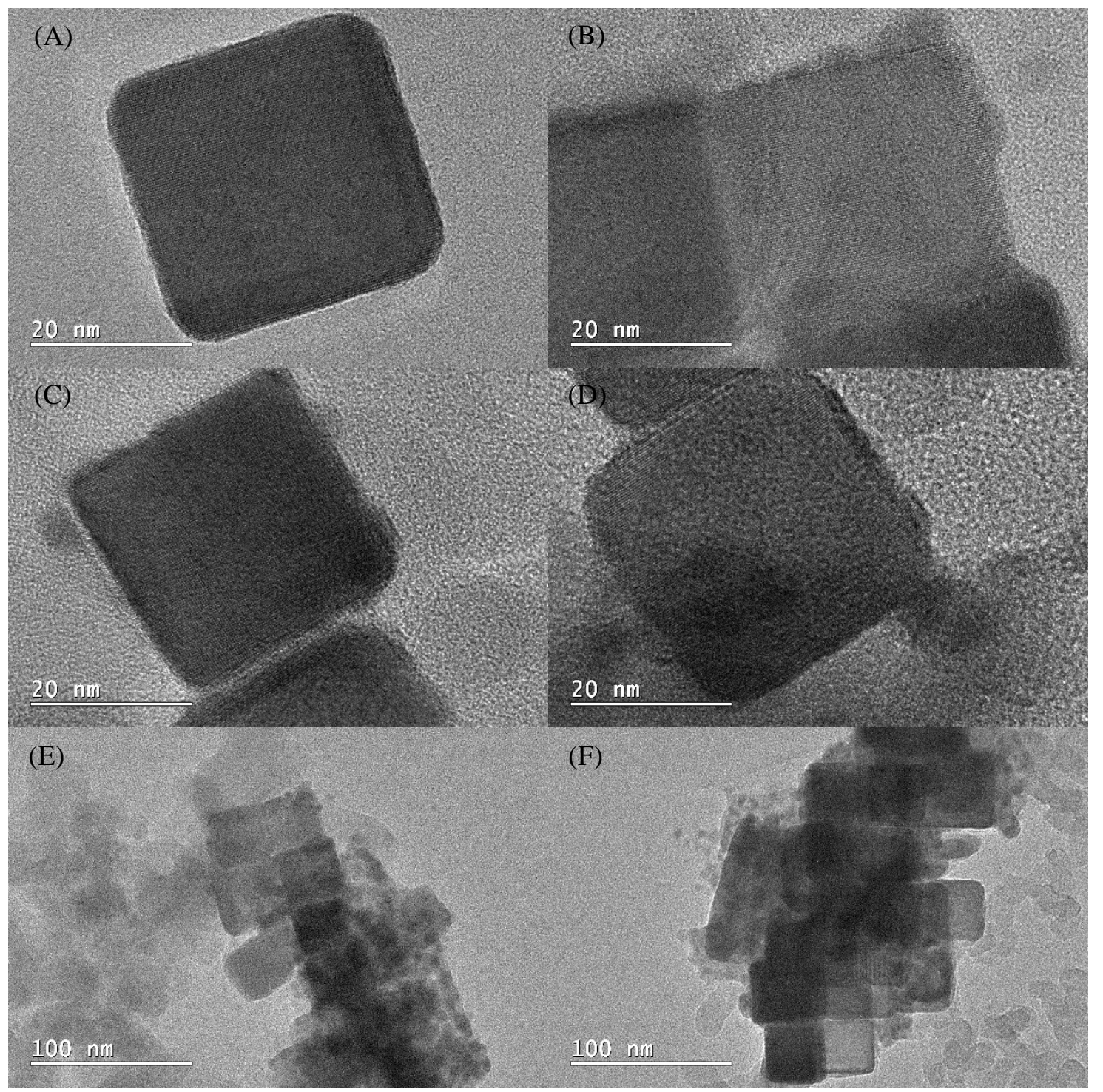

Figure 10f. TEM images of $\mathrm{PbCl}_{2} \mathrm{NCs}$. 
Images of the solution of $\mathrm{PbBr}_{2}$ nanocrystals shows both large irregular shaped particles and clusters of spherical particles (see Figure 10g). These images indicate insufficient stabilisation of the particles to prevent aggregation. However, the particles do display lattice fringing and are therefore crystalline and EDS analysis shows both strong signals from both lead and bromine. To improve the quality of the final product, a higher concentration of ligands could prevent the aggregation of the nanoparticles into larger clusters. Additionally, increasing the ratio of halogen to lead, as is required for $\mathrm{PbCl}_{2} \mathrm{NCs}$, may prove to be beneficial to future attempts at the synthesis of $\mathrm{PbBr}_{2} \mathrm{NCs}$.

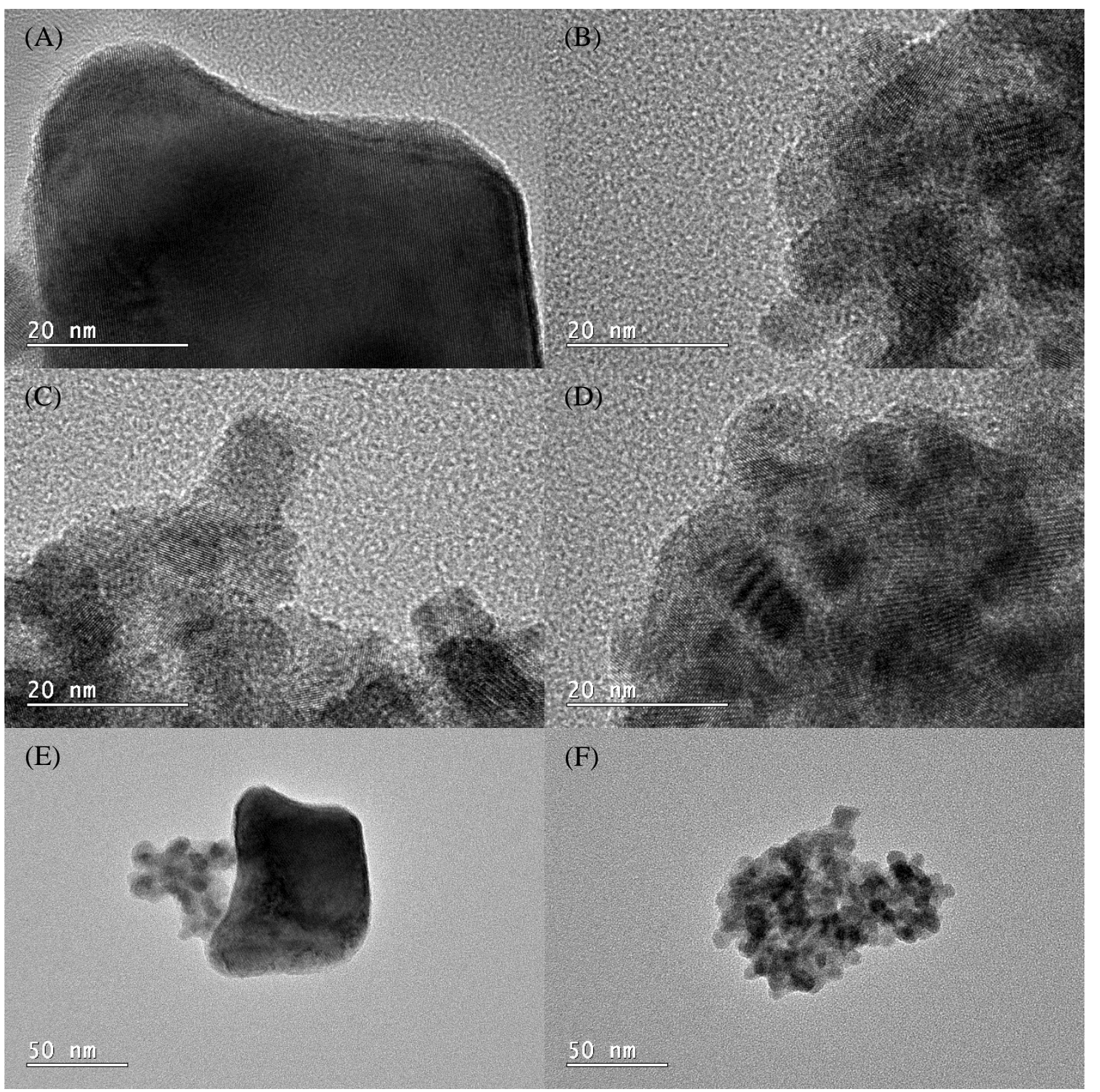

Figure 10g. TEM images of $\mathrm{PbBr}_{2} \mathrm{NCs}$. 
Images of the solution of $\mathrm{PbI}_{2}$ nanocrystals show the sample is composed of $10 \mathrm{~nm}$ cubic particles (see Figure 10h). The particles display weak lattice fringing, indicating a crystalline morphology. The lack of obvious lattice fringing could be explained by the presence of remaining organic material that was not removed during the washing procedure. This material may be coating the particles and obstructing a clear view of them. EDS analysis reveals the composition to be lead and iodine rich, supporting the identification of the product as colloidal $\mathrm{PbI}_{2} \mathrm{NCs}$.

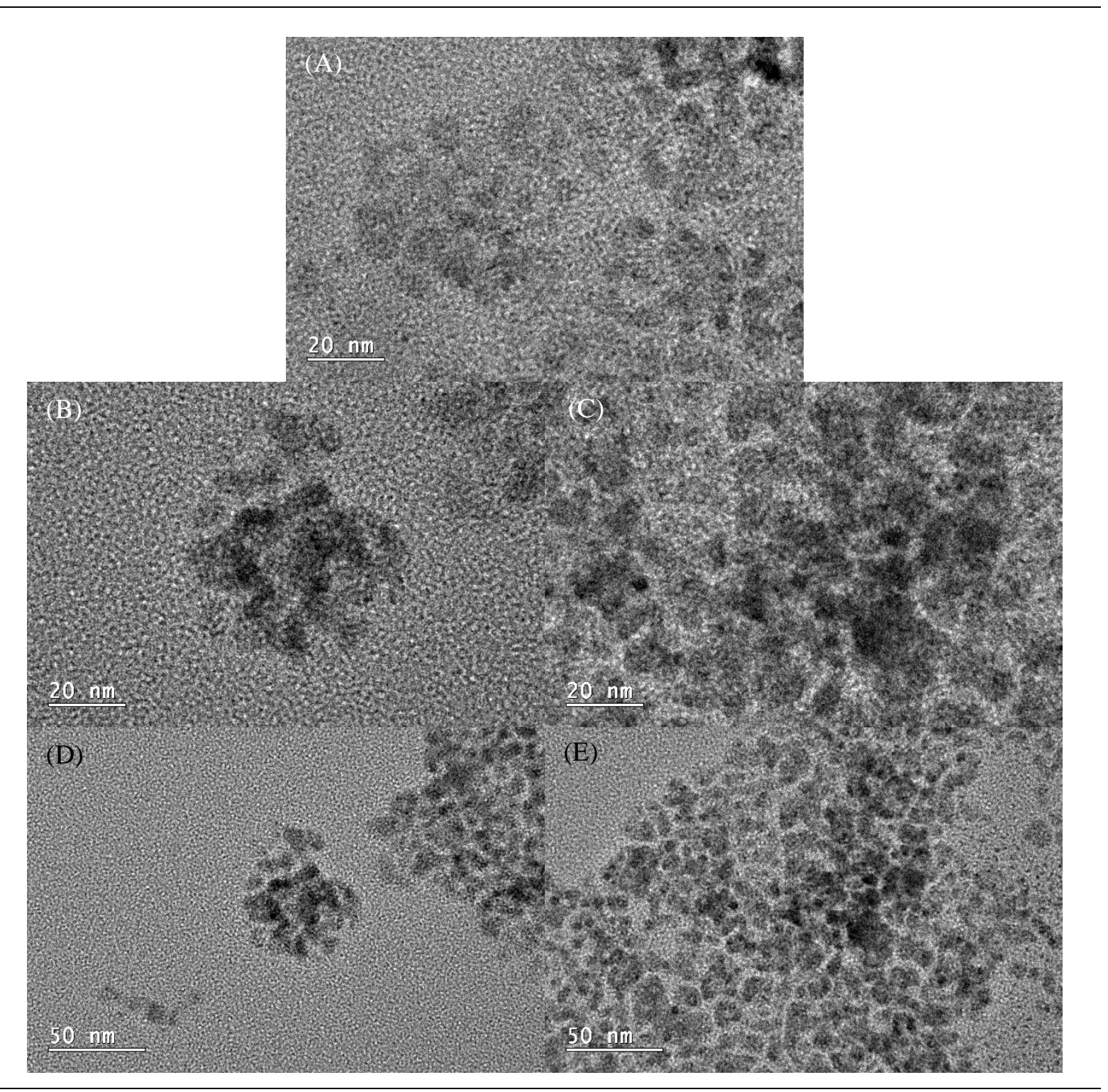

Figure 10h. TEM images of $\mathrm{PbI}_{2} \mathrm{NCs}$. 
10c. Intercalation of (Anthracene-9-Carboxamido)Alkylaminium Halide Salts into Lead Halide Nanocrystals

$1 \mathrm{~mL}$ of lead halide nanocrystal solution is used to suspend two molar equivalents of an (Anthracene9-Carboxamido)Alkylaminium halide (ACAAX) salt in a small vial. The ACAAX salts were made via the same methodology utilised in the synthesis of the thin-film samples, refer to chapter 9 , section 9a. This suspension was allowed to stir for one day to allow for the AACA salt to interact with the $\mathrm{PbX}_{2}$ nanocrystal (see Figure 10i). The vial is then placed in a centrifuge tube and spun at $3800 \mathrm{rpm}$ for 2 mins. Both the solid and the decant is collected and analysed.

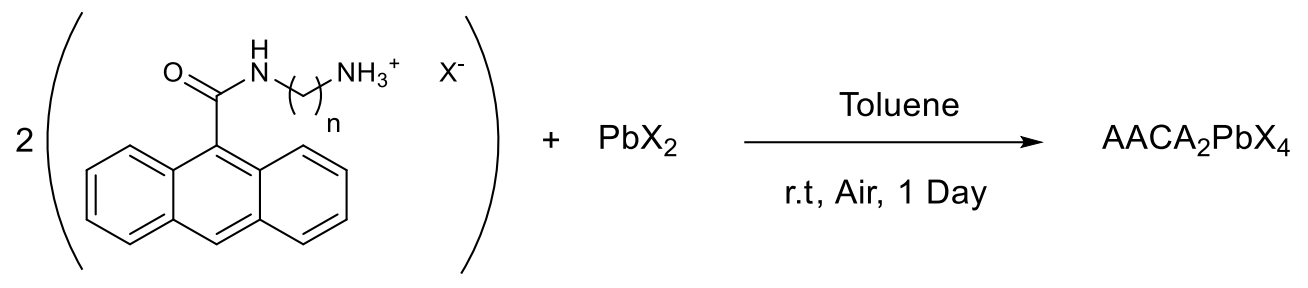

Figure 10i. Formation of $2 D$ ruddlesden-popper perovskite via the intercalation of an ACAA halide salt into lead (II) halide nanoparticles.

\section{$\underline{\text { Analysis }}$}

All samples had their absorption spectrum measured on a Varian Cary 50 Scan UV/Vis Spectrophotometer, their photoluminescence properties including emission, excitation, photoluminescence quantum yield, and emission lifetime measured on an Edinburgh Instruments FLS980 photoluminescence spectrometer, and their particle morphology analysed by either a Joel 2010 transmission electron microscope or a Joel 2100 transmission electron microscope. Note: While $\mathrm{X}$-Ray Diffraction data was desired, the necessary equipment was unavailable during the duration of this project.

\section{0d. Intercalation of (Anthracene-9-Carboxamido)Alkylaminium Halide Salts Results and Discussion}

\section{Observations}

Addition of the (anthracene-9-carboxamido)alkylaminium halide (ACAAX) salt to the lead halide nanocrystal $\left(\mathrm{PbX}_{2} \mathrm{NC}\right)$ solution results in a fine suspension of undissolved ACAAX salt. For lead chloride nanocrystal ( $\left.\mathrm{PbCl}_{2} \mathrm{NCs}\right)$ and lead iodide nanocrystal $\left(\mathrm{PbI}_{2} \mathrm{NCs}\right)$ solutions, the colour of the solution will fade during the course of an hour, indicating the degradation of the nanocrystal suspension. For the iodides, as the colour of the solution fades, the colour of the ACAAX suspension 
will developed a yellow colour, indicating the aggregation of the nanocrystals onto the ACAAX suspension.

\section{Transmission Electron Microscope Images of (Anthracene-9-Carboxamido)Alkylaminium Halide Salts with Lead Halide Nanocrystals}

Analysis of the samples via transmission electron microscopy (TEM) has shown introducing the ACAAX salts to the lead halide nanocrystal solutions results in significant morphological changes to the particles, indicative of significant degradation or aggregation of the nanocrystals which agrees with the decrease in solution colour during the mixing process. For the chlorides, all three samples appear to be dominated by large irregular shaped amalgamations of material (see Figure 10j).
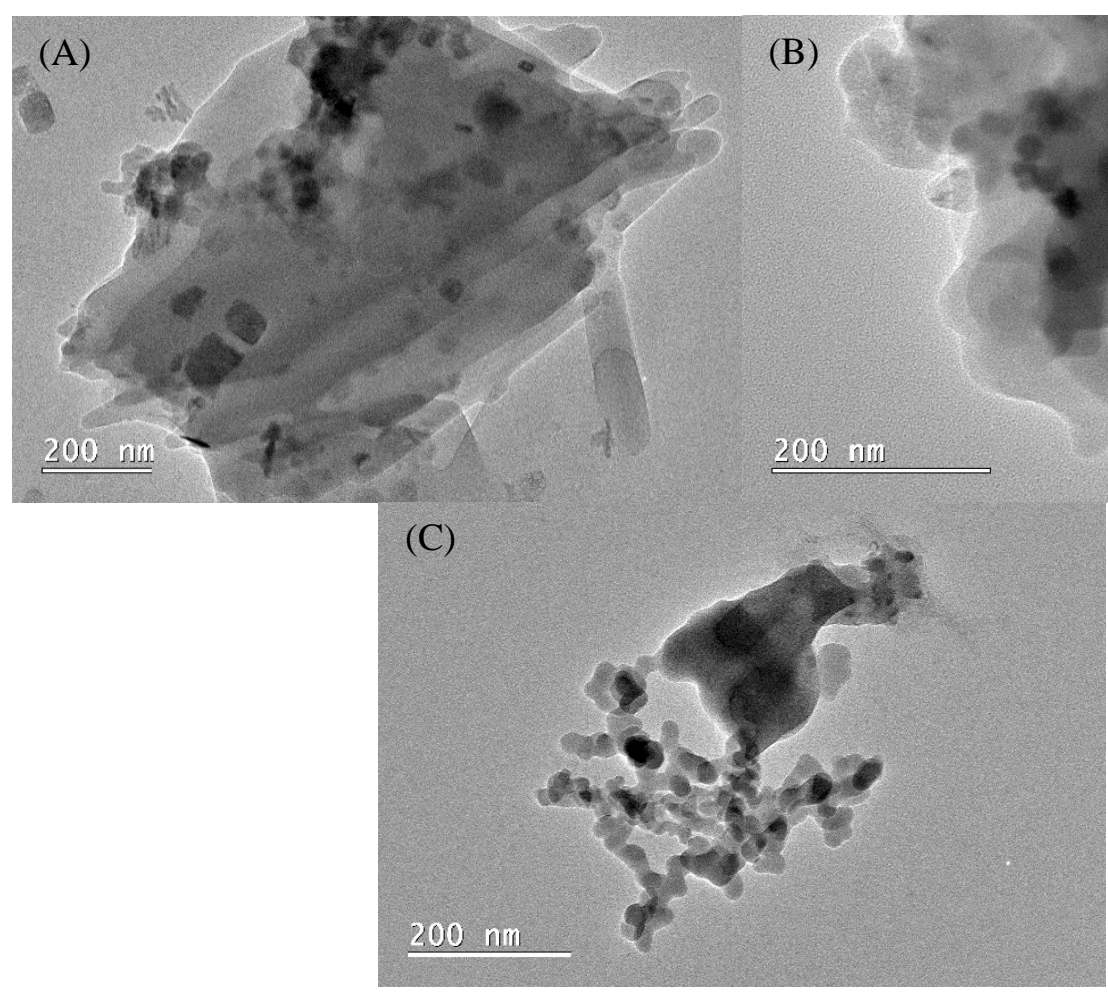

Figure 10j. (A) A TEM image of $\mathrm{PbCl}_{2}+\mathrm{ACEACl}$. (B) A TEM image of $\mathrm{PbCl}_{2}+\mathrm{ACPACl}$. (C) A TEM image of $\mathrm{PbCl}_{2}+\mathrm{ACHpACl}$.

These features tend to be on the scale of hundreds of nanometres to micrometers in size, they lack lattice fringing, and are low density as indicated by the transparency of the features to the electron beam. Energy-dispersive X-ray spectroscopy (EDS) produces a spectrum that is dominated by carbon and oxygen. While the TEM grid that the sample is placed upon contains carbon, the oxygen present coupled with the low density of these features can be used as reliable indicators of organic material i.e. the ACAACl salts. For the samples $\mathrm{PbCl}_{2}+\mathrm{ACEACl}$ and $\mathrm{PbCl}_{2}+\mathrm{ACPACl}$, the large scale features also possess internal components that are distinct from the surrounding material. The much higher density of these internal components suggests the presence of heavier elements, and EDS show 
the presence of chlorine and lead. Therefore, the likely identity of these chloride samples is that they are precipitates of ACAACl salts with the lead chloride nanocrystals imbedded within. Some of these nanoparticles have aggregated with each other, forming large irregular regions of dense material.

The bromides series of samples is very distinct from the earlier chlorides. While all the chloride samples were composed of relatively well preserved nanocrystals imbedded within an organic phase, the bromides are much more variable (see Figure 10k). EDS spectra for the sample $\mathrm{PbBr}_{2}+\mathrm{ACEABr}$ detected the molar percentage of lead within the sample to be less than $1 \%$, suggesting the sample is mostly composed of ACEABr salt. The small amount of lead that was observed would have been associated with degraded nanocrystal material that crashed out with the suspension during the extraction procedure.

The sample $\mathrm{PbBr}_{2}+\mathrm{ACPABr}$ is almost the complete opposite of the $\mathrm{PbBr}_{2}+\mathrm{ACEABr}$ sample, in that the EDS shows this sample contains a significant amount of lead. The sample possesses large, dense shards that are hundreds of nanometres long. Targeted EDS measurements of these features show that lead and bromine dominate their composition. Such a result further supports the ACAAX induced degradation and aggregation of the nanocrystal solutions. Targeted EDS measurements of low density material show high amounts of nitrogen, oxygen, and bromine, but low amounts of lead, indicating that these features are mainly composed of the organic salt. Images such as image (B) in Figure 10k show that there is a significant segregation between the low density and high density material. The images suggest that the $\mathrm{ACPABr}$ salt has facilitated the coalescence of the $\mathrm{PbBr}_{2} \mathrm{NC}$ 's into larger structures but without forming a homogenous phase with it.

For $\mathrm{PbBr}_{2}+\mathrm{ACHpABr}$, EDS spectra of this sample show the significant presence of lead. However, features that possess significant quantities of lead tend to be low in oxygen and bromine, suggesting that like $\mathrm{PbBr}_{2}+\mathrm{ACPABr}$, the degradation of coalescence of $\mathrm{PbBr}_{2} \mathrm{NC}$ 's results in distinct phases of inorganic and organic material. High magnification images of the large scale features within this sample shows significant lattice fringing, indicating a crystalline nature and further supporting the inorganic nature of this sample. 


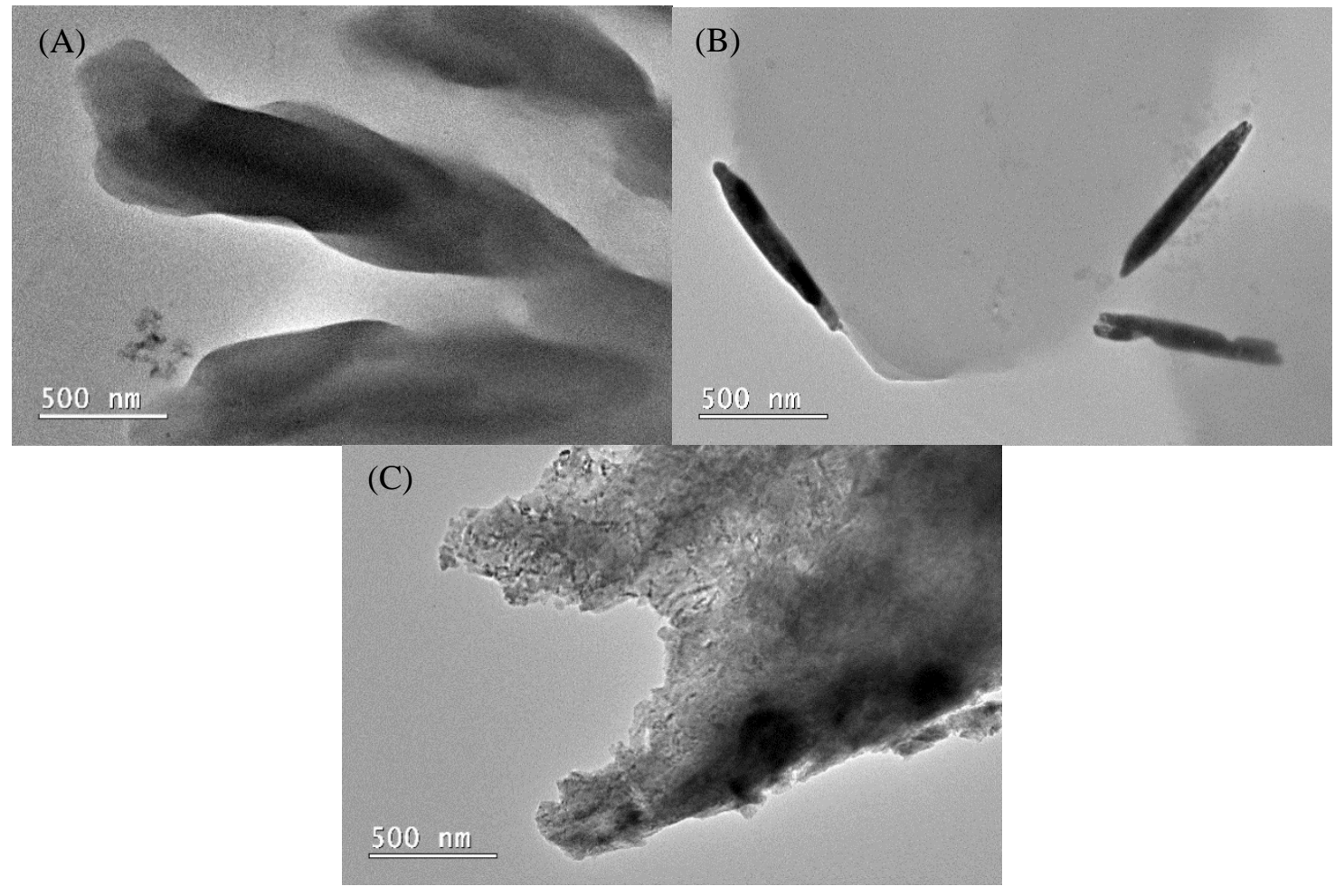

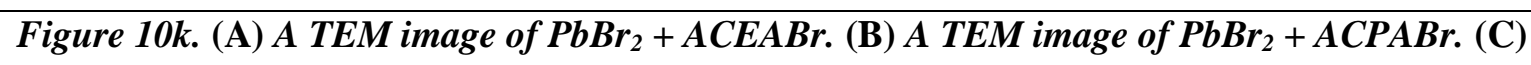
A TEM image of $\mathrm{PbBr}_{2}+\mathrm{ACHpABr}$.

All iodide samples possess significant quantities of lead, iodine, carbon, nitrogen, and oxygen, all within the same structures and therefore indicating a mixed phase of lead iodide and ACAAI salts. The structures present within these samples tend to be large, micrometre scale amalgamations of long rod like features (see Figure 10l). Unique to the sample $\mathrm{PbI}_{2}+\mathrm{ACEAI}$ is the presence of small, cubic particles embedded within a less dense amorphous matrix. Such a structure is similar to the chloride samples $\mathrm{PbCl}_{2}+\mathrm{ACEACl}$ and $\mathrm{PbCl}_{2}+\mathrm{ACPACl}$, though these internal structures are much smaller than those found in the chloride samples, at only $\sim 10 \mathrm{~nm}$ in size. These features are of a comparable size to those of the pure lead iodide nanocrystals ( $\mathrm{PbI}_{2} \mathrm{NC}^{\prime}$ 's), indicating they are $\mathrm{PbI}_{2} \mathrm{NCs}$ that have been incorporated into the amorphous ACEAI phase. However, even at high magnification, only weak lattice fringing could be observed from these features (see Figure $10 l$ image (B)). The lack of lattice fringing could be a result of an amorphous nature to these structures, though, based upon the EDS analysis and the dense nature of these particles, it is possible that these feature are indeed $\mathrm{PbI}_{2}$ and that the lack of lattice fringing is the result of amorphous ACEAI obscuring those signals beyond recognition. $\mathrm{PbI}_{2}+\mathrm{ACPAI}$ and $\mathrm{PbI}_{2}+\mathrm{ACHpAI}$ do not possess any internal structures. The particles present within these samples are long, rod like in shape, and are slightly translucent to the electron beam and are therefore low in density. These rod like structures are accumulated within larger, micrometre scale features. The lack of nanoscale features suggests that the ACPAI and ACHpAI salts are more 


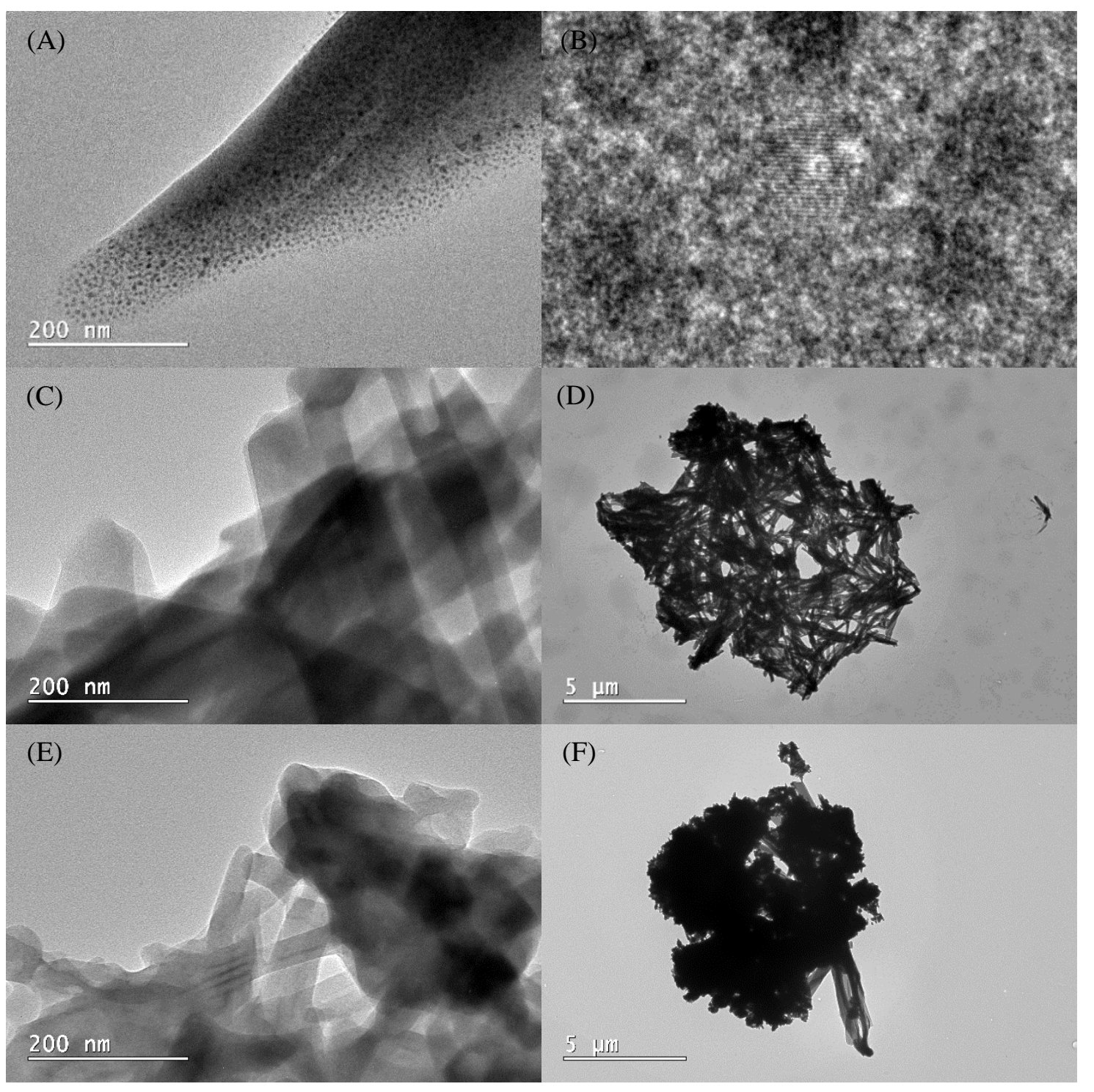

Figure 10l. (A and B) TEM images of PbI ${ }_{2}+$ ACEAI. (C and D) TEM images of PbI $\mathrm{P}_{2}+$ ACPAI. (D and F) TEM images of $\mathrm{PbI}_{2}+A C H p A I$.

effective at interacting and coalescing the nanocrystals than the ACEAI salt, possibly due to the longer alkyl chain. These large scale features are on the same micrometre scale to those in $\mathrm{PbI}_{2}+\mathrm{ACEAI}$. These results show that the lead iodide nanocrystals interact significantly with the ACAAIs salt, resulting in large scale mixed organic/inorganic phases. 


\section{Absorption Measurements of (Anthracene-9-Carboxamido)Alkylaminium Halide Salts with} Lead Halide Nanocrystals

All nine intercalated samples demonstrate high levels of scattering. Comparing the absorption spectra of intercalated samples with those of the pure AAAC solutions shows a clear redshift in peak absorption for all but one of the samples when transitioning from pure solution to mixed phase (see Figure 10m). This redshift is significant enough to induce what appears to be a negative correlation between the two samples absorption spectra. However, a negative correlation would suggest that anthracene absorption is increasing the transparency of the sample which simply cannot be true. Therefore, the best explanation for this apparent negative correlation is that it is a coincidence that has arisen from a significant redshift in the absorption spectrum of the anthracene derivatives due to being incorporated into a mixed phase with the nanocrystals.

The absorption spectrum of $\mathrm{PbCl}_{2}+\mathrm{ACEACl}$ displays two small speaks at $311 \mathrm{~nm}$ and $337 \mathrm{~nm}$. Solutions of pure AEAC display similar peaks at $331 \mathrm{~nm}$ and $346 \mathrm{~nm}$, however these peaks are more significant in terms of their absorption when compared to the equivalent features in $\mathrm{PbCl}_{2}+\mathrm{ACEACl}$. As discussed in Chapter 9, the thin-film samples of $\mathrm{PbCl}_{2}+\mathrm{ACEACl}, \mathrm{PbBr}_{2}+\mathrm{ACEABr}$, and $\mathrm{PbBr}_{2}+\mathrm{ACPABr}$ displayed similar absorption features that did not result in sample emission, suggesting that these absorption bands were inducing photodimerization of anthracene. If the $311 \mathrm{~nm}$ and $337 \mathrm{~nm}$ peaks of the intercalation sample $\mathrm{PbCl}_{2}+\mathrm{ACEACl}$ are also negatively correlated with excitation, then that would suggest that the new mixed phase is one such that reduces the rate of photodimerization. Several mechanisms could reduce the degree at which photodimerization occurs. One is simply that the orientation of anthracene molecules within the mixed phase is not conducive to photodimerization or alternatively, the presence of $\mathrm{PbCl}_{2}$, which absorbs significantly in the 300 to $400 \mathrm{~nm}$ range, may be preventing the photoinduced reaction from taking place by reducing the availability of 300 to $350 \mathrm{~nm}$ light.

In addition to the redshift and drop in absorption at 300 to $350 \mathrm{~nm}$, the mixed phase $\mathrm{PbCl}_{2}+\mathrm{ACEACl}$ do not display an absorption peak at $\sim 420 \mathrm{~nm}$, a peak that is present in the spectrum of pure AEAC solutions. The absence of this peak could be due the presence of $\mathrm{PbCl}_{2}$ or is simply obscured by the significant scattering of light by this sample.

The absorption spectrum of $\mathrm{PbCl}_{2} \mathrm{NCs}$ with $\mathrm{ACPACl}$ is unique in shape. Setting the lowest absorption value to zero and renormalizing the data produces a spectrum that shows a dramatic dichotomy in terms of absorption at wavelengths longer than $400 \mathrm{~nm}$ and those that are shorter. This stands in contrast with the other chloride samples and even the absorption spectrum of pure APCA in which the absorption of such wavelengths is relatively high. The low degree of scattering in this 
(A)

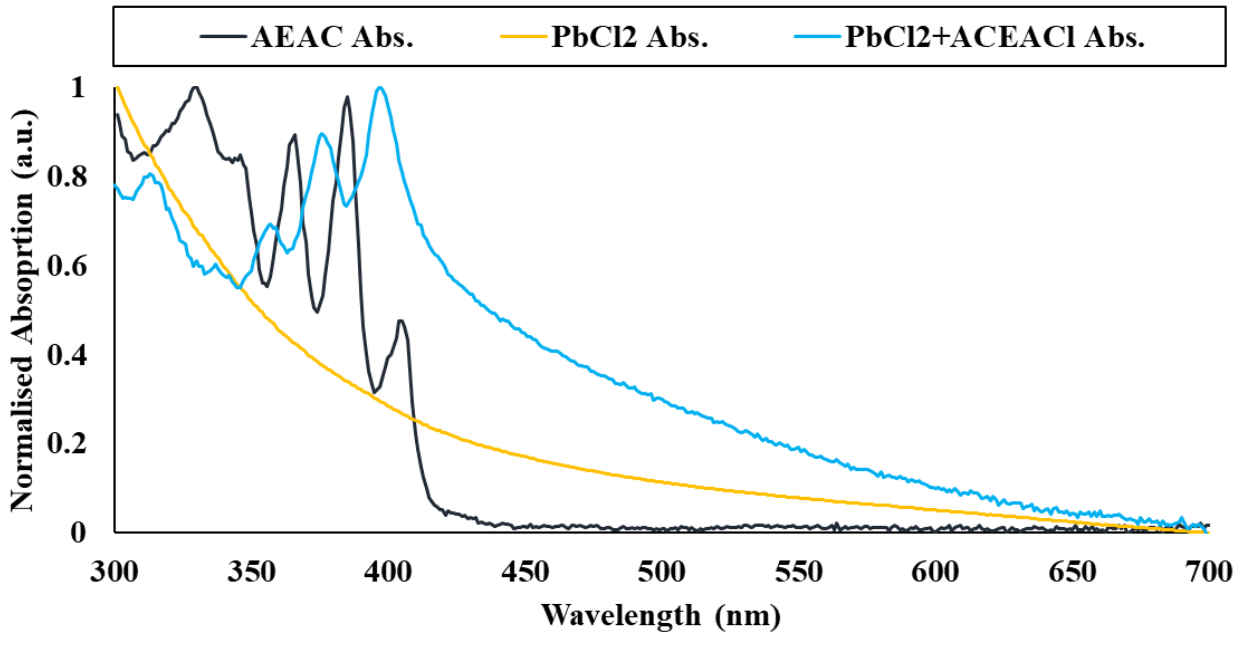

(B)

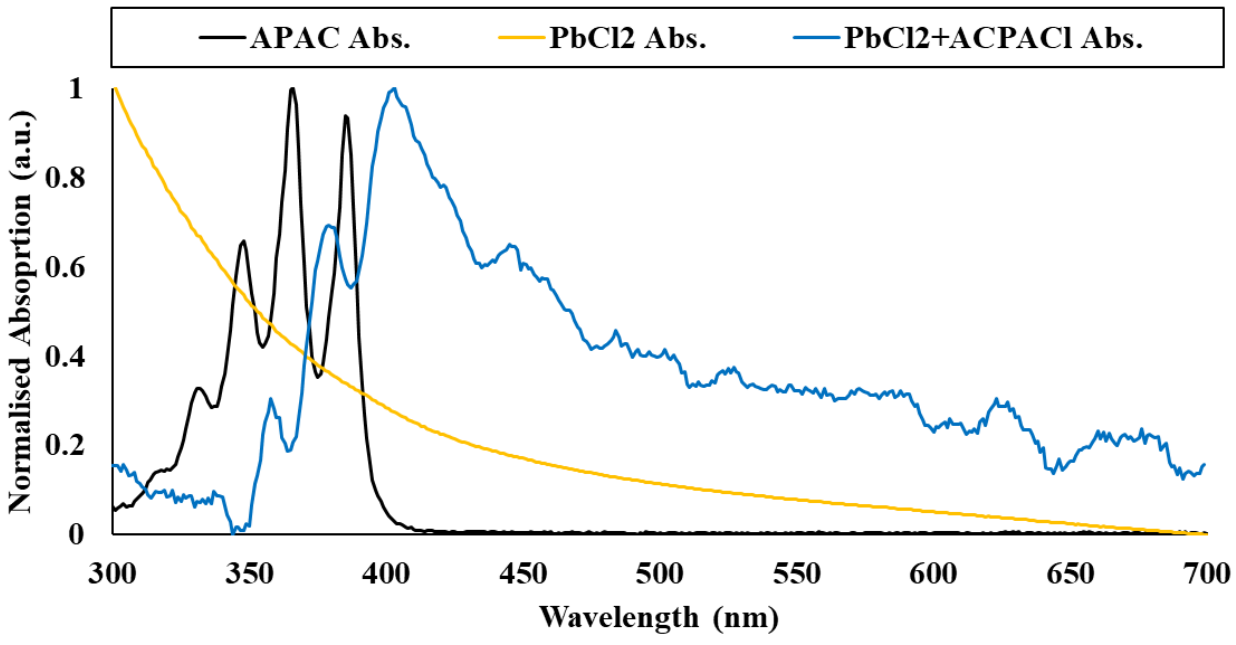

(C)

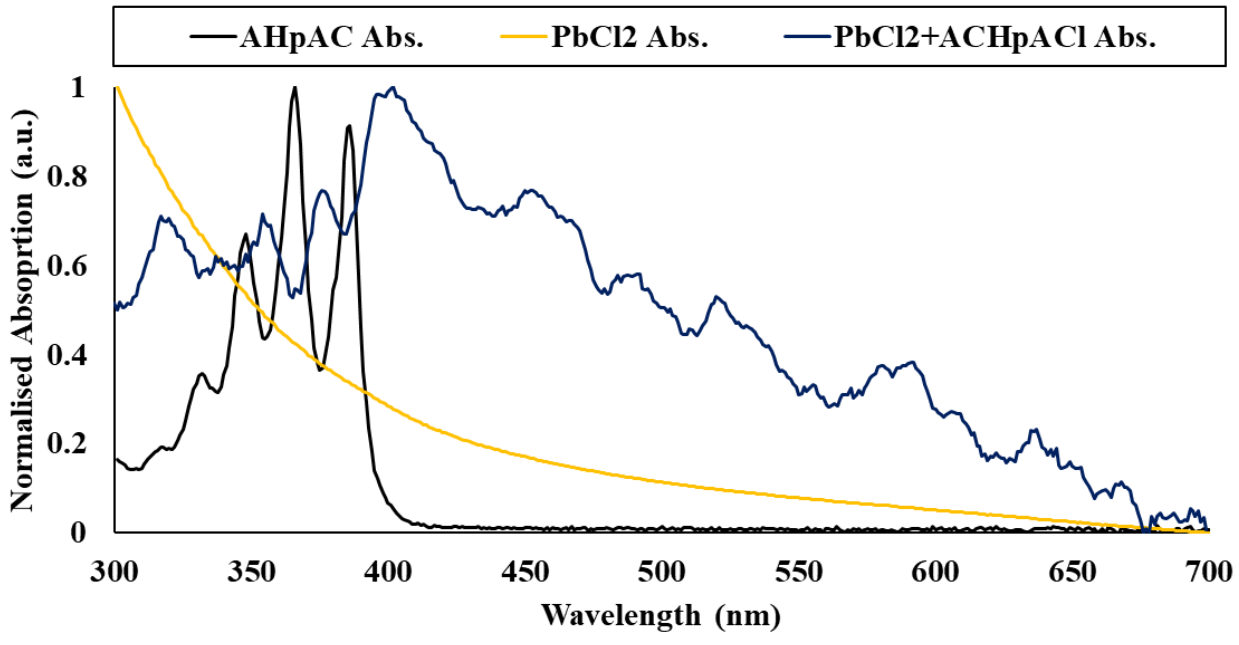

Figure 10m. (A) Comparison between the absorption spectra of $\mathrm{AEAC}, \mathrm{PbCl}_{2}$, and $\mathrm{PbCl}_{2}+$ ACEACl. (B) Comparison between the absorption spectra of $\mathrm{APAC}, \mathrm{PbCl}_{2}$, and $\mathrm{PbCl}_{2}+$ ACPACl. (C) Comparison between the absorption spectra of $\mathrm{AHpAC}, \mathrm{PbCl}_{2}$, and $\mathrm{PbCl}_{2}+$ ACHpACl. 
region of the spectrum may be an indicator that the particles sizes that are present within the sample do not scattering shorter wavelengths and are therefore more transparent to those wavelengths.

Mixing $\mathrm{PbCl}_{2} \mathrm{NCs}$ with $\mathrm{ACHpACl}$ produces an absorption spectrum that lacks definition. However, overlaying the absorption spectra of this sample with those of the other two in the chloride series shows a correlation between absorption maxima, demonstrating that this spectrum possesses the same three prominent peaks (see Figure 10n).

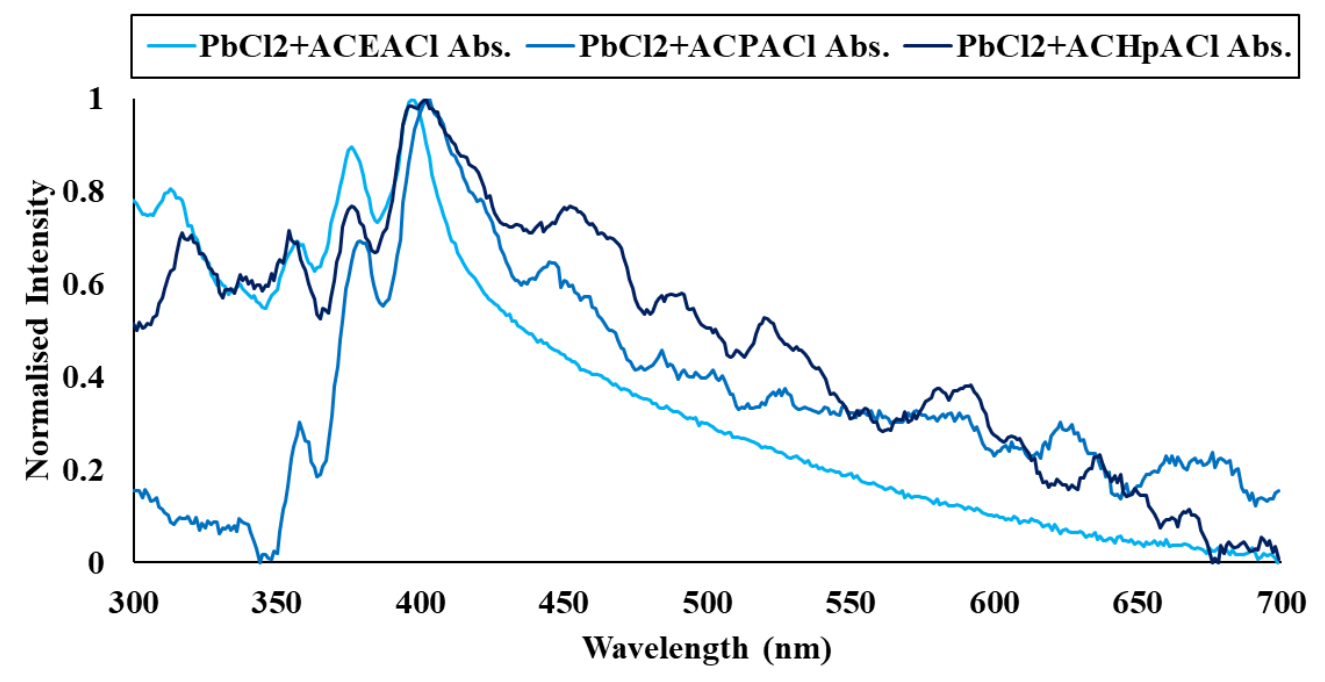

Figure 10n. Overlay of the absorption spectra of $\mathrm{PbCl}_{2}+\mathrm{ACEACl}, \mathrm{PbCl}_{2}+\mathrm{ACPACl}$, $\mathrm{PbCl}_{2}+\mathrm{ACHpACl}$.

The absorption spectra of $\mathrm{PbCl}_{2}+\mathrm{ACHpACl}$ correlates extremely well with that of $\mathrm{PbCl}_{2}+\mathrm{ACEACl}$. The lack of definition in this sample suggests that the relative concentration of $\mathrm{ACHpACl}$ within the sample was low. Such a result correlates well with the TEM images of this samples which showed that the sample was mostly crystalline and rich in lead and chlorine. This may be a consequence of the long heptyl chain of AHpAC, which could have hindered the accumulation of significant quantities of $\mathrm{ACHpACl}$ into a mixed phase with $\mathrm{PbCl}_{2}$.

The bromide samples demonstrate a surprisingly similar series of spectra compared to the chloride samples. Once again, the mixed phase displays a series of peaks that are redshifted with respect to the pure AAAC solutions (see Figure 10o). The mixed phase of $\mathrm{PbBr}_{2} \mathrm{NCs}$ and $\mathrm{ACEABr}$, as with the chloride counterpart, results in the absence in the lowest energy absorption peak at $\sim 420 \mathrm{~nm}$ and a reduction in the absorption of light at wavelengths $\leq 350 \mathrm{~nm}$, however, to a greater degree. The spectrum of this mixed phase is completely lacking the shortest wavelength absorption peak, displaying only one peak at $341 \mathrm{~nm}$. Once again, the incorporation of an AEAC salt into a mixed phase with a head halide has prevented or even eliminated the electronic transitions behind these absorption features. 
(A)

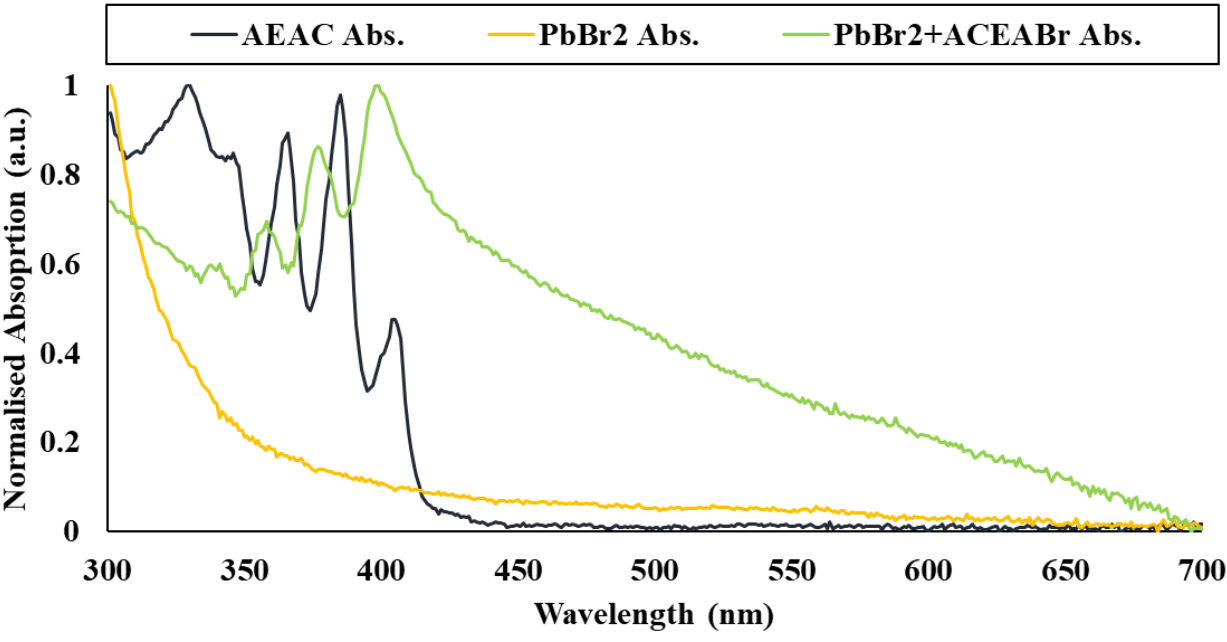

(B)

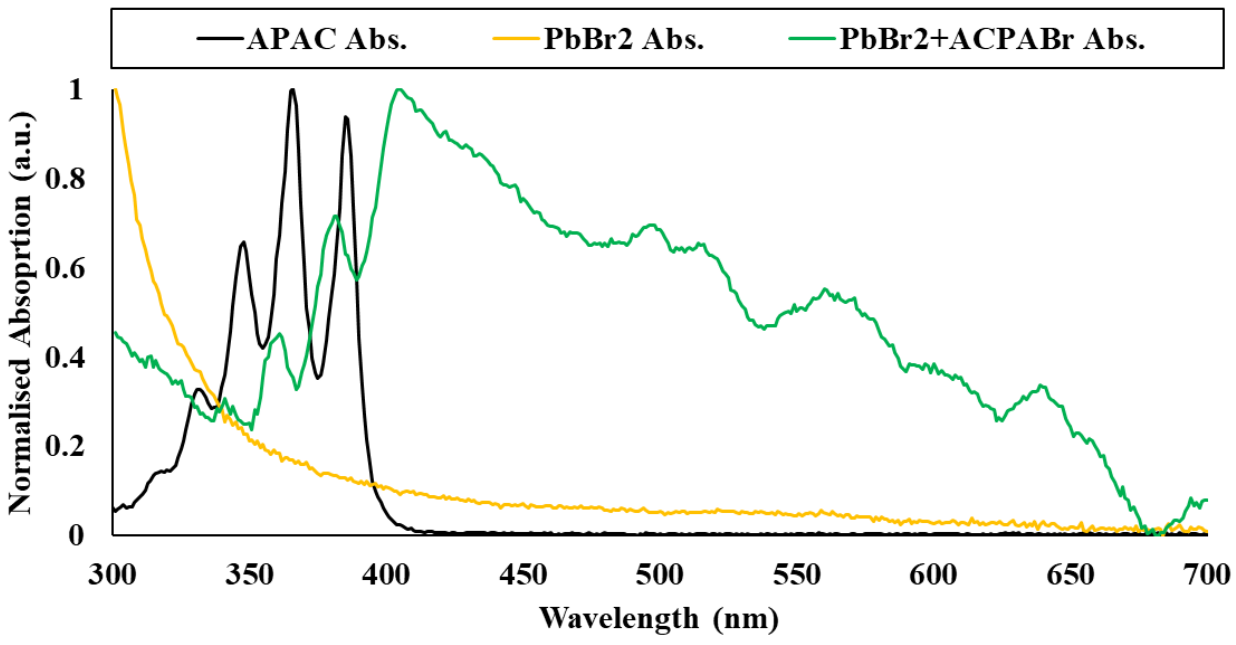

(C)

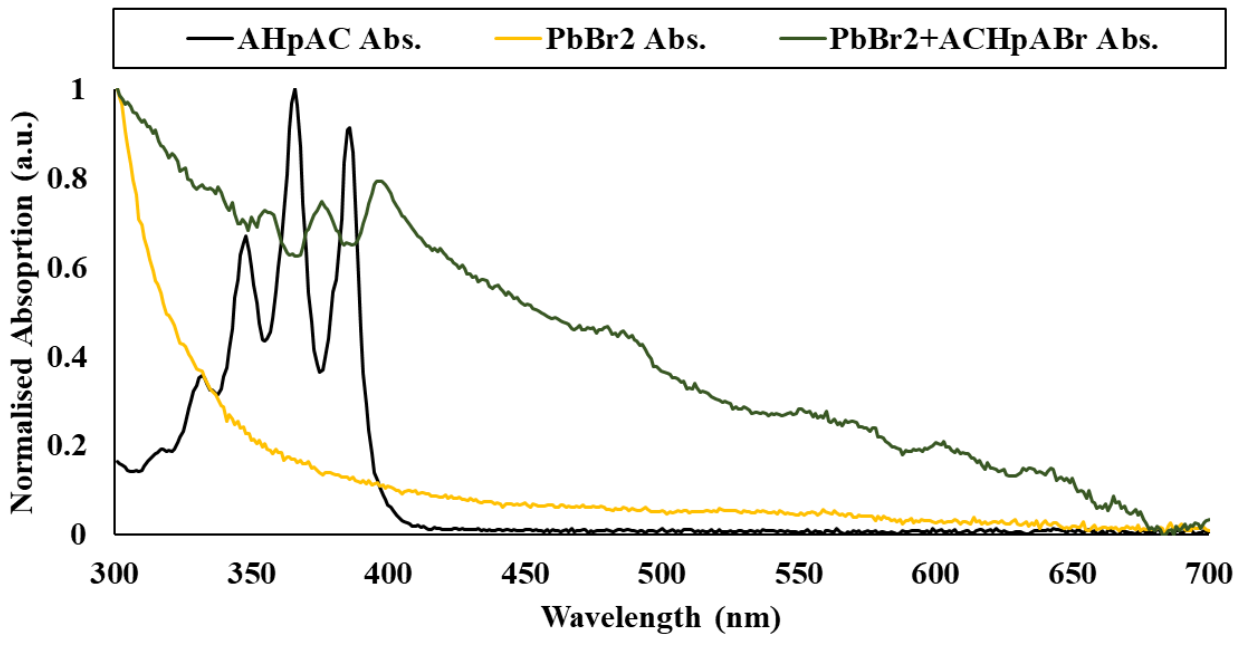

Figure 10o. (A) Comparison between the absorption spectra of $\mathrm{AEAC}, \mathrm{PbBr}_{2}$, and $\mathrm{PbBr}_{2}+$ ACEABr. (B) Comparison between the absorption spectra of APAC, $\mathrm{PbBr}_{2}$, and $\mathrm{PbBr}_{2}+$ ACPABr. (C) Comparison between the absorption spectra of $\mathrm{AHpAC}, \mathrm{PbBr}_{2}$, and $\mathrm{PbBr}_{2}+$ ACHpABr. 
Another similarity between this series of samples and the chlorides is the unique spectrum that is produced by the sample containing ACPAX salt. Once again, this anthracene derivative mixed with the lead halide nanocrystals produces a mixed phase that displays a dramatic reduction in absorption at wavelengths shorter than $400 \mathrm{~nm}$. While the absorption in this region isn't as relatively low as seen for the sample $\mathrm{PbCl}_{2}+\mathrm{ACPACl}$, it does display the same behaviour, suggesting a unique, shared property of $\mathrm{PbX}_{2}+\mathrm{ACPAX}$ mixed phases.

A notable difference between the bromide series and the chloride series is in the absorption spectrum of the samples containing AHpAC. Compared to the chloride samples, the bromide equivalent displays an absorption spectrum that is much more well defined and displays a less significant degree of scattering in the absorption spectra between 300 and $350 \mathrm{~nm}$. However, Overlaying the spectra of all three samples shows good correlation between the absorption peaks (see Figure 10p).

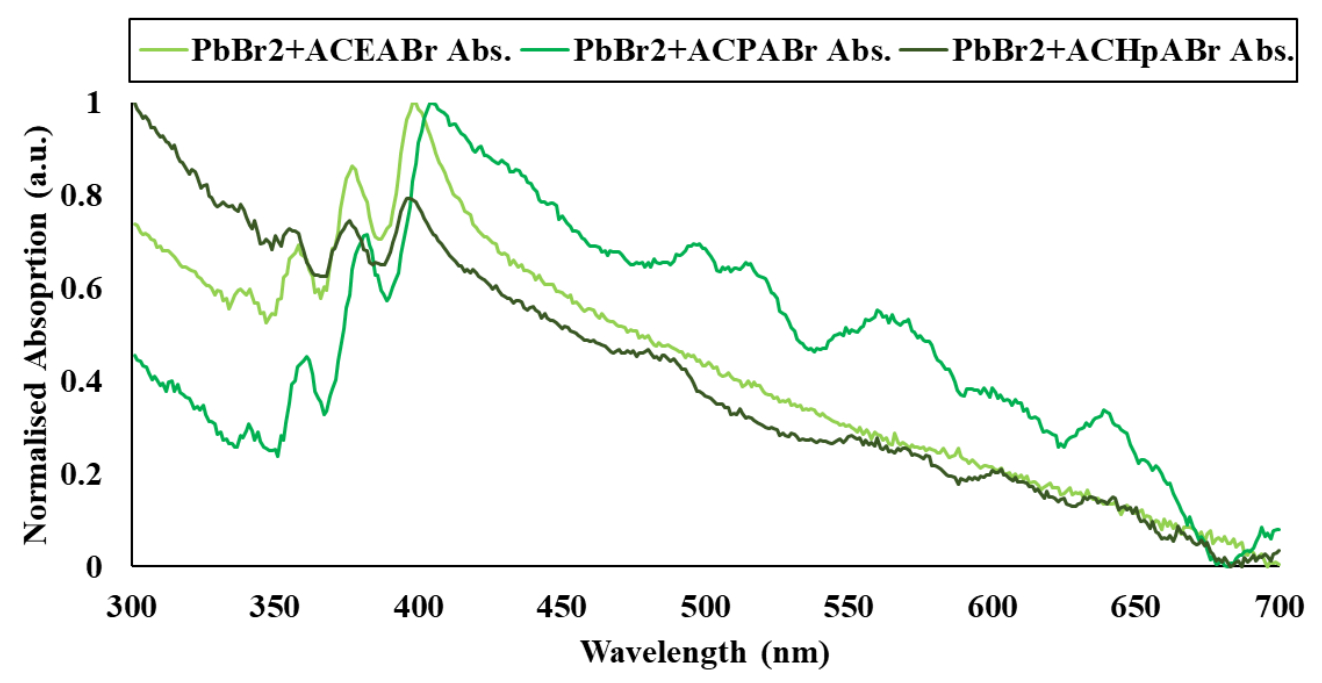

Figure 10p. Overlay of the absorption spectra of $\mathrm{PbBr}_{2}+\mathrm{ACEABr}, \mathrm{PbBr}_{2}+\mathrm{ACPABr}$, $\mathrm{PbBr}_{2}+\mathrm{ACHpABr}$.

The iodide series displays unique trends when compared to the other two halide series (see Figure 10q). Firstly, no redshift of the absorption bands is observed between pure AEAC solutions and the mixed phase of ACEAI and lead iodide $\left(\mathrm{PbI}_{2}\right)$. However, there is still a lack of an absorption band at around 300 to $350 \mathrm{~nm}$, and there is no well-defined peak at $\sim 420 \mathrm{~nm}$. Therefore, the mechanism that drives the redshift is independent to the process that eliminates the unique absorption bands of pure AEAC solutions. While the last sample did not display a redshift, the other two iodide samples did. Therefore, the interaction with the $\mathrm{PbX}_{2}$ nanocrystals that has resulted in the redshift of the other eight samples appears to have not occurred in the $\mathrm{PbI}_{2}+\mathrm{ACEAI}$. This lack of a redshift could be attributed to the unique morphology of $\mathrm{PbI}_{2}+\mathrm{ACEAI}$, which is composed of inorganic $\mathrm{PbI}_{2} \mathrm{NCs}$ imbedded within an amorphous organic matrix. 
(A)

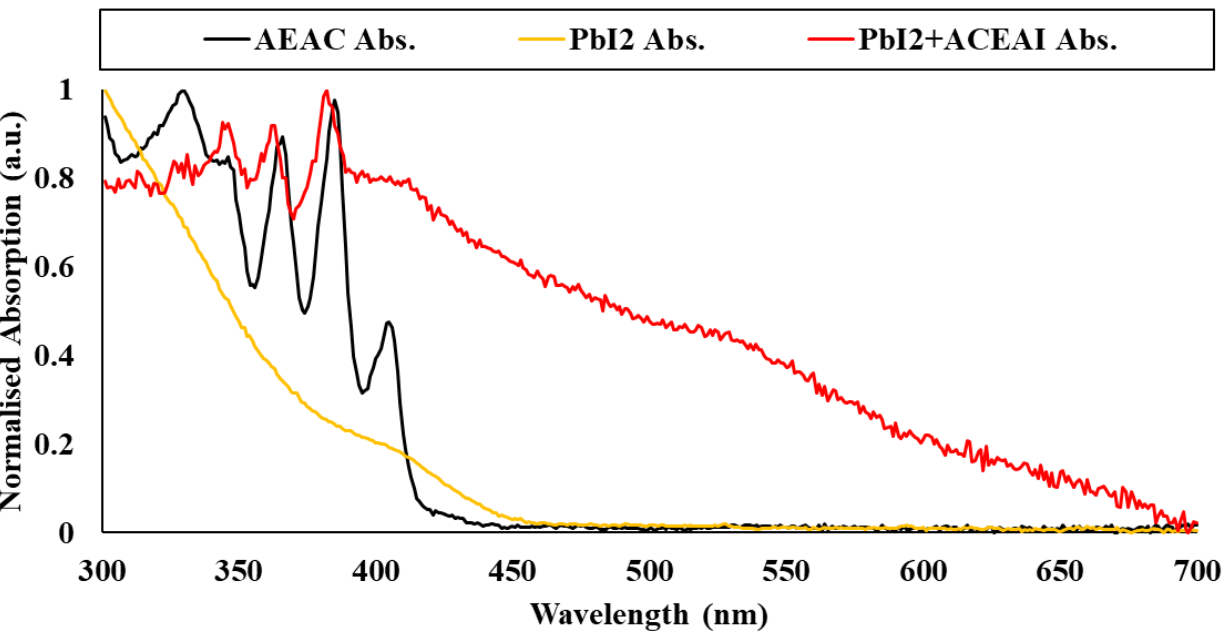

(B)

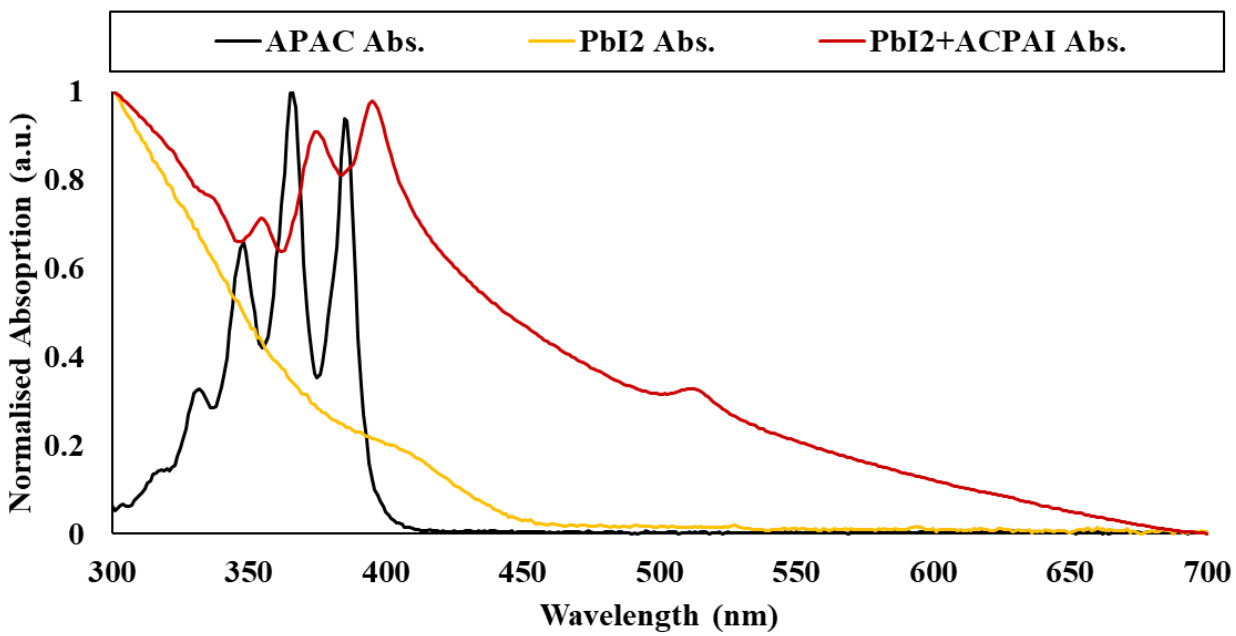

(C)

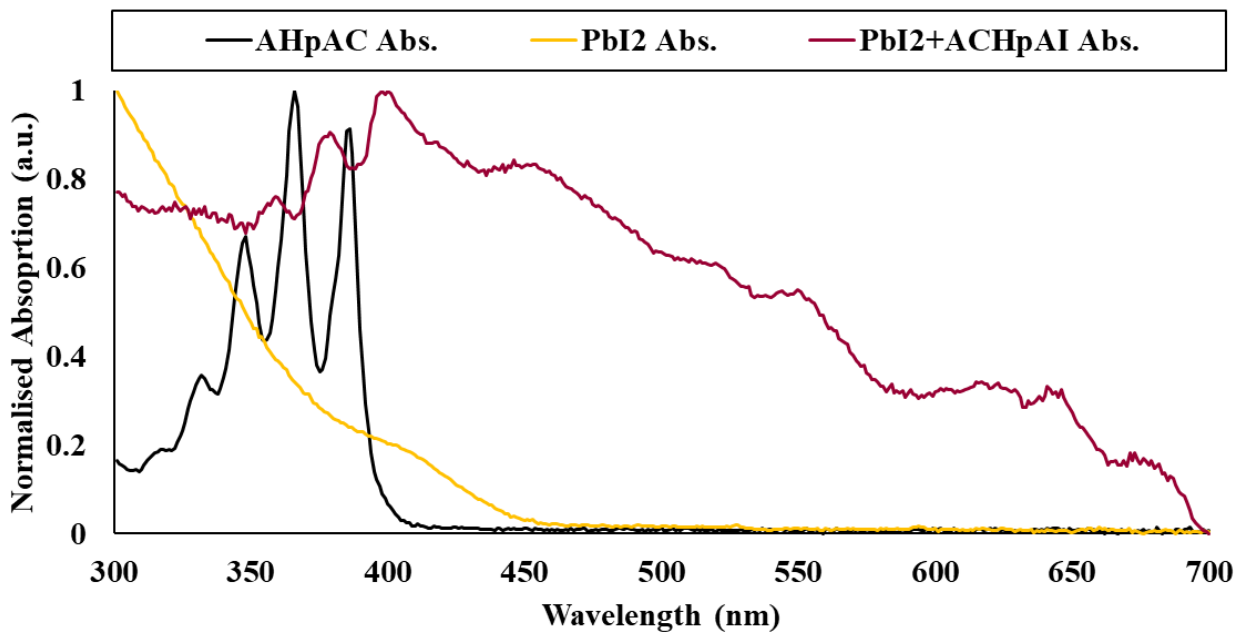

Figure 10q. (A) Comparison between the absorption spectra of AEAC, $\mathrm{PbI}_{2}$, and $\mathrm{PbI}_{2}+\mathrm{ACEAI}$.

(B) Comparison between the absorption spectra of APAC, $\mathrm{PbI}_{2}$, and $\mathrm{PbI}_{2}+\mathrm{ACPAI}$.

(C) Comparison between the absorption spectra of AHpAC, $\mathrm{PbI}_{2}$, and $\mathrm{PbI}_{2}+\mathrm{ACHpAI}$. 
One trend that is shared among all three halide series is the reduction in scattering at wavelengths less than $400 \mathrm{~nm}$ by samples containing APAC. However, while both the chloride and the bromide samples maintain a similar level of scattering after the drop till the end of the scan at $300 \mathrm{~nm}$, such a pattern does not occur for $\mathrm{PbI}_{2}+\mathrm{ACPAI}$. For the iodide sample once a minimum is reached, the absorption rises again continuously. This rise in absorption could be attributed to the presence of $\mathrm{PbI}_{2}$, which would have raised the absorption of light in this spectral region significantly above those of the equivalent samples in the other halide series. However, such an absorption band would have been expected in the other iodide samples.

The last sample, $\mathrm{PbI}_{2}+\mathrm{ACHpAI}$, displays an absorption spectrum that demonstrates the redshift of $\mathrm{PbI}_{2}+\mathrm{ACPAI}$ but lacks the continuous rise in scattering from 350 to $300 \mathrm{~nm}$. Comparing all three iodide samples shows that the sample $\mathrm{PbI}_{2}+\mathrm{ACPAI}$ possesses a high concentration of ACPAI molecules, while the other two iodide samples demonstrate relatively low anthracene absorption features, indicating low AEAC/AHpAC concentration (see Figure 10r).

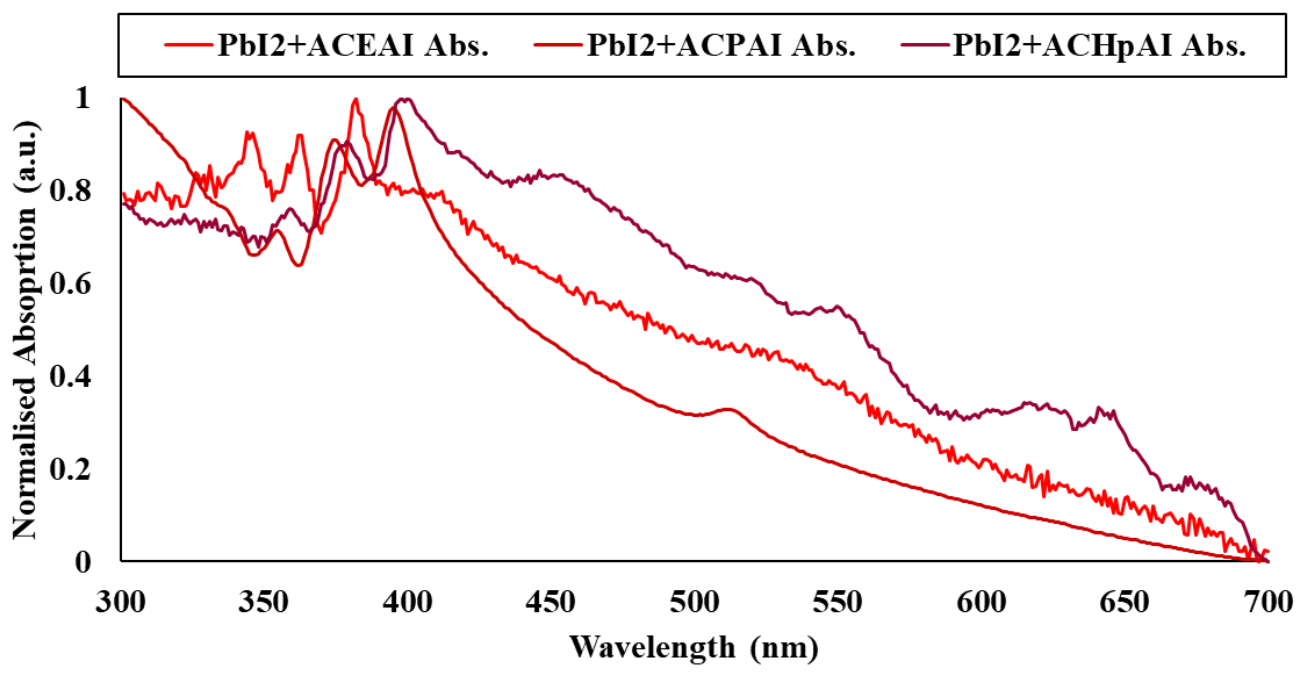

Figure 10r. Overlay of all three absorption spectra of the iodide series of intercalated samples.

Referring to the sample $\mathrm{PbI}_{2}+\mathrm{ACPAI}$, the spectrum of this sample is uniquely noise free when compared to all the other iodide samples. Why this particular sample demonstrates a clear and well defined absorption spectrum and the other samples do not is unknown, but it may be related to a more purified sample, however, all nine samples were processed in the same way. In addition, the sample displays a clear additional peak at $\sim 510 \mathrm{~nm}$. This peak may be the result of a new electronic transition, possibly an excitonic feature as it is in an approximately appropriate region for a two-dimensional lead iodide perovskite, which is typically $517 \mathrm{~nm} .^{53}$ 


\section{Photoluminescence of (Anthracene-9-Carboxamido)Alkylaminium Halide Salts with Lead Halide Nanocrystals}

The electronic emission spectrum of all nine samples strongly mirrors that of the pure AAAC solutions (see Figure 10s, 10t, and 10u). Besides $\mathrm{PbCl}_{2}+\mathrm{ACHpACl}, \mathrm{PbBr}_{2}+\mathrm{ACPACl}$, all samples demonstrate a slight redshift in the peak emission wavelength when compared to the equivalent AAAC.

All samples possessing AEAC demonstrate an emission spectrum that lacks the definition of the pure solution. For example, the electronic emission spectrum of AEAC possesses three well defined peaks, while neither AEAC possessing intercalated samples demonstrate such peaks. $\mathrm{PbCl}_{2}+\mathrm{ACEACl}$ and $\mathrm{PbBr}_{2}+\mathrm{ACEABr}$ produce highly similar spectra, with a peak emission wavelength redshifted with respect to AEAC of $\sim 15 \mathrm{~nm}$ and a prominent shoulder peak at $\sim 450 \mathrm{~nm}$. The only real difference is a second shoulder peak that is only shown by $\mathrm{PbBr}_{2}+\mathrm{ACEABr}$.

While pure solutions of AEAC and APAC demonstrate almost identical emission spectra, the sample $\mathrm{PbBr}_{2}+\mathrm{ACEABr}$ and $\mathrm{PbBr}_{2}+\mathrm{ACPABr}$ are quite distinct from each other. While the former demonstrates a redshifted and non-well defined emission spectrum, the latter is not redshifted and demonstrates three well defined peaks, suggestive of a lack of reabsorption and/or excimer emission.

Another sample that demonstrates a non-redshifted emission spectrum is $\mathrm{PbCl}_{2}+\mathrm{AHpAC}$, unlike the bromide equivalent $\mathrm{PbBr}_{2}+\mathrm{ACHpABr}$ which does. The former produces an electronic emission spectrum with two well defined peaks of equal intensity and a prominent shoulder peak, while the spectrum of the latter demonstrates one peak, and two shoulder peaks. Since there is no common factor between $\mathrm{PbCl}_{2}+\mathrm{ACHpACl}$ and $\mathrm{PbBr}+\mathrm{ACPABr}$, explanations for this seemly random observation includes a different mixed phase compared to the other seven samples, a mixed phase never formed, the mixed phase has degraded, or that the concentration of these samples was lower and therefore they exhibit reduced reabsorption events and excimer formation.

Overall, neither the chloride nor bromide series demonstrate any noticeable trends in terms of emission that can be related to alkyl chain length or halogen present. The same can be said for the iodide samples, however, while the chloride and bromides demonstrated great variability, all three iodides are highly similar to each other. $\mathrm{PbI}_{2}+\mathrm{ACEAI}$ and $\mathrm{PbI}_{2}+\mathrm{ACPAI}$ display almost identical spectra, while $\mathrm{PbI}_{2}+\mathrm{ACHpAI}$, which had a higher signal to noise ratio than the other two iodide samples, demonstrates a similar redshift in peak emission wavelength. Therefore, based upon the emission spectra, no trends can be attributed to halogen or alkyl chain length, and all nine samples display anthracene emission of some kind. 
(A)

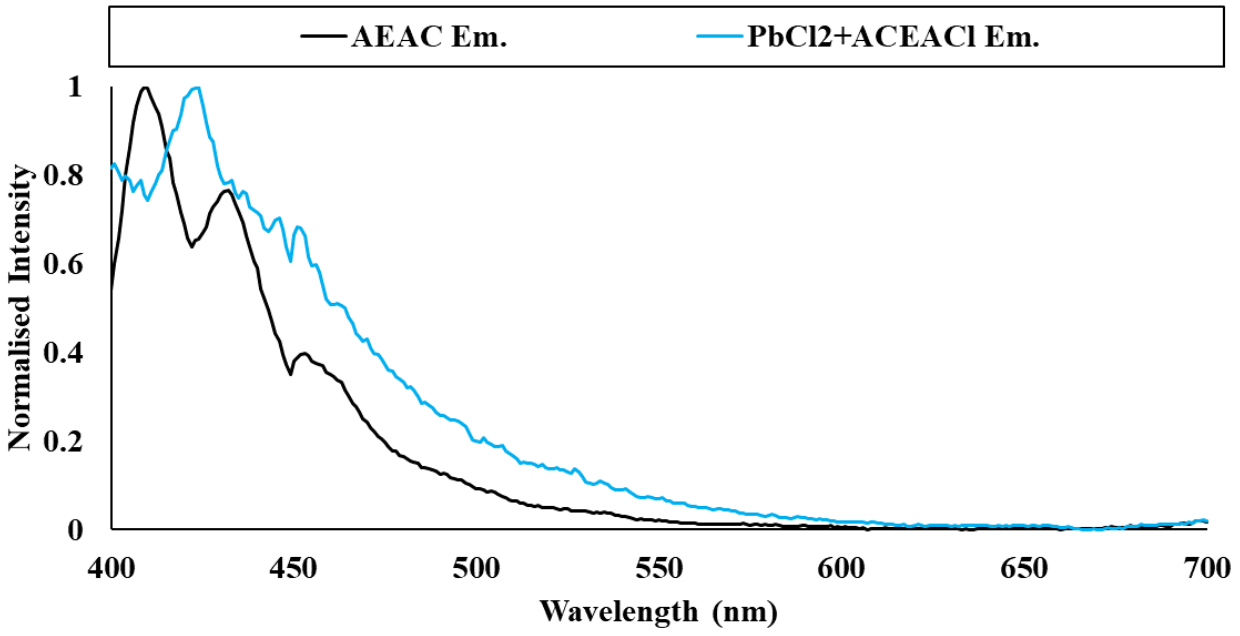

(B)

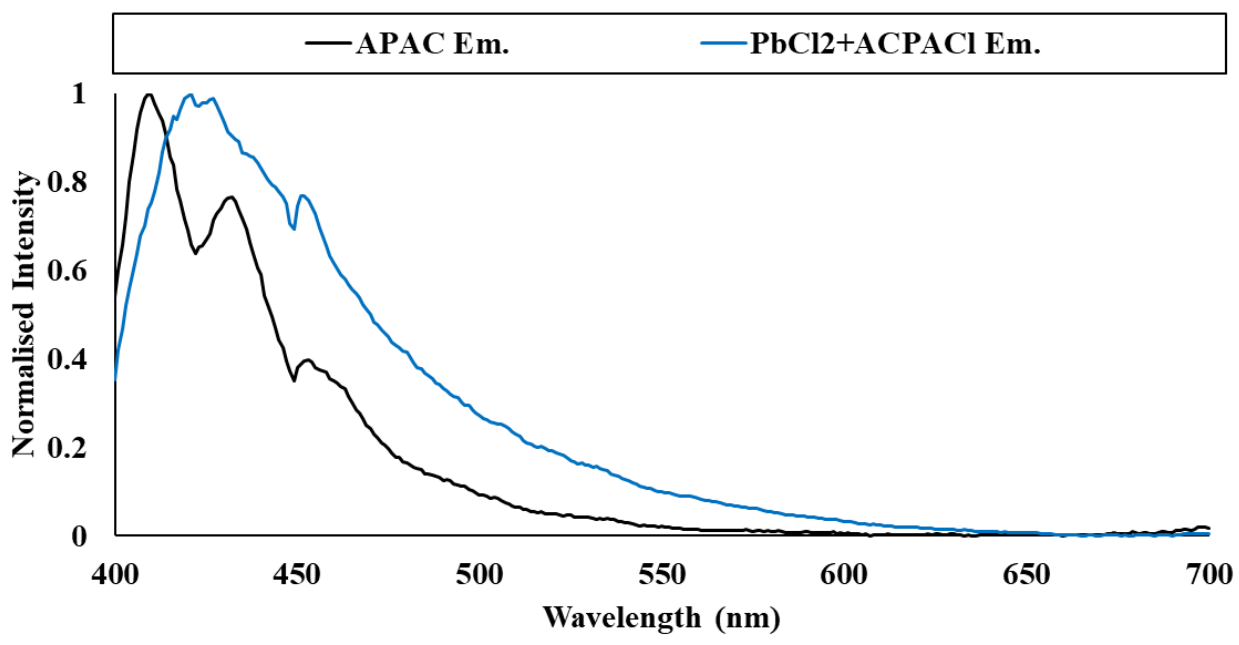

(C)

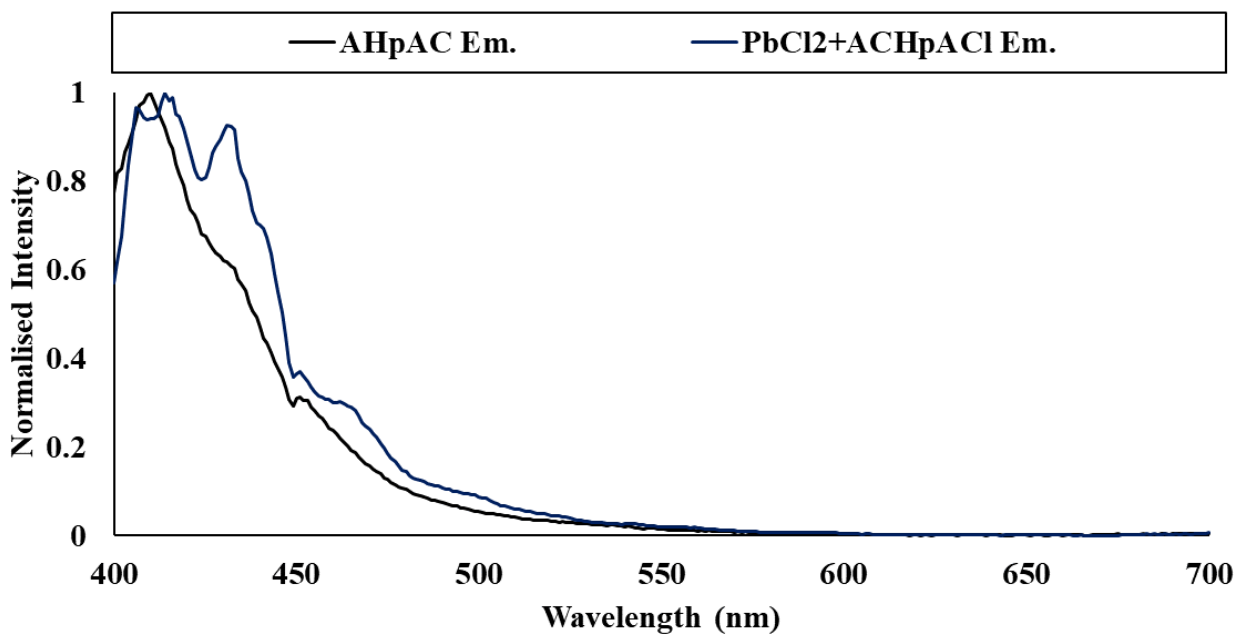

Figure 10s. (A) Comparison between the emission spectra of AEAC, and $\mathrm{PbCl}_{2}+\mathrm{ACEACl}$.

(B) Comparison between the emission spectra of APAC, and PbCl + ACPACl. (C) Comparison between the emission spectra of $\mathrm{AHpAC}$, and $\mathrm{PbCl}_{2}+\mathrm{ACHpACl}$. 
(A)

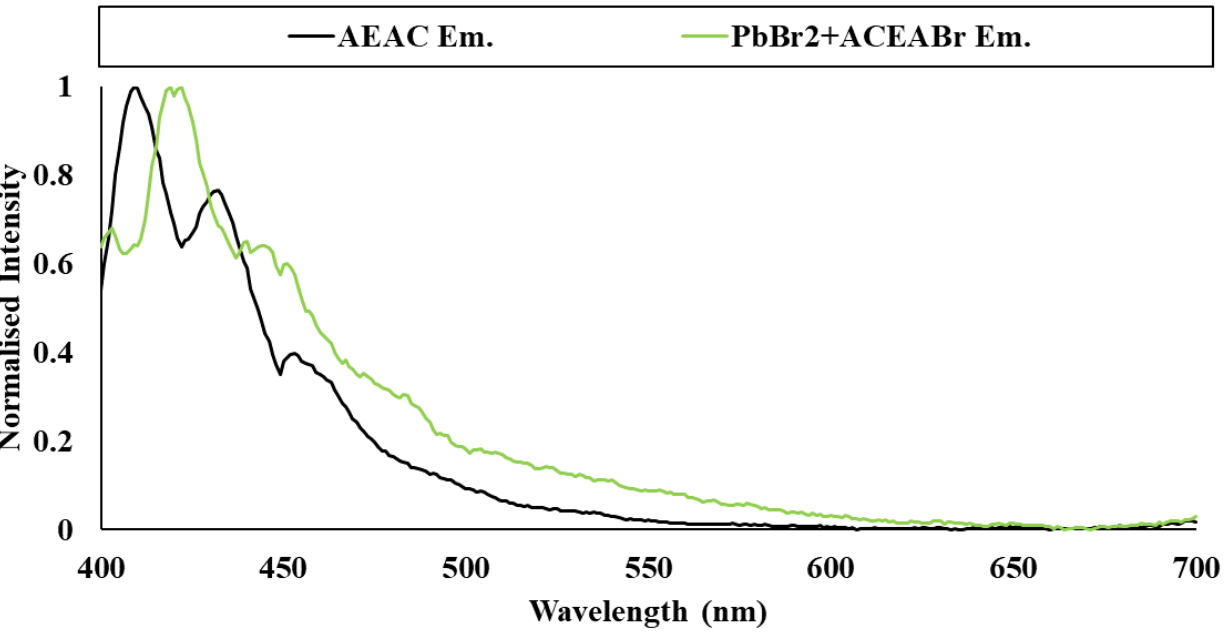

(B)

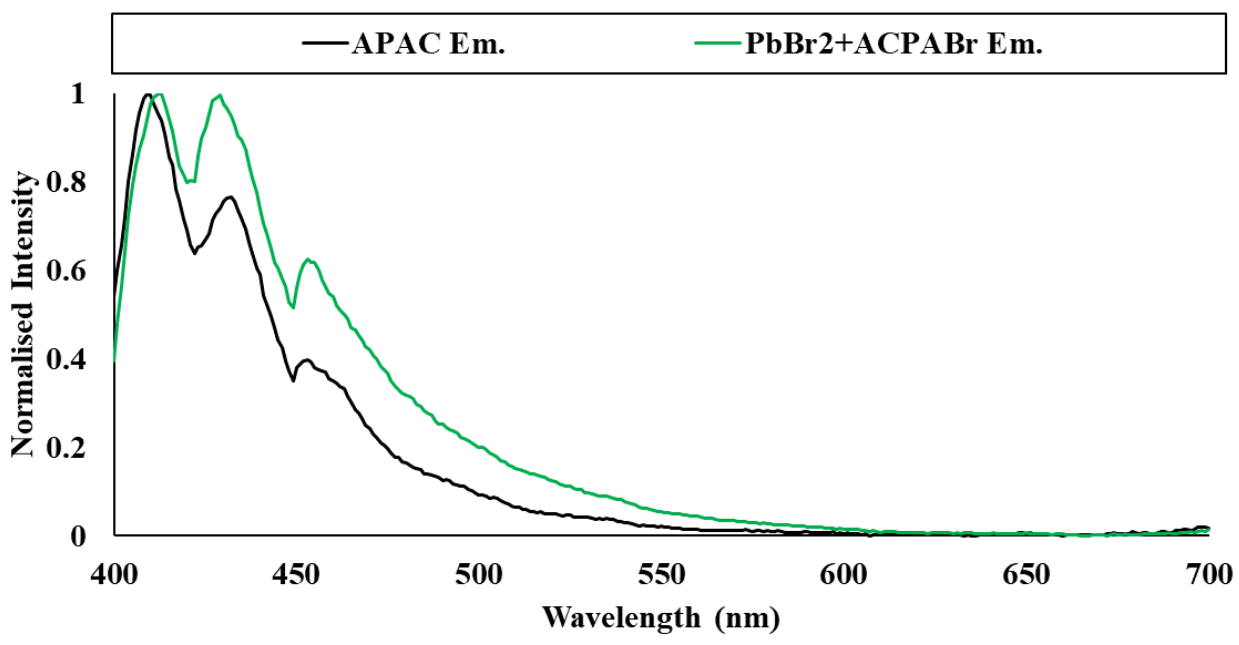

(C)

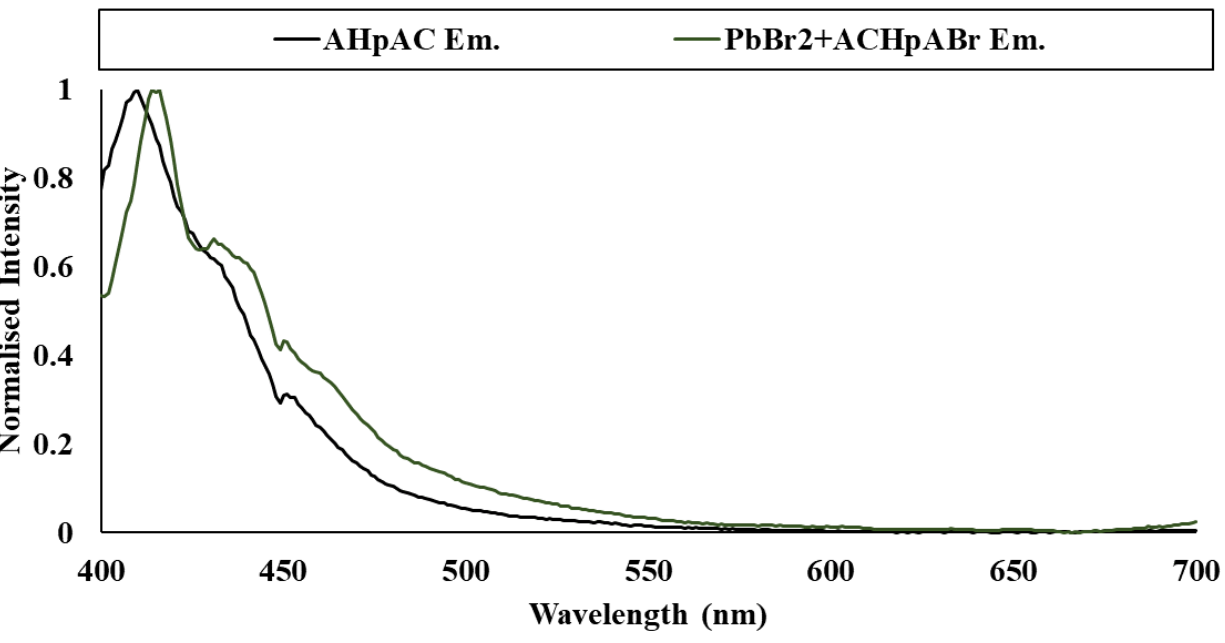

Figure 10t. (A) Comparison between the emission spectra of AEAC, and $\mathrm{PbBr}_{2}+\mathrm{ACEABr}$.

(B) Comparison between the emission spectra of APAC, and PbBr $r_{2}+A C P A B r$. (C) Comparison between the emission spectra of AHpAC, and $\mathrm{PbBr}_{2}+\mathrm{ACHpABr}$. 
(A)

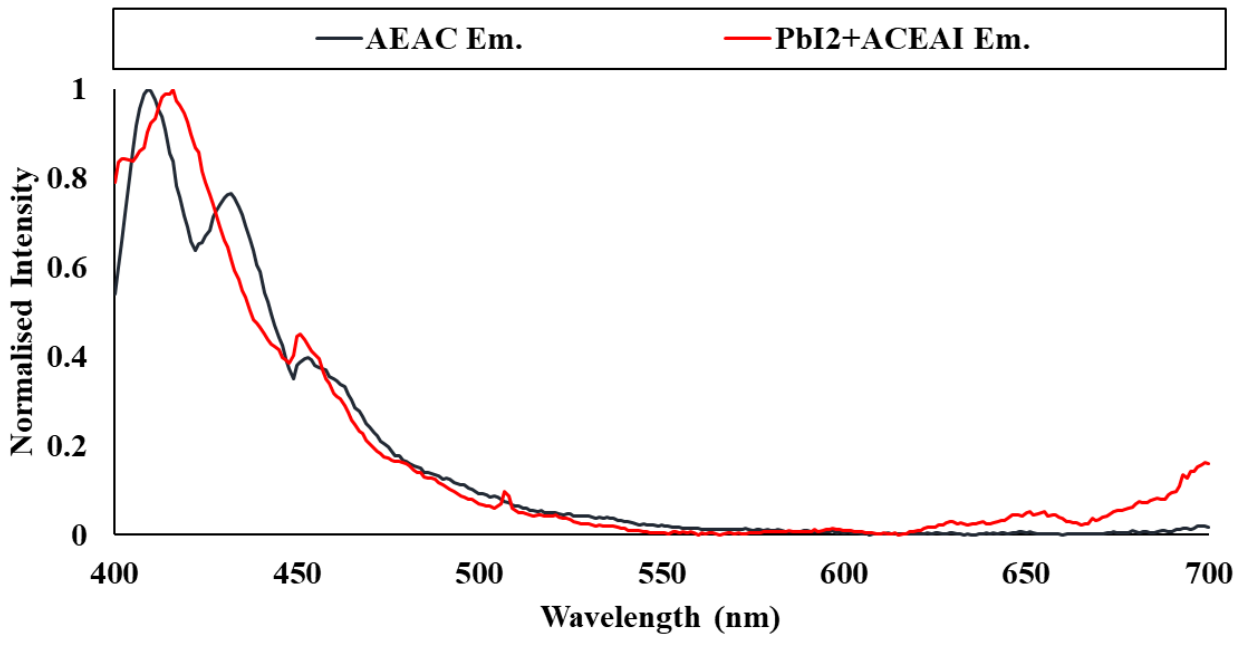

(B)

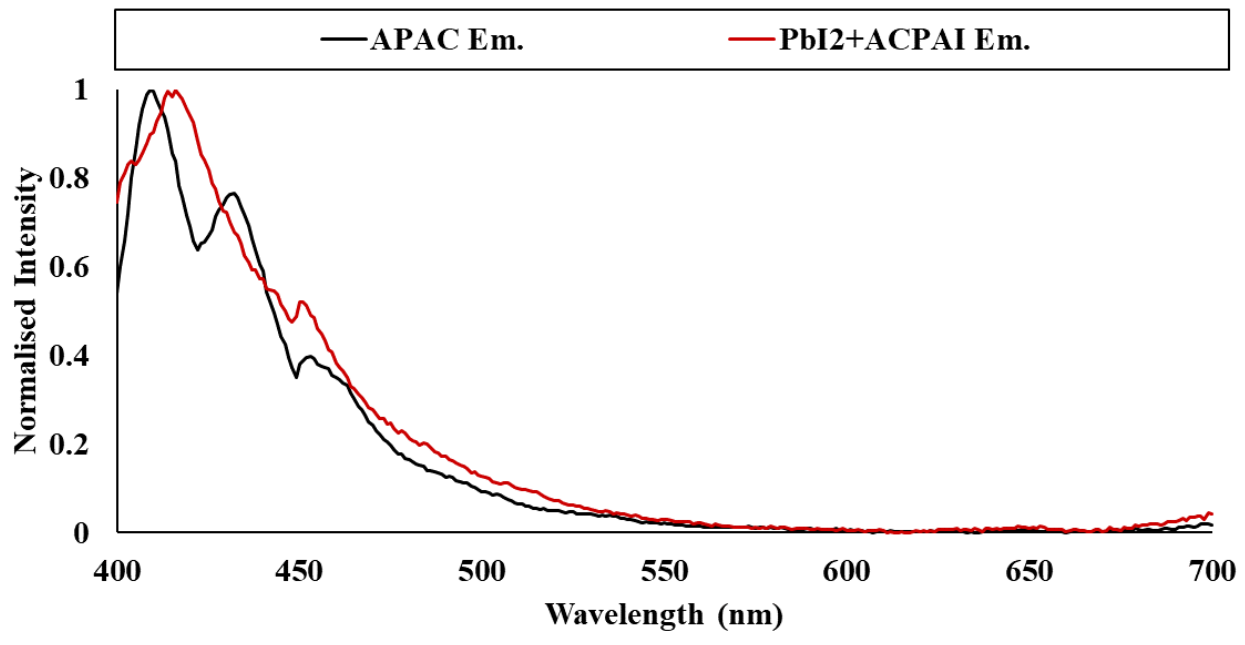

(C)

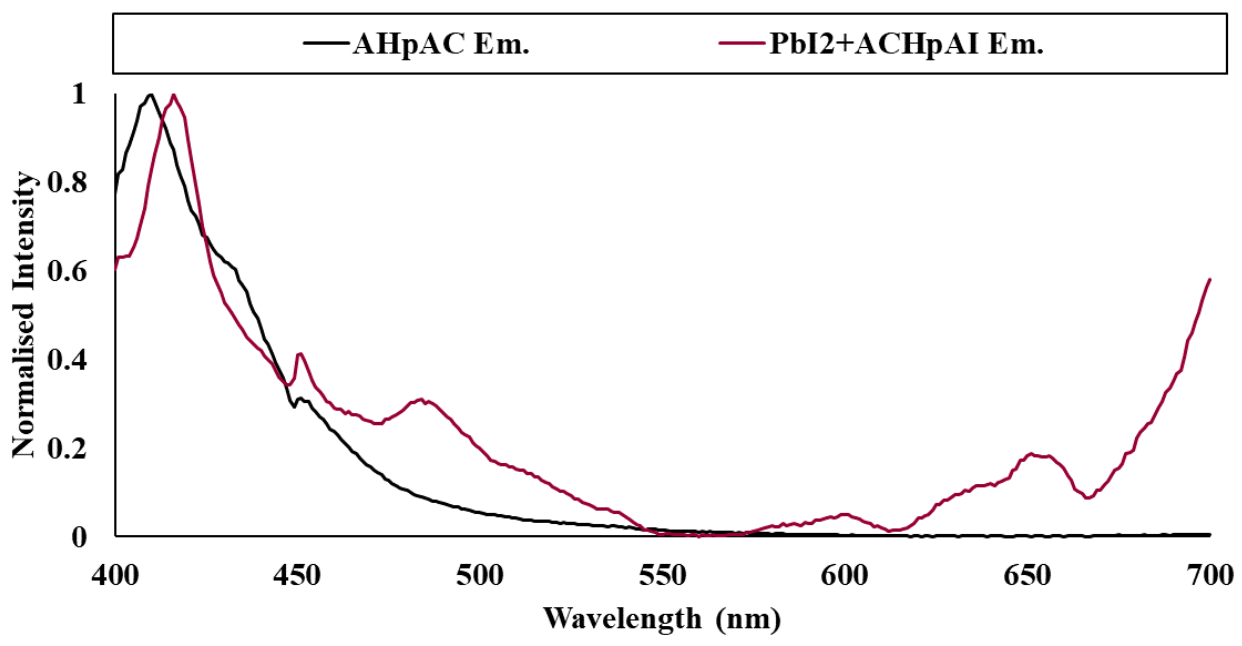

Figure 10u. (A) Comparison between the emission spectra of AEAC, and $\mathrm{PbI}_{2}+\mathrm{ACEAI}$. (B) Comparison between the emission spectra of APAC, and PbI $\mathrm{P}_{2}+\mathrm{ACPAI}$. (C) Comparison between the emission spectra of $A H p A C$, and $\mathrm{PbI}_{2}+A C H p A I$. 
The clear dominance in emission by anthracene and lack of any perovskite like emission suggests that the intercalation reaction failed to produce a high quality perovskite phase. This result indicates that either no perovskite phase is present, or that a low quality perovskite phase is present that possess too many crystallographic defects to be highly fluorescent. While it may be possible for a two dimensional lead chloride perovskite to absorb and transfer energy to anthracene, lead bromide and iodide two-dimensional perovskites would not possess a band gap energy that is large enough to induce anthracene emission. Therefore, based upon absorption and emission data, the intercalation samples are likely to be a heterogenous mixture of partially aggregated and or degraded lead halide nanocrystals and ACAAX salts. 


\section{Electronic Excitation of (Anthracene-9-Carboxamido)Alkylaminium Halide Salts with Lead}

\section{Halide Nanocrystals}

The nine samples demonstrate great variability in their electronic excitation spectra (see Figures 10v, $10 w, 10 x)$. Overlaying the excitation spectra with their respective absorption spectra revels a negative correlation between the two for all samples except $\mathrm{PbBr}_{2}+\mathrm{APACBr}$. Such a correlation suggests that the concentration of anthracene functional groups in eight of the samples is high enough to result in self-quenching of sample fluorescence. In other words, the peak absorption wavelengths of anthracene are effectively absorbed before being able to excite the bulk of the sample. Therefore, the wavelengths of light that are not principally absorbed will be able to penetrate and excite the majority of the sample and therefore induce the greatest amount of emission.

Comparing the electronic excitation spectra of the intercalated samples with that of the pure AAAC solutions shows that some samples demonstrate positive correlations between peak excitation wavelengths and others negative correlation. For $\mathrm{PbCl}_{2}+\mathrm{ACEACl}$, the sample displays a positive correlation between the peaks of its excitation spectrum and that of pure AEAC. However, it must be remembered that there is a negative correlation between the absorption spectra of the sample and their excitation spectra. Therefore, while some samples display positive correlations in their electronic excition spectra with those of the pure AAAC solutions, this is only an apparent positive correlation because the peaks in excitation are due to non-peak absorption. If the concentration of the samples were lowered, then the excitation peaks would likely shift, changing the correlation with the excitation spectra of the AAACs. A similar thinking must be applied to apparent negative correlations.

Therefore, the negative correlation between the samples absorbance and excitation spectra, and the clear redshift in absorption peaks when compared to that of pure AEAC, overall suggests that an interaction has taken place between the organic and inorganic reagents. This conclusion is more apparent in the chloride sample $\mathrm{PbCl}_{2}+\mathrm{ACEACl}$ which displays a unique excitation peak at $312 \mathrm{~nm}$. The lack of an equivalent excitation peak in the pure anthracene solution suggests that this feature is indicative of a unique electronic transition that can occur in this sample. One possibility is that this is the result of a two dimensional perovskite phase. The peak excitation wavelength of 2D RuddlesdenPopper chloride perovskite films tends to be $\sim 332 \mathrm{~nm}$. While the excitation feature of $\mathrm{PbCl}_{2}+$ $\mathrm{ACEACl}$ is at a higher wavelength of $312 \mathrm{~nm}$, if the small, dense particles that are present within the sample are even partially perovskite, the small size of the particles would induce further quantum confinement and therefore possess an even larger band gap than usual. Neither bromide nor iodide samples display similar peaks, though such a peak may be obscured and not detected due being too low in energy to induce the peak emission of the sample. 
For the samples containing APAC, both the bromide and iodide samples display a negative correlation with the excitation spectrum of pure APAC. The positive correlation between the excitation and absorption spectra of $\mathrm{PbBr}_{2}+\mathrm{ACPABr}$ means that a similar correlation with the excitation spectra of pure APAC is not just an apparent positive correlation. Therefore, this correlation suggests that there has been little interaction between $\mathrm{ACPABr}$ and $\mathrm{PbBr}_{2} \mathrm{NCs}$. The iodide on the other hand does display a negative correlation between excitation and absorption and therefore the positive correlation with APAC excitation is only apparent, suggesting that a more meaningful interaction between the two reagents has taken place.

All three samples possessing AHpAC display a positive apparent correlation with the excitation spectrum of $\mathrm{AHpAC}$, therefore indicating some kind of significant interaction between the two reagents and a resulting mixed phase. 
(A)

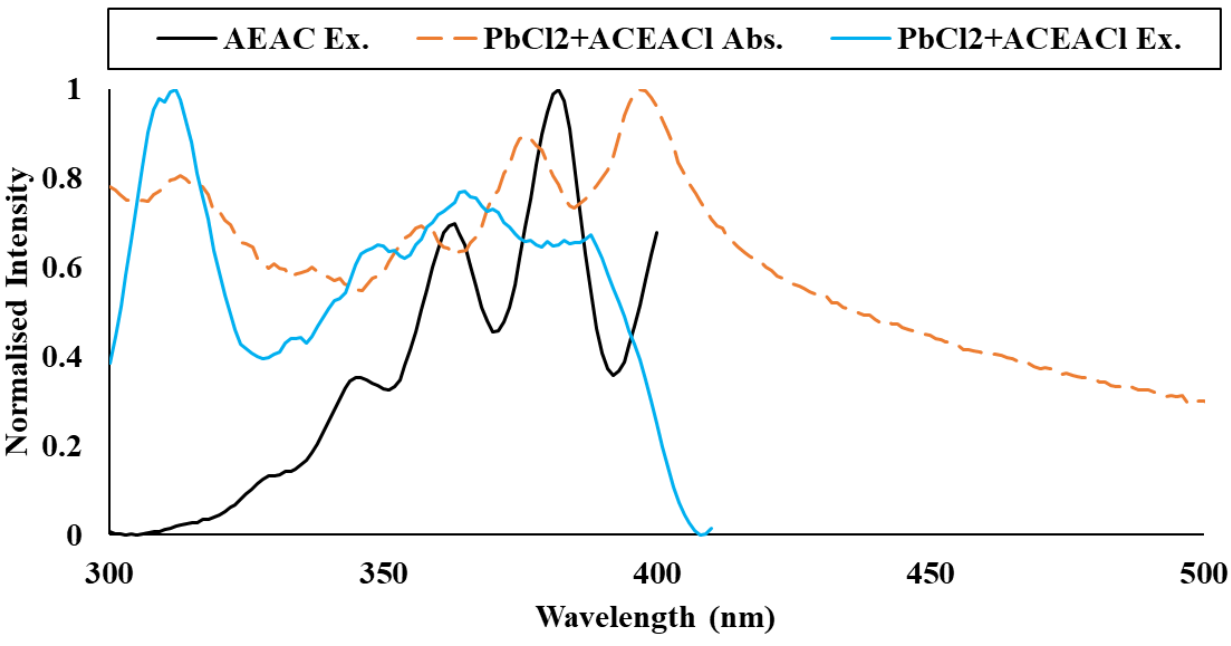

(B)

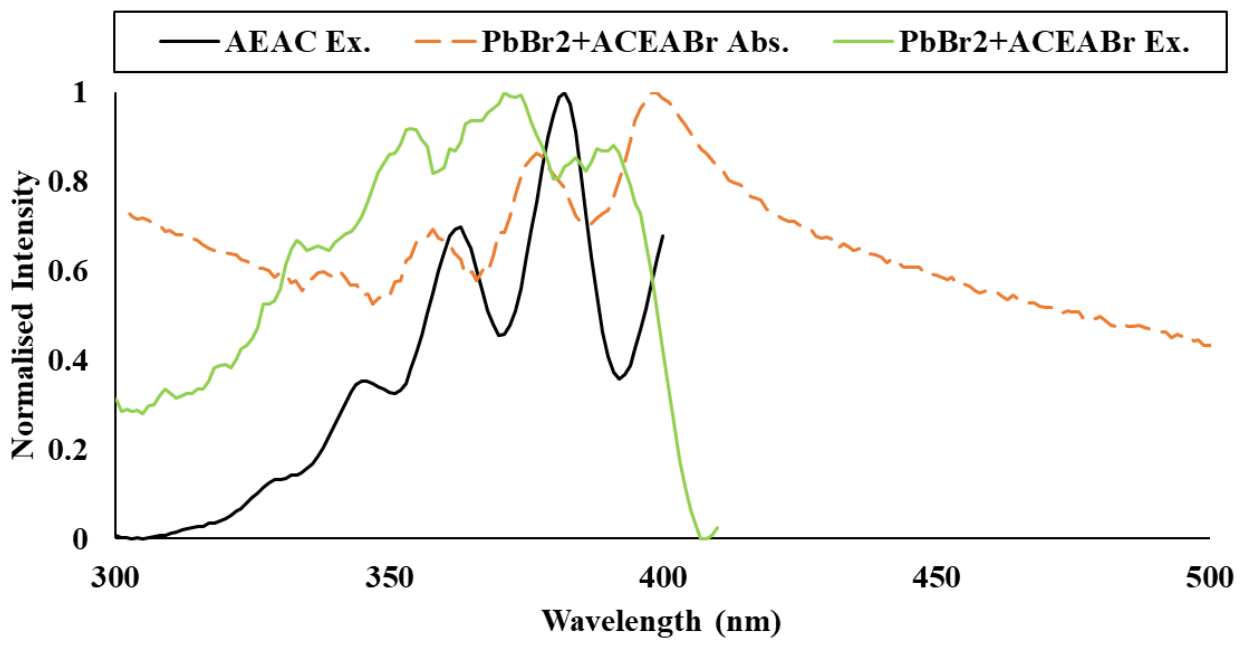

(C)

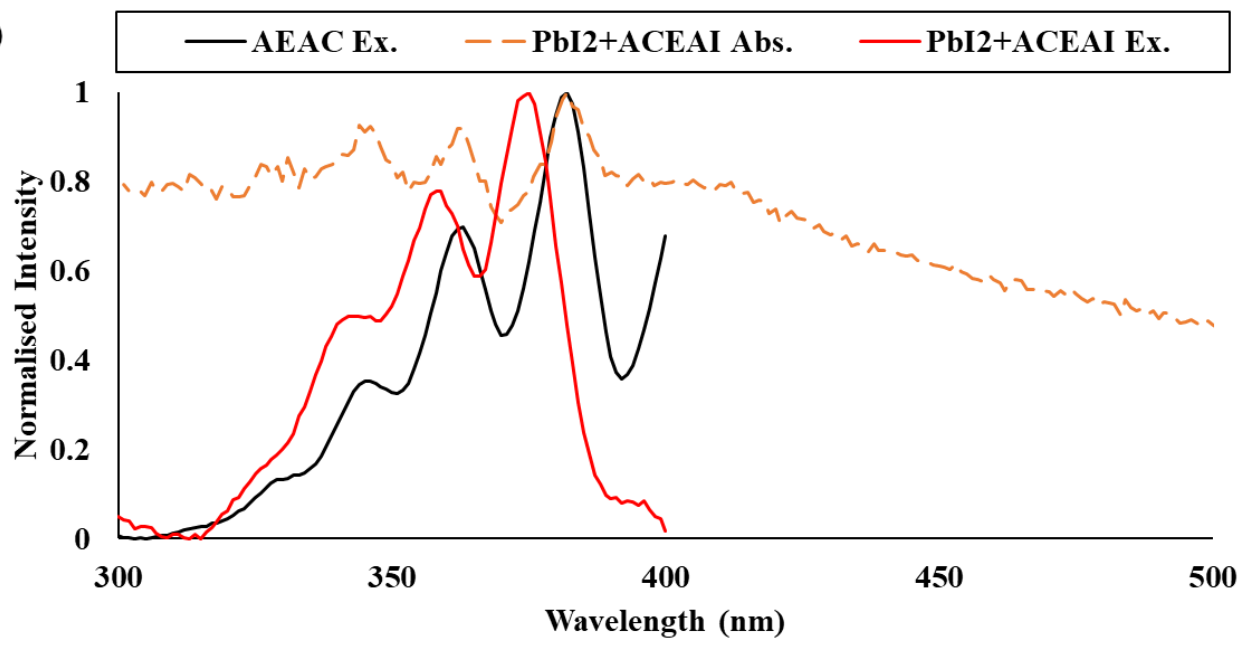

Figure 10v. (A) Comparison between the absorption and excitation spectra of $\mathrm{PbCl}_{2}+\mathrm{ACEACl}$ and the excitation of AEAC. (B) Comparison between the absorption and excitation spectra of $\mathrm{PbBr}_{2}+\mathrm{ACEABr}$ and the excitation of AEAC. (C) Comparison between the absorption and excitation spectra of $\mathrm{PbI}_{2}+\mathrm{ACEAI}$ and the excitation of AEAC. 
(A)

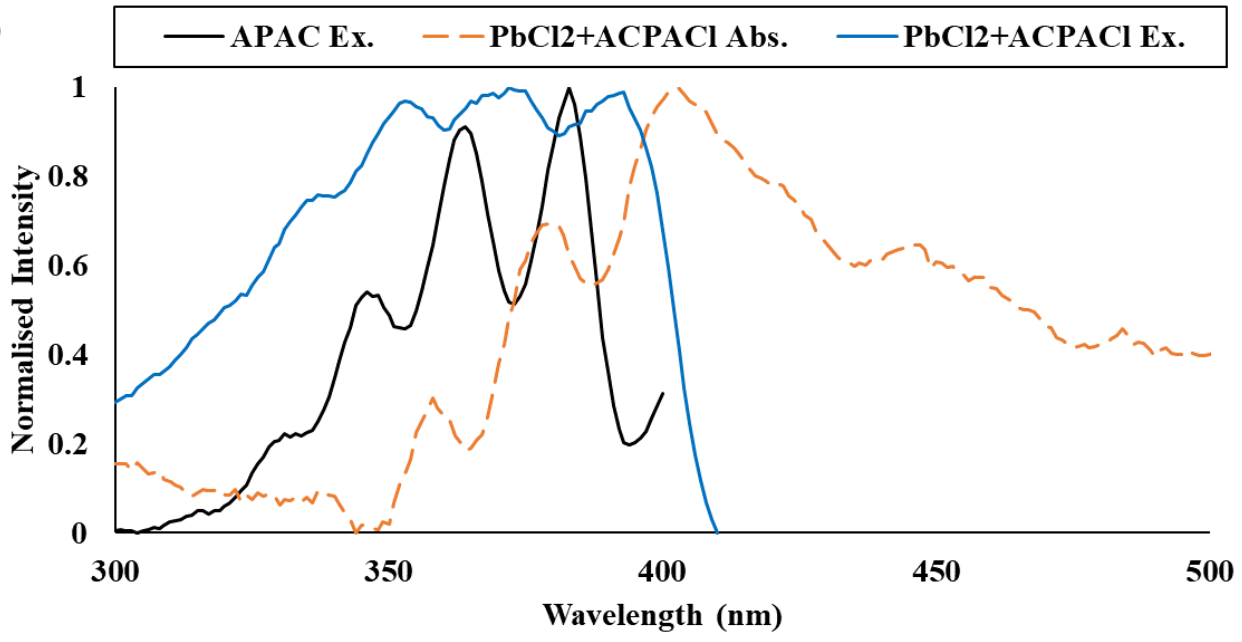

(B)

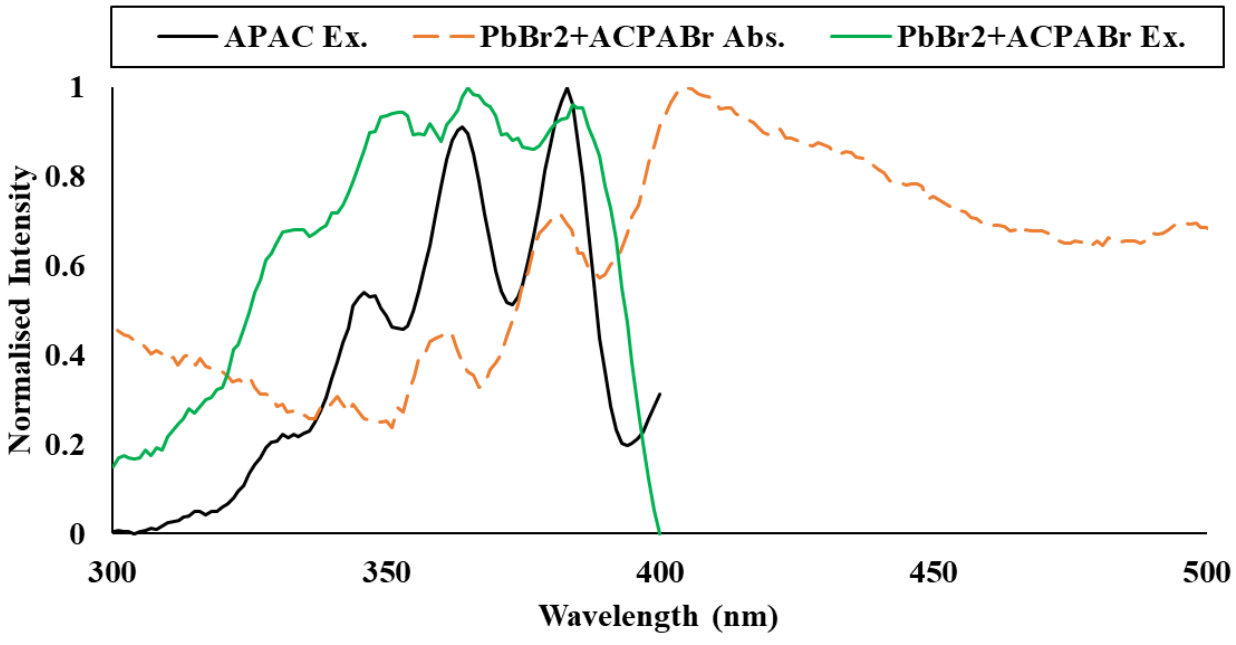

(C)

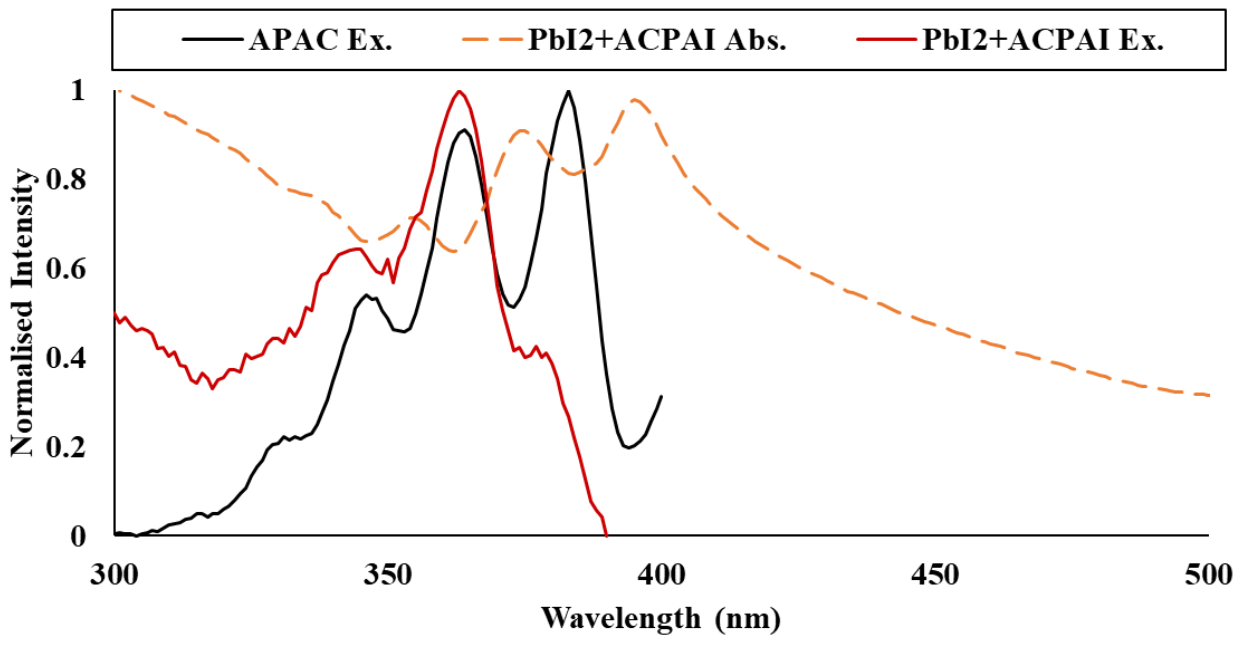

Figure 10w. (A) Comparison between the absorption and excitation spectra of $\mathrm{PbCl}_{2}+\mathrm{ACPACl}$ and the excitation of APAC. (B) Comparison between the absorption and excitation spectra of $\mathrm{PbBr}_{2}+\mathrm{ACPABr}$ and the excitation of APAC. (C) Comparison between the absorption and excitation spectra of $\mathrm{PbI}_{2}+\mathrm{ACPAI}$ and the excitation of APAC. 
(A)

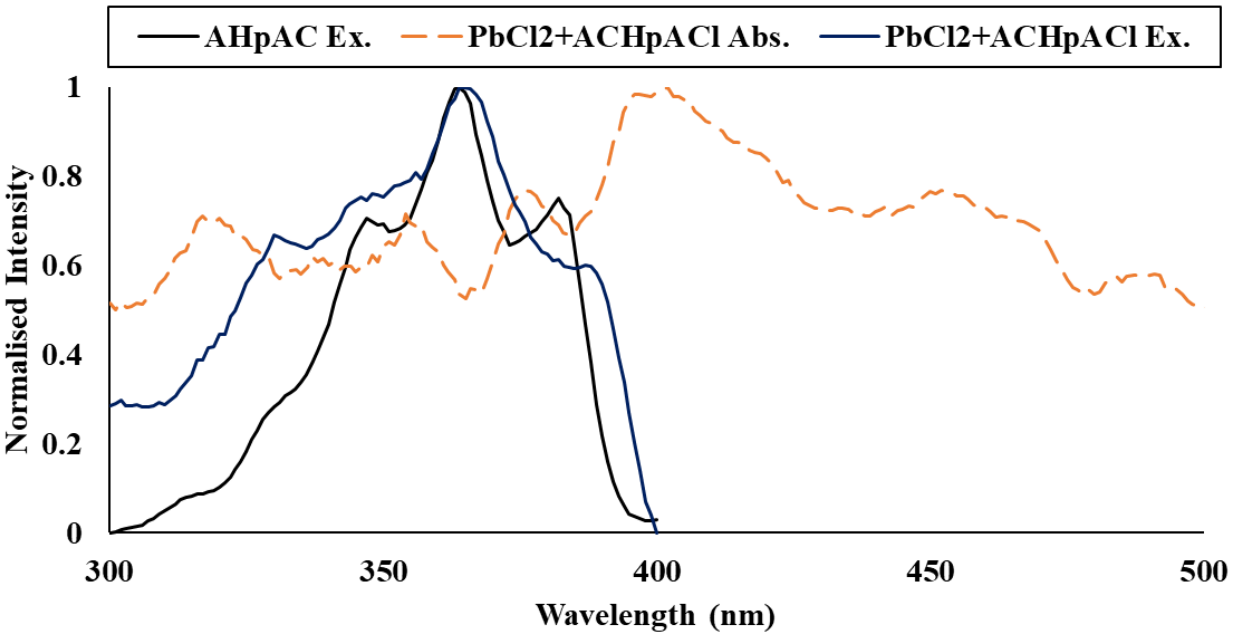

(B)

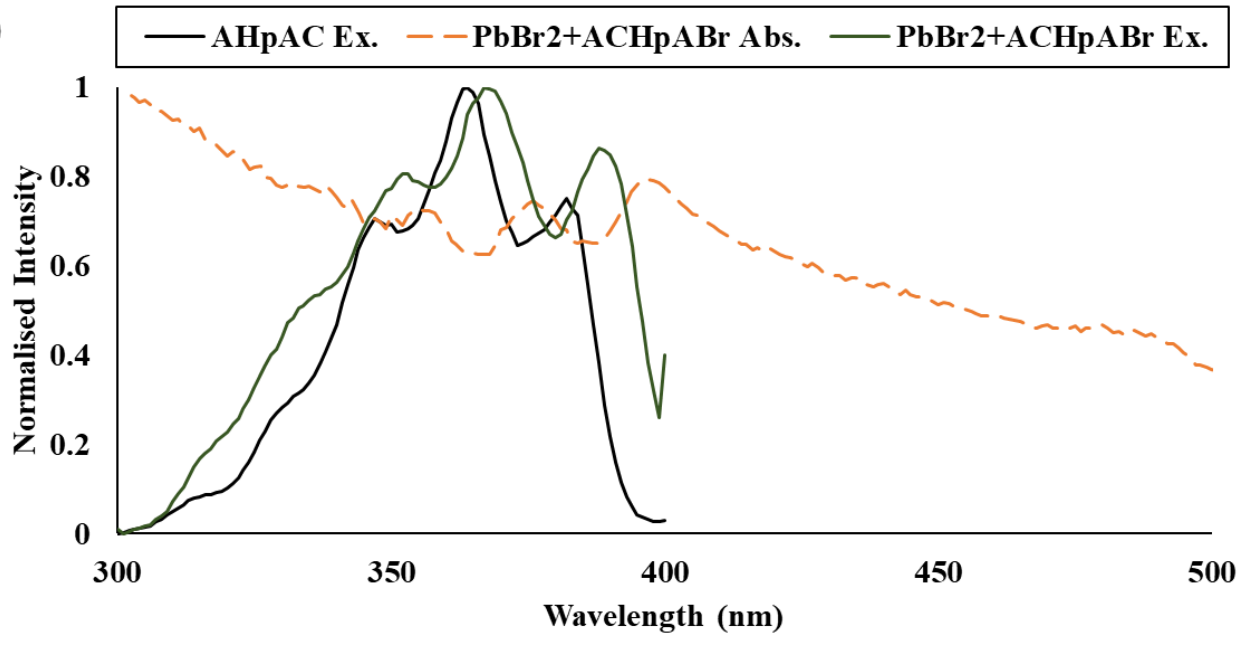

(C)

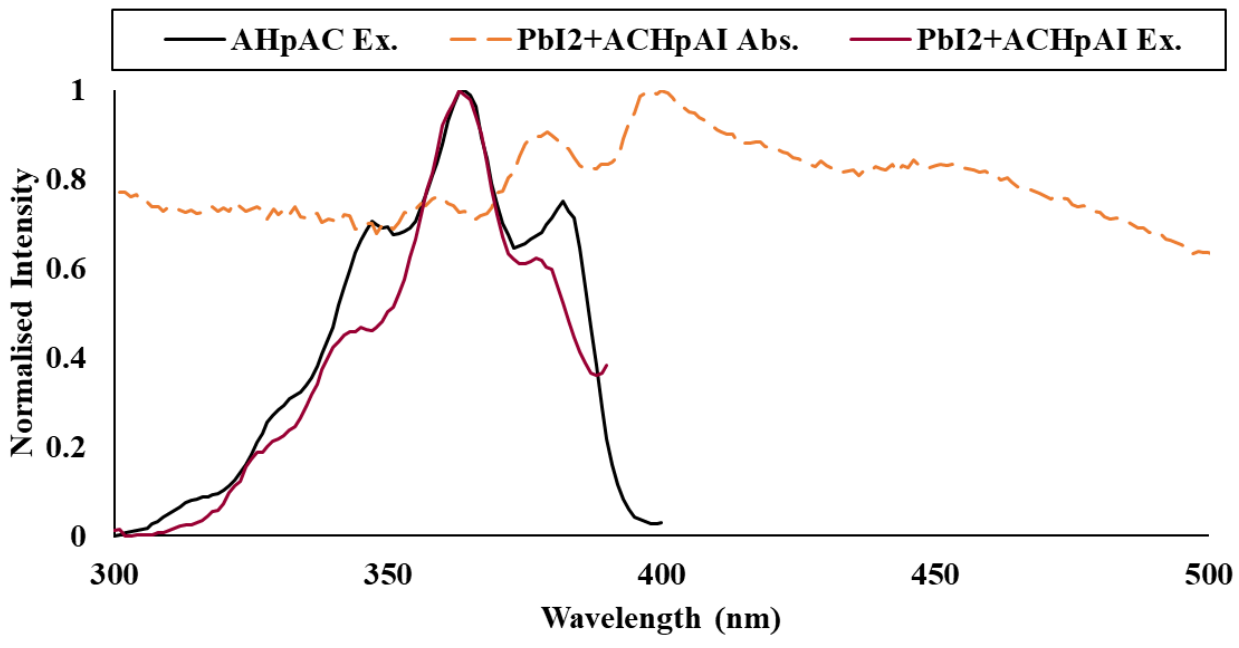

Figure 10x. (A) Comparison between the absorption and excitation spectra of $\mathrm{PbCl}_{2}+\mathrm{ACHpACl}$ and the excitation of AHpAC. (B) Comparison between the absorption and excitation spectra of $\mathrm{PbBr}_{2}+\mathrm{ACHpABr}$ and the excitation of AHpAC. (C) Comparison between the absorption and excitation spectra of $\mathrm{PbI}_{2}+\mathrm{ACHpAI}$ and the excitation of AHpAC. 
Photoluminescence Lifetime of (Anthracene-9-Carboxamido)Alkylaminium Halide Salts with Lead Halide Nanocrystals

Mixed phases of 2-(anthracene-9-carboxamido)ethan-1-aminium halides (ACEAX) with any lead halide results in a significant increase in the emission decay rate (see Figure 10y). The significant reduction in emission lifetime suggests that there is an energy transfer process occurring that is competing with the process of emission. While excimer formation is a common cause of lifetime reduction, there is no excimer emission from any of these samples. Therefore, there must be either a new phase that is capable of accepting energy from the anthracene functional groups, or new nonradiative decay states present within the mixed phase. Whether or not a perovskite phase is present

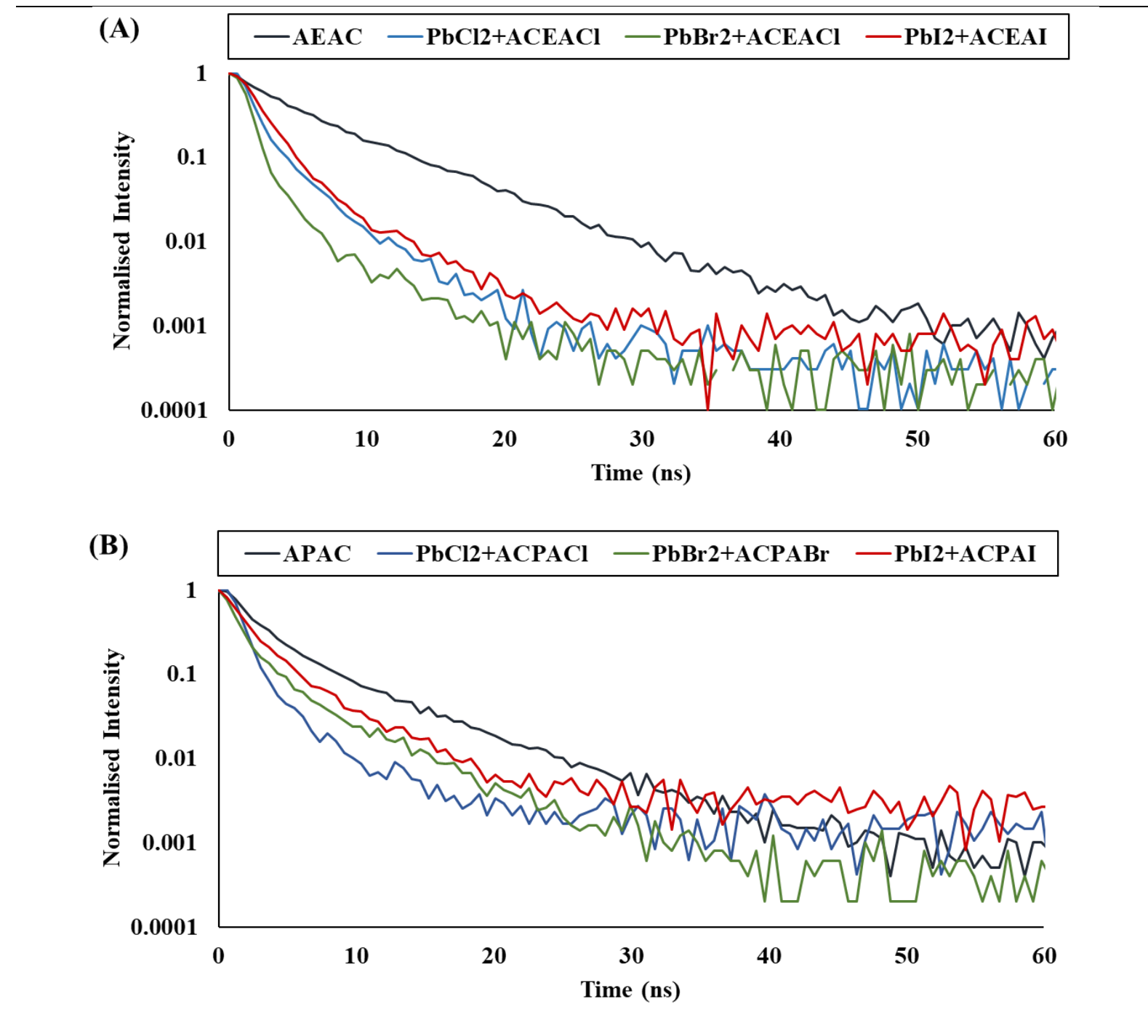

Figure 10y. (A) Comparison between the emission decay of pure AEAC and mixed phases of $P b X_{2}$ and $A C E A X(X=C l, B r$, and $X)$. (B) Comparison between the emission decay of pure $A P A C$ and mixed phases of $P b X_{2}$ and $A C P A X(X=C l, B r$, and $I)$. 
is difficult to determine due to the lack of emission or excitation features. The lack of an additional emission feature despite the clear reduction in emission lifetime of anthracene suggests that the material that is siphoning the energy is poorly emissive in the UV-Visible spectral range. One possibility is that if there is a perovskite phase, it may possess significant crystallographic defects. These defects could act as trap sites, facilitating non-radiative recombination and quenching the overall fluorescence of the sample.

One sample that may have possessed a perovskite phase was the sample $\mathrm{PbCl}_{2}+\mathrm{ACEACl}$. This sample displayed a unique excitation peak at $312 \mathrm{~nm}$ which is comparable to the excitation peaks of two dimensional lead chloride perovskites. If this was a perovskite peak, then it would mean that the inorganic lead chloride semiconductor is absorbing and transferring energy to the anthracene functional groups. To test this, the lifetime of anthracene emission when excited with $312 \mathrm{~nm}$ light would need to be compared between that of pure AEAC and $\mathrm{PbCl}_{2}+\mathrm{ACEACl}$. However, the laser used to perform these measurements has a wavelength of $374 \mathrm{~nm}$, lower in energy than the $312 \mathrm{~nm}$ light that resonates with this excitation feature. Therefore, it could not be tested whether or not this feature boosted anthracene emission.

All three samples display a comparable emission lifetime, suggesting that the halogen that is present within the sample does not have a large influence on the rate of decay.

Mixed phases of ACPAX with any lead halide display comparable emission lifetimes to those of the AEAC samples. Therefore, while in a mixed phase, the length of the alkyl chain in an AAAC molecule has little influence on the emission decay rate.

\section{Photoluminescence Quantum Yield of (Anthracene-9-Carboxamido)Alkylaminium Halide Salts with Lead Halide Nanocrystals}

All samples demonstrated low photoluminescence quantum yield (PLQE) values when illuminated with $375 \mathrm{~nm}$ light.

All iodide samples displayed PLQE values of $0 \%$, likely a result of the significant absorption of light by non-fluorescent lead iodide.

For the chloride series, $\mathrm{PbCl}_{2}+\mathrm{ACEACl}, \mathrm{PbCl}_{2}+\mathrm{ACPACl}$, and $\mathrm{PbCl}_{2}+\mathrm{ACHpACl}$, the respective PLQE values were $1.57 \%, 3.10 \%$, and $0.75 \%$. Additionally, targeting the high energy excitation region of $\mathrm{PbCl}_{2}+\mathrm{ACEACl}$ with $310 \mathrm{~nm}$ light results in a comparable efficiency value of $1.82 \%$

For the bromides, $\mathrm{PbBr}_{2}$ with $\mathrm{ACEABr}, \mathrm{PbBr}_{2}$ with $\mathrm{ACPABr}$, and $\mathrm{PbBr}_{2}$ with $\mathrm{ACHpABr}$, the respective PLQE values were significantly higher at 1.99\%, 5.66\%, and 6.53\%. 


\section{0e. Concluding Remarks on the Attempts to Intercalate (Anthracene-9-carboxamido) Halide Salts into Lead Halide Nanocrystals}

Attempts to intercalation ACAAX salts into lead halide nanocrystals results in the precipitation of a mixed phase between the two reagents. Based upon the transmission electron microscope images and energy-dispersive X-ray spectroscopy, the majority of the samples are composed of a mixed phase of lead halide and ACAAX salt. For the chlorides, two of the samples demonstrate well preserved lead chloride nanocrystals $\left(\mathrm{PbCl}_{2} \mathrm{NCs}\right)$ within the organic material. For the bromides, any lead that is present is in the form of shards of coalesced nanocrystals that are segregated from the organic material. The iodides appear to be composed of a homogenous phase of mixed ACAAI salt and $\mathrm{PbI}_{2}$. The degradation of the nanoparticles is likely a result of an interaction between the stabilising ligands and the ACAAX salt.

All samples demonstrate monomer anthracene emission; however, they also display reduced emission lifetimes. The reduced emission lifetime indicates that there is a competitive process that is quenching anthracene emission. One possibility is that within the mixed phase there is a nanomaterial to which energy is being transferred to. If this material is a perovskite, then it is poorly fluorescent. Poor fluorescence may indicate the presence of significant crystallographic defects, allowing for high rates of non-radiative recombination. One way to prove or disprove the existence of a perovskite phase would be to perform an X-Ray diffraction analysis of the sample. Unfortunately, no X-Ray diffractometer was available for use during the course of this research. Future work could focus on collecting such data on these samples.

Based on these results, it can be concluded that the synthesis of 2D Ruddlesden-Popper perovskite nanocrystals cannot be done via the method of intercalation. The ACAAX salts facilitate the degradation of the precursor lead halide nanocrystals resulting in a mixed phase between the two reagents. However, there is an interaction taking place between the ACAAX salts and as the emission lifetime of the salts is reduced drastically, indicating a competitive process is taking place that is quenching the fluorescence of anthracene 


\section{Coordination of Anthracene Based Ligands to Open-Air Synthesised $\mathrm{CsPbX}_{3}$ Nanocrystals}

\section{1a. Open-Air Synthesis of $\mathrm{CsPbX}_{3}$ and $\mathrm{CsPbX}_{3}-\mathrm{AL}$ Nanocrystals}

A simple open-air method for the synthesis of caesium lead trihalides $\left(\mathrm{CsPbX}_{3}\right)$ was experimented with in attempts to incorporate anthracene based ligands (ALs) onto the surface of the nanocrystals.

The unaltered method is as follows. Refer to Table 11a for reagent details.

\begin{tabular}{cccccc}
\hline Compound & $\begin{array}{c}\text { Molar } \\
\text { Equivalents }\end{array}$ & mmol & Mass/Volume & $\begin{array}{c}\text { Density } \\
\left(\mathbf{g ~ m L}^{-\mathbf{1}}\right)\end{array}$ & $\begin{array}{c}\text { Molar Mass } \\
\left(\mathbf{g ~ m o l}^{-\mathbf{1}}\right)\end{array}$ \\
$\mathbf{C s}_{\mathbf{2}} \mathbf{C O}_{\mathbf{3}}$ & 1 & 0.1 & $32.6 \mathrm{mg}$ & - & 325.82 \\
$\mathbf{C s B r}$ & 1 & 0.1 & $21.3 \mathrm{mg}$ & - & 212.81 \\
$\mathbf{P b B r}_{2}$ & 1 & 0.1 & $36.7 \mathrm{mg}$ & - & 367.01 \\
$\mathbf{9 0 \%}$ Oleic Acid & 16 & 1.6 & $0.5 \mathrm{~mL}$ & 0.895 & 282.47 \\
$\mathbf{8 0 - 9 0 \%}$ Oleylamine & 1 & 0.1 & $0.035 \mathrm{~mL}$ & 0.813 & 267.49 \\
Anhydrous DMF & 323 & 161.5 & $2.5 \mathrm{~mL}$ & 0.944 & 73.09 \\
Anhydrous Toluene & 2352 & 235.2 & $25 \mathrm{~mL}$ & 0.867 & 92.14 \\
& & & & & \\
\hline
\end{tabular}

Table 11a. Table of Reagents for the atmospheric synthesis of $\mathrm{Cs}_{\mathrm{PbBr}}$.

\section{Step 1: Preparation of Caesium Source}

$0.1 \mathrm{mmol}$ of $\mathrm{Cs}_{2} \mathrm{CO}_{3}, 0.1 \mathrm{mmol}$ of $\mathrm{CsX}$, and $25 \mathrm{~mL}$ of anhydrous toluene was added to a $50 \mathrm{~mL}$ beaker covered with a petri dish to prevent the loss of toluene vapour. The mixture was stirred at 110 ${ }^{\circ} \mathrm{C}$ for at least one hour, after which the solution was allowed to cool to room temperature by removing the heating source.

\section{Step 2: Preparation of lead halide source}

$0.1 \mathrm{mmol}$ of $\mathrm{PbX}_{2}, 1.6 \mathrm{mmol}$ of oleic acid (OA), $0.1 \mathrm{mmol}$ of oleylamine (OAm), and $2.5 \mathrm{~mL}$ of dimethylformamide will be combined in a small vial. The mixture will be stirred at $110{ }^{\circ} \mathrm{C}$ for one hour. After an hour, the heating is adjusted to allow the solution to cool and maintain a temperature of $80{ }^{\circ} \mathrm{C}$ 


\section{Step 3: Synthesis Caesium Lead Halide Nanocrystals}

The $80{ }^{\circ} \mathrm{C}$ lead halide precursor solution was rapidly added to a vigorously stirred caesium source. 10 seconds after the addition the reaction was quenched by cooling in a water-ice bath.

\section{Step 4: Isolating the Nanocrystals}

$10 \mathrm{~mL}$ samples of the reaction mixture were placed into centrifuge tubes, after which between 10 to $30 \mathrm{~mL}$ of anhydrous $\mathrm{n}$-butanol was added to induce precipitation of the nanocrystals. The particles were collected by condensing them at the bottom of the vessel by centrifuging these mixtures at 5000 rpm for 5 mins. The supernatant was then discarded and the solid resuspended in $5 \mathrm{~mL}$ of toluene.

\section{Step 4: Washing Procedure}

5 to $15 \mathrm{~mL}$ of anhydrous $n$-butanol was added to the toluene nanocrystal solution to again induce precipitation. Centrifugation at $5000 \mathrm{rpm}$ was used to condense this precipitate, after which the supernatant is removed. This process was repeated multiple times after which the final product was resuspended in $5 \mathrm{~mL}$ of toluene.. This final solution was centrifuged between 1500 and $5000 \mathrm{rpm}$ for 2 mins without $n$-butanol to isolate nanoparticles of different sizes. The original solution was kept and each solid was resuspended in anhydrous toluene and kept as separate samples.

\section{$\underline{\text { Modifications }}$}

The original synthesis method was altered to incorporate the ALs. Initial attempts observed the consequences of replacing one or both of the fatty ligands with anthracene based alternatives. The first of these involved replacing oleic acid with anthracene-9-carboxylic acid (AC), see Table $\mathbf{1 1 b}$ for details.

\begin{tabular}{|c|c|c|c|c|c|}
\hline Compound & Equivalents & mmol & Mass/Volume & $\begin{array}{l}\text { Density } \\
\left(g_{\text { mL }}^{-1}\right)\end{array}$ & $\begin{array}{l}\text { Molar Mass } \\
\qquad\left(\mathrm{g} \mathrm{mol}^{-1}\right)\end{array}$ \\
\hline $\mathbf{A C}$ & 16 & 1.6 & $355.6 \mathrm{mg}$ & - & 222.24 \\
\hline $80-90 \%$ Oleylamine & 1 & 0.1 & $0.035 \mathrm{~mL}$ & 0.813 & 267.49 \\
\hline \multicolumn{6}{|c|}{$\begin{array}{l}\text { Table 11b. Table of ligands for the modified open-air synthesis of } \mathrm{Cs}_{\mathrm{PbBr}} \text {, replacing oleic acid } \\
(\mathrm{OA}) \text { with anthracene-9-carboxylic acid }(A C) \text {. }\end{array}$} \\
\hline
\end{tabular}

The second experiment substituted oleylamine (OAm) with one of the three $N$ (aminoalkyl)anthracene-9-carboxamides (AAAC). See Table 11c for details. 


\begin{tabular}{|c|c|c|c|c|c|}
\hline Compound & $\begin{array}{c}\text { Molar } \\
\text { Equivalents }\end{array}$ & mmol & Mass/Volume & $\begin{array}{l}\text { Density } \\
\left(\mathrm{g} \mathrm{mL}^{-1}\right)\end{array}$ & $\begin{array}{c}\text { Molar Mass } \\
\quad\left(\mathrm{g} \mathrm{mol}^{-1}\right)\end{array}$ \\
\hline $90 \%$ Oleic Acid & 16 & 1.6 & $0.5 \mathrm{~mL}$ & 0.895 & 282.47 \\
\hline AEAC & 1 & 0.1 & $26.4 \mathrm{mg}$ & - & 264.33 \\
\hline or APAC & 1 & 0.1 & $278.3 \mathrm{mg}$ & - & 278.35 \\
\hline or AHpAC & 1 & 0.1 & $33.4 \mathrm{mg}$ & - & 334.46 \\
\hline
\end{tabular}

The third experiment replaced both OA and OAm with AC and an AAAC. See Table 11d for details.

\begin{tabular}{|c|c|c|c|c|c|}
\hline Compound & $\begin{array}{c}\text { Molar } \\
\text { Equivalents }\end{array}$ & mmol & Mass/Volume & $\begin{array}{l}\text { Density } \\
\left(\mathrm{g} \mathrm{mL}^{-1}\right)\end{array}$ & $\begin{array}{c}\text { Molar Mass } \\
\quad\left(\mathrm{g} \mathrm{mol}^{-1}\right)\end{array}$ \\
\hline $\mathrm{AC}$ & 16 & 1.6 & $355.6 \mathrm{mg}$ & - & 222.24 \\
\hline AEAC & 1 & 0.1 & $26.4 \mathrm{mg}$ & & 264.33 \\
\hline APAC & 1 & 0.1 & $278.3 \mathrm{mg}$ & - & 278.35 \\
\hline AHpAC & 1 & 0.1 & $33.4 \mathrm{mg}$ & - & 334.46 \\
\hline \multicolumn{6}{|c|}{$\begin{array}{l}\text { Table 11d. Table of ligands for the modified open-air synthesis of } \mathrm{Cs}_{\mathrm{PBBr}}, \text { replacing oleic acid } \\
\text { and oleylamine with anthracene-9-carboxylic acid }(A C) \mathrm{N} \text {-(aminoalkyl)anthracene-9- } \\
\text { carboxamide }(A A A C) \text {. }\end{array}$} \\
\hline
\end{tabular}

Alongside these experiments, the anthracene ligands were added to the standard reaction mixtures containing the fatty acid ligands, see Table 11e and Table $11 f$.

\begin{tabular}{|c|c|c|c|c|c|}
\hline Compound & $\begin{array}{c}\text { Molar } \\
\text { Equivalents }\end{array}$ & mmol & Mass/Volume & $\begin{array}{l}\text { Density } \\
\left(\mathrm{g} \mathrm{mL}^{-1}\right)\end{array}$ & $\begin{array}{c}\text { Molar Mass } \\
\left(\mathrm{g} \mathrm{mol}^{-1}\right)\end{array}$ \\
\hline $\mathbf{A C}$ & 16 & 1.6 & $355.6 \mathrm{mg}$ & - & 222.24 \\
\hline 90\% Oleic Acid & 16 & 1.6 & $0.5 \mathrm{~mL}$ & 0.895 & 282.47 \\
\hline $80-90 \%$ Oleylamine & 1 & 0.1 & $0.035 \mathrm{~mL}$ & 0.813 & 267.49 \\
\hline
\end{tabular}




\begin{tabular}{cccccc}
\hline Compound & $\begin{array}{c}\text { Molar } \\
\text { Equivalents }\end{array}$ & mmol & Mass/Volume & $\begin{array}{c}\text { Density } \\
\left(\mathbf{g ~ m L}^{-1}\right)\end{array}$ & $\begin{array}{c}\text { Molar Mass } \\
\left(\mathbf{g ~ m o l}^{-1}\right)\end{array}$ \\
90\% Oleic Acid & 16 & 1.6 & $0.5 \mathrm{~mL}$ & 0.895 & 282.47 \\
$\mathbf{8 0 - 9 0 \% ~ O l e y l a m i n e ~}$ & 1 & 0.1 & $0.035 \mathrm{~mL}$ & 0.813 & 267.49 \\
AEAC & 1 & 0.1 & $26.4 \mathrm{mg}$ & - & 264.33 \\
or APAC & 1 & 0.1 & $278.3 \mathrm{mg}$ & - & 278.35 \\
or AHpAC & 1 & 0.1 & $33.4 \mathrm{mg}$ & - & 334.46 \\
& & & & & \\
\hline
\end{tabular}

Table 11f. Table of ligands for the modified atmospheric synthesis of $\mathrm{CsPbBr}$, , replacing oleylamine with a $\mathrm{N}$-(aminoalkyl)anthracene-9-carboxamide (AAAC).

Attempts to synthesis the caesium lead trichloride $\left(\mathrm{CsPbCl}_{3}\right)$ and caesium lead triiodide $\left(\mathrm{CsPbI}_{3}\right)$ perovskites were also carried out (see Table 11g).

\begin{tabular}{|c|c|c|c|c|c|}
\hline \multirow[t]{3}{*}{ Compound } & Molar & mmol & Mass/Volume & Density & Molar Mass \\
\hline & \multicolumn{5}{|c|}{ Equivalents } \\
\hline & & & & $\left(\mathrm{g} \mathrm{mL}^{-1}\right)$ & $\left(\mathrm{g} \mathrm{mol}^{-1}\right)$ \\
\hline $\mathbf{P b C l}_{2}$ & 1 & 0.1 & $27.81 \mathrm{mg}$ & - & 278.10 \\
\hline $\mathrm{CsCl}$ & 1 & 0.1 & $16.84 \mathrm{mg}$ & - & 168.36 \\
\hline $\mathbf{P b I}_{2}$ & 1 & 0.1 & $46.10 \mathrm{mg}$ & - & 461.01 \\
\hline CsI & 1 & 0.1 & $25.98 \mathrm{mg}$ & - & 259.81 \\
\hline \multicolumn{6}{|c|}{ Table 11g. Table of ligands for the modified atmospheric synthesis of $\mathrm{Cs} \mathrm{PbBr}_{3}$, replacing } \\
\hline & (amin & & heramida 140 & & \\
\hline
\end{tabular}

\section{$\underline{\text { Analysis }}$}

All samples had their absorption spectrum measured on a Varian Cary 50 Scan UV/Vis Spectrophotometer, their photoluminescence properties including emission, excitation, photoluminescence quantum yield, and emission lifetime measured on an Edinburgh Instruments FLS980 photoluminescence spectrometer, and their particle morphology analysed by either a Joel 2010 transmission electron microscope or a Joel 2100 transmission electron microscope. 


\section{1b. Results and Discussion of Open-Air Synthesised CsPbBr3-AHpAC}

\section{Observations}

Neither caesium carbonate $\left(\mathrm{Cs}_{2} \mathrm{CO}_{3}\right)$ or any caesium halide salt $(\mathrm{CsCl}, \mathrm{CsBr}$, or $\mathrm{CsI})$ dissolved into toluene, even after one hour of heating at $110^{\circ} \mathrm{C}$. The resulting caesium source is a fine white suspension within a clear and colourless solvent.

The dissolution of either lead chloride $\left(\mathrm{PbCl}_{2}\right)$ or lead bromide $\left(\mathrm{PbBr}_{2}\right)$ in dimethylformamide (DMF) resulted in a clear and colourless solution. The dissolution of lead iodide $\left(\mathrm{PbI}_{2}\right)$ into the same solvent produced a yellow, but clear, solution. In the course of this study it was observed that only caesium lead tribromide nanocrystals $\left(\mathrm{CsPBr}_{3} \mathrm{NCs}\right)$ could be successfully synthesised via this method Multiple attempts to synthesise standard caesium lead trichloride nanocrystals ( $\left.\mathrm{CsPbCl}_{3} \mathrm{NCs}\right)$ and caesium lead triiodide nanocrystals $\left(\mathrm{CsPbI}_{3} \mathrm{NCs}\right)$ all resulted in the formation of white, nonfluorescent precipitates. Due to these failures, the scope of these experiments was limited to $\mathrm{CsPbBr}$ NCs.

The presence of any anthracene based ligand (AC, AEAC, APAC, or AHpAC) within the DMF/ $\mathrm{PbBr}_{2}$ solution resulted in a yellow solution that darkened during the course of the heating process (see

Figure 11h).

Initial experiments aiming to substitute either oleic acid (OA) or oleylamine (OAm) with $A C$ or an AAAC respectively resulted in the formation of a yellow precipitate that did not fluoresce. Therefore, the anthracene based ligands were instead added as additional ligands to the standard reaction mixture. The addition of $\mathrm{AC}$ also resulted in the formation of a non-fluorescent yellow powder, suggesting that AC has a detrimental effect on perovskite formation.

The addition of AEAC or APAC also produced a non-fluorescent yellow solid. A similar result occurred with the addition of AHpAC; however, this precipitate displayed a green fluorescence. Processing and centrifugation of the reaction mixture resulted in the isolation of a non-fluorescent yellow solid and a white solid that fluoresced with an aqua hue (see Figure 11h). The addition of toluene or hexane did not dissolve this solid, indicating that it is not composed of nanoscale particles. 

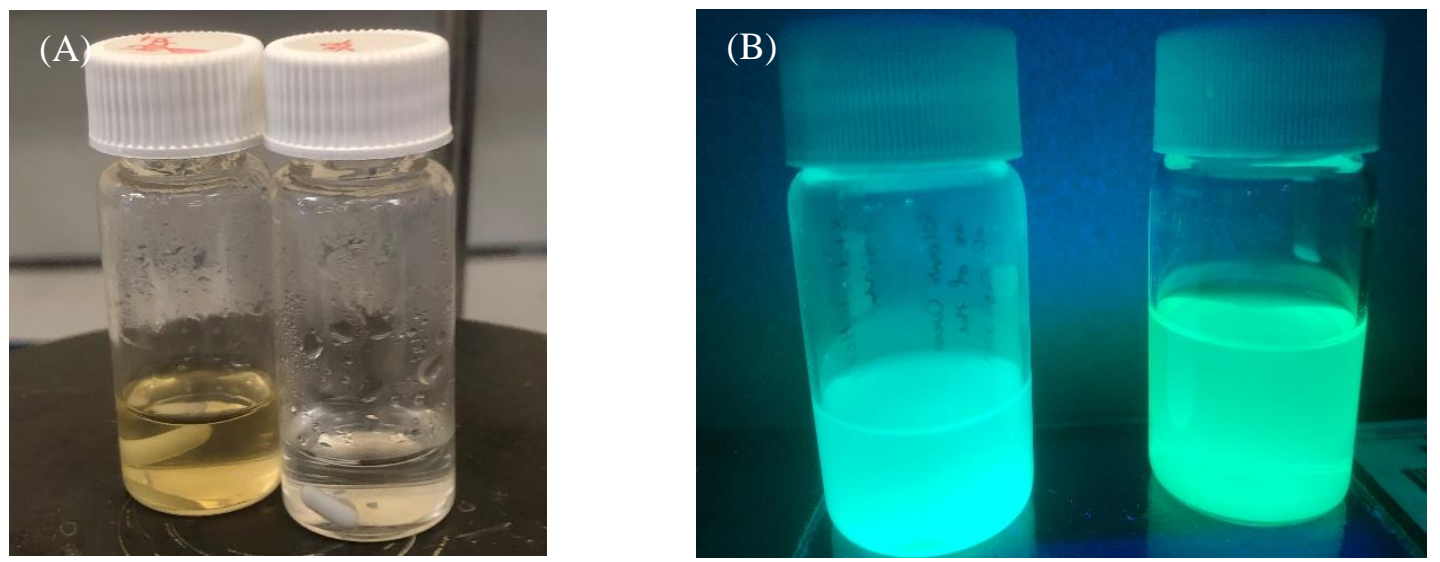

Figure 11h. (A) Right, the standard DMF solution containing $\mathrm{PbBr}_{2}, \mathrm{OA}$, and OAm. Left, the modified DMF solution with AHpAC. (B) Right, the standard CsPbBr $3 \mathrm{NCs}_{3} \mathrm{Left}, \mathrm{Cs} \mathrm{PbBr}_{3}-$ AHpAC.

While the synthesis of both standard $\mathrm{CsPbBr}_{3} \mathrm{NCs}$ and $\mathrm{CsPbBr}_{3}-\mathrm{AHpAC}$ was carried out successfully multiple times, after the last successful synthesis further experiments did not work and instead produced either yellow or white precipitates that did not fluoresce. Therefore, while positive results can be obtained, this method of synthesis proved to be highly unreliable. 


\section{Absorption Measurements of Open-Air Synthesised CsPbBr $-\mathrm{AHpAC}$}

The standard caesium lead tribromide nanocrystals $\left(\mathrm{CsPbBr}_{3} \mathrm{NC}\right.$ 's) display the expected absorption spectrum (see Figure 11j). At the onset of absorption at around $450 \mathrm{~nm}$ is the presence of a peak. The location of such a peak at the absorption onset matches the characteristics on an excitonic peak. After this peak, the absorption steadily increases due to the excitonic continuum. There are additional shoulder peaks in the spectrum which would be the result of various nanoparticle sizes present within the sample, indicating that the size distribution varies slightly.

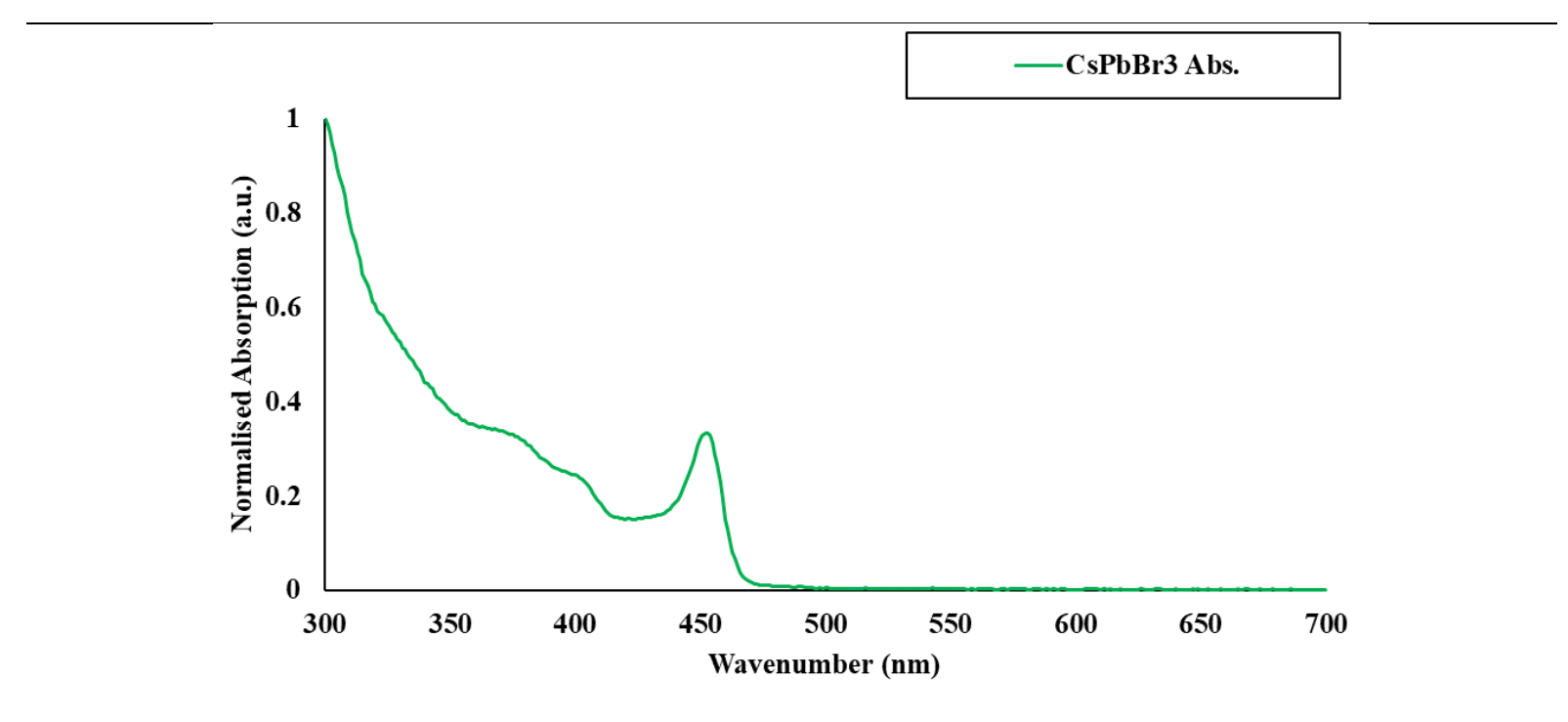

Figure 11j. Absorption Spectrum of standard open-air synthesised $\mathrm{CsPBr}_{3} \mathrm{NCs}$.

While the standard nanocrystals do not absorb at wavelengths below $\sim 475 \mathrm{~nm}$, the modified nanocrystals display significant scattering across the entire range of wavelengths scanned. Such scattering would be due to the larger size of the particles that make up this sample. Despite the significant optical scattering, there is a broad absorption band at $\sim 450 \mathrm{~nm}$ which correlates well with the assumed exciton absorption peak demonstrated by standard nanocrystals, suggesting that this feature may also an excitonic peak (see Figure 11k). 


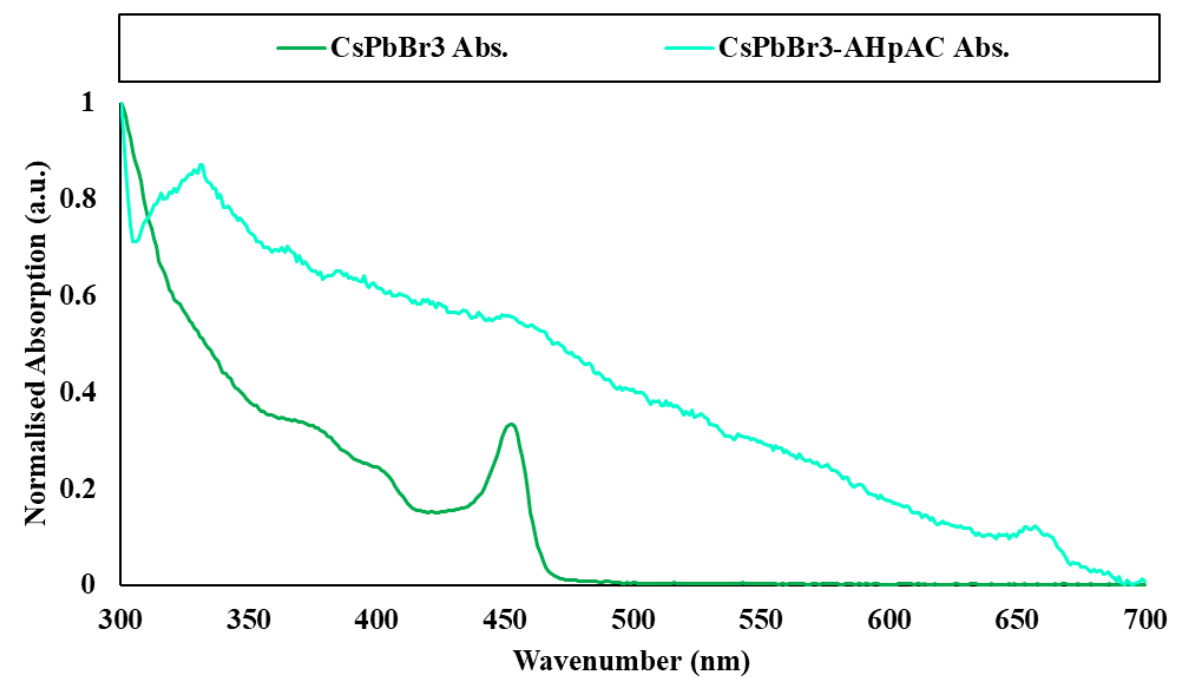

Figure 11k. Comparison between the absorption (Abs.) spectrum of standard CsPbBr $3 \mathrm{NC}^{\prime}$ and $\mathrm{CsPbBr}_{3}-\mathrm{AHpAC}$. (B) Comparison between the absorption spectrum of $\mathrm{N}$-(7-aminoheptyl)anthracen-9-carboxamide (AHpAC).

The sample also displays two small but noticeable broad absorption bands at $\sim 387 \mathrm{~nm}$ and $\sim 364 \mathrm{~nm}$ that correlate well with the two most prominent absorption peaks of AHpAC (see Figure 11l.).

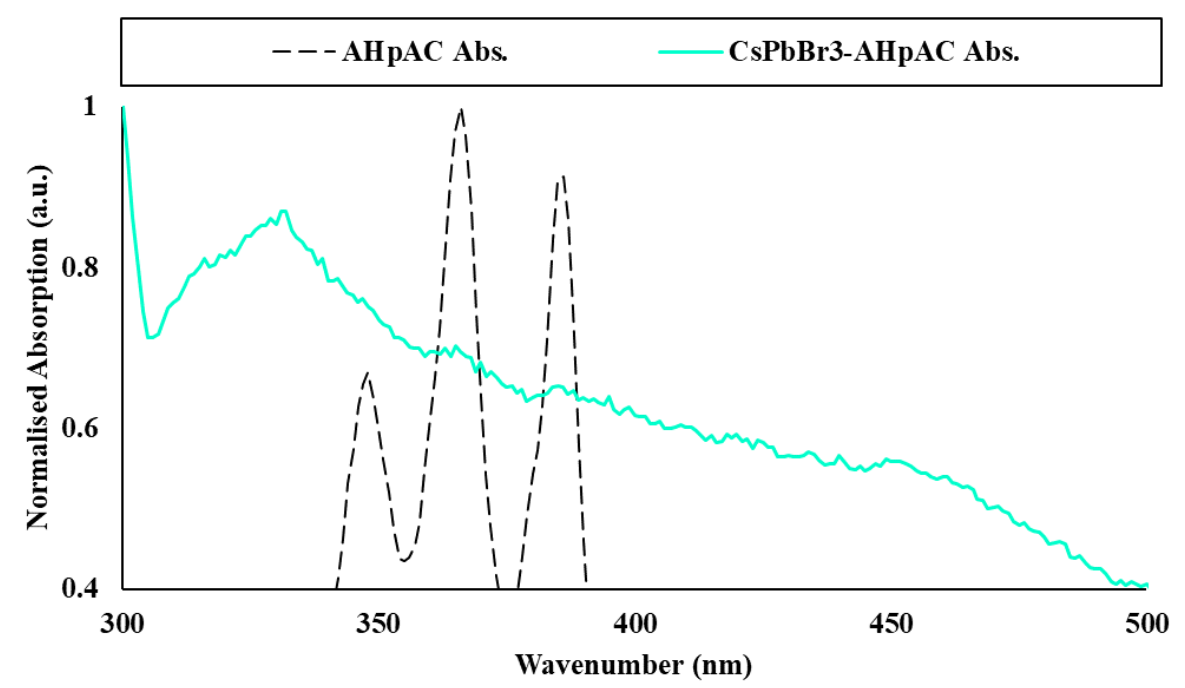

Figure 11l. Comparison between the absorption spectrum of N-(7-aminoheptyl)-anthracen-9carboxamide (AHPAC).

Additionally, the absorption spectrum of the sample also possesses a large, broad peak at $332 \mathrm{~nm}$. A similar absorption band is also observed in the absorption spectrum of the thin-film samples $\mathrm{PbCl}_{2}+\mathrm{ACEACl}$, and there is a strong correlation between the $\sim 330 \mathrm{~nm}$ absorption peaks of two samples (see Figure 11m.). For the thin-film samples, these absorption features were determined to be the result 
of photoinduced dimerization of neighbouring anthracene functional groups. A similar set of possesses could be occurring in the $\mathrm{CsPbB} 3_{3}-\mathrm{AHpAC}$ sample.

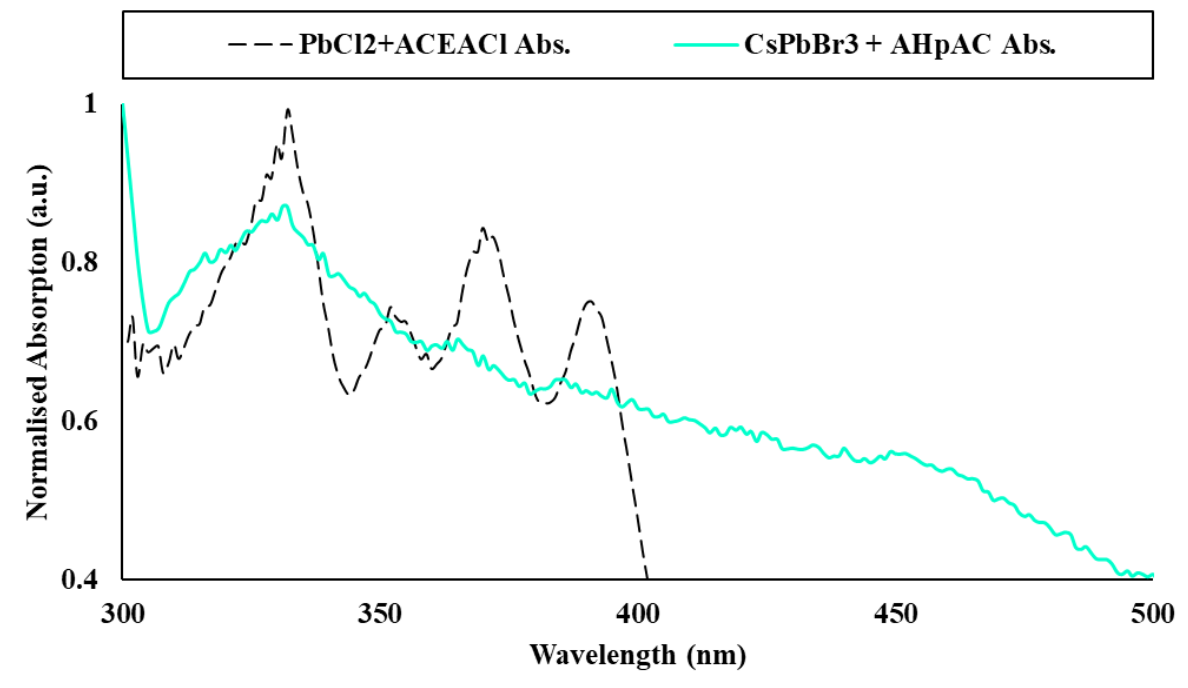

Figure 11m. Close up comparison between the absorption spectra of $\mathrm{CsPBr}_{3}-\mathrm{AHpAC}$ and the thin-film sample $\mathrm{PbCl}_{2}$-ACEACl. Green highlight indicates positive peak correlations.

In summary the electronic absorption spectrum of the open-air synthesised $\mathrm{CsPBBr}_{3}-\mathrm{AHpAC}$ sample shows that this sample highly scatters optical and UV light. Even so, the spectrum does show weak signals associated with both a $\mathrm{CsPBr}_{3}$ phase and an $\mathrm{AHpAC}$ phases.

\section{Photoluminescence of Open-Air Synthesised CsPbBr 3 -AHpAC}

The emission of $\mathrm{CsPbBr}_{3}-\mathrm{AHpAC}$ is extremely similar to that of the standard $\mathrm{CsPbBr}_{3}$ nanocrystals except for a blue-shift of the peak emission wavelength of $16 \mathrm{~nm}$ and a broadening of the emitted wavelengths (see Figure 11n.). Additionally, there is a small but significant amount of emission between 400 and $450 \mathrm{~nm}$. Though the shape of this high energy emission band does not match well with pure AHpAC emission, this band does correlate well with anthracene excimer emission. Excimer formation within this sample is highly likely to occur since it is composed of a fine powder. The high concentration of anthracene functional groups in the solid state, or concentrated around the surface of a nanocrystal, would provide the optimal conditions for excimer formation. ${ }^{70} \mathrm{~A}$ similar display of excimer emission is also observed in many of the $\mathrm{PbX}_{2}$-ACAAX thin-films. 


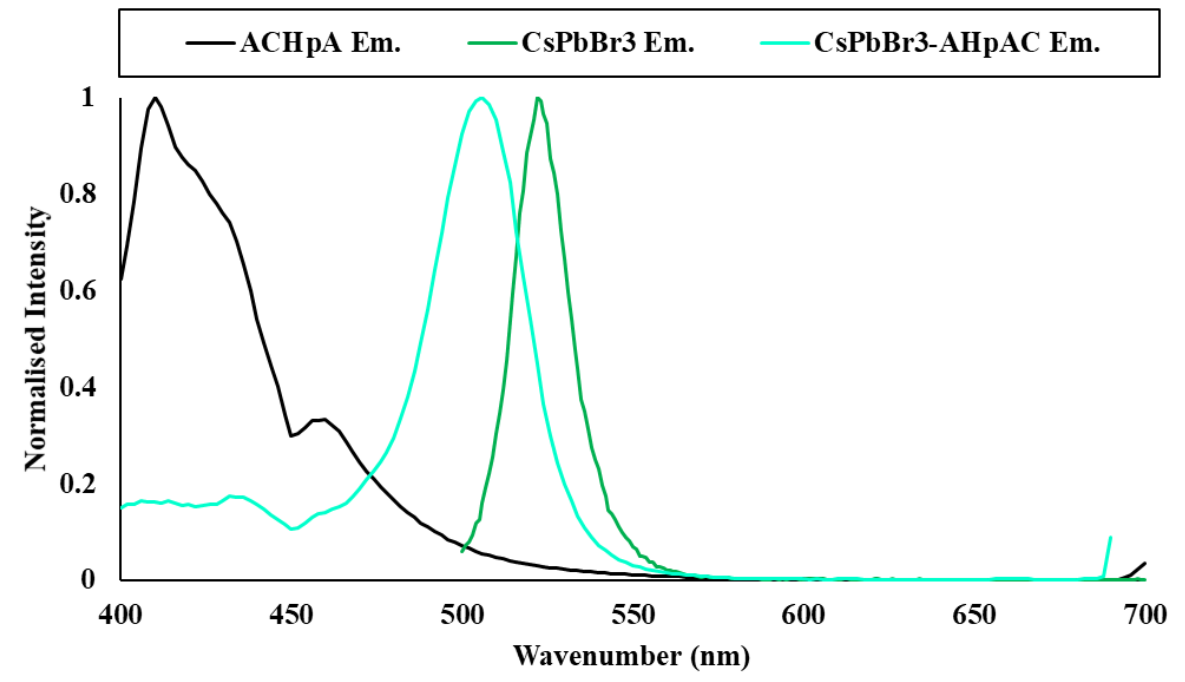

Figure 11n. Electronic emission spectra of standard $\mathrm{CsPbBr}_{3} \mathrm{NCs}$, pure $\mathrm{AHpAC}$, and $\mathrm{CsPbBr}_{3^{-}}$ AHрAC.

After a year of storage in a sealed vial with anhydrous toluene, the sample still fluoresced, indicating a high level of stability. Re-analysis of this sample showed similar perovskite emission of $514 \mathrm{~nm}$, a slight redshift indicating particle growth via aggregation. Additionally, the high energy emission peak has blue-shifted and more strongly resembles monomer emission. This suggests that the anthracene present within the sample has dispersed into the solvent and is unable to form excimers (see Figure 11o).

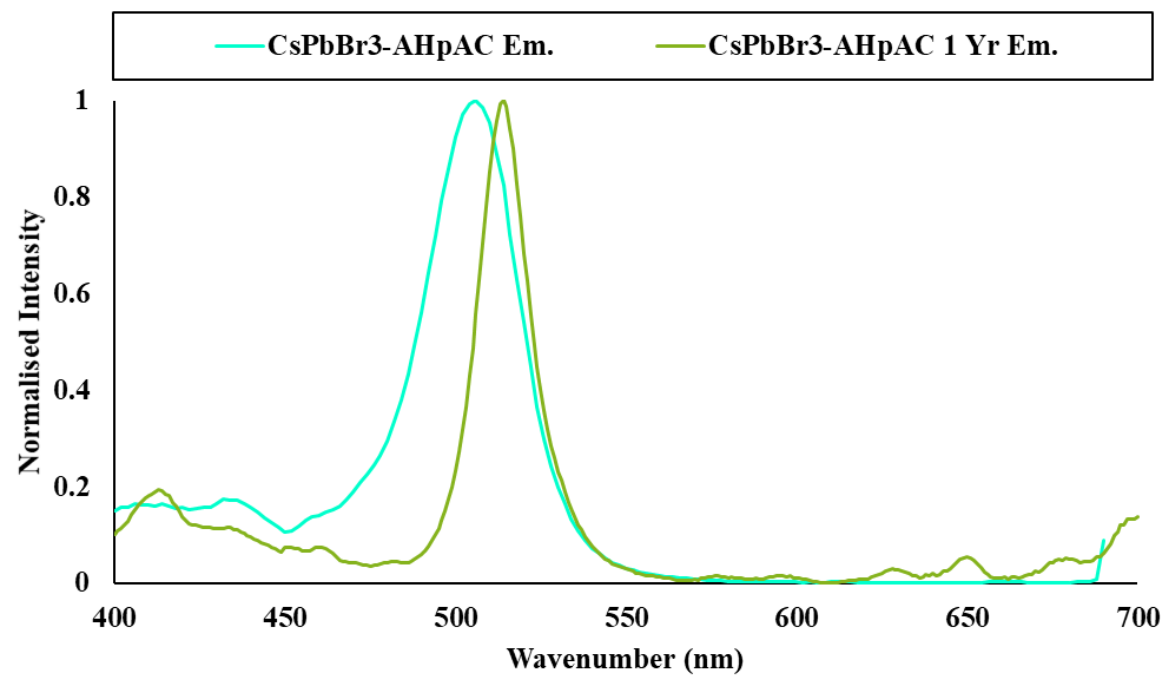

Figure 11o. Electronic emission spectra of standard $\mathrm{CsPbBr}_{3} \mathrm{NCs}$, pure $\mathrm{AHpAC}$, and $\mathrm{CsPbBr}_{3^{-}}$ AHpAC. Green highlight indicates positive peak correlation.

Overall, the electronic emission spectrum of $\mathrm{CsPbBr}_{3}-\mathrm{AHpAC}$ shares emissive features with both standard $\mathrm{CsPbBr}_{3}-\mathrm{NC}$ 's and $\mathrm{AHpAC}$. The presence of anthracene emission shows that at least some of 
the excitation events that are being generated within the molecule is not being transferred to the perovskite phase and is instead resulting in excimer formation and emission. Therefore, any potential energy transfer cannot be $100 \%$ efficient. Additionally, the intensity of anthracene emission is found to increase over time, signalling the loss of coordinated anthracene based ligands over time.

\section{Electronic Excitation of Open-Air Synthesised $\mathrm{CsPbBr}_{3}-\mathrm{AHpAC}$}

The electronic excitation spectrum of both the standard and modified open-air synthesised nanoparticles display rather unique excitation spectra. Most perovskite nanocrystals demonstrate a peak at the low energy onset of excitation, followed by a gradually increasing intensity with decreasing wavelength. While both open-air samples demonstrate an excitation peak at a long wavelength of $467 \mathrm{~nm}$, the intensity does not increase with decreasing wavelength. Instead, for the standard nanoparticles, the intensity stays constant until $\sim 350 \mathrm{~nm}$ after which is rapidly decreases (see Figure 11p.).

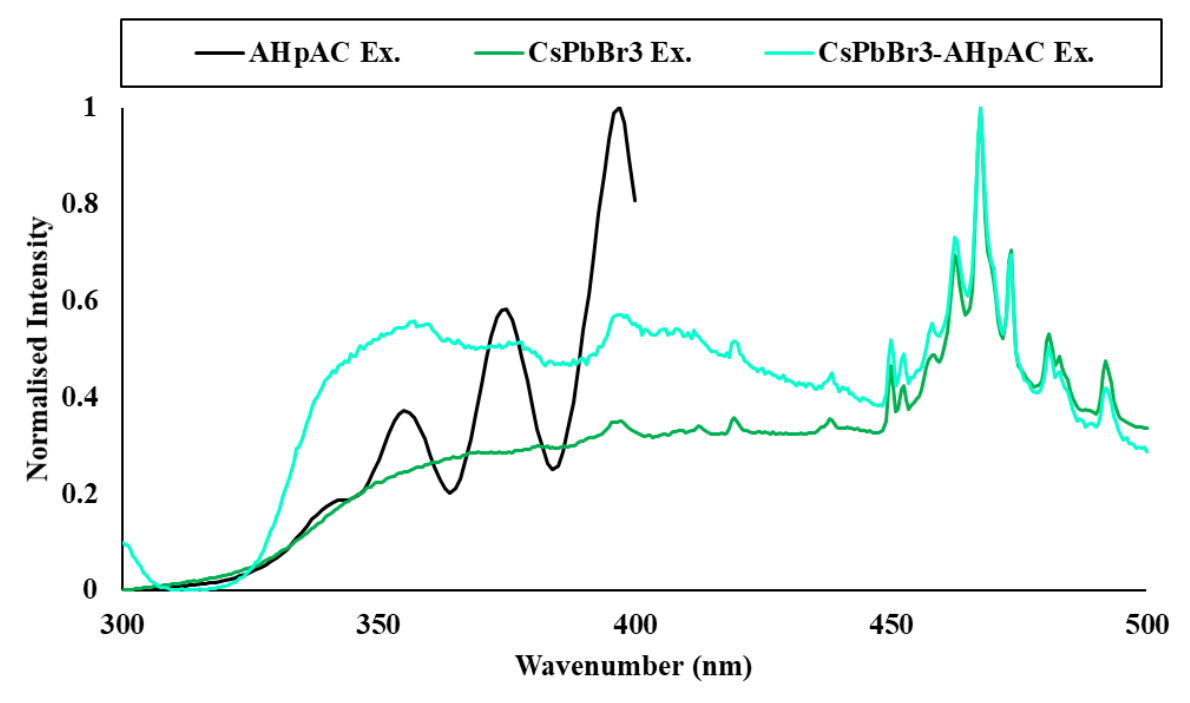

Figure 11p. Comparison between the excitation spectra of standard $\mathrm{CsPbr}_{3} \mathrm{NC}$ 's, pure $\mathrm{AHpAC}$, and $\mathrm{CsPBr}_{3}-\mathrm{AHpAC}$. The scan for $\mathrm{AHpAC}$ ends at $400 \mathrm{~nm}$ due to peak emission at $\sim 410 \mathrm{~nm}$.

The best explanation for this is that the sample was too concentrated. The absorption spectrum shows that both samples principally absorb at this high energy region. The high concentration of these samples would have made them highly opaque to these high energy wavelengths and therefore prevented the bulk of sample from being excited. Therefore, the bulk of the sample is going to fluoresce due to wavelengths that are not as strongly absorbed by the material, explaining the collected excitation spectrum.

The electronic excitation spectrum of the $508 \mathrm{~nm}$ emission of $\mathrm{CsPbBr}_{3}-\mathrm{AHpAC}$ correlates well with that of the standard $\mathrm{CsPbBr}_{3}$ sample. However, unlike the standard $\mathrm{CsPbBr}_{3}$, the excitation spectrum of $\mathrm{Cs} \mathrm{PbBr}_{3}-\mathrm{AHpAC}$ displays a heightened intensity between 325 and $450 \mathrm{~nm}$. This excitation band 
possesses three broad peaks which correlate well with the excitation peaks of pure AHpAC (see Figure 11p.). Therefore, based on these results, it can be concluded that AHpAC is absorbing and transferring energy to the perovskite phase.

However, while energy transfer is able to be performed between anthracene and the inorganic semiconductor, overlaying the excitation spectrum of $\mathrm{CsPbBr}_{3}-\mathrm{AHpAC}$ with the related absorption spectrum shows that there is a negative correlation between the anthracene peaks (see Figure 11q). As discussed previously, the anthracene absorption peaks identified in $\mathrm{CsPbBr}_{3}-\mathrm{AHpAC}$ correlate well with those displayed by pure AHpAC (refer back to Figure 11l).

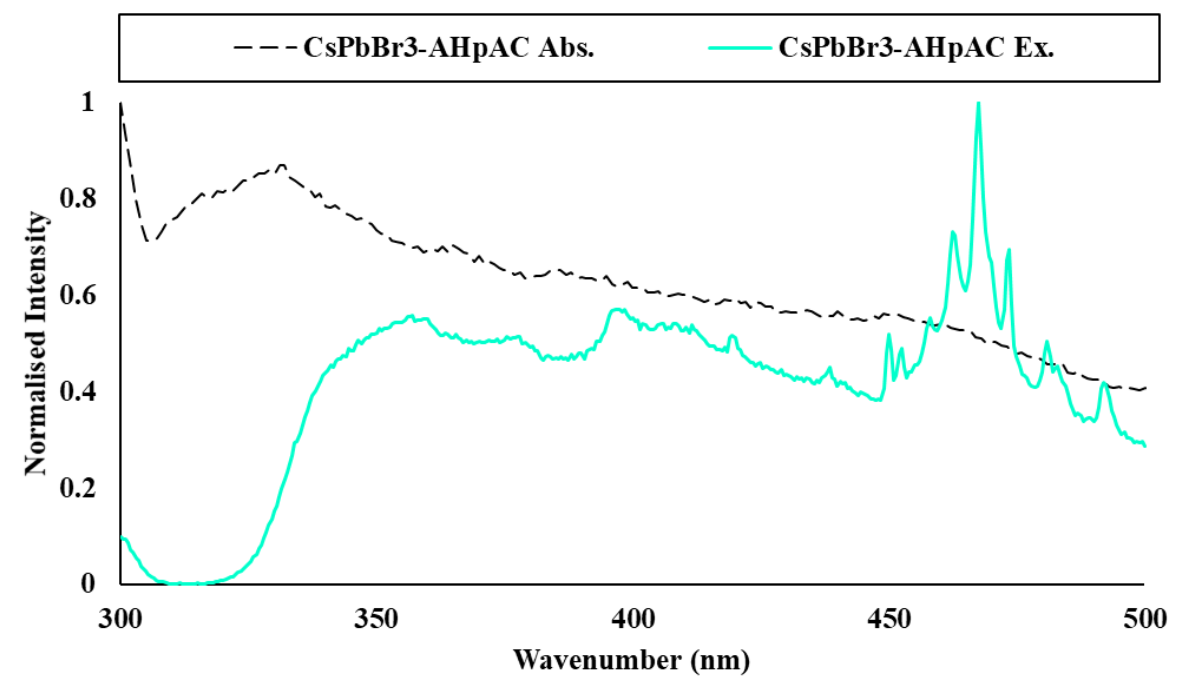

Figure 11q. Comparison between the electronic absorption spectrum of $\mathrm{CsPBBr}_{3}-\mathrm{AHpAC}$ and the electronic excitation spectra of $\mathrm{CsPbBr}_{3}-\mathrm{AHpAC} 508 \mathrm{~nm}$ emission.

Excitation measurements of the high energy emission feature at $436 \mathrm{~nm}$ produce an excitation spectrum that correlates well with the identified anthracene absorption peaks of $\mathrm{CsPbBr}_{3}-\mathrm{AHpAC}$ (see Figure $11 r$ ). Therefore, peak anthracene absorption primarily results in anthracene emission, while non-peak absorption is at least partially transferred to the perovskite as indicated by the increased excitation intensity between 325 and $450 \mathrm{~nm}$. This would suggest that the concentration of anthracene is too high and is preventing the efficient harvesting of these peak wavelengths. Or it suggests that there is not a great enough driving force to facilitate efficient energy transfer. Such a driving force would need to be supplied by either a sharp energy gradient between the singlet excited state of anthracene and the conduction band minimum of the nanomaterial, or electron spin resonance. 


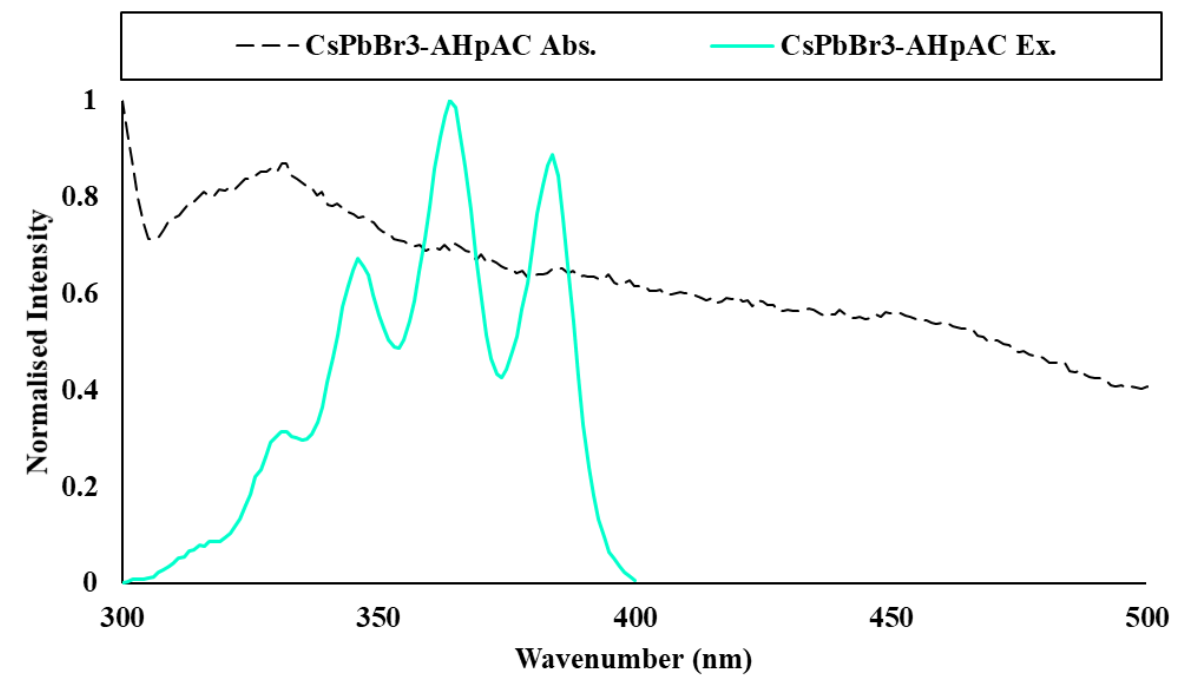

Figure 11r. Comparison between the electronic absorption spectrum of pure AHpAC and the electronic excitation spectra of $\mathrm{Cs}_{\mathrm{PbBr}}-\mathrm{AH} \mathrm{HAC} 414 \mathrm{~nm}$ emission.

In addition to the electronic emission of the sample, the excitation wavelengths of $\mathrm{CsPbBr}_{3}-\mathrm{AHpAC}$ were re-analysed after a year of storage. While the emission was highly similar, the excitation is significantly different. The excitation peak at $467 \mathrm{~nm}$, which was associated with exciton formation, is gone. Additionally, the intensity of emission due to 300 to $400 \mathrm{~nm}$ light has significantly increased in relation to post $400 \mathrm{~nm}$ wavelengths (see Figure 11s). This excitation spectrum correlates extremely well with those of $\mathrm{Cs}_{4} \mathrm{PbBr}_{6}$, as reported by Zhang et al. ${ }^{71}$

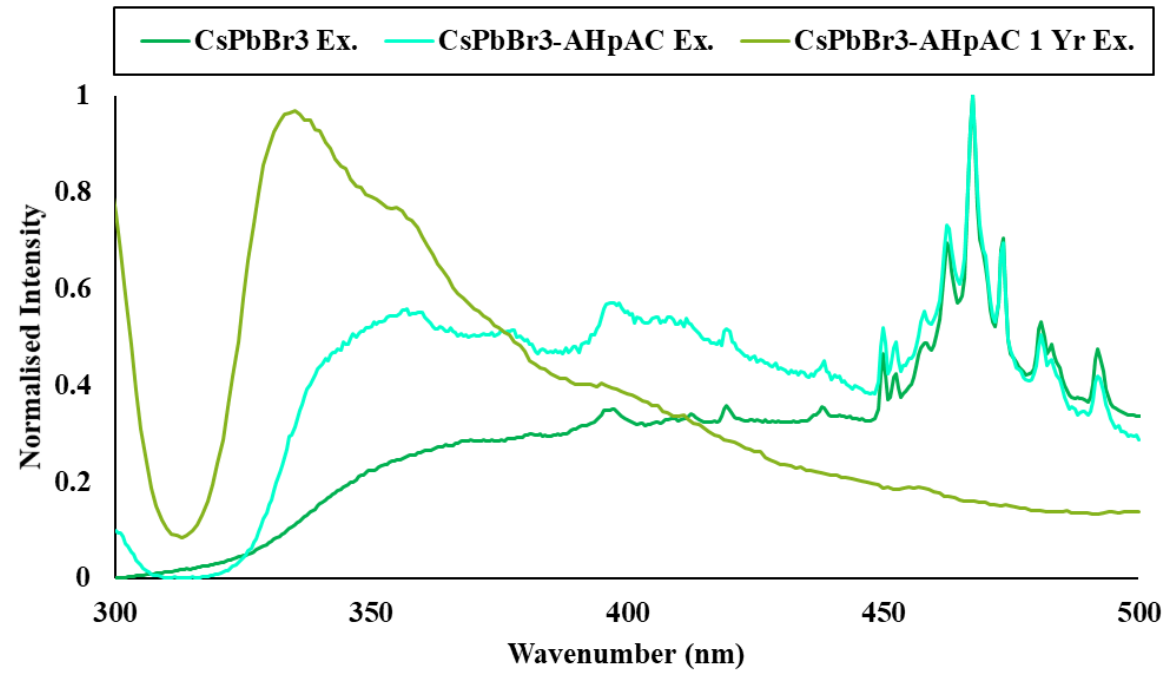

Figure 11s. Comparison between the excitation spectra of standard $\mathrm{CsPBBr}_{3} \mathrm{NCs}, \mathrm{CsPbBr}_{3}-$ $A H p A C$, and $\mathrm{Cs} \mathrm{PbBr}_{3}-\mathrm{AH} \mathrm{HAC}$ after one year of storage. 
Due to limited time, further analysis of this sample could not be performed, however, the correlation between this latest excitation spectrum and those of the literature provide strong evidence for the transformation of three dimensional $\mathrm{CsPbBr}_{3}$ to zero dimensional $\mathrm{Cs}_{4} \mathrm{PbBr}_{6}$ due to possible metastability of the original product or a lead extraction process. Comparing the one year excitation measurement with that of pure AHpAC shows that the former still demonstrates small peaks that correlate well with those in the excitation spectrum of the later. Therefore, here presented is evidence that anthracene functional groups are capable of transferring energy to both three dimensional perovskites and zero dimensional perovskites.

\section{Photoluminescence Lifetime of Open-Air Synthesised CsPbBr 3 -AHpAC}

Comparing the emission decay of freshly synthesised $\mathrm{CsPbBr}_{3}-\mathrm{AHpAC} 508 \mathrm{~nm}$ emission with that of standard $\mathrm{CsPbBr}_{3}$ shows an increase in the emission lifetime for the modified material (see Figure 11t.)

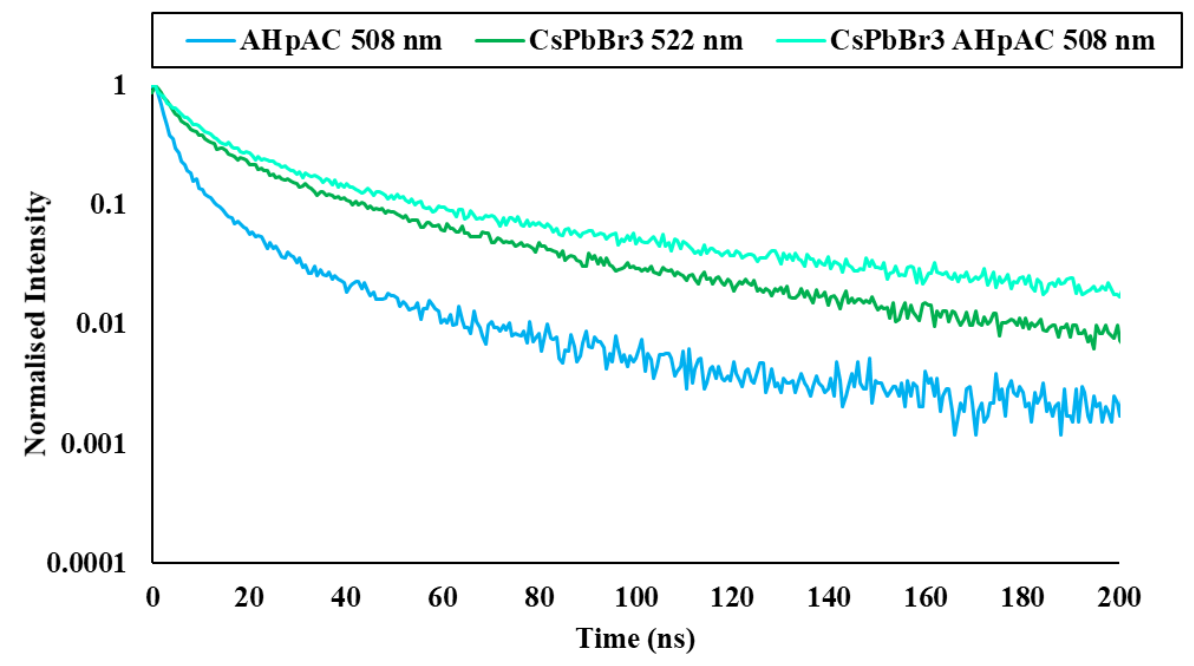

Figure 11t. Comparison between the $\sim 500 \mathrm{~nm}$ emission of pure $\mathrm{AHpAC}$, standard $\mathrm{CsPbBr}{ }_{3} \mathrm{NCs}$, and $\mathrm{Cs}_{\mathrm{PbBr}}-\mathrm{AH} \mathrm{HAC}$.

Additionally, the lifetime for anthracene emission at $\sim 410 \mathrm{~nm}$ is reduced when compared to pure AHpAC (see Figure 11u). This is another line of evidence that supports the conclusion that energy is being transferred from the anthracene functional groups to the perovskite phase. Taking the log of the normalised intensity values show that the rate of decay for the emission of this sample is, like that of the standard $\mathrm{CsPbBr}_{3}$, multi-exponential and implying an energy cascade system. 


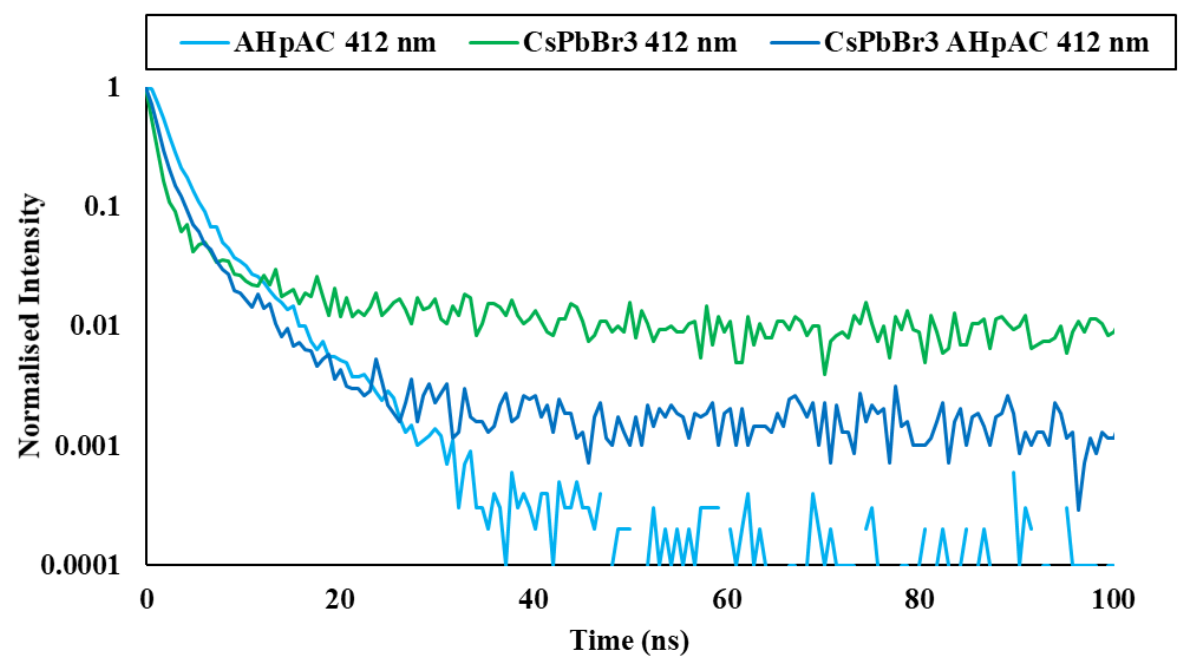

Figure 11u. Comparison between the $412 \mathrm{~nm}$ emission lifetime of pure $\mathrm{AHpAC}$, standard CsPbBr $\mathrm{NCs}$, and $\mathrm{Cs}_{\mathrm{PbBr}} \mathrm{-AHpAC}$. Reference refers to the instrument response.

Photoluminescence Quantum Yield of Open-Air Synthesised CsPbBr 3 -AHpAC

Standard open-air synthesised $\mathrm{CsPbBr}_{3} \mathrm{NCs}$ displayed a photoluminescence quantum yield (PLQE) of $53.76 \%$ when exciting at both 467 and $337 \mathrm{~nm}$. Considering that caesium lead trihalide are capable of PLQE values beyond 90\%, this would suggest that the surfaces of these nanocrystals have not been well passivated by the ligands and possess many surface defects that act as sites for trap-assisted recombination. The modified $\mathrm{CsPbBr}_{3}-\mathrm{AHpAC}$ displays a similar PLQE of 53.64\% when exciting at $467 \mathrm{~nm}$. However, when exciting the sample at $337 \mathrm{~nm}$, the PLQE drops to $28.64 \%$, representing a 53\% drop in efficiency. This result further confirms that the energy transfer process between AHpAC and the perovskite phase is not $100 \%$ efficient. Therefore, less perovskite emission is being gained due to anthracene excitation when compared to directly exciting the perovskite.

\section{Transmission Electron Microscope Images of Open-Air Synthesised CsPbBr 3 -AHpAC}

Analysis of $\mathrm{CsPbBr}_{3}-\mathrm{AHpAC}$ shows that the majority of the sample is composed of large, micrometre scale particles (refer to Figure 11v). While most of the sample lacks clear lattice fringing and therefore appears to be amorphous, there are smaller regions that do display lattice fringing and therefore are crystalline. There is also within the sample smaller $10-20 \mathrm{~nm}$ features. These features were highly sensitive to the electron beam and therefore could not be viewed under high magnification without disintegrating. However, it is highly likely that these particles are individual caesium lead tribromide nanocrystals. 


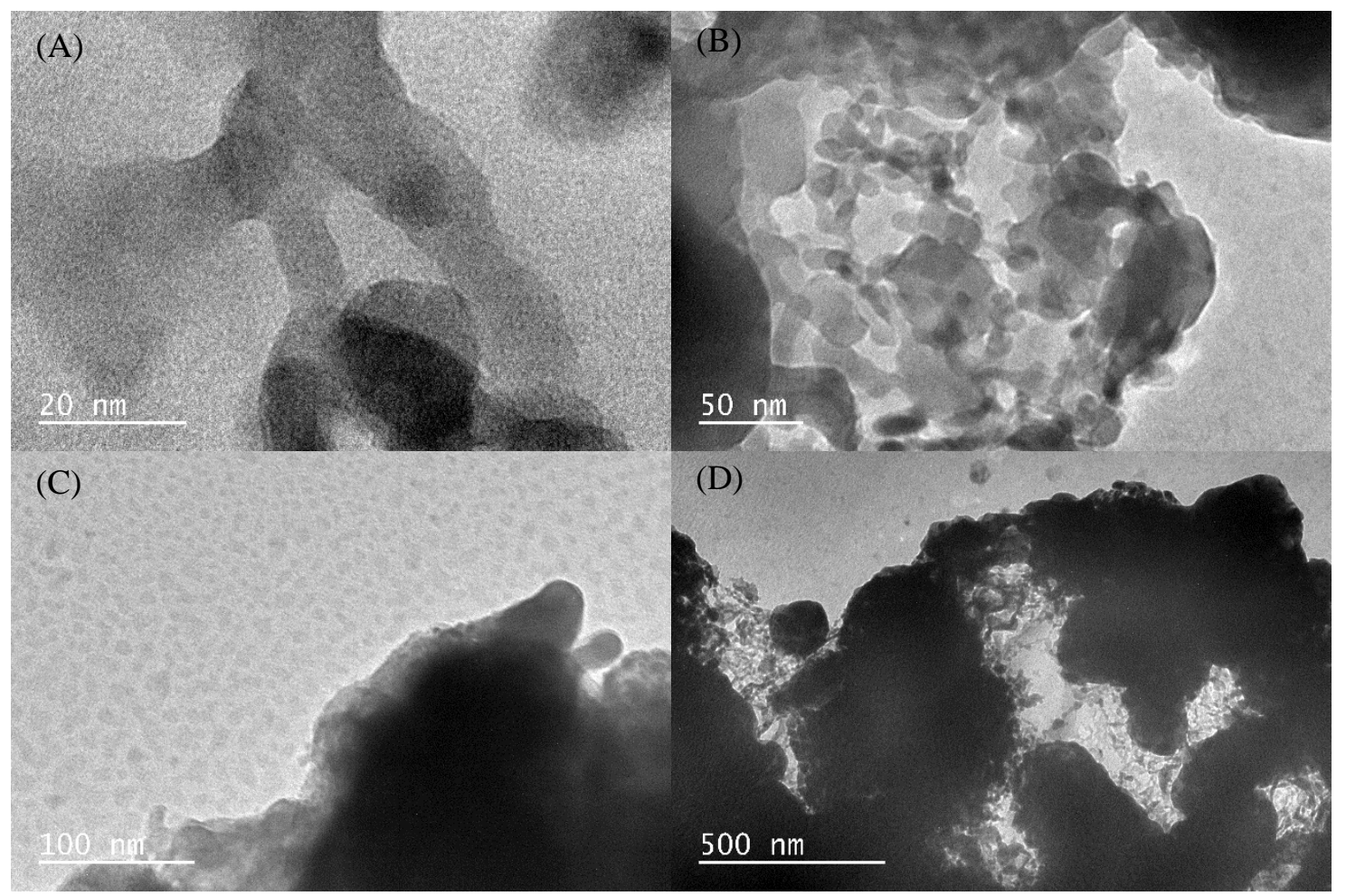

Figure 11v. TEM images of open-air synthesised $\mathrm{CsPBr}_{3}-\mathrm{AHpAC}$.

The larger scale, amorphous features are likely to be composed of degraded semiconductor and aggregated AHpAC. The clear separation between the amorphous and crystalline/nanoparticle phases would help to explain the low efficiency of the energy transfer. The majority of the AHpAC phase is likely to be outside of the FRET radius of a nanoparticle and are therefore detrimental to the performance of the perovskite.

Based upon these images and the observations of the synthesis of these particles, the inclusion of AHpAC appears to facilitate the aggregation and possible degradation of $\mathrm{CsPbr}_{3}$ nanoparticles into an amorphous phase. Such large particles significantly reduce the transmittance of solution, and acts as a sink for 332, 365, and $385 \mathrm{~nm}$ light.

\section{1c. Concluding Remarks on Open-Air Synthesised CsPbBr3-AHpAC}

The inclusion of $\mathrm{AHpAC}$ in the reaction mixture of $\mathrm{CsPbr}_{3}$ is overall detrimental to the stabilisation of a nanocrystal solution by facilitating aggregation and possible degradation of the nanoparticles. However, despite this aggregation, some samples did fluoresce, and analysis of these samples showed that energy transfer does occur between anthracene and the perovskite phase. This energy transfer is based upon the positive correlation between the excitation spectra of the sample to that of pure AHpAC, and the increase in the lifetime of the characteristic perovskite emission. 
However, re-analysis of these samples, specifically their excitation spectrum, had shown that after one year the samples had degraded into what is suspected to be zero-dimensional $\mathrm{Cs}_{4} \mathrm{PbBr}_{6}$. While this can be detrimental, Akkerman et al has shown that zero-dimensional $\mathrm{Cs}_{4} \mathrm{PbBr}_{6}$ can be converted into threedimensional $\mathrm{CsPbBr}_{3}$, providing a potential method to restore the original materials and the associated properties. ${ }^{72}$ However, this method of nanocrystal synthesis has proved to be highly unreliable and narrow in scope. Therefore, these results could not be replicated for caesium lead trichloride or caesium lead triiodide.

Therefore, as with the thin-film and intercalation samples, high concentrations of AAAC is detrimental to both perovskite formation and performance. However, the presence of minor energy transfer between an anthracene based molecule and a lead halide perovskite does demonstrate a useful coupling between the two phases that was not demonstrated in those projects and opens up the possibility for more optimised couplings. 


\section{Coordination of Anthracene Based Ligands to Hot-Injection Synthesised $\mathrm{CsPbX}_{3}$ Nanocrystals}

\section{2a. Hot-Injection Synthesis of $\mathrm{CsPbX}_{3}$ Nanocrystals}

A more conventional and well established air free synthesis of caesium lead halide nanocrystals was experimented with in attempts to incorporate anthracene based ligands (ALs) onto the surface of the nanoparticles. The hot injection method used was based upon that developed by Protesescu et al (2015) and requires the preparation of a stock solution of caesium oleate, a portion of which is injected into a mixture of lead halide salt, oleic acid (OA), oleylamine (OAm) and 1-octadecene (ODE), allowing for the precipitation and stabilisation of colloidal $\mathrm{CsPbX}_{3}$ nanocrystals. ${ }^{31}$

\section{Hot-injection Method Step 1: Preparation of Caesium Oleate}

$0.814 \mathrm{~g}(2.50 \mathrm{mmol})$ of caesium carbonate $\left(\mathrm{Cs}_{2} \mathrm{CO}_{3}\right)$ was added to a $100 \mathrm{~mL}$ three neck round bottom flask along with $40 \mathrm{~mL}$ of ODE and $2.5 \mathrm{~mL}$ of OA. Using a vacuum line, the reaction mixture was degassed under vacuum at $120^{\circ} \mathrm{C}$ for one hour. Afterwards an atmosphere of dry nitrogen $\left(\mathrm{N}_{2}\right)$ was introduced to the reaction vessel and the temperature raised to $150^{\circ} \mathrm{C}$ for one hour or until all of the $\mathrm{Cs}_{2} \mathrm{CO}_{3}$ has reacted with the OA (see Figure 11a) for reaction details). The product solution was then cooled to $100{ }^{\circ} \mathrm{C}$ and utilised to produce caesium lead trihalide nanocrystals.

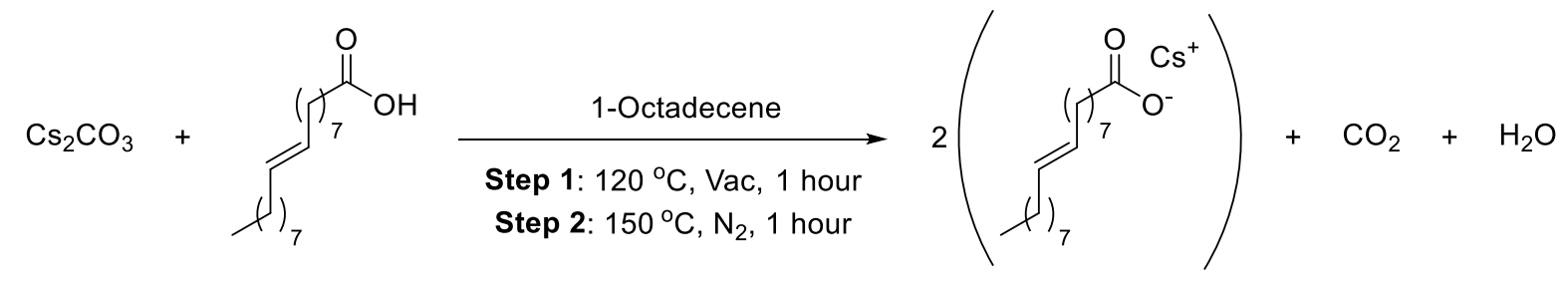

\begin{tabular}{|c|c|c|c|c|c|}
\hline Compound & $\begin{array}{c}\text { Molar } \\
\text { Equivalents }\end{array}$ & mmol & Mass/Volume & $\begin{array}{l}\text { Density } \\
\left(\mathrm{g} \mathrm{mL}^{-1}\right)\end{array}$ & $\begin{array}{c}\text { Molar } \\
\text { Mass } \\
\left(\text { g mol }^{-1}\right)\end{array}$ \\
\hline $\mathrm{Cs}_{2} \mathrm{CO}_{3}$ & 1 & 2.5 & $814 \mathrm{mg}$ & - & 325.82 \\
\hline Oleic Acid & 3.17 & 7.92 & $2.5 \mathrm{~mL}$ & 0.895 & 282.47 \\
\hline Octa-1-decene & 50 & 125 & $40 \mathrm{~mL}$ & 0.789 & 252.49 \\
\hline
\end{tabular}




\section{Hot-injection Method Step 2: Preparation of Anthracene Stabilised Caesium Lead Halide}

\section{Nanocrystals}

$0.188 \mathrm{mmol}$ of $\mathrm{PbX}_{2}(\mathrm{X}=\mathrm{Br}$, I, or a mixture of two $)$ was placed into a $25 \mathrm{~mL}$ three neck round bottom flask. $5 \mathrm{~mL}$ of ODE was added to the vessel along with $0.5 \mathrm{~mL}$ of OA and $0.5 \mathrm{~mL}$ of OAm. The reaction mixture must be placed under vacuum using a vacuum line and heated to $120^{\circ} \mathrm{C}$ for one hour. Afterwards the reaction mixture was placed under a dry $\mathrm{N}_{2}$ atmosphere and heated to anywhere between $140-200^{\circ} \mathrm{C}$ (depending on the desired nanocrystal size) for the hot injection of caesium oleate (see Figure 12b). After insuring that the caesium oleate stock solution was at $100^{\circ} \mathrm{C}, 0.4 \mathrm{~mL}$ of the solution was injected into the vigorously stirred lead solution to insure rapid mixing of the reagents. Five seconds after injection, the reaction mixture was be cooled by an ice-water bath. See Figure $\mathbf{1 2 b}$ and Table $12 a$ for details.
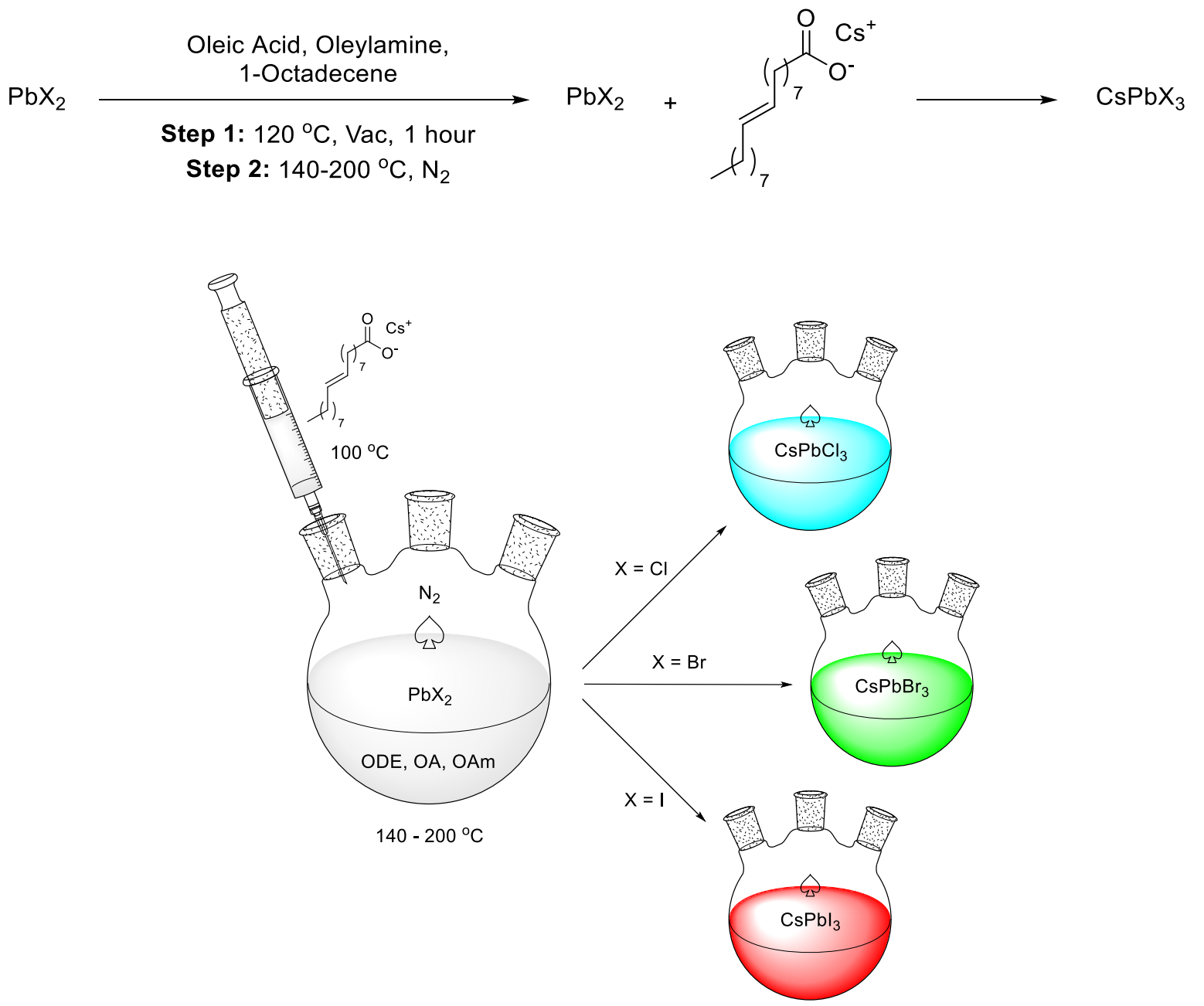

Figure 12b. Synthesis of caesium lead halide nanoparticles. $\mathrm{X}=\mathrm{Cl}, \mathrm{Br}$, or I. 


\begin{tabular}{cccccc}
\hline Compound & $\begin{array}{c}\text { Molar } \\
\text { Equivalents }\end{array}$ & mmol & Mass/Volume & $\begin{array}{c}\text { Density } \\
\left(\mathbf{g ~ m \mathbf { L } ^ { - 1 }}\right)\end{array}$ & $\begin{array}{c}\text { Molar } \\
\text { Mass } \\
\left(\mathbf{g ~ m o l}^{\mathbf{1}}\right)\end{array}$ \\
& & & & & \\
Caesium Source & - & - & $0.4 \mathrm{~mL}$ & - & - \\
$\mathbf{P b C l}_{2}$ & 1 & 0.19 & $52.3 \mathrm{mg}$ & - & 278.10 \\
${\text { or } \mathbf{P b B r}_{2}}_{\text {or PbI }}$ & 1 & 0.19 & $69.0 \mathrm{mg}$ & - & 367.01 \\
Oleic Acid & 1 & 0.19 & $86.7 \mathrm{mg}$ & - & 461.01 \\
Oleylamine & 8.5 & 1.6 & $0.5 \mathrm{~mL}$ & 0.895 & 282.47 \\
1-Octadecene & 8 & 1.5 & $0.5 \mathrm{~mL}$ & 0.813 & 267.49 \\
*Trioctylphosphine & 83 & 15.6 & $5 \mathrm{~mL}$ & 0.789 & 252.49 \\
& 11.9 & 2.2 & $1 \mathrm{~mL}$ & 0.831 & 370.63
\end{tabular}

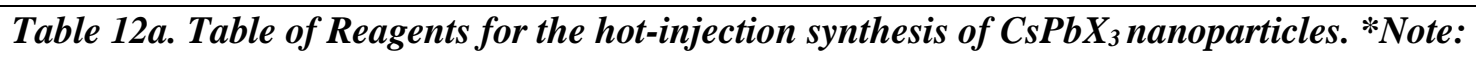

Trioctylphosphine is only required to dissolve $\mathrm{PbCl}_{2}$ for the synthesis of $\mathrm{Cs}_{\mathrm{PbCl}}$.

\section{Hot-injection Method Step 3: Washing Procedure}

After the reaction had been sufficiently cooled, the reaction mixture was transferred to a centrifuge tube, followed by the addition of at least two volumetric equivalents of antisolvent, either $n$-butanol $\left({ }^{n} \mathrm{BuOH}\right)$ or $t$-butanol $\left({ }^{t} \mathrm{BuOH}\right)$. The mixture was spun at $10000 \mathrm{rpm}$ after which the supernatant was discarded and the solid collected. The solid was resuspended in $5 \mathrm{~mL}$ of toluene or hexane, followed by a volumetric equivalent of antisolvent. The mixture was centrifuged at $5000 \mathrm{rpm}$. The solid was collected and resuspended in $5 \mathrm{~mL}$ of toluene or hexane. This process was repeated at least two times. The final nanocrystal solution was spun without antisolvent at between $1500-5000 \mathrm{rpm}$ for 2 mins to separate out increasingly smaller particles from the solution. Each solid sample that was extracted was collected was resuspended in an appropriate amount of solvent to produce a solution with a narrow range of particles sizes. 


\section{$\underline{\text { Modifications }}$}

The hot-injection method was modified by adding anthracene based ligands (ALs) as either additional reagents in the reaction mixture or as replacements for the fatty ligands.

Initial modifications involved replacing OA with anthracene-9-carboxylic acid (AC) and OAm with a $N$-(aminoalkyl)anthracene-9-carboxamide (AAAC) (see Table $\mathbf{1 1 b}$ and Table 11c).

\begin{tabular}{|c|c|c|c|c|c|}
\hline Compound & Equivalents & mmol & Mass/Volume & $\begin{array}{l}\text { Density } \\
\left(\mathrm{g} \mathrm{m} \mathbf{L}^{-1}\right)\end{array}$ & $\begin{array}{l}\text { Molar Mass } \\
\qquad\left(\mathrm{g} \mathrm{mol}^{-1}\right)\end{array}$ \\
\hline $\mathbf{A C}$ & 8.5 & 1.6 & $355.6 \mathrm{mg}$ & - & 222.24 \\
\hline Oleylamine & 8 & 1.5 & $0.5 \mathrm{~mL}$ & 0.813 & 267.49 \\
\hline \multicolumn{6}{|c|}{ 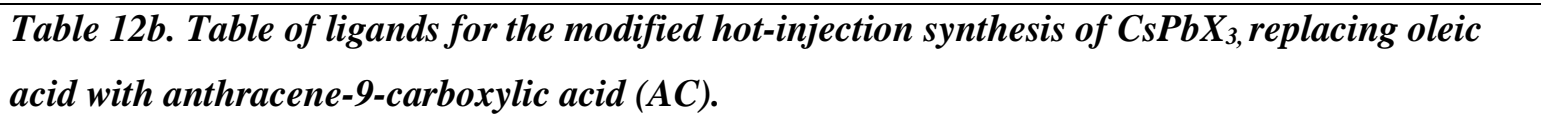 } \\
\hline
\end{tabular}

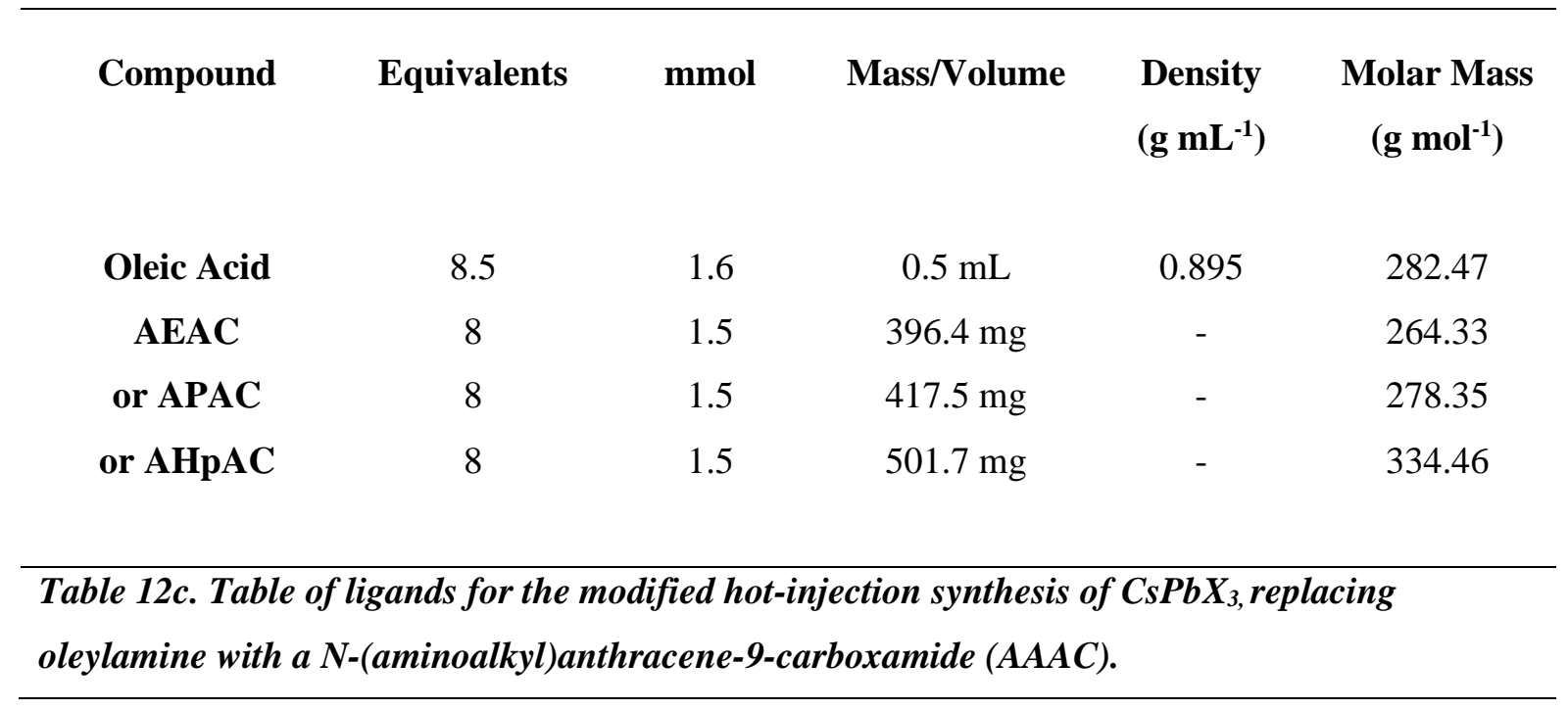

Further experiments added varying molar equivalents of either AC, AEAC, APAC, or AHpAC to the standard reaction mixture.

\section{$\underline{\text { Analysis }}$}

All samples will have their absorption spectrum measured by a Varian Cary 50 Scan UV/Vis Spectrophotometer, their photoluminescence properties including emission, excitation, photoluminescence quantum yield, and emission lifetime measured by an Edinburgh Instruments FLS980 photoluminescence spectrometer, and their particle morphology analysed by either a Joel 2010 transmission electron microscope or a Joel 2100 transmission electron microscope. 


\section{2b. Results of Hot-Injection Synthesised $\mathrm{CsPbX}_{3}$ and $\mathrm{CsPbX}_{3}-\mathrm{AL}$ Nanocrystals}

\section{Observations}

Caesium carbonate $\left(\mathrm{Cs}_{2} \mathrm{CO}_{3}\right)$ slowly dissolved into the 1-octadecene (ODE) solution over the course of the degassing process. During this process, the solution appeared to be refluxing, with the solution bubbling vigorously and a large amount of solvent condensing at the neck of the flask. Placing a nitrogen atmosphere into the reaction vessel stopped the apparent reflux, however, the reaction mixture remained clear and colourless. Repeated degassing of the solution slowly induced a colour change from colourless to yellow and even brown after extensive use.

Even with the addition of the recommended $1 \mathrm{~mL}$ of trioctylphosphine (TOP), the solid lead chloride $\left(\mathrm{PbCl}_{2}\right)$ did not dissolve into the ODE solution. This was a similar problem encountered in the original publication by Protesescu $e t \mathrm{al}^{31}$. As a consequence, the injection of caesium oleate did not result in the formation of a strongly fluorescent solution. Due to time constraints, further attempts to synthesis caesium lead trichloride $\left(\mathrm{CsPbl}_{3}\right)$ were not carried out.

Lead bromide $\left(\mathrm{PbBr}_{2}\right)$ dissolved into the ODE solution as expected, producing a clear and colourless solution. The addition of caesium oleate resulted in an immediate colour change to bright lime green, indicating the successful formation of caesium lead tribromide $\left(\mathrm{Cs}_{\mathrm{PbBr}}\right)$ nanocrystals (see Figure 11a). Quenching of the reaction via the use of a water ice bath quenched the reaction and reduced the chance of the particles degrading and converting in an undesired yellow precipitate.

Lead iodide $\left(\mathrm{PbI}_{2}\right)$ also readily dissolved into the ODE solution, forming a clear yellow solution. Protesescu et al recommends raising the temperature of the $\mathrm{PbI}_{2} / \mathrm{ODE}$ solution to at least $200{ }^{\circ} \mathrm{C}$ before injecting caesium oleate ${ }^{31}$. However, in the course of this work, raising the temperature of the solution past $\sim 150{ }^{\circ} \mathrm{C}$ always resulted in the formation of an insoluble orange lead oxide precipitate. Follow up injection of caesium oleate after the formation of this orange precipitate always resulted in the formation of a black non-fluorescent solid.

To overcome this problem, furthers attempts at synthesising red emitting perovskite nanocrystals involved the substitution of $25 \%$ of the $\mathrm{PbI}_{2}$ with $\mathrm{PbBr}_{2}$ in an attempt to produce a more stable product. However, the reaction mixture would still produce the orange precipitate at the same conditions. However, injection of caesium lead oleate below the less optimal $200{ }^{\circ} \mathrm{C}$ resulted in the formation of a red solution of caesium lead iodide/bromide $\left(\mathrm{CsPb}_{2.25} \mathrm{Br}_{0.75}\right)$ nanocrystals. Lower temperature injection of caesium oleate was also found to work for pure $\mathrm{CsPbI}_{3}$ nanocrystals (see Figure 21c). Experiments were performed on both the iodide/bromide hybrids and pure iodide perovskites. 


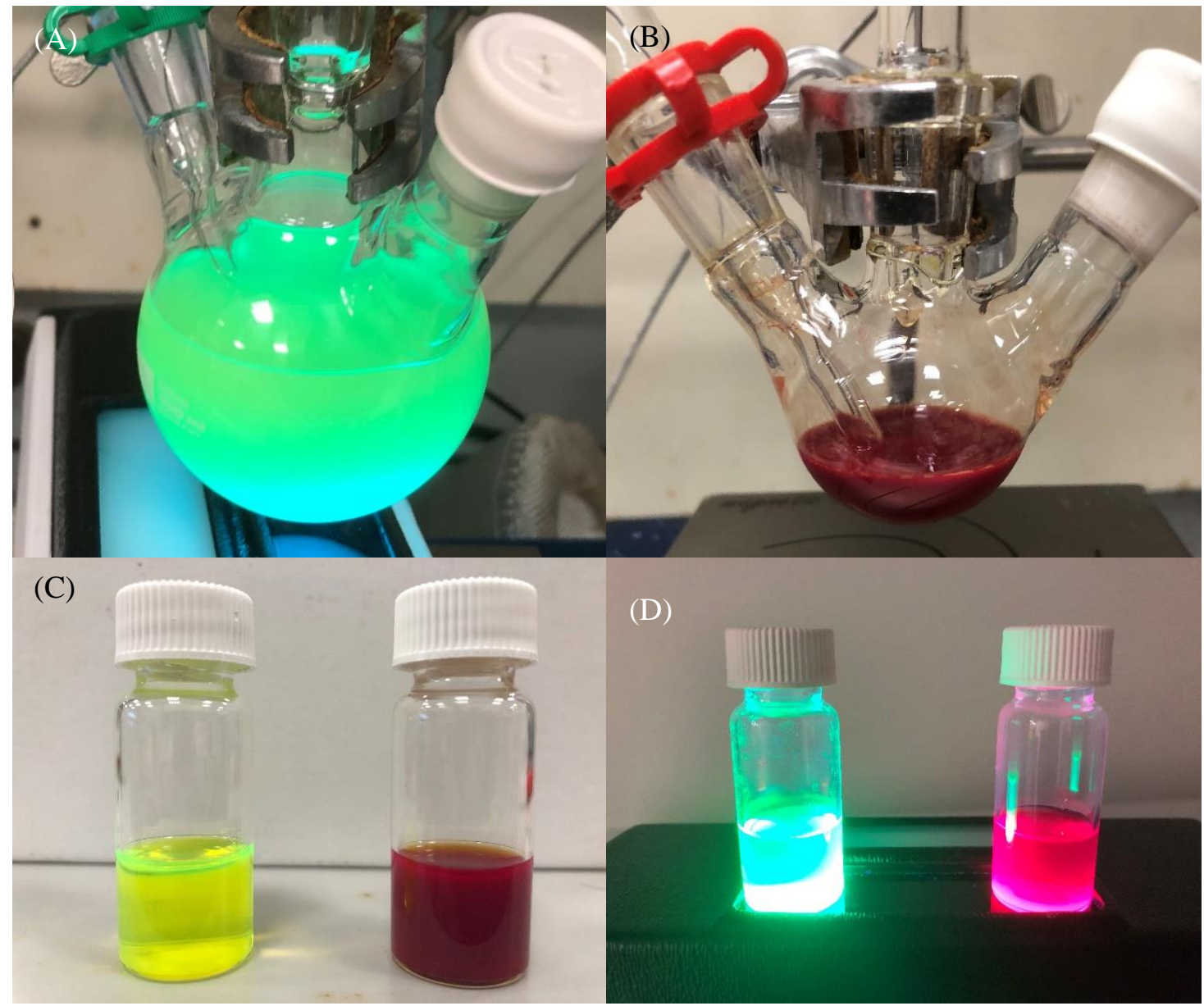

Figure 11c. (A) Reaction vessel illuminated with $U V$ containing recently synthesised $\mathrm{Cs}_{\mathrm{PbBr}} \mathrm{B}_{3}$ nanocrystals. (B) A three neck round bottom flask containing recently synthesised $\mathrm{Cs}_{\mathrm{PbI}}$ nanocrystals. (C) Hexane solutions of extracted CsPbBr ${ }_{3}-A C$ (Left) and CsPbI ${ }_{3}-A C$ (Right) nanocrystals. (D) The same solutions from (C) but fluorescing due to UV illumination.

Initial modification experiments were performed on $\mathrm{CsPbBr}_{3} . \mathrm{CsPbBr}_{3}$ nanocrystals would fail to be produced if either oleic acid $(\mathrm{OA})$ or oleylamine $(\mathrm{OAm})$ were replaced with anthracene-9-carboxylic acid (AC) or $N$-(7-aminoheptyl)anthracene-9-carboxamide (AHpAC) respectively. Adding either AC or $\mathrm{AHpAC}$ as additional reagents in an equal molar ratio to the equivalent standard ligands also resulted in reaction failure. The failure of these initial modified reactions was attributed to the incomplete dissolution of AC and AHpAC in ODE.

From these results, it was determined that the presence of significant quantities of either AC or AHpAC was detrimental to the formation of $\mathrm{CsPBr}_{3}$ nanocrystals. Further modification experiments sought to either partially substitute $\mathrm{OA}$ or OAm with either AC or AHpAC respectively, or adding the anthracene based ligands (ALs) to the standard reaction mixture, but in a significantly reduced molar equivalency to the standard ligand. 
The first of these experiments added 0.8 mmols of AHpAC (1:10 ratio of AHpAC to Oleylamine). Even the smaller quantity of $\mathrm{AHpAC}$ failed to dissolve, resulting in the formation of a fine grey precipitate within the ODE solvent. Despite the presence of this grey suspension, the injection of caesium oleate did result in the desired colour change to bright green, indicating perovskite formation.

Addition of 0.8 mmols of AHpAC to the synthesis of $\mathrm{CsPbI}_{2.25} \mathrm{Br}_{0.25}$ results in the successful formation of red fluorescent nanocrystals. However, absorption measurements of the washed particles indicated that no anthracene was present within the solution.

Addition of $0.8 \mathrm{mmol}$ of $\mathrm{AC}$ to the standard reaction synthesis of $\mathrm{CsPbr}_{3}$ also results in successful production of nanocrystals. The same is true for the synthesis of $\mathrm{CsPb}_{3}$ nanocrystals. $\mathrm{AC}$ in such a small quantity is observed to dissolve within the ODE solvent. In both cases, AC can be observed within the absorption measurements of the non-washed particles that had been extracted from the reaction mixture and resuspended in hexane. One round of precipitation and resuspension can either result in a fluorescent product that displays reduced anthracene absorption or will result in the aggregation of the nanoparticles upon resuspension. These observations suggest that the washing procedure is removing the ligands that stabilise the nanocrystals, or that the partial displacement of the standard ligands with $\mathrm{AC}$ reduces the inherent stability of the particles to the washing procedure. Therefore, data collection was performed on both washed and non-washed particles. 


\section{Absorption Measurements of Hot-Injection Synthesised $\mathrm{CsPbX}_{3}$ and $\mathrm{CsPbX}_{3}$-AL Nanocrystals}

The absorption spectra of hot-injection synthesised standard caesium lead tribromide nanocrystals $\left(\mathrm{CsPbBr}_{3} \mathrm{NCs}\right)$ displays a peak at the onset of absorption at $500 \mathrm{~nm}$. The absorption range and the correlation of this peak with the absorption onset suggest that this is an excitonic peak. Beyond this peak, towards shorter wavelengths, the intensity grows until the edge of the scanning range. One feature of note is a significant shoulder peak at $\sim 350 \mathrm{~nm}$. The cause behind this peak is unresolved, but it may be the result of contamination, certain particle sizes present within the sample, or a second exciton peak (see Figure 12d).

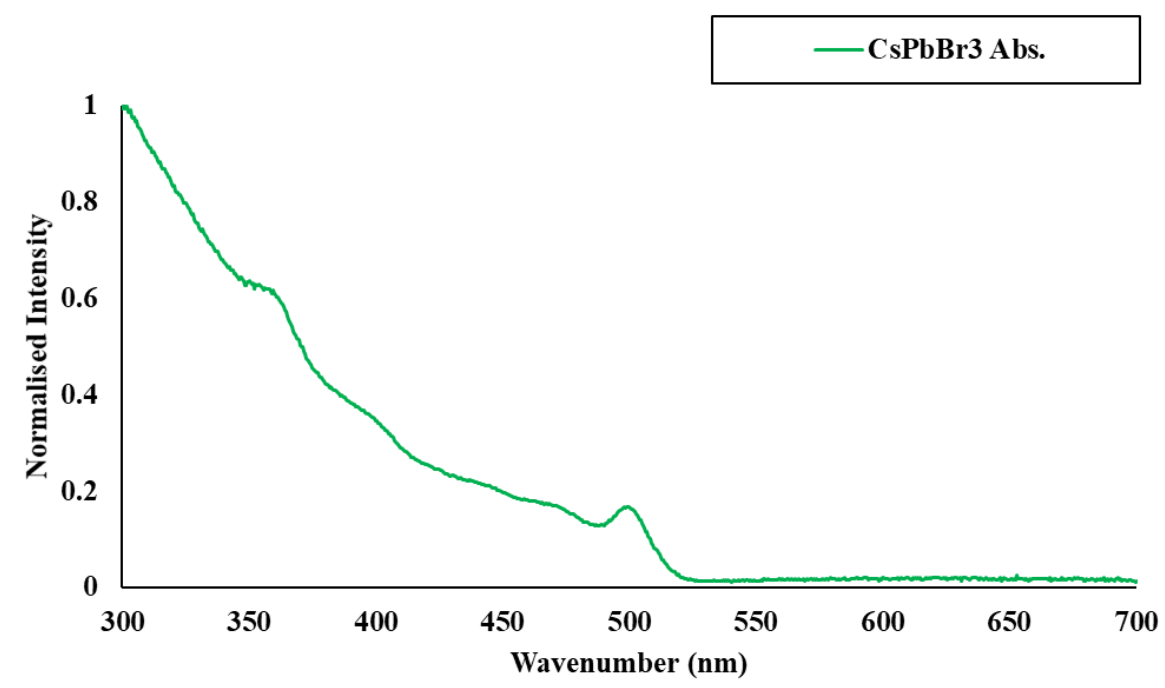

Figure 12d. Absorption spectrum of standard $\mathrm{CsPbBr}{ }_{3} \mathrm{NCs}$.

$\mathrm{CsPbBr}_{3}-\mathrm{AHpAC}$ and $\mathrm{CsPbBr}_{3}-\mathrm{AC}$ both demonstrate the same onset of absorption at $\sim 500 \mathrm{~nm}$ though the later samples lack a peak at the absorption onset as demonstrated by the standard hot-injection synthesised $\mathrm{CsPbBr}_{3} \mathrm{NCs}$. Beyond the absorption onset, all three samples demonstrate the same pattern of increasing absorption with decreasing wavelength. What separates both $\mathrm{CsPbBr}_{3}-\mathrm{ALs}$ from the standard $\mathrm{CsPbBr}_{3} \mathrm{NCs}$ are the presence of small bands of increased absorption between 400 and 350 nm (see Figure 12e.). 

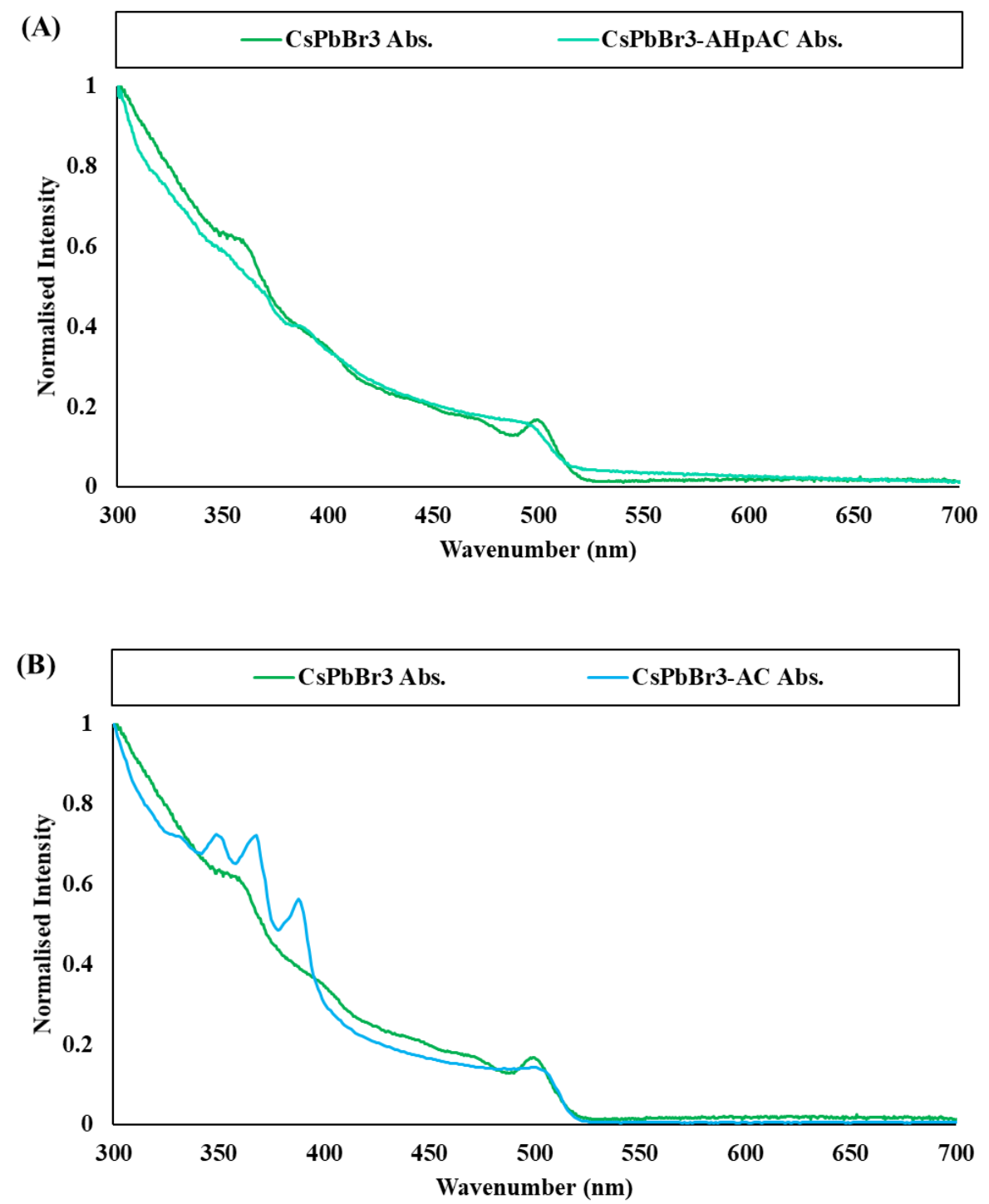

Figure 12e. Comparison between the electronic absorption of pure $A H p A C$ and $C \mathrm{PbBr} \mathrm{r}_{3}-\mathrm{AHpAC}$. (B) Comparison between the electronic absorption of pure $A C$ and $\mathrm{Cs}_{\mathrm{PbBr}}-\mathrm{AC}$.

Overlaying the absorption spectrum of pure $\mathrm{AHpAC}$ and $\mathrm{AC}$ with those of these nanocrystal samples show a correlation between the absorption peaks in the 400 to $350 \mathrm{~nm}$ range. $\mathrm{CsPbBr}_{3}-\mathrm{AC}$ demonstrates the strongest anthracene absorption, indicating a higher concentration of $\mathrm{AC}$ on the nanoparticles and therefore implying that the carboxylic acid functional group is more affective at coordinating to the particle surface than the terminal amine of AHpAC (see Figure 12f). 

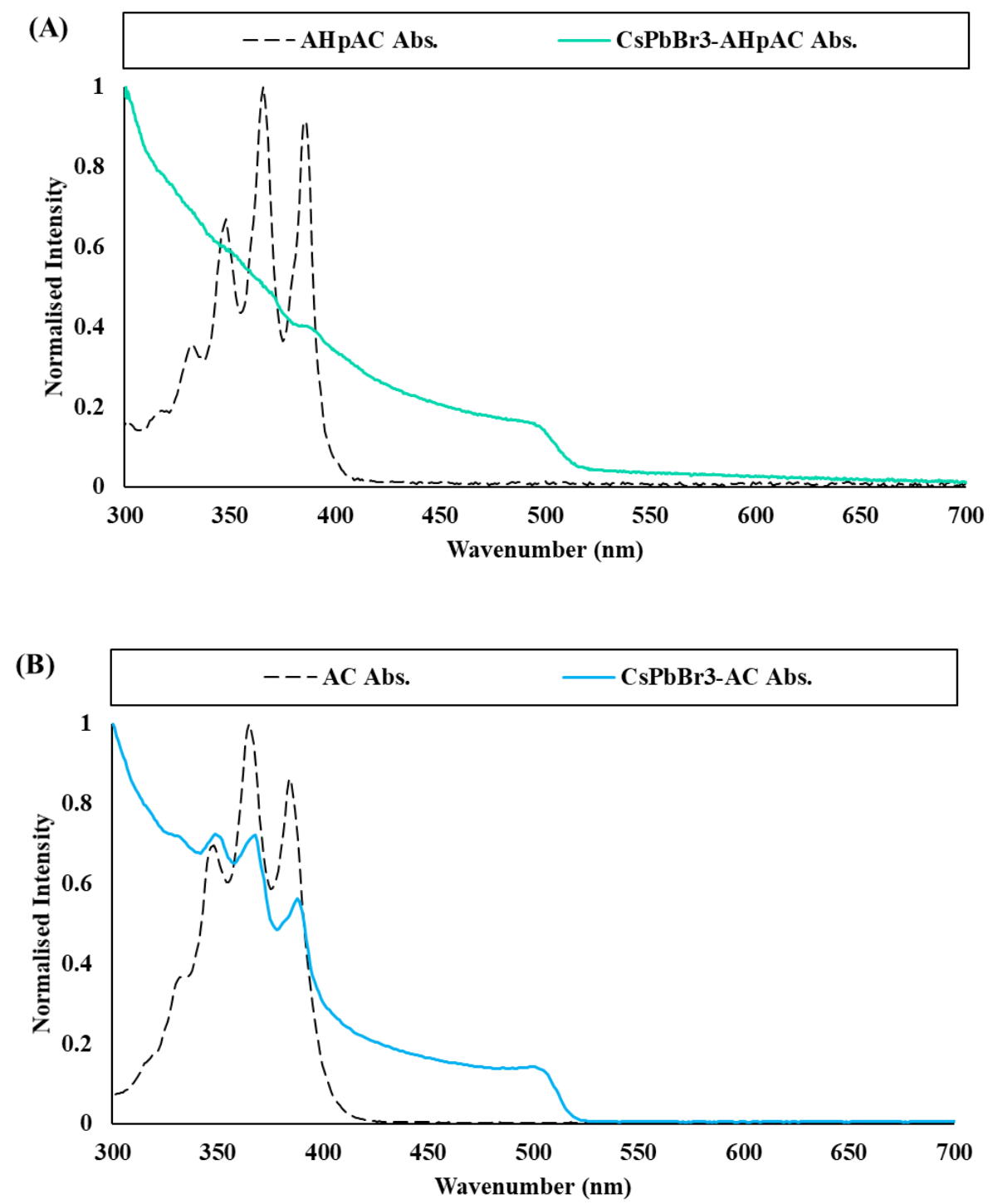

Figure 12f. (A and B) Comparison between the electronic absorption of standard CsPbBr 3 and $\mathrm{CsPbBr}_{3}-\mathrm{AHpAC}$ and $\mathrm{Cs} \mathrm{PbBr}_{3}-A C$, respectively.

The electronic absorption spectrum of $\mathrm{CsPbI}_{2.25} \mathrm{Br}_{0.75}$ shows an absorption onset of $645 \mathrm{~nm}$, well into the red region of the visible spectrum. The lack of a well-defined excitonic peak indicates a wide particle size distribution within the sample. The sample demonstrates the same trend of increasing absorption with decreasing wavelength, though this increase is non-uniform (see Figure 12g). 


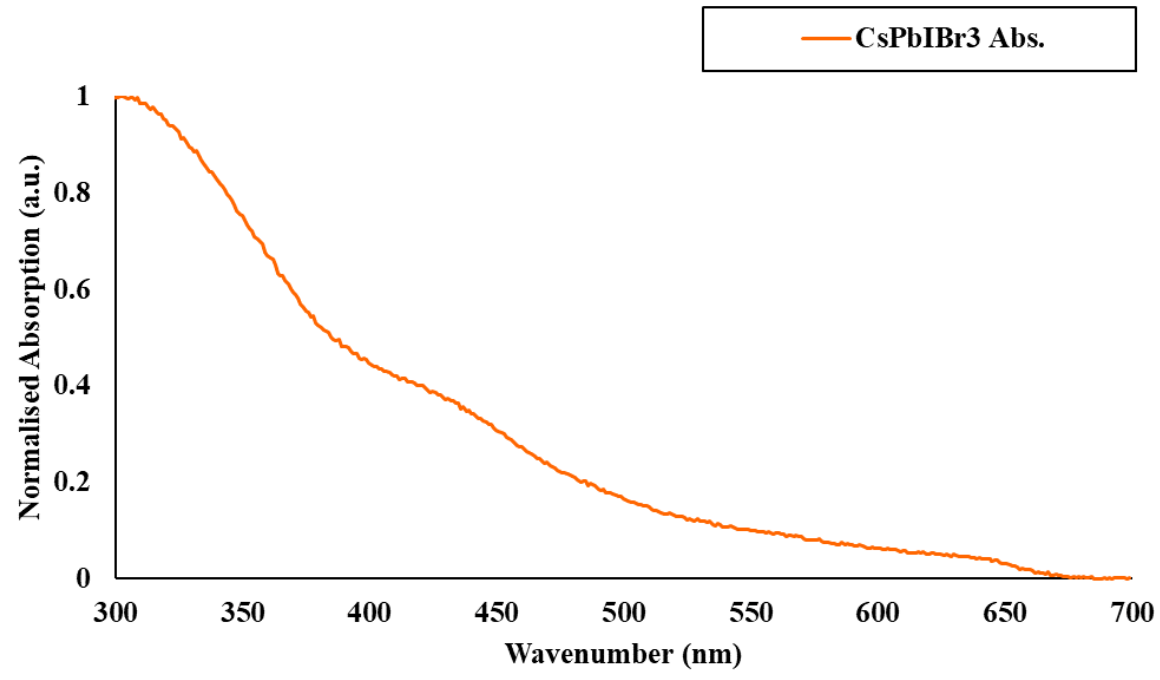

Figure 12g. Absorption spectrum of standard $\mathrm{CsPb}_{2.25}$ Br $_{0.75}$ nanocrystals.

As can be seen in Figure $\mathbf{1 2 h}$ attempts to coordinate AHpAC to $\mathrm{CsPb}_{2.25} \mathrm{Br}_{0.75}$ failed as the particles failed to demonstrate any sign of anthracene absorption. In fact, the sample almost demonstrates the exact same electronic absorption spectrum of the standard nanocrystals. The lack of anthracene suggests that the terminal amine was not sufficient to coordinate the ligand to iodine rich nanocrystals, or that the extraction procedure was sufficient to extract the ligands from the particle.

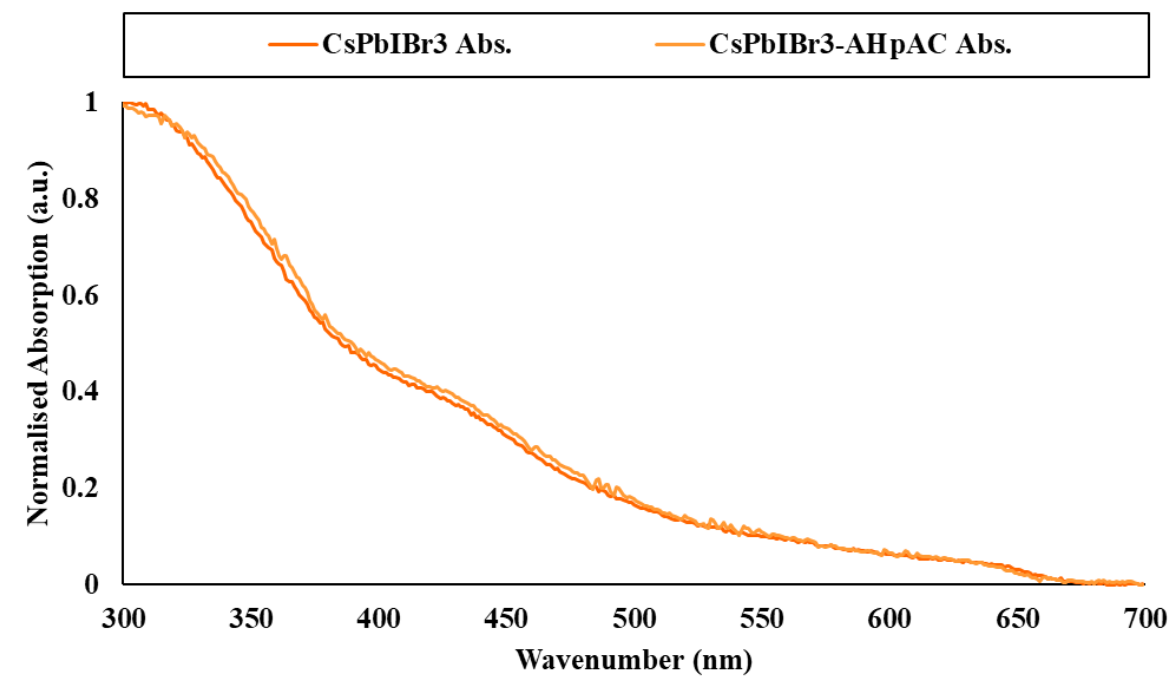

Figure 12h. Comparison between the electronic absorption spectrum of standard CsPbI $\mathrm{I}_{2.25} \mathrm{Br}_{0.75}$ NCs and CsPbI $\mathrm{I} .25 \mathrm{Br}_{0.75}-\mathrm{AHpAC} \mathrm{NCs}$

While AHpAC failed to coordinate, the addition of AC to the standard reaction mixture appeared to be successful as the absorption spectrum of crude $\mathrm{CsPbI}_{2.25} \mathrm{Br}_{0.75}$ - $\mathrm{AC}$ shows the presence of anthracene absorption (see Figure 12i.). 


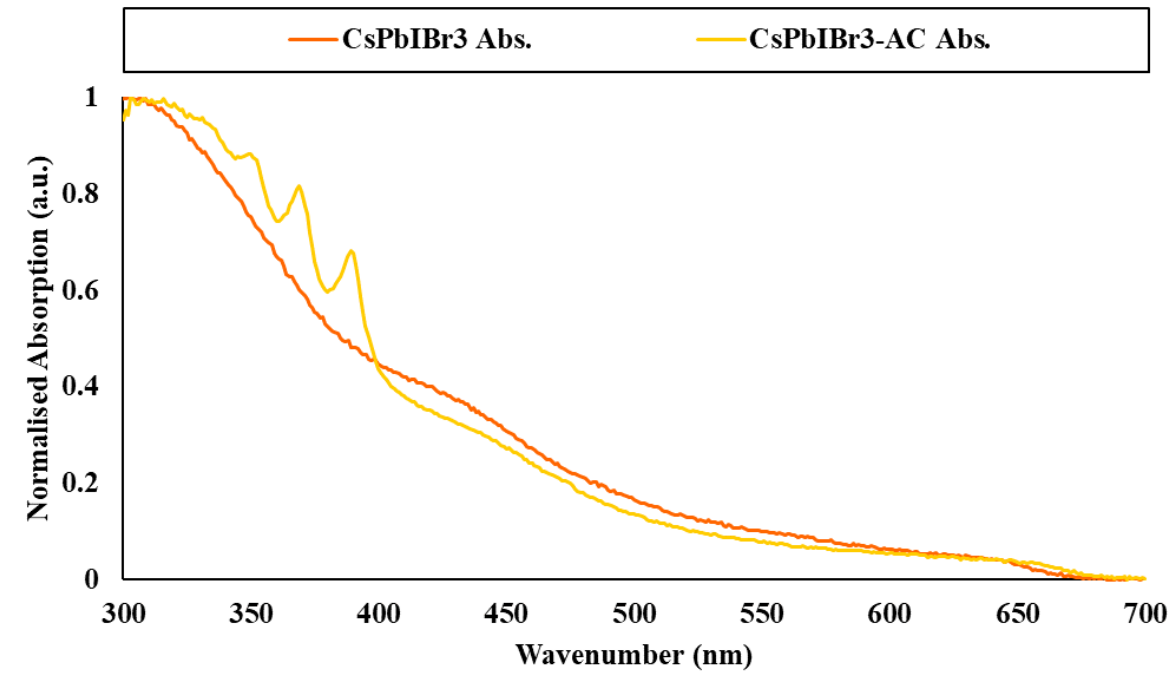

Figure 12i. Comparison between the electronic absorption spectrum of standard $\mathrm{Cs}_{\mathbf{P}} \mathrm{Pb}_{2.25} \mathrm{Br}_{0.75}$ NCs and CsPbI ${ }_{2.25} \mathrm{Br}_{0.75}-A C$ NCs respectively.

Comparing the absorption spectrum of pure $\mathrm{AC}$ with that of $\mathrm{CsPbI}_{2.25} \mathrm{Br}_{0.75}$ shows a strong correlation between the two samples absorption peaks, further confirming the presence of AC within the sample (see Figure 12j).

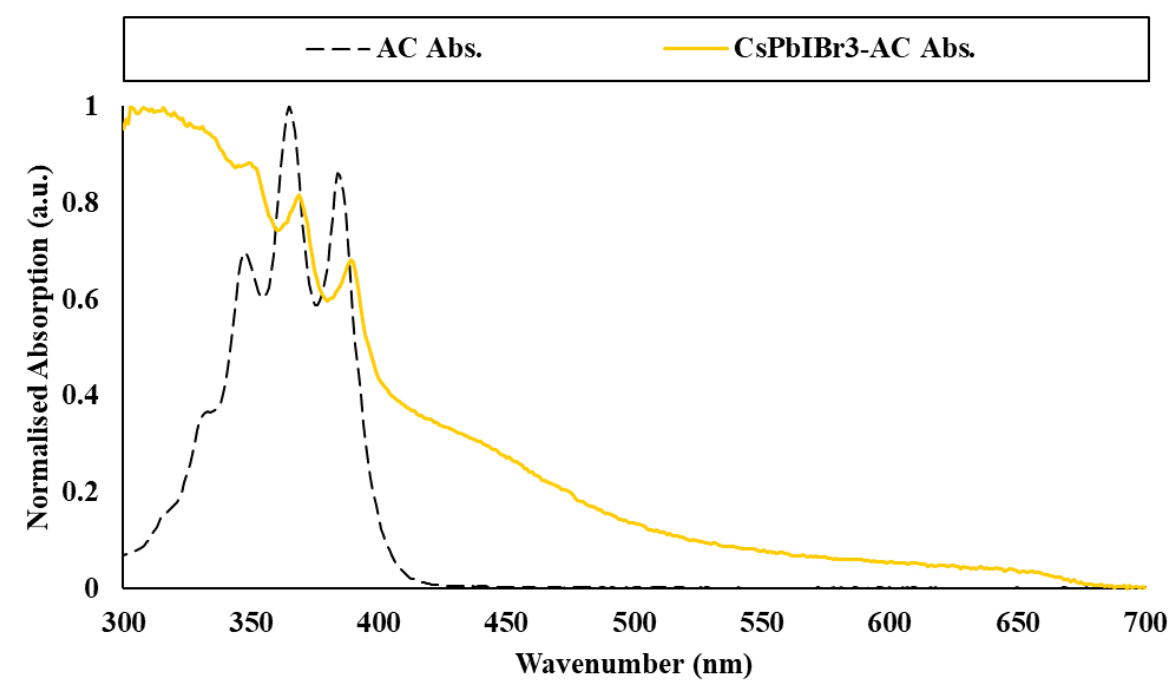

Figure 12j. Comparison between the electronic absorption spectrum of pure AC and $\mathrm{Cs}_{\mathrm{PbI}} \mathrm{2.25}_{\mathrm{Br}} \mathrm{Br}_{0.75}$-AC NCs. Green highlight indicates positive peak correlation.

Based upon the results of $\mathrm{CsPb}_{2.25} \mathrm{Br}_{0.75}$, further efforts to couple anthracene ligands to red emission perovskite nanocrystals focused on AC only. Once the replicable synthesis of bromine free $\mathrm{CsPb}_{3}$ was achieved further experimentation and data collection for red emitting caesium lead halide perovskites focused on $\mathrm{CsPb}_{3}$ nanocrystals. The absorption spectrum of standard $\mathrm{CsPbI}_{3} \mathrm{NC}^{\prime}$ 's is highly reminiscent 
of $\mathrm{CsPbI}_{2.25} \mathrm{Br}_{0.75}$ (see Figure 12k). Despite the absence of bromine, the absorption onset has not redshifted as expected, indicating that the small bromine impurity does not significantly influence the band gap of the overall material. The sample also demonstrates the non-uniform increase in absorption intensity, showing that this is a common feature of iodine rich caesium lead trihalide perovskites.

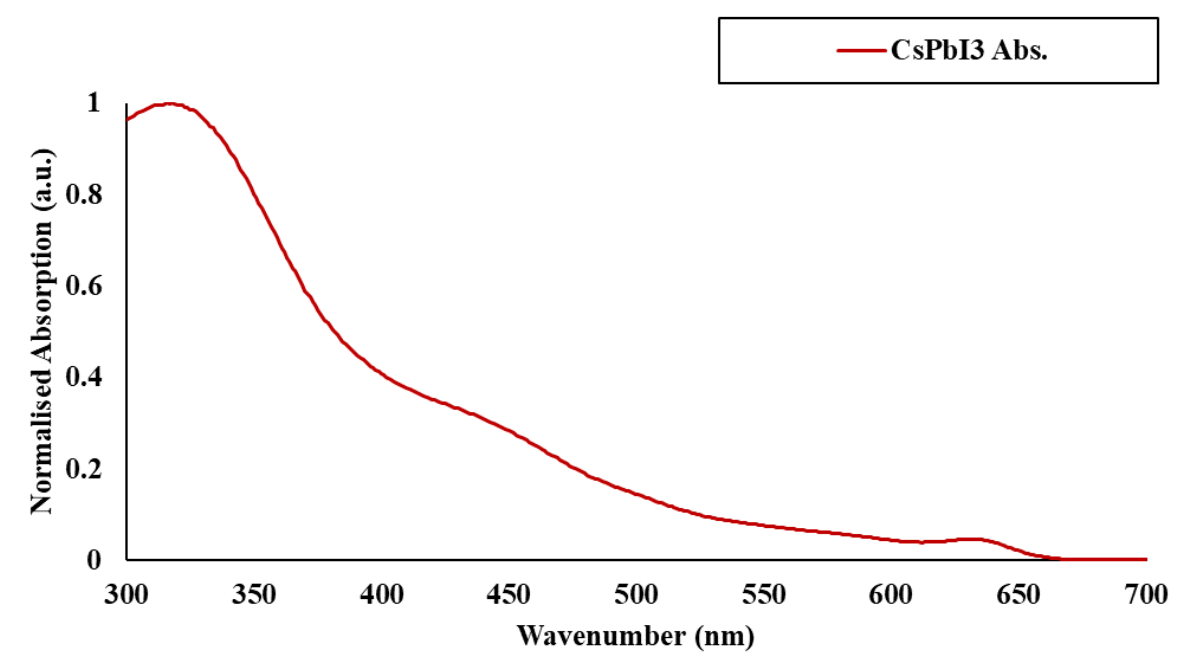

Figure 12k. Absorption spectrum of standard $\mathrm{CsPbI}_{3}$ nanocrystals.

Like their bromine counter parts, $\mathrm{CsPb}_{3}$ nanocrystals that were formed in the presence of $0.8 \mathrm{mmol}$ of $\mathrm{AC}$ show the presence of anthracene absorption peaks, demonstrating that the versatility of AC across a series of perovskites (see Figure 12l). Though these peaks are less prominent than in the previously discussed samples, high concentrations of anthracene have proven to be detrimental to perovskite performance and therefore a low concertation may prove to be more beneficial. Once again the anthracene peaks correlate well with those of pure AC (see Figure 12m).

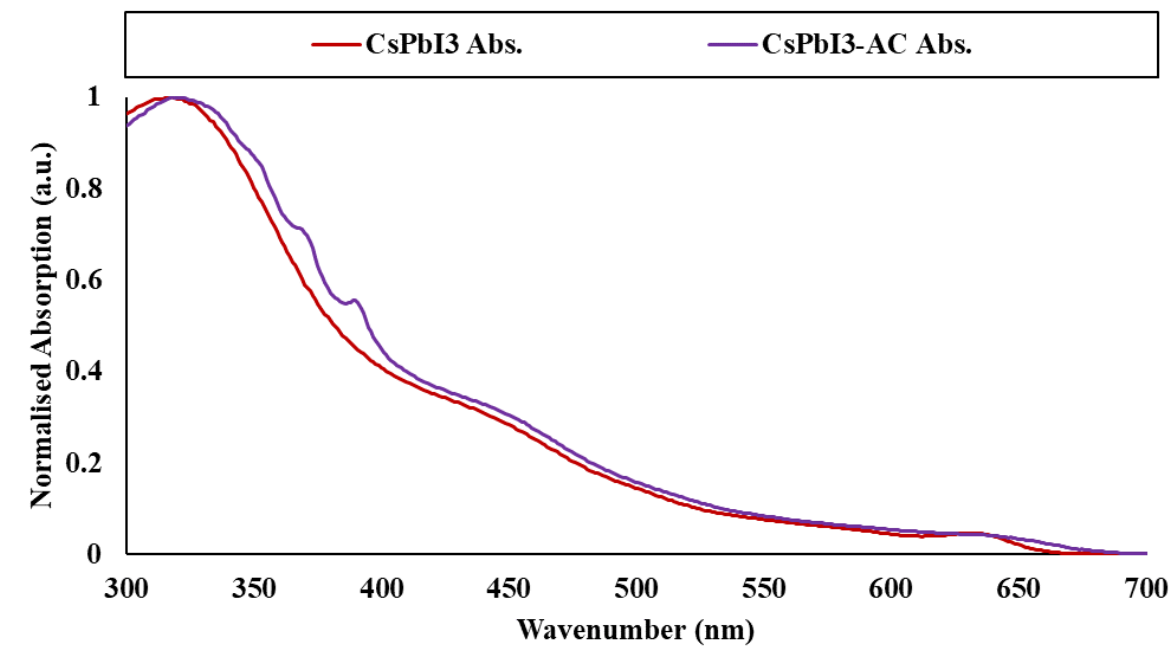

Figure 12l. (A) Comparison between the absorption spectra of standard $\mathrm{CsPbI}_{3} \mathrm{NCs}$ and $\mathrm{CsPbI} 3^{-}$ AC NCs. (B) Comparison between the absorption spectra of pure AC and CsPbI ${ }_{3}-A C N C s$. 


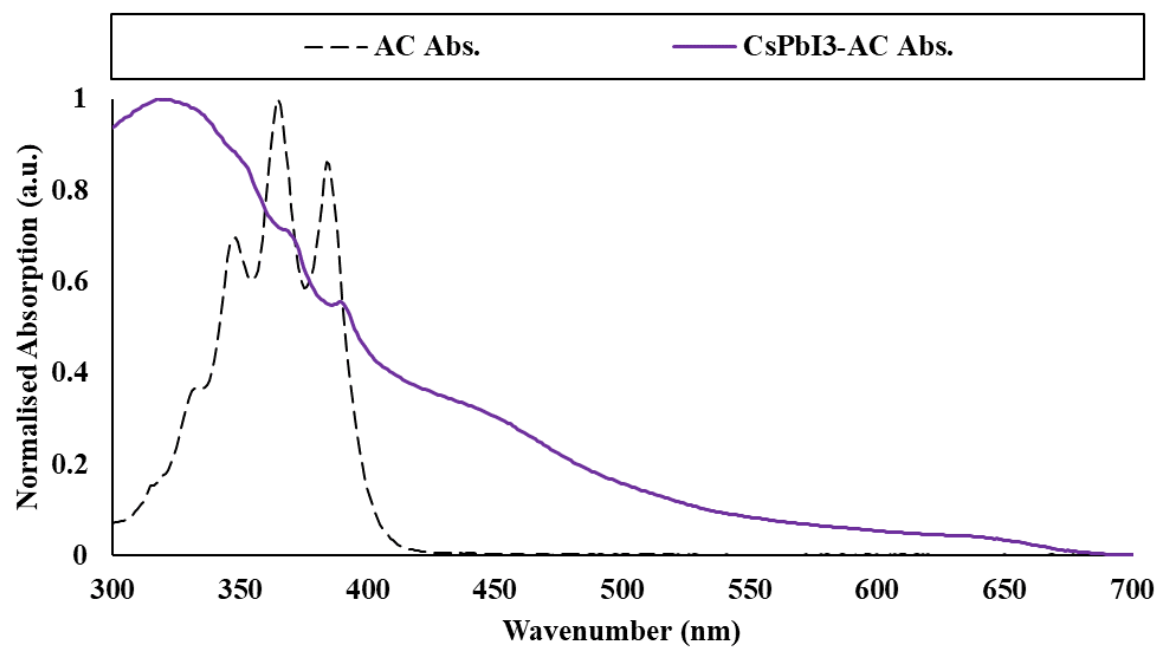

Figure 12m. (A) Comparison between the absorption spectra of standard $\mathrm{CsPbI}_{3} \mathrm{NCs}$ and $\mathrm{CsPbI}_{3}-\mathrm{AC} \mathrm{NCs}$. (B) Comparison between the absorption spectra of pure $\mathrm{AC}$ and $\mathrm{Cs} \mathrm{PbI}_{3}-\mathrm{AC}$ NCs.

As stated in the observations, the washing procedure proved to be detrimental to the presence of AL in all samples as absorption measurements taken after washing and redispersion showed reduced anthracene absorption in comparison with the previous measurements of the crude samples (see Figure $12 n$ and 12o). There are several possible explanations for this; firstly, it is possible that the AL was never coordinated to the nanocrystals. The nanocrystals would have been contaminated with AL containing reaction mixture during the extraction process and would have dispersed with the nanoparticles in the hexane solvent. Such a hypothesis can account for their presence within the initial absorption spectra while the washing procedure would have partially reduced this contamination.

Another possibility is that the AC ligand is attached, but that the washing procedure has partially degraded the nanoparticles and removed the AC ligands. It is widely known that for perovskites, the stabilising ligands are liable, allowing for the development of an equilibrium between coordinated ligands and non-coordinated ligands in solution. ${ }^{73}$ Dispersal of washed nanocrystals into a clean solvent draws the coordinated ligands away from the nanoparticles, facilitating aggregation and degradation. The same phenomenon could easily explain the removal of the ALs as they are reasonably soluble in mixtures of butanol with either toluene, hexane, or ODE, mixtures that the nanoparticles were exposed to during the extraction and washing procedures. 

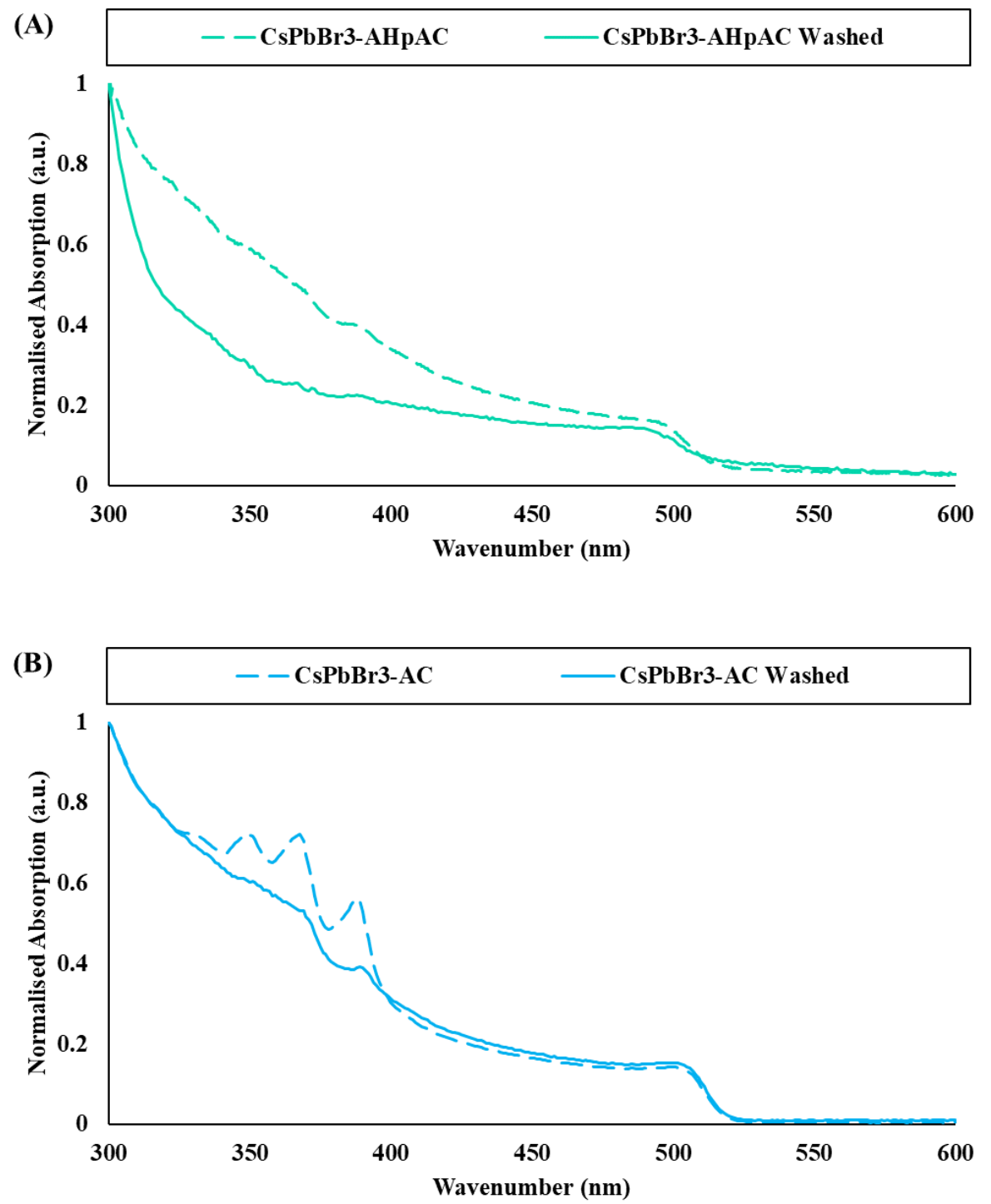

Figure 12n. Comparisons between the absorption spectra of $\mathrm{CsPBBr}_{3}-A L s$ before and after wash, illustrating the detrimental effect that the washing procedure can have on the presence of ALs. 

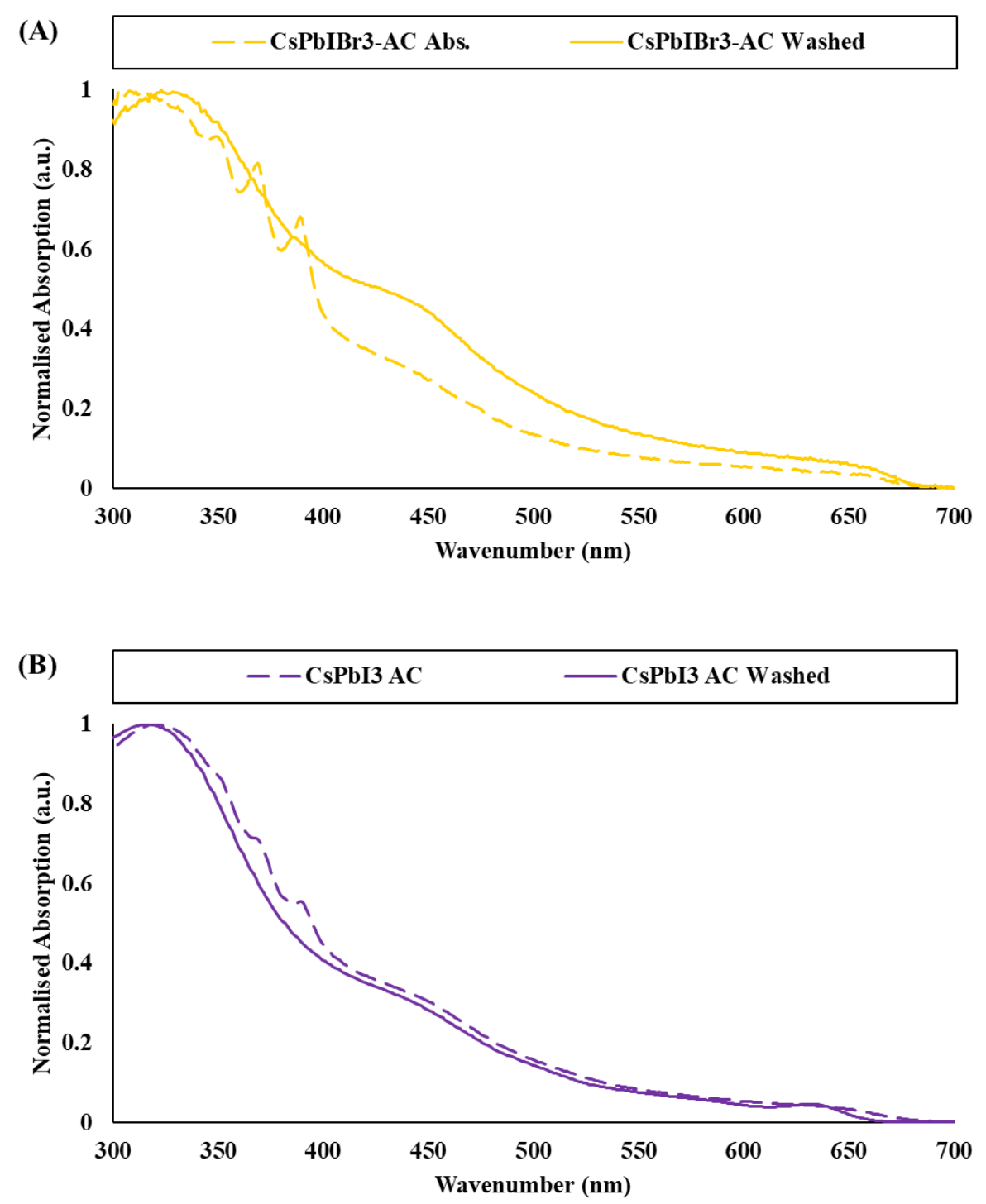

Figure 120. Comparisons between the absorption spectra of $\mathrm{CsPbI}_{2.25} \mathrm{Br}_{0.75}-\mathrm{AC}$ and $\mathrm{CsPbI}_{3}-\mathrm{AC}$ before and after wash, illustrating the detrimental effect that the washing procedure can have on the presence of $A C$.

Both explanations can explain the electronic absorption data and therefore such data on its own cannot provide conclusive evidence in support of either theory. Due to the detrimental influence of the washing procedure, certain samples were not washed as otherwise data could not be collected. 


\section{Photoluminescence of Hot-Injection Synthesised $\mathrm{CsPbX}_{3}$ and $\mathrm{CsPbX}_{3}$-AL Nanocrystals}

The electronic emission spectra of $\mathrm{CsPbr}_{3}$ comprises of one emissive peak at $507 \mathrm{~nm}$. The peak is relatively sharp, indicating a relatively narrow range of particle sizes. The two $\mathrm{CsPBBr}_{3}-\mathrm{AL} \mathrm{NCs}$ show strong correlation with that of the standard $\mathrm{CsPbBr}_{3} \mathrm{NCs}$ (see Figure 12p). For $\mathrm{CsPbBr}_{3}-\mathrm{AHpAC}$, the peak emission wavelength is blue shifted with respect to that of the standard materials peaks emission, while $\mathrm{CsPbr}_{3}-\mathrm{AC}$ displays an identical peak wavelength. The blueshift in the peak emission wavelength for $\mathrm{CsPbBr}_{3}-\mathrm{AHpAC}$ is likely to be a result of the temperature of the reaction mixture during the hot-injection procedure as opposed to the presence of AHpAC.
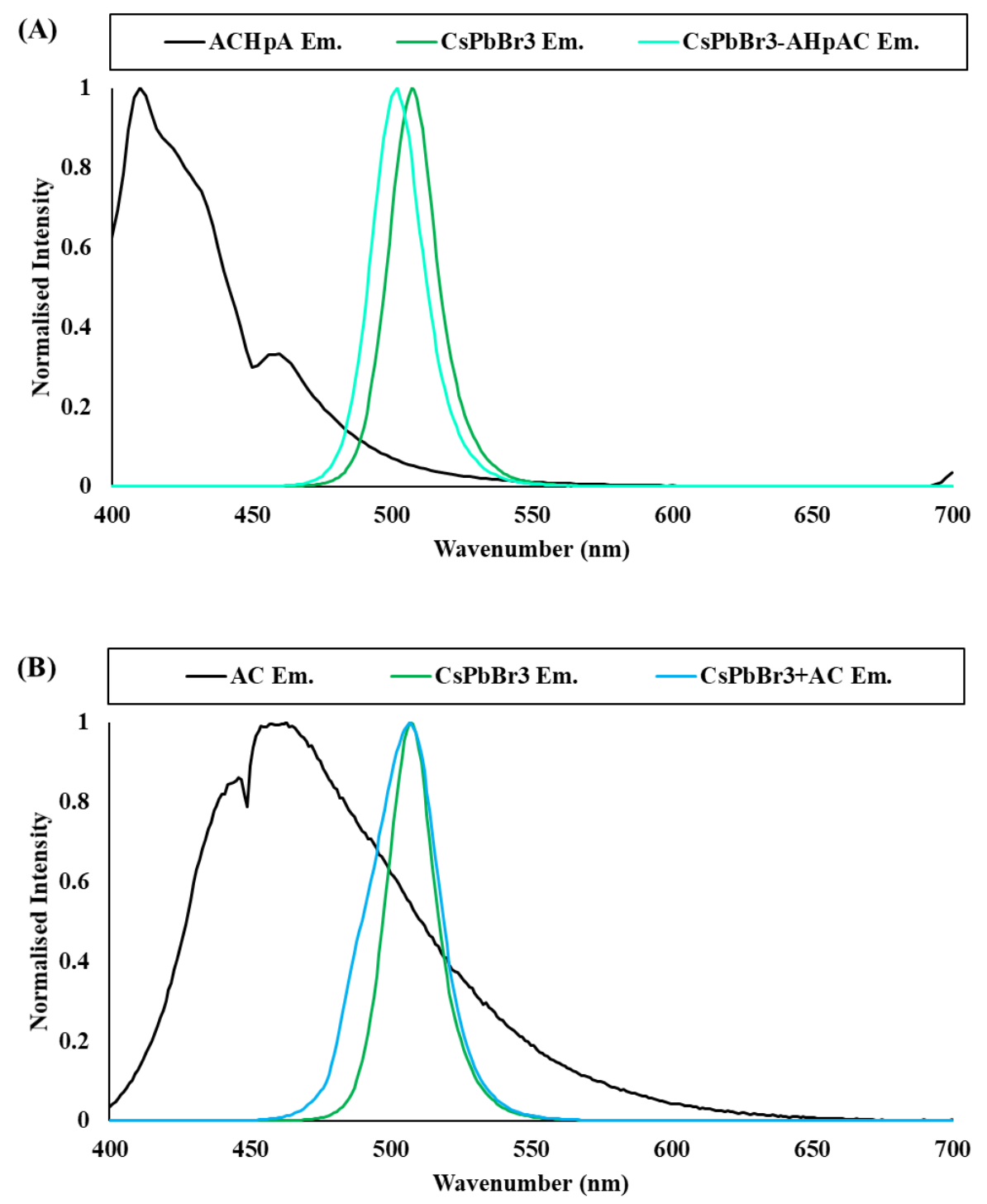

Figure 12p. Comparison between the electronic emission spectra of pure $\mathrm{AHpAC}, \mathrm{CsPbBr} 3 \mathrm{NCs}$, and $\mathrm{CsPbBr}_{3}-\mathrm{AHpAC} \mathrm{NCs.} \mathrm{(B)} \mathrm{Comparison} \mathrm{between} \mathrm{the} \mathrm{electronic} \mathrm{emission} \mathrm{spectra} \mathrm{of} \mathrm{pure} \mathrm{AC,}$ $\mathrm{Cs}_{\mathrm{PbBr}} \mathrm{NCs}$, and $\mathrm{Cs}_{\mathrm{PbBr}}-\mathrm{AC} \mathrm{NCs}$. 
Neither of the two samples demonstrate any significant anthracene emission. This may be the result of the nanoparticles strongly absorbing any emission from the anthracene or a non-radiative energy transfer process that is quenching anthracene emission, or a combination of both processes.

$\mathrm{CsPbI}_{3}-\mathrm{NCs}$ display a single peak at $647 \mathrm{~nm}$. The peak is quite broad, indicating a wide range of particles sizes. $\mathrm{CsPbI}_{3}-\mathrm{AC}$ also displays a single peak; however, the peak emission wavelength is 665 $\mathrm{nm}$ which is $18 \mathrm{~nm}$ redshifted with respect to the standard $\mathrm{CsPbI}_{3} \mathrm{NCs}$, meaning that there is a larger average particle size within the sample. The emission peak is broadened in comparison with that of the standard nanoparticles, an indication of an even greater range of particle sizes for this sample. Like the two $\mathrm{CsPbBr}_{3}-\mathrm{AL}$ samples, there is no anthracene emission and therefore indicative of reabsorption by the nanoparticles or an energy transfer process that is quenching anthracene emission (see Figure 12q).

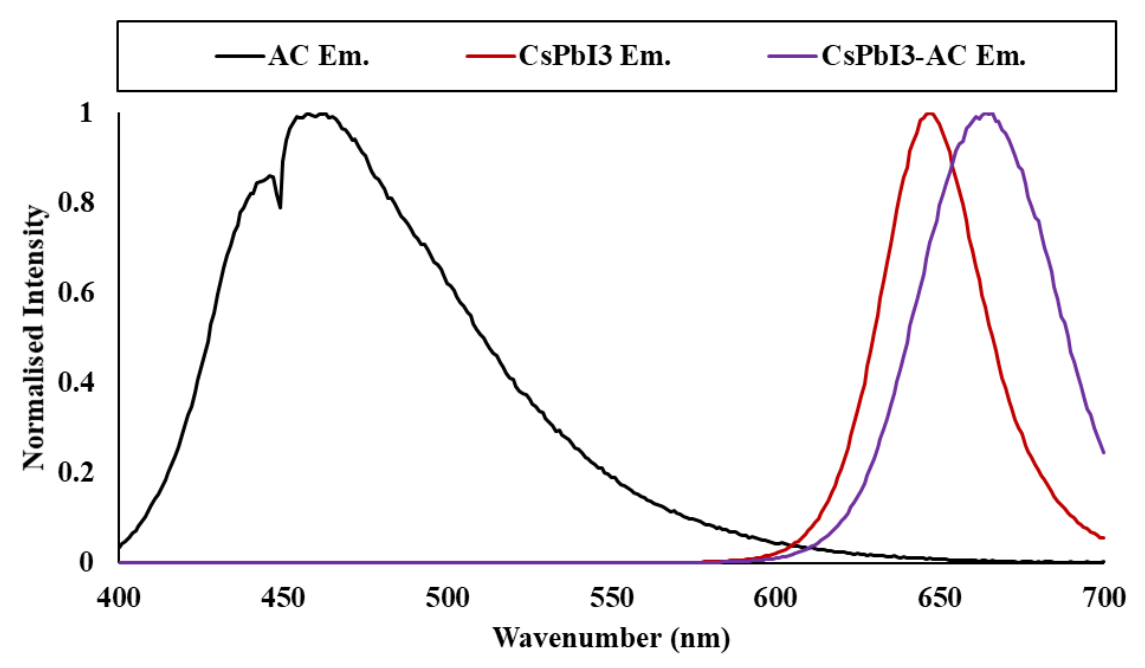

Figure 12q. Comparison between the electronic emission spectra of pure $\mathrm{AC}, \mathrm{CsPbI} \mathrm{H}_{3} \mathrm{NCs}$, and $\mathrm{CsPbI}_{3}-\mathrm{AC} \mathrm{NCs}$.

In the case of a non-radiative energy transfer process, such a phenomenon can arise from two different scenarios. One, the AL ligands are coordinated to the nanocrystals and the fixed distance between the two chemical species allows for the effective transfer of energy between the two species. Alternatively, the AL is non-coordinated, but the relative concentration of the solution allows for the two species to regularly come into close enough proximity to allow for non-radiative energy transfer and therefore quenching of AL fluorescence. Therefore, these measurements cannot prove that the AL ligands are bound to the perovskite nanocrystals. However, energy transfer between coordinated $\mathrm{AC}$ and $\mathrm{CsPbX}$ will be more effective due to the fixed distance between the acceptor and donor and therefore is more likely at explaining the complete lack of anthracene emission that is seen in these three samples. 


\section{Electronic Excitation of Hot-Injection Synthesised $\mathrm{CsPbX}_{3}$ and $\mathrm{CsPbX}_{3}-\mathrm{AL}$ Nanocrystals}

The electronic excitation spectrum of standard $\mathrm{CsPbBr}_{3} \mathrm{NCs}$ appears as expected. As with absorption, the intensity of excitation increases with decrease wavelength, though, beyond $330 \mathrm{~nm}$, the intensity drops again, likely due to a high concentration of NCs preventing these highly absorbed wavelengths from exciting the bulk of the sample (see Figure 12r).

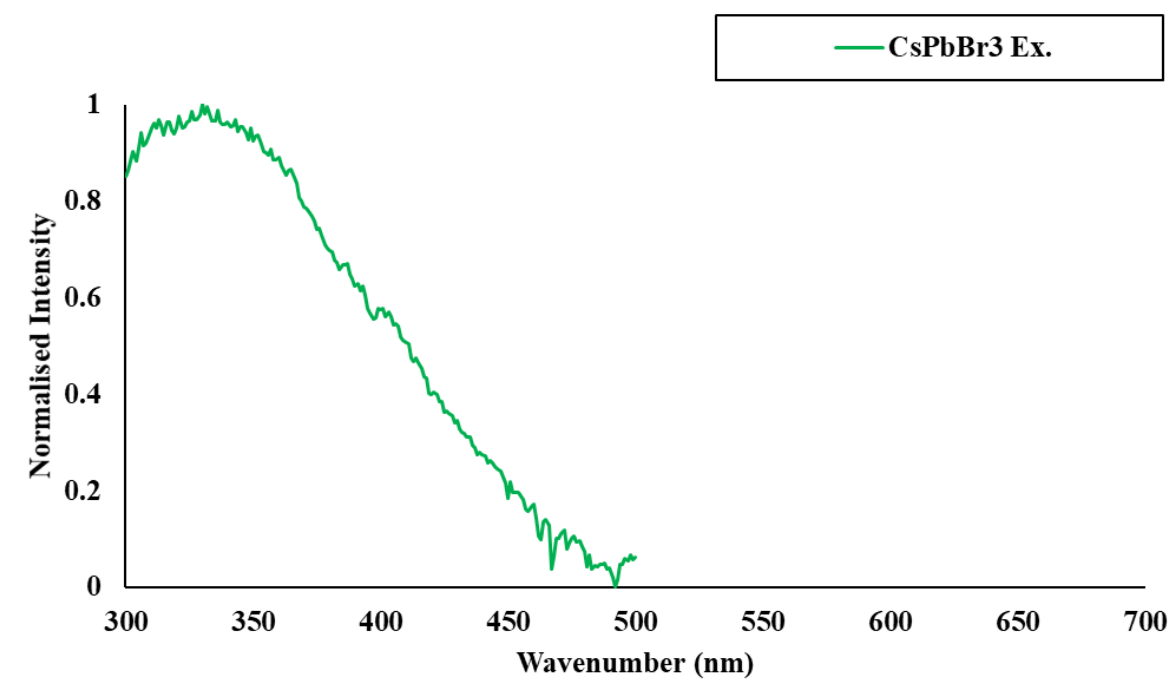

Figure 12r. Excitation spectrum of standard $\mathrm{CsPbBr}, \mathrm{NCs}$.

Both $\mathrm{CsPbBr}_{3}-\mathrm{AHpAC}$ and $\mathrm{CsPbBr}_{3}-\mathrm{AC}$ display electronic excitation spectra that closely matches that of the standard nanoparticles in terms of shape, though both samples lack the decrease in intensity between 350 and $300 \mathrm{~nm}$ (see Figure 12s and 12t).

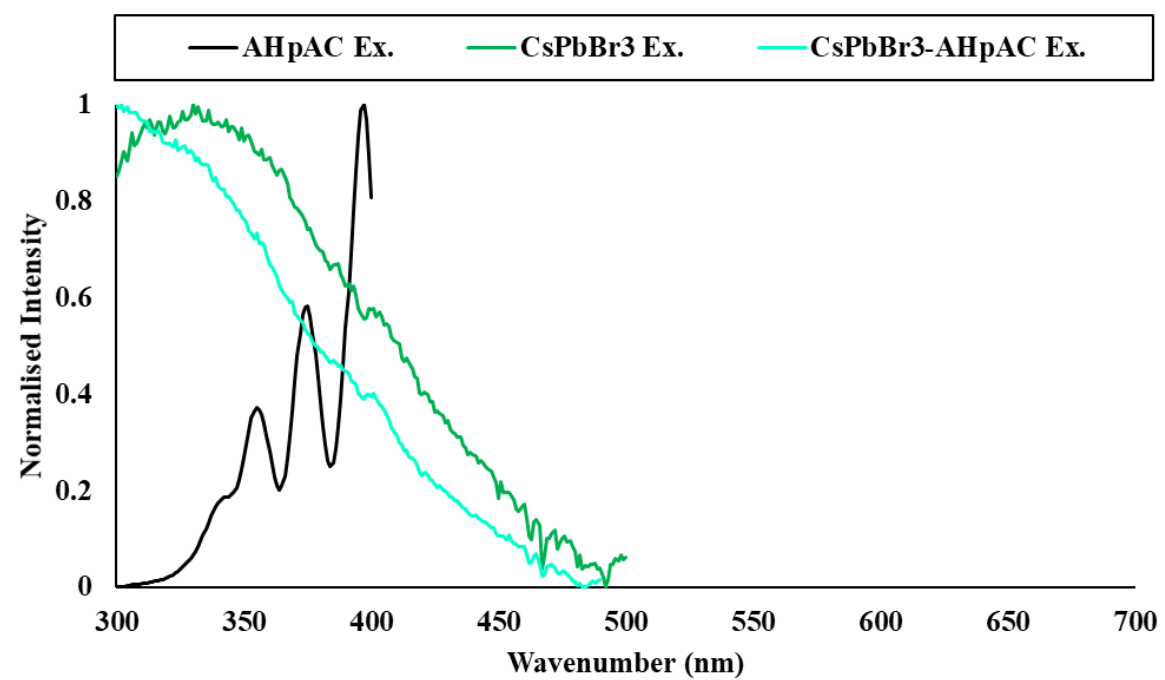

Figure 12s. Comparison between the electronic excitation spectra of pure $\mathrm{AHpAC,} \mathrm{CsPbBr}_{3}$ NCs, and CsPbBr $-A H p A C N C s$. 


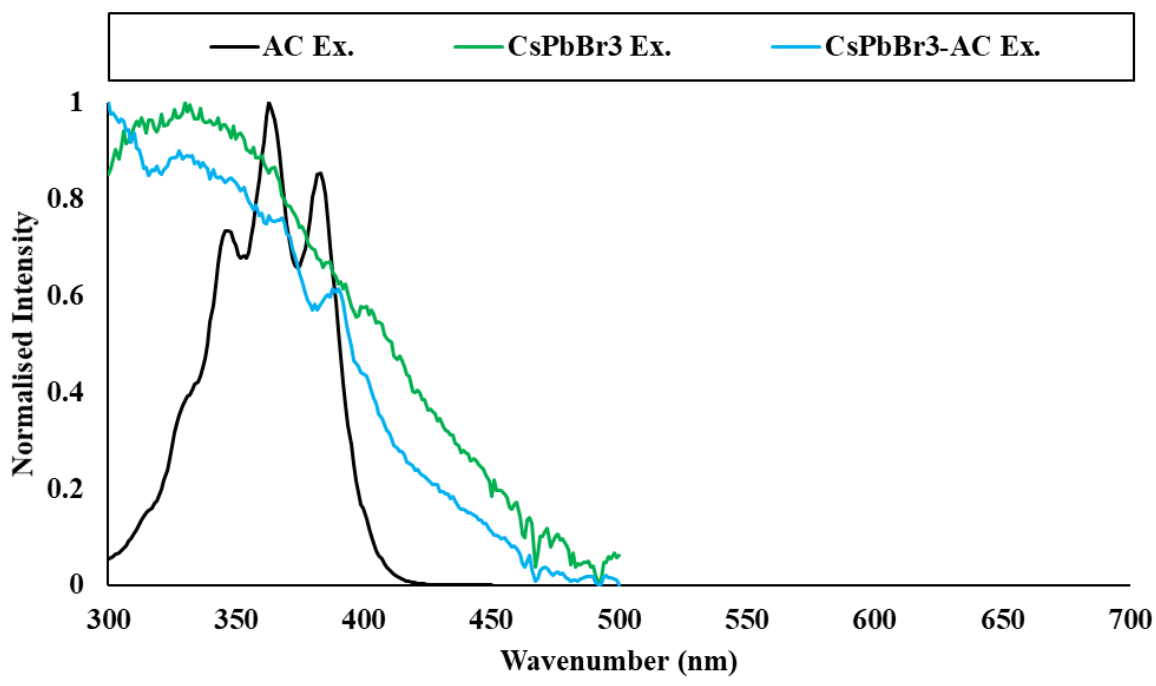

Figure 12t. Comparison between the electronic excitation spectra of pure $\mathrm{AC}, \mathrm{CsPbBr}_{3} \mathrm{NCs}$, and $\mathrm{CsPBBr}_{3}-\mathrm{AC} \mathrm{NCs.}$

$\mathrm{CsPbBr} 3-\mathrm{AC}$ also demonstrates a heightened excitation intensity between $\sim 400$ and $300 \mathrm{~nm}$, with three highly noticeable peaks. These peaks correlate well with the excitation spectrum of pure AC, indicating that anthracene excitation is contributing to 507 emission. However, the emission spectrum of both AC and AHpAC shows that both molecules emit significantly in 500 to $550 \mathrm{~nm}$ range. However, both NC samples lacked any significant signs of anthracene emission, indicating a possible energy transfer process. $\mathrm{CsPbBr}_{3}-\mathrm{AHpAC}$ also demonstrates a weak shoulder peak at $\sim 400 \mathrm{~nm}$. If this is a sign of anthracene, it would suggest that the increased separation of the anthracene functional group to the NC significantly reduces the efficiency of energy transfer, or that there is a very small amount of AHpAC within the sample.

The intensity of the 300 to $400 \mathrm{~nm}$ excitation peaks displayed by $\mathrm{CsPbBr}_{3}-\mathrm{AC} \mathrm{NCs}$ allows for an effective comparison with the excitation spectrum of pure AC. Such a comparison shows a significant displacement of the peaks towards longer wavelengths. This shift is significant enough to suggest a negative correlation. Such a correlation would mean that excitation of the ligand is preventing $507 \mathrm{~nm}$ emission. However, comparison with the absorption spectrum of $\mathrm{CsPbr}_{3}-\mathrm{AC}$ NCs shows a positive correlation, meaning that peak anthracene absorption is indeed correlated with heightened excitation and subsequently perovskite emission (see Figure 12u). Therefore, the shift in excitation peaks is likely to be a result of the coordination of the AC ligand to the nanoparticles. 


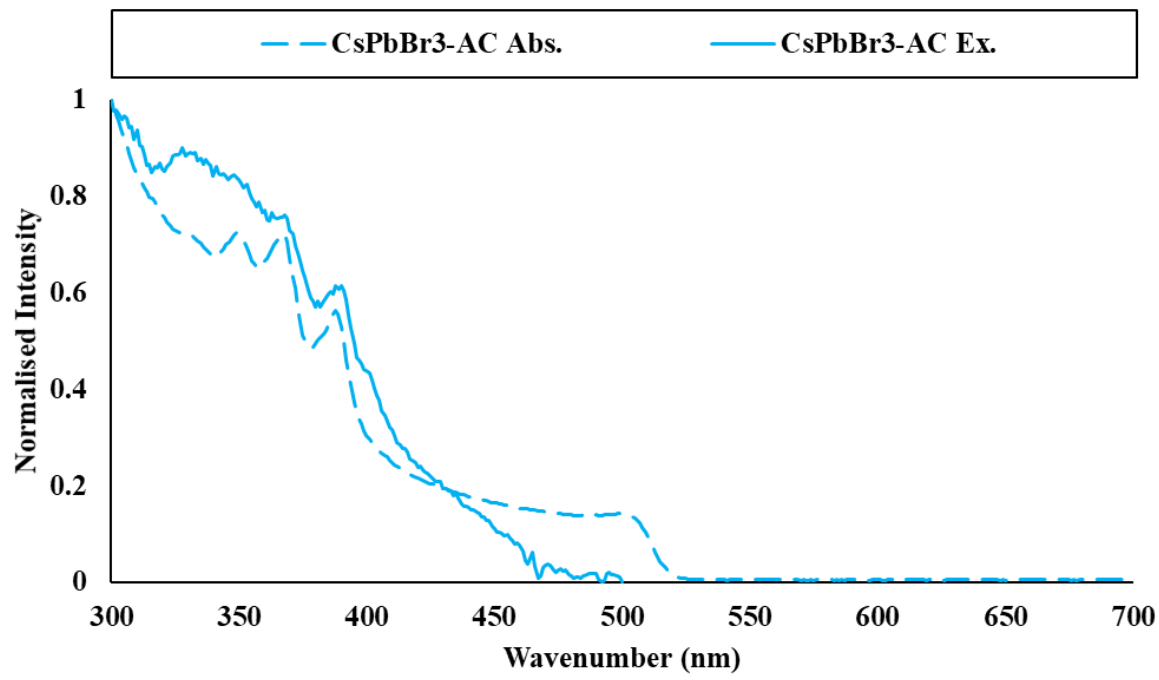

Figure 12u. Comparison between the electronic excitation spectrum of $\mathrm{CsPBBr}_{3}-\mathrm{AC}$ and the absorption spectrum of $\mathrm{Cs} \mathrm{PbBr}_{3}-\mathrm{AC}$.

The electronic excitation spectrum of $\mathrm{CsPbI}_{3}-\mathrm{AC}$ almost perfectly matches that of standard $\mathrm{CsPbI}_{3}$ except for two peaks at 365 and $389 \mathrm{~nm}$ (see Figure 12v.).

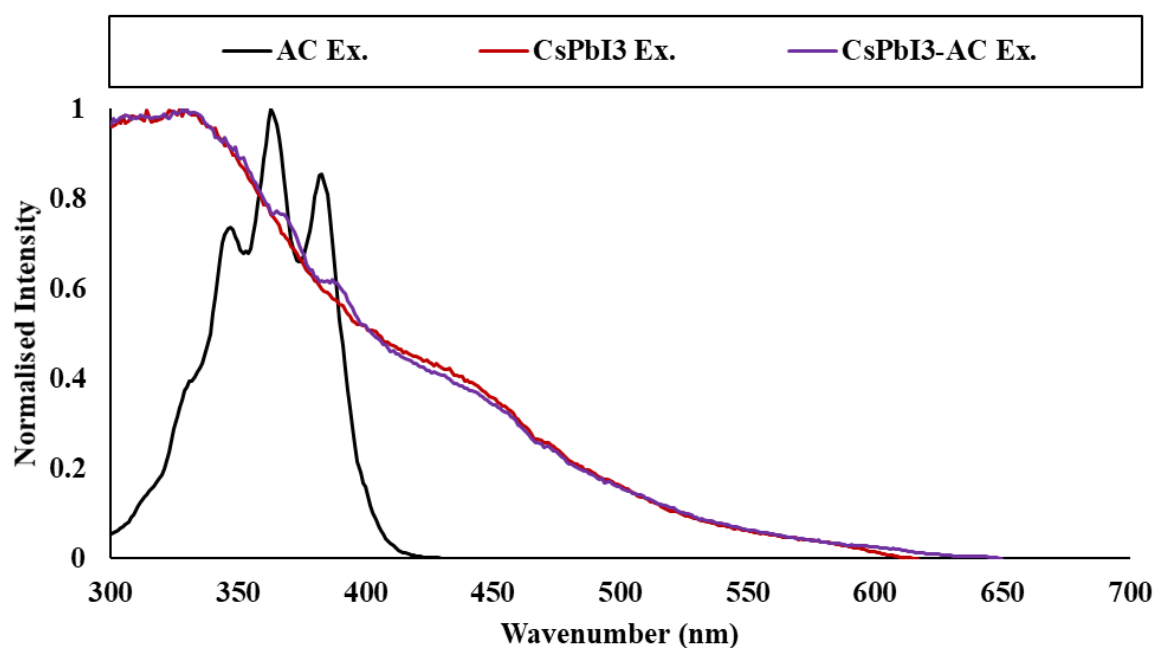

Figure 12v. Comparison between the electronic excitation spectra of pure $\mathrm{AC}, \mathrm{Cs} \mathrm{PbI}_{3} \mathrm{NCs}$, and CsPbI ${ }_{3}-A C N C s$.

Unlike the bromide samples, the emission of $\mathrm{CsPbI}_{3}-\mathrm{AC}$ hardly overlaps with that of $\mathrm{AC}$ (refer back to Figure 12q). This fact coupled together with the lack of any significant anthracene emission at 410 to $450 \mathrm{~nm}$ is strongly suggestive of a non-radiative energy transfer between the two species.

Like in the sample $\mathrm{CsPBr}_{3}-\mathrm{AC}$, overlaying the excitation spectra of this sample with that of pure $\mathrm{AC}$ shows that the peaks of these two sample do not match, while the absorption spectrum of $\mathrm{CsPb}_{3}-\mathrm{AC}$ 
positively correlates (see Figure 12w.). Again, the electronic absorption and excitation spectral signals of anthracene have redshifted in the presence a $\mathrm{CsPbX}_{3}$ nanocrystals, suggestive of coordination between the two reagents.

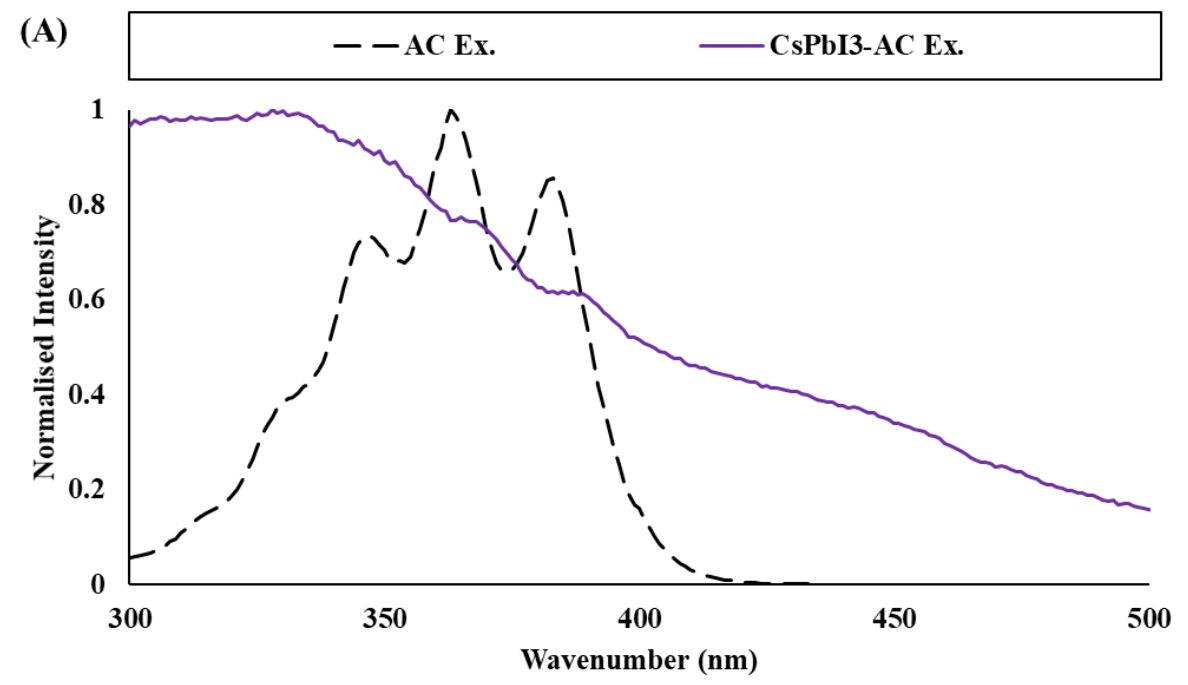

(B)
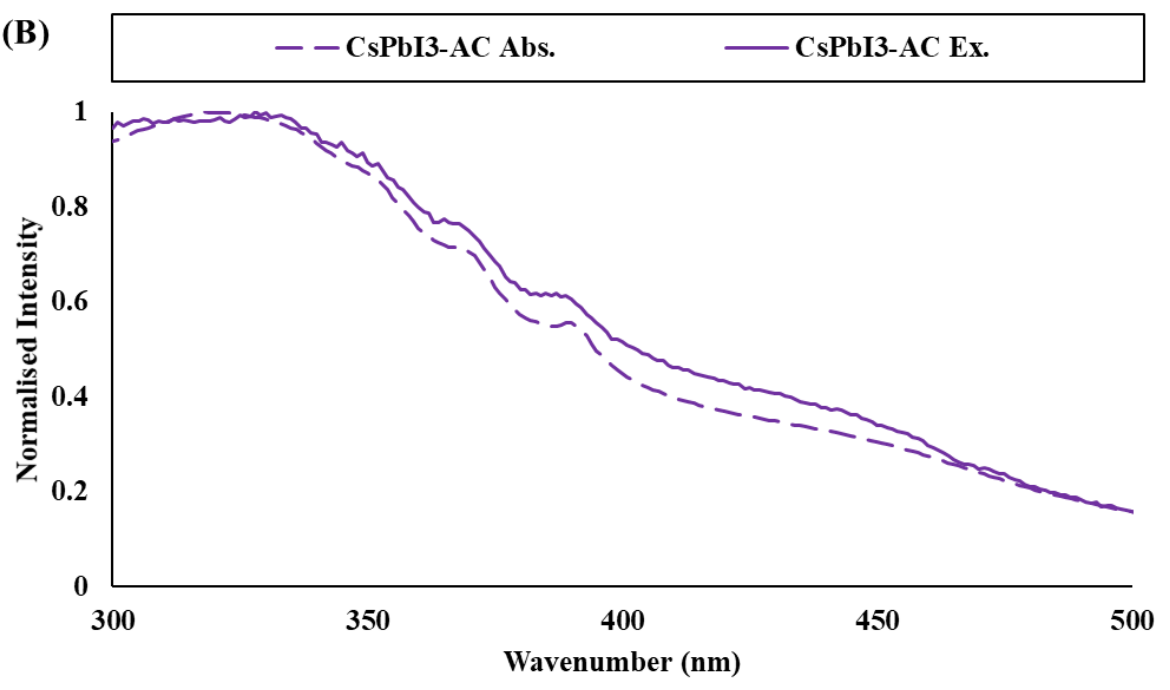

Figure 12w. (A) Comparison between the excitation spectrum of pure $A C$ and $\mathrm{CsPbI}_{3}-\mathrm{AC}$. (B) Comparison between the absorption spectrum and excitation spectrum of $\mathrm{CsPbI}_{3}-\mathrm{AC}$.

From these results, there does appear to be an increase in the electronic excitation of caesium lead halide perovskites due to non-radiative energy transfer from donor anthracene based ligands. 


\section{Photoluminescence Lifetimes of Hot-Injection Synthesised $\mathrm{CsPbX}_{3}$ and $\mathrm{CsPbX}_{3}-\mathrm{AL}$ Nanocrystals}

Conclusive evidence for an energy transfer process occurring between the $\mathrm{AL}$ ligands and $\mathrm{CsPbBr}$ nanocrystals comes from the emission lifetime measurements. From these measurements, it can be seen that the emission lifetime for both $\mathrm{CsPbBr}_{3}-\mathrm{AHpAC}$ and $\mathrm{CsPbBr}_{3}-\mathrm{AC} \mathrm{NCs}$ are greater than that of standard $\mathrm{CsPbBr}_{3}$, a similar result to that seen in the open air synthesised samples (see Figure 12x). Such a result shows that a process that operates on longer time scales to direct excitation of the nanocrystals is inducing $\mathrm{CsPbBr}_{3} \mathrm{NC}$ emission. The best explanation for this is excitation of the anthracene based ligands and subsequent energy transfer into the perovskite.

(A)

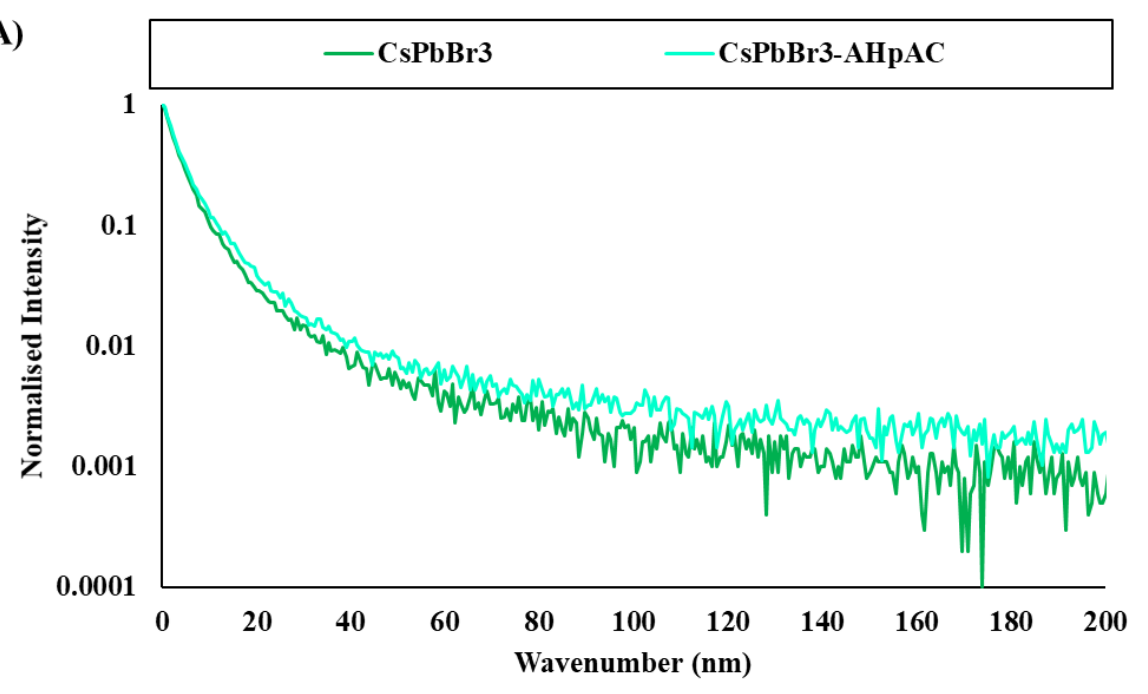

(B)

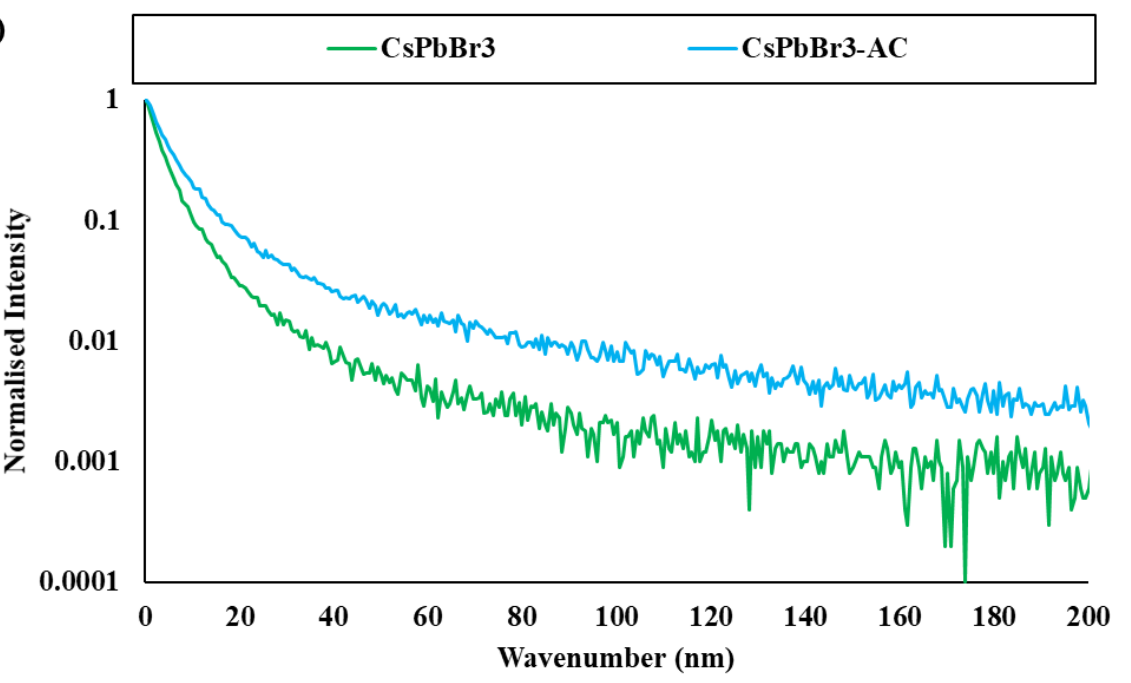

Figure 12x. Comparisons between the emission lifetimes of standard $\mathrm{CsPBr}_{3} \mathrm{NCs}_{\text {to }} \mathrm{CsPbBr}_{3}-$ $A H p A C(A)$ and $\mathrm{CsPbBr}_{3}-\mathrm{AC}(\mathrm{B})$.

$\mathrm{CsPbI}_{3}-\mathrm{AC}$ NCs demonstrates a significantly longer emission lifetime when compared to standard $\mathrm{CsPbI}_{3} \mathrm{NCs}$ (see Figure 12y). Taking the natural log of the data results in a linear graph, allowing for 
the exponential time constant to be calculated from the sample (see Figure 12y). Similar analysis could not be performed for the sample $\mathrm{CsPBr}_{3}-\mathrm{AHpAC}$ due to the non-linearity of the data, meaning that the decay of these samples is multi-exponential and suggestive of an energy cascade system.

(A)

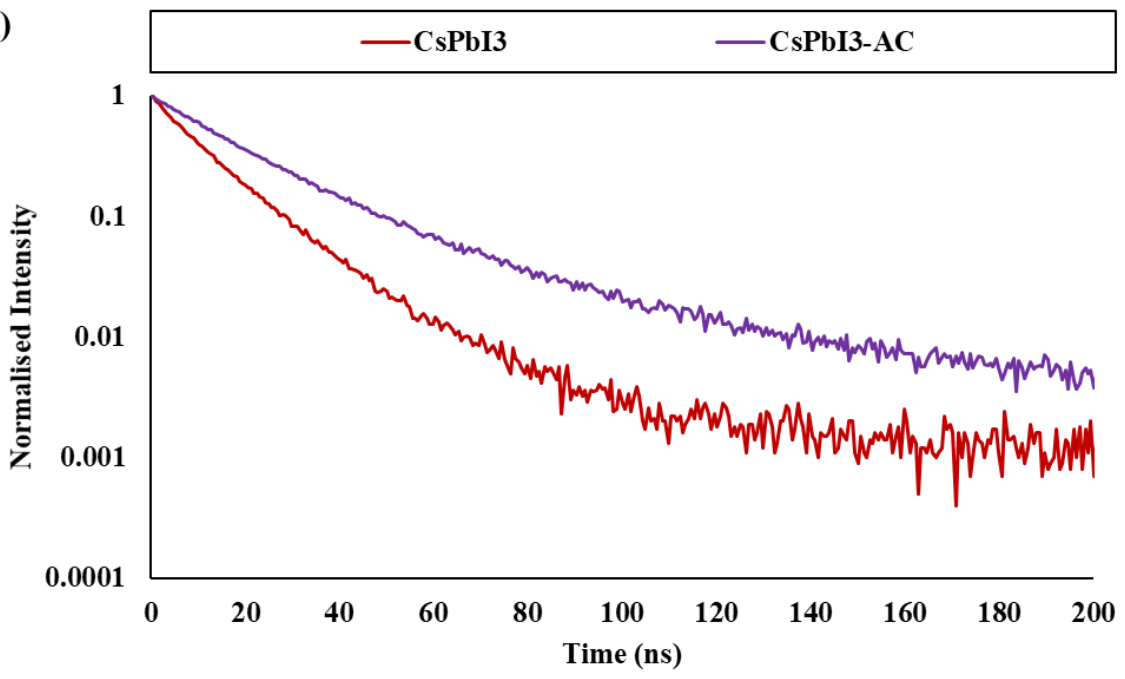

(B)

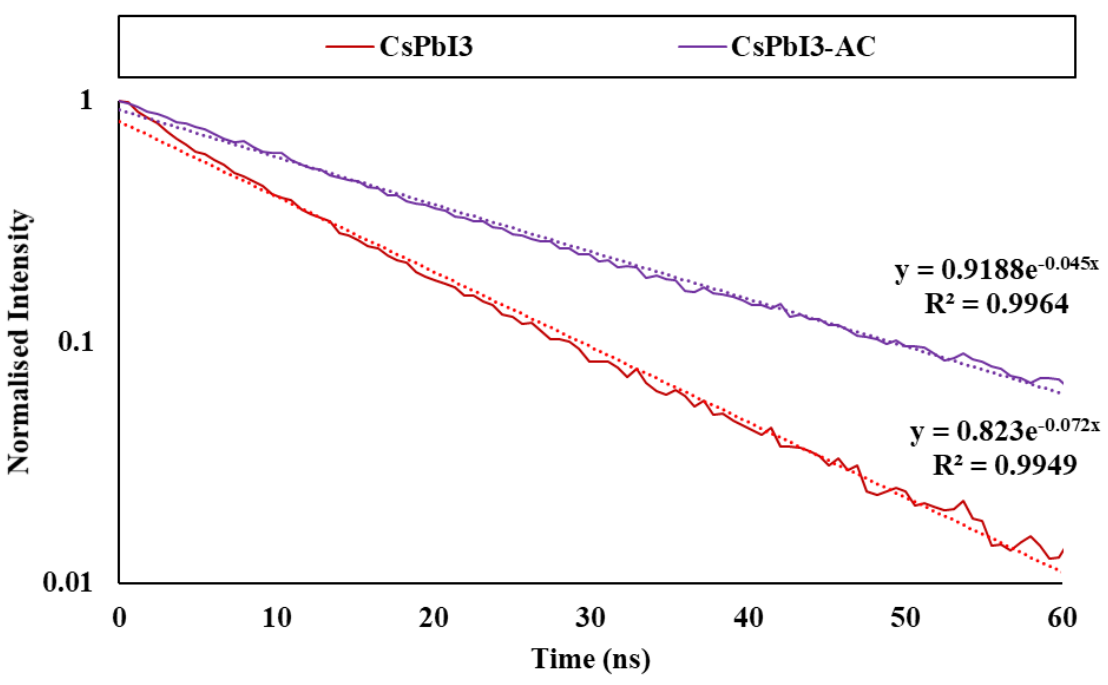

$\tau\left(\mathrm{CsPbI}_{3}\right)=13.89$

$\tau\left(\mathrm{CsPbI}_{3}-\mathrm{AC}\right)=22.22$

Figure 12y. (A) Comparison between the emission lifetimes of standard $\mathrm{CsPbI}_{3} \mathrm{NCs}_{\text {to }} \mathrm{CsPbI}{ }_{3}^{-}$ $A C$. (B) Exponential trendline fits to the experimental data and resulting time constants.

The decay constant for $\mathrm{CsPb}_{3}$ increases by almost $60 \%$ for the standard NCs, strongly suggestive of an energy transfer process between the two chemical species. 


\section{Photoluminescence Quantum Yield of Hot-Injection Synthesised $\mathrm{CsPbX}_{3}$ and $\mathrm{CsPbX}_{3}-\mathrm{AL}$ Nanocrystals}

Using a wavelength of $363 \mathrm{~nm}$ to specifically target the anthracene functional group of $\mathrm{CsPBBr}_{3}-$ $\mathrm{AHpAC}$ and $389 \mathrm{~nm}$ for $\mathrm{CsPbr}_{3}-\mathrm{AC}$ results in a photoluminescence quantum yields (PLQEs) of $58.42 \%$ and $60.60 \%$ respectively. The same measurements but for standard $\mathrm{CsPBr}_{3}$ results in a PLQE of $52.80 \%$.

Standard $\mathrm{CsPbI}_{3} \mathrm{NCs}$ demonstrated PLQEs of $53.66 \%$ when excited by $387 \mathrm{~nm}$ and $55.60 \%$ when excited with $450 \mathrm{~nm}$. $\mathrm{CsPbI}_{3}-\mathrm{AC}$ produced efficiencies of $60.77 \%$ for $387 \mathrm{~nm}$ light and $66.48 \%$ for 450 $\mathrm{nm}$ light. The loss of efficiency when exciting the anthracene based ligands show that the energy transfer process is not $100 \%$. A consequence of this is that the AL negatively effects the PLQE of the material when exciting in the absorption range of the ligand. Such an effect can be even more clearly seen in the best performing $\mathrm{CsPbI}_{3}-\mathrm{AC}$ samples. This sample demonstrated a PLQE of 95.29\% for $450 \mathrm{~nm}$ light, but only $75.46 \%$ for $387 \mathrm{~nm}$ light. It must be noted that this sample was measured after two days of storage. This period of time could have allowed for the loss of AC ligands to the solvent and therefore prevented effective energy transfer.

While AL ligands can prove to be detrimental to the efficiency of $\mathrm{CsPbX}_{3} \mathrm{NCs}$ when illuminated with light that is within the ligand absorption range, all three $\mathrm{CsPbX}_{3}-\mathrm{AL}$ samples demonstrate heightened PLQEs when compared to standard nanocrystals regardless of wavelength. These results suggest that the coordination of anthracene based ligands may act to reduce the severity of efficiency loss mechanisms in caesium lead trihalide perovskite nanocrystals. For perovskite semi-conductors, the major efficiency sink is due to crystallographic defects that facilitate trap-assisted recombination. Many of these crystallographic defects are located at the surface of the nanocrystal. Ligands can passivate these surface defects and improve the efficiency of the material. The increase in PLQE in samples possessing ALs suggests that these ligands are more effective at neutralising surface defects, reducing efficiency loss and thereby increasing performance.

To circumvent the decrease in efficiency for wavelengths within the absorption range of the AL ligands, the photon multiplication process of singlet fission needs to be efficiently utilised. The only way to do this would be to synthesis $\mathrm{CsPbI}_{3}$ nanocrystals that possess a band gap of at least $1.77 \mathrm{eV}$ Bae et al. ${ }^{62}$ Such a band gap would correspond to a peak emission wavelength of at least $700 \mathrm{~nm}$. While such a wavelength is possible for $\mathrm{CsPbI}_{3}$ nanocrystals of a sufficient size, such emission was not achieved in this project. 


\section{2c. Concluding Remarks on Hot-Injection Synthesised $\mathrm{CsPbX}_{3}$ and $\mathrm{CsPbX}_{3}-\mathrm{AL}$ Nanocrystals}

Due to time constraints, TEM images of the $\mathrm{CsPbX}_{3}-\mathrm{AL}$ nanocrystals could not be performed, in addition, the non-washed particles would have been difficult at best to observe due to the presence of organic material potentially coating and obscuring the nanocrystals to the electron beam. However, based upon the collected photoluminescence data, non-radiative energy transfer between the anthracene functional groups and caesium lead tribromide/triiodide does occur.

Though high concentrations of $N$-(7-aminoheptyl)-anthracene-9-carboxamide (AHpAC) and anthracene-9-carboxylic acid (AC) prove to be detrimental to perovskite nanocrystal formation, low concentrations of these ligands allow for the formation of nanoparticles with comparable photoluminescence properties and improved quantum yields, indicating that the anthracene based ligands have a positive influence on the nanocrystal quality.

The samples demonstrated redshifted anthracene absorption and excitation, the latter of which is shown to result in perovskite emission for both $\mathrm{CsPBr}_{3}$ and $\mathrm{CsPbI}_{3}$ nanocrystals. Additionally, the lack of anthracene emission means that a competitive process to anthracene fluorescence must be occurring on a significant scale, suggestive of an energy transfer process and close proximity of the anthracene functional groups to the nanocrystals which further indicates successful coordination. Emission lifetime measurements support this conclusion as the anthracene possessing samples demonstrate a lengthened emission decay which correlates well with a scenario in which the ligand is excited, and this absorbed energy is then transferred to the perovskite.

While singlet fission was not observed to be taking place, this research has proven that caesium lead trihalide nanocrystals can be synthesised with anthracene based ligands. The lack of singlet fission can be accounted for by the unideal perovskite band gap energies for triplet energy harvesting and the presence of air within the samples. Follow up research would need to synthesis $\mathrm{CsPbI}_{3}-\mathrm{AC}$ nanocrystals, or similar anthracene coordinated lead halide perovskites, that emit at wavelengths longer than $700 \mathrm{~nm}$. Comparisons between different PLQE measurements that have specifically targeted the ligand excitation range and perovskite excitation range would be sufficient to indicate a photon multiplication process. This is a relatively simple endeavour and should be worked towards because, if achieved, the coordination of anthracene based ligands to perovskite nanocrystals, which currently can display PLQEs of over $90 \%$ at their best, could reached quantum efficiencies of over $100 \%$. 


\section{Concluding Remarks on the Functionalisation of Lead Halide Perovskites with Anthracene}

Lead halide perovskites have the potential to become the basis of the next generation of photovoltaic devices due to their ease of synthesis and exceptional optoelectronic properties. However, as with all semiconducting technologies, perovskite based solar cells are severely limited in terms of their maximum theoretical efficiency. To overcome this theoretical maximum, studies have sought to couple organic fluorophores to inorganic semiconducting nanomaterials and utilise specific energy transfer processes to either increase the range of wavelengths that can be utilised or decrease thermalisation losses. Anthracene is an acene molecule that is capable of endothermic singlet fission, a process that is capable of generating two triplet states from a single excitation event. Coupling such a molecule to an efficient semiconducting material, such as a lead halide perovskite could theoretically result in a highly efficient light harvesting material. The aim of this study was to functionalise lead halide perovskites with anthracene functional groups and to observe if any form of energy transfer, with specific focus on singlet fission, was occurring between the organic fluorophore and the inorganic semiconductor.

Three different strategies were utilised in the attempt to achieve this goal. Two of these strategies aimed to functionalise the organic spacer cations of 2D Ruddlesden-Popper perovskites with anthracene. The first method involved the slow vaporisation of a solution containing a lead halide and an (anthracene-9-carboxamide)alkylaminium halide salt (ACAAX) to induce the crystallisation of a two-dimensional perovskite thin-film. While X-Ray Diffraction data could not be collected, which would have provided conclusive evidence on whether or not the method was successful, absorption and photoluminescent data strongly suggest that a perovskite phase was not be formed. However, this does not prove that the anthracene functional group cannot be incorporated into the crystal structure of two-dimensional perovskites as only one method of thin-film synthesis was employed. Alternative methods of perovskite thin-film synthesis should be investigated as these experiments did provide promising results. Namely, the incorporation of lead halides does not prevent excimer formation, meaning that the anthracene functional groups are still capable of interacting with each other in the electronic excited state. Such interactions are crucial for singlet fission and therefore a successfully synthesised anthracene functionalised two-dimensional perovskite may be capable of performing singlet fission. However, to utilise singlet fission requires tuning the band gap of the perovskite to be comparable to the energy of triplet excited state of anthracene. This will inevitably require the synthesis of a $2 \mathrm{D} / 3 \mathrm{D}$ hybrid perovskite.

The second method sought to convert lead halide nanocrystals into 2D Ruddlesden-Popper perovskite nanocrystals by exposing them to a solution of ACAAX. Similarly, to the previously discussed method, the two precursors did not form a perovskite. Instead of the molecule intercalating into the 
nanoparticles particles and converting them into a perovskite, the ACAAX salt facilitated the aggregation and even degradation of the nanocrystals. However, while the thin-films demonstrated significant excimer emission, the solution based samples demonstrated significant scattering of light sources, and what photoluminescence that was observed was predominantly monomer emission. Therefore, the intercalation method of 2D Ruddlesden-Popper perovskite nanocrystal formation is not an ideal synthesis method.

The third method of functionalising lead halide perovskites sought to stabilise caesium lead trihalide nanocrystals $\left(\mathrm{CsPbX}_{3} \mathrm{NCs}\right)$ with anthracene functionalised ligands. Two different synthetic methods were utilised, however, while both provided positive results, one methodology was significantly more reliable than the other and produced the best results. Initial experiments sought to substitute the more traditionally utilised oleic acid (OA) and oleylamine (OAm) ligands with anthracene-9-carboxylic acid (AC) and $N$-(7-aminoheptyl)anthracene-9-carboxamide (AHpAC). These experiments showed that the complete removal and substitution of either OA or OAm resulted in the unsuccessful stabilisation of the perovskite phase. Based upon these results, further attempts sought to add either $\mathrm{AC}$ or $\mathrm{AHpAC}$ as additional reagents to the synthesis of $\mathrm{CsPbX}_{3} \mathrm{NCs}$. While the mere presence of significant quantities of either anthracene derivative is also detrimental to nanocrystal stabilisation, reducing the quantity allows for the successful stabilisation of $\mathrm{CsPbX}_{3} \mathrm{NCs}$. Analysing the absorption spectrum of these samples showed the presence of the characteristic absorption peaks of anthracene. Analysis of the photoluminescent properties of the NC solutions showed that the excitation of anthracene was contributing to the emission of the perovskite nanocrystals.

While an increased PLQE was not observed, this does not mean that singlet fission cannot boost the performance of a lead halide perovskite. Firstly, the emission of the perovskites indicated band gaps that were not appropriately matched to the energy of the triplet excited state of the anthracene ligands. Secondly, the samples were exposed to oxygen which is a factor that is detrimental to the process of singlet fission. To build upon this work, the next step is to synthesis a sample of anthracene functionalised lead halide perovskite nanocrystals with a band gap that is equal or smaller than the energy of the triplet excited state of anthracene. Such a sample would provide the best conditions for the Dexter Energy Transfer of singlet fission generated triplet excited states.

Therefore, as caesium lead tribromide and triiodide nanocrystals were functionalised with anthracene ligands and energy transfer between the ligands and semiconductors was observed, the ultimate aim of this research was achieved. 


\section{References}

1. Kopp, G.; Lawrence, G.; Rottman, G., The Total Irradiance Monitor (TIM): Science Results. Solar Physics 2005, 230 (1), 129-139.

2. $\quad$ Kopp, G.; Lean, J. L., A new, lower value of total solar irradiance: Evidence and climate significance. Geophys. Res. Lett. 2011, 38 (1).

3. Richter, A.; Hermle, M.; Glunz, S. W., Reassessment of the Limiting Efficiency for Crystalline Silicon Solar Cells. IEEE Journal of Photovoltaics 2013, 3 (4), 1184-1191.

4. $\quad$ Enrichi, F.; Righini, G., Solar Cells and Light Management: Materials, Strategies and Sustainability. Elsevier Science: 2019.

5. Green, M. A.; Hishikawa, Y.; Dunlop, E. D.; Levi, D. H.; Hohl-Ebinger, J.; Ho-Baillie, A. W. Y., Solar cell efficiency tables (version 51). Progress in Photovoltaics: Research and Applications 2018, 26 (1), 3-12.

6. Tiedje, T.; Yablonovitch, E.; Cody, G. D.; Brooks, B. G., Limiting efficiency of silicon solar cells. IEEE Transactions on Electron Devices 1984, 31 (5), 711-716.

7. Yoshikawa, K.; Kawasaki, H.; Yoshida, W.; Irie, T.; Konishi, K.; Nakano, K.; Uto, T.; Adachi, D.; Kanematsu, M.; Uzu, H.; Yamamoto, K., Silicon heterojunction solar cell with interdigitated back contacts for a photoconversion efficiency over 26\%. Nature Energy 2017, 2 (5), 17032.

8. MØLler, C. K., Crystal Structure and Photoconductivity of Cæsium Plumbohalides. Nature 1958, 182 (4647), 1436-1436.

9. Kojima, A.; Teshima, K.; Shirai, Y.; Miyasaka, T., Organometal Halide Perovskites as Visible-Light Sensitizers for Photovoltaic Cells. J. Am. Chem. Soc. 2009, 131 (17), 6050-6051. 10. Im, J.-H.; Lee, C.-R.; Lee, J.-W.; Park, S.-W.; Park, N.-G., 6.5\% efficient perovskite quantum-dot-sensitized solar cell. Nanoscale 2011, 3 (10), 4088-4093.

11. Caputo, M.; Cefarin, N.; Radivo, A.; Demitri, N.; Gigli, L.; Plaisier, J. R.; Panighel, M.; Di Santo, G.; Moretti, S.; Giglia, A.; Polentarutti, M.; De Angelis, F.; Mosconi, E.; Umari, P.; Tormen, M.; Goldoni, A., Electronic structure of MAPbI3 and MAPbCl3: importance of band alignment. Sci. Rep. 2019, 9 (1), 15159.

12. Leguy, A. M. A.; Azarhoosh, P.; Alonso, M. I.; Campoy-Quiles, M.; Weber, O. J.; Yao, J.; Bryant, D.; Weller, M. T.; Nelson, J.; Walsh, A.; van Schilfgaarde, M.; Barnes, P. R. F., Experimental and theoretical optical properties of methylammonium lead halide perovskites. Nanoscale 2016, 8 (12), 6317-6327.

13. Papavassiliou, G. C.; Koutselas, I. B.; Terzis, A.; Whangbo, M. H., Structural and electronic properties of the natural quantum-well system (C6H5CH2CH2NH3)2SnI4. Solid State Communications 1994, 91 (9), 695-698.

14. Katan, C.; Pedesseau, L.; Kepenekian, M.; Rolland, A.; Even, J., Interplay of spin-orbit coupling and lattice distortion in metal substituted 3D tri-chloride hybrid perovskites. Journal of Materials Chemistry A 2015, 3 (17), 9232-9240.

15. Lee, M. M.; Teuscher, J.; Miyasaka, T.; Murakami, T. N.; Snaith, H. J., Efficient Hybrid Solar Cells Based on Meso-Superstructured Organometal Halide Perovskites. Science 2012, 338 (6107), 643-647.

16. Bisquert, J., Chemical capacitance of nanostructured semiconductors: its origin and significance for nanocomposite solar cells. Phys. Chem. Chem. Phys. 2003, 5 (24), 5360-5364.

17. Noh, J. H.; Im, S. H.; Heo, J. H.; Mandal, T. N.; Seok, S. I., Chemical Management for Colorful, Efficient, and Stable Inorganic-Organic Hybrid Nanostructured Solar Cells. Nano Letters 2013, 13 (4), 1764-1769.

18. Zhou, H.; Chen, Q.; Li, G.; Luo, S.; Song, T.-b.; Duan, H.-S.; Hong, Z.; You, J.; Liu, Y.; Yang, Y., Interface engineering of highly efficient perovskite solar cells. Science 2014, 345 (6196), 542.

19. Eperon, G. E.; Stranks, S. D.; Menelaou, C.; Johnston, M. B.; Herz, L. M.; Snaith, H. J., Formamidinium lead trihalide: a broadly tunable perovskite for efficient planar heterojunction solar cells. Energy Environ. Sci. 2014, 7 (3), 982-988. 
20. Lee, J. W.; Seol, D. J.; Cho, A. N.; Park, N. G., High-Efficiency Perovskite Solar Cells Based on the Black Polymorph of HC(NH2)2PbI3. Advanced Materials 2014, 26 (29), 4991-4998.

21. Yang, W. S.; Noh, J. H.; Jeon, N. J.; Kim, Y. C.; Ryu, S.; Seo, J.; Seok, S. I., Highperformance photovoltaic perovskite layers fabricated through intramolecular exchange. Science 2015, 348 (6240), 1234.

22. Jeon, N. J.; Noh, J. H.; Kim, Y. C.; Yang, W. S.; Ryu, S.; Seok, S. I., Solvent engineering for high-performance inorganic-organic hybrid perovskite solar cells. Nature Materials 2014, 13 (9), 897-903.

23. Jeon, N. J.; Noh, J. H.; Yang, W. S.; Kim, Y. C.; Ryu, S.; Seo, J.; Seok, S. I., Compositional engineering of perovskite materials for high-performance solar cells. Nature 2015, 517 (7535), 476480 .

24. Cho, K. T.; Paek, S.; Grancini, G.; Roldán-Carmona, C.; Gao, P.; Lee, Y.; Nazeeruddin, M. K., Highly efficient perovskite solar cells with a compositionally engineered perovskite/hole transporting material interface. Energy Environ. Sci. 2017, 10 (2), 621-627.

25. Yang, W. S.; Park, B.-W.; Jung, E. H.; Jeon, N. J.; Kim, Y. C.; Lee, D. U.; Shin, S. S.; Seo, J.; Kim, E. K.; Noh, J. H.; Seok, S. I., Iodide management in formamidinium-lead-halide-based perovskite layers for efficient solar cells. Science 2017, 356 (6345), 1376-1379.

26. Saliba, M.; Matsui, T.; Seo, J.-Y.; Domanski, K.; Correa-Baena, J.-P.; Nazeeruddin, M. K.; Zakeeruddin, S. M.; Tress, W.; Abate, A.; Hagfeldt, A.; Grätzel, M., Cesium-containing triple cation perovskite solar cells: improved stability, reproducibility and high efficiency. Energy Environ. Sci. 2016, 9 (6), 1989-1997.

27. Choi, H.; Jeong, J.; Kim, H.-B.; Kim, S.; Walker, B.; Kim, G.-H.; Kim, J. Y., Cesium-doped methylammonium lead iodide perovskite light absorber for hybrid solar cells. Nano Energy 2014, 7 , $80-85$.

28. Lee, J.-W.; Kim, D.-H.; Kim, H.-S.; Seo, S.-W.; Cho, S. M.; Park, N.-G., Formamidinium and Cesium Hybridization for Photo- and Moisture-Stable Perovskite Solar Cell. Advanced Energy Materials 2015, 5 (20), 1501310.

29. McMeekin, D. P.; Sadoughi, G.; Rehman, W.; Eperon, G. E.; Saliba, M.; Hörantner, M. T.; Haghighirad, A.; Sakai, N.; Korte, L.; Rech, B.; Johnston, M. B.; Herz, L. M.; Snaith, H. J., A mixedcation lead mixed-halide perovskite absorber for tandem solar cells. Science 2016, 351 (6269), 151.

30. Kulbak, M.; Cahen, D.; Hodes, G., How Important Is the Organic Part of Lead Halide Perovskite Photovoltaic Cells? Efficient CsPbBr3 Cells. The Journal of Physical Chemistry Letters 2015, 6 (13), 2452-2456.

31. Protesescu, L.; Yakunin, S.; Bodnarchuk, M. I.; Krieg, F.; Caputo, R.; Hendon, C. H.; Yang, R. X.; Walsh, A.; Kovalenko, M. V., Nanocrystals of Cesium Lead Halide Perovskites (CsPbX3, X = $\mathrm{Cl}, \mathrm{Br}$, and I): Novel Optoelectronic Materials Showing Bright Emission with Wide Color Gamut. Nano Letters 2015, 15 (6), 3692-3696.

32. Kulbak, M.; Gupta, S.; Kedem, N.; Levine, I.; Bendikov, T.; Hodes, G.; Cahen, D., Cesium Enhances Long-Term Stability of Lead Bromide Perovskite-Based Solar Cells. The Journal of Physical Chemistry Letters 2016, 7 (1), 167-172.

33. Luo, P.; Xia, W.; Zhou, S.; Sun, L.; Cheng, J.; Xu, C.; Lu, Y., Solvent Engineering for Ambient-Air-Processed, Phase-Stable CsPbI3 in Perovskite Solar Cells. The Journal of Physical Chemistry Letters 2016, 7 (18), 3603-3608.

34. Eperon, G. E.; Paternò, G. M.; Sutton, R. J.; Zampetti, A.; Haghighirad, A. A.; Cacialli, F.; Snaith, H. J., Inorganic caesium lead iodide perovskite solar cells. Journal of Materials Chemistry A 2015, 3 (39), 19688-19695.

35. Swarnkar, A.; Marshall, A. R.; Sanehira, E. M.; Chernomordik, B. D.; Moore, D. T.; Christians, J. A.; Chakrabarti, T.; Luther, J. M., Quantum dot-induced phase stabilization of $\alpha$ $\mathrm{CsPbI}<\mathrm{sub}>3</$ sub $>$ perovskite for high-efficiency photovoltaics. Science 2016, 354 (6308), 92-95. 36. Zhao, Q.; Hazarika, A.; Schelhas, L. T.; Liu, J.; Gaulding, E. A.; Li, G.; Zhang, M.; Toney, M. F.; Sercel, P. C.; Luther, J. M., Size-Dependent Lattice Structure and Confinement Properties in CsPbI3 Perovskite Nanocrystals: Negative Surface Energy for Stabilization. ACS Energy Letters 2020, 5 (1), 238-247. 
37. Wang, K.; Jin, Z.; Liang, L.; Bian, H.; Bai, D.; Wang, H.; Zhang, J.; Wang, Q.; Liu, S., Allinorganic cesium lead iodide perovskite solar cells with stabilized efficiency beyond $15 \%$. Nature Communications 2018, 9 (1), 4544.

38. Wang, J.; Zhang, J.; Zhou, Y.; Liu, H.; Xue, Q.; Li, X.; Chueh, C.-C.; Yip, H.-L.; Zhu, Z.; Jen, A. K. Y., Highly efficient all-inorganic perovskite solar cells with suppressed non-radiative recombination by a Lewis base. Nature Communications 2020, 11 (1), 177.

39. Ruf, F.; Magin, A.; Schultes, M.; Ahlswede, E.; Kalt, H.; Hetterich, M., Excitonic nature of optical transitions in electroabsorption spectra of perovskite solar cells. Applied Physics Letters 2018, $112(8), 083902$.

40. Hawash, Z.; Ono, L. K.; Qi, Y., Recent Advances in Spiro-MeOTAD Hole Transport Material and Its Applications in Organic-Inorganic Halide Perovskite Solar Cells. Advanced Materials Interfaces 2018, 5 (1), 1700623.

41. D'Innocenzo, V.; Grancini, G.; Alcocer, M. J. P.; Kandada, A. R. S.; Stranks, S. D.; Lee, M. M.; Lanzani, G.; Snaith, H. J.; Petrozza, A., Excitons versus free charges in organo-lead tri-halide perovskites. Nature Communications 2014, 5, 3586.

42. $\quad$ Steirer, K. X.; Schulz, P.; Teeter, G.; Stevanovic, V.; Yang, M.; Zhu, K.; Berry, J. J., Defect Tolerance in Methylammonium Lead Triiodide Perovskite. ACS Energy Letters 2016, 1 (2), 360-366. 43. Yin, W.-J.; Shi, T.; Yan, Y., Unusual defect physics in CH3NH3PbI3 perovskite solar cell absorber. Applied Physics Letters 2014, 104 (6), 063903.

44. Wu, X.; Trinh, M. T.; Niesner, D.; Zhu, H.; Norman, Z.; Owen, J. S.; Yaffe, O.; Kudisch, B. J.; Zhu, X. Y., Trap States in Lead Iodide Perovskites. J. Am. Chem. Soc. 2015, 137 (5), 2089-2096. 45. Wehrenfennig, C.; Eperon, G. E.; Johnston, M. B.; Snaith, H. J.; Herz, L. M., High Charge Carrier Mobilities and Lifetimes in Organolead Trihalide Perovskites. Advanced Materials 2014, 26 (10), 1584-1589.

46. Davies, C. L.; Filip, M. R.; Patel, J. B.; Crothers, T. W.; Verdi, C.; Wright, A. D.; Milot, R. L.; Giustino, F.; Johnston, M. B.; Herz, L. M., Bimolecular recombination in methylammonium lead triiodide perovskite is an inverse absorption process. Nature Communications 2018, 9 (1), 293.

47. Frost, J. M.; Butler, K. T.; Brivio, F.; Hendon, C. H.; van Schilfgaarde, M.; Walsh, A., Atomistic Origins of High-Performance in Hybrid Halide Perovskite Solar Cells. Nano Letters 2014, 14 (5), 2584-2590.

48. Vincent, B. R.; Robertson, K. N.; Cameron, T. S.; Knop, O., Alkylammonium lead halides. Part 1. Isolated PbI64- ions in (CH3NH3)4PbI6•2H2O. Can. J. Chem. 1987, 65 (5), 1042-1046. 49. Leguy, A. M. A.; Hu, Y.; Campoy-Quiles, M.; Alonso, M. I.; Weber, O. J.; Azarhoosh, P.; van Schilfgaarde, M.; Weller, M. T.; Bein, T.; Nelson, J.; Docampo, P.; Barnes, P. R. F., Reversible Hydration of CH3NH3PbI3 in Films, Single Crystals, and Solar Cells. Chem. Mater. 2015, 27 (9), 3397-3407.

50. $\quad$ Smith, I. C.; Hoke, E. T.; Solis-Ibarra, D.; McGehee, M. D.; Karunadasa, H. I., A Layered Hybrid Perovskite Solar-Cell Absorber with Enhanced Moisture Stability. Angewandte Chemie International Edition 2014, 53 (42), 11232-11235.

51. Tsai, H.; Nie, W.; Blancon, J.-C.; Stoumpos, C. C.; Asadpour, R.; Harutyunyan, B.; Neukirch, A. J.; Verduzco, R.; Crochet, J. J.; Tretiak, S.; Pedesseau, L.; Even, J.; Alam, M. A.; Gupta, G.; Lou, J.; Ajayan, P. M.; Bedzyk, M. J.; Kanatzidis, M. G.; Mohite, A. D., High-efficiency two-dimensional Ruddlesden-Popper perovskite solar cells. Nature 2016, 536, 312.

52. Ren, H.; Yu, S.; Chao, L.; Xia, Y.; Sun, Y.; Zuo, S.; Li, F.; Niu, T.; Yang, Y.; Ju, H.; Li, B.; Du, H.; Gao, X.; Zhang, J.; Wang, J.; Zhang, L.; Chen, Y.; Huang, W., Efficient and stable Ruddlesden-Popper perovskite solar cell with tailored interlayer molecular interaction. Nature Photonics 2020.

53. Yaffe, O.; Chernikov, A.; Norman, Z. M.; Zhong, Y.; Velauthapillai, A.; van der Zande, A.; Owen, J. S.; Heinz, T. F., Excitons in ultrathin organic-inorganic perovskite crystals. Physical Review $B$ 2015, 92 (4), 045414.

54. Xing, G.; Wu, B.; Wu, X.; Li, M.; Du, B.; Wei, Q.; Guo, J.; Yeow, E. K. L.; Sum, T. C.; Huang, W., Transcending the slow bimolecular recombination in lead-halide perovskites for electroluminescence. Nature Communications 2017, 8 (1), 14558. 
55. Koutselas, I. B.; Ducasse, L.; Papavassiliou, G. C., Electronic properties of three- and lowdimensional semiconducting materials with $\mathrm{Pb}$ halide and $\mathrm{Sn}$ halide units. Journal of Physics: Condensed Matter 1996, 8 (32), 5953-5953.

56. Huang, Z.; Li, X.; Mahboub, M.; Hanson, K. M.; Nichols, V. M.; Le, H.; Tang, M. L.; Bardeen, C. J., Hybrid Molecule-Nanocrystal Photon Upconversion Across the Visible and NearInfrared. Nano Letters 2015, 15 (8), 5552-5557.

57. Mongin, C.; Garakyaraghi, S.; Razgoniaeva, N.; Zamkov, M.; Castellano, F. N., Direct observation of triplet energy transfer from semiconductor nanocrystals. Science 2016, 351 (6271), 369-372.

58. Ehrler, B.; Wilson, M. W. B.; Rao, A.; Friend, R. H.; Greenham, N. C., Singlet Exciton Fission-Sensitized Infrared Quantum Dot Solar Cells. Nano Letters 2012, 12 (2), 1053-1057.

59. Davis, N. J. L. K.; Allardice, J. R.; Xiao, J.; Petty, A. J.; Greenham, N. C.; Anthony, J. E.; Rao, A., Singlet Fission and Triplet Transfer to PbS Quantum Dots in TIPS-Tetracene Carboxylic Acid Ligands. The Journal of Physical Chemistry Letters 2018, 9 (6), 1454-1460.

60. Vaubel, G.; Baessler, H., Determination of the band-gap in anthracene. Physics Letters A 1968, 27 (6), 328-329.

61. Bae, Y. J.; Christensen, J.; Kang, G.; Malliakas, C.; Zhou, J.; Nelson, J.; Young, R.; Wu, Y.L.; Van Duyne, R.; Schatz, G.; Wasielewski, M., Design principles for efficient singlet fission in anthracene-based organic semiconductors. SPIE: 2019; Vol. 11084.

62. Bae, Y. J.; Kang, G.; Malliakas, C. D.; Nelson, J. N.; Zhou, J.; Young, R. M.; Wu, Y.-L.; Van Duyne, R. P.; Schatz, G. C.; Wasielewski, M. R., Singlet Fission in 9,10-

Bis(phenylethynyl)anthracene Thin Films. J. Am. Chem. Soc. 2018, 140 (45), 15140-15144.

63. Bouas-Laurent, H.; Castellan, A.; Desvergne, J.-P.; Lapouyade, R., Photodimerization of anthracenes in fluid solutions: (part 2) mechanistic aspects of the photocycloaddition and of the photochemical and thermal cleavage. Chem. Soc. Rev. 2001, 30 (4), 248-263.

64. Raga, S. R.; Jung, M.-C.; Lee, M. V.; Leyden, M. R.; Kato, Y.; Qi, Y., Influence of Air Annealing on High Efficiency Planar Structure Perovskite Solar Cells. Chem. Mater. 2015, 27 (5), 1597-1603.

65. Takeoka, Y.; Asai, K.; Rikukawa, M.; Sanui, K., Systematic Studies on Chain Lengths, Halide Species, and Well Thicknesses for Lead Halide Layered Perovskite Thin Films. Bull. Chem. Soc. Jpn. 2006, 79 (10), 1607-1613.

66. Cheng, Z.; Lin, J., Layered organic-inorganic hybrid perovskites: structure, optical properties, film preparation, patterning and templating engineering. CrystEngComm 2010, 12 (10), 2646-2662.

67. Dohner, E. R.; Hoke, E. T.; Karunadasa, H. I., Self-Assembly of Broadband White-Light Emitters. J. Am. Chem. Soc. 2014, 136 (5), 1718-1721.

68. Oswald, I. W. H.; Koegel, A. A.; Neilson, J. R., General Synthesis Principles for RuddlesdenPopper Hybrid Perovskite Halides from a Dynamic Equilibrium. Chem. Mater. 2018, 30 (23), 86068614.

69. Hassan, Y.; Song, Y.; Pensack, R. D.; Abdelrahman, A. I.; Kobayashi, Y.; Winnik, M. A.; Scholes, G. D., Structure-Tuned Lead Halide Perovskite Nanocrystals. Advanced Materials 2016, 28 (3), 566-573.

70. Gorman, J.; Pandya, R.; Allardice, J. R.; Price, M. B.; Schmidt, T. W.; Friend, R. H.; Rao, A.; Davis, N. J. L. K., Excimer Formation in Carboxylic Acid-Functionalized Perylene Diimides Attached to Silicon Dioxide Nanoparticles. The Journal of Physical Chemistry C 2019, 123 (6), 3433 3440 .

71. Zhang, Y.; Saidaminov, M. I.; Dursun, I.; Yang, H.; Murali, B.; Alarousu, E.; Yengel, E.; Alshankiti, B. A.; Bakr, O. M.; Mohammed, O. F., Zero-Dimensional Cs4PbBr6 Perovskite Nanocrystals. The Journal of Physical Chemistry Letters 2017, 8 (5), 961-965.

72. Akkerman, Q. A.; Park, S.; Radicchi, E.; Nunzi, F.; Mosconi, E.; De Angelis, F.; Brescia, R.; Rastogi, P.; Prato, M.; Manna, L., Nearly Monodisperse Insulator Cs4PbX6 (X = Cl, Br, I) Nanocrystals, Their Mixed Halide Compositions, and Their Transformation into CsPbX3 Nanocrystals. Nano Letters 2017, 17 (3), 1924-1930.

73. Xie, Q.; Wu, D.; Wang, X.; Li, Y.; Fang, F.; Wang, Z.; Ma, Y.; Su, M.; Peng, S.; Liu, H.; Wang, K.; Sun, X. W., Branched capping ligands improve the stability of cesium lead halide (CsPbBr3) perovskite quantum dots. Journal of Materials Chemistry C 2019, 7 (36), 11251-11257. 
